SEISMIC PERFORMANCE OF SYMMETRIC STEEL MOMENT FRAMES WITH RANDOM REACTIVE WEIGHT DISTRIBUTIONS

\author{
A Thesis \\ presented to \\ the Faculty of California Polytechnic State University, \\ San Luis Obispo
}

In Partial Fulfillment

of the Requirements for the Degree

Master of Science in Civil and Environmental Engineering

by

Conner Francis Frederick Williamson

December 2012 
(C) 2012

Conner Francis Frederick Williamson ALL RIGHTS RESERVED 
COMMITTEE MEMBERSHIP

TITLE:

Seismic Performance of Symmetric Steel Moment

Frames with Random Reactive Weight Distributions

AUTHOR:

Conner Francis Frederick Williamson

DATE SUBMITTED:

December 2012

COMMITTEE CHAIR:

Bing Qu, Assistant Professor

COMMITEE MEMBER: Charles Chadwell, Associate Professor

COMMITTEE MEMBER: Daniel Jansen, Professor 


\section{ABSTRACT \\ Seismic Performance of Symmetric Steel Moment Frames with Random Reactive Weight Distributions}

\section{Conner Francis Frederick Williamson}

When a structure undergoes seismic excitation, the intensities and spatial distributions of the reactive weights on the structure may not be the same as those assumed in original design. Such a difference is inevitable due to many facts with the random nature (e.g., redistribution of live load), resulting in accidental eccentricity and consequently torsional response in the system. The added torsion can cause excessive deformation and premature failure of the lateral force resisting system. Its detrimental effect is typically accounted for in most building design codes with an arbitrarily specified accidental eccentricity value. While it tends to amplify drift response of buildings under earthquake excitations, it is unclear whether the code specified accidental eccentricity is quantitatively adequate or not in seismic fragility assessment of steel moment frames (including low-rise, mid-rise and high-rise frames) with random reactive weight distributions. This thesis applies surveyed dead and live load intensities and distributions to three representative steel moment resisting frame structures that have been widely investigated in a series of projects under the collaboration of the Structural Engineers Association of California (SEAOC), the Applied Technology Council (ATC), and Consortium of Universities for Research in Earthquake Engineering (CUREE), known as SAC. Based on an extensive parametric study and incremental nonlinear dynamic analyses, it is found that variable load intensity and eccentricity had negligible impacts on the inter-story drifts of the low- and high-rise steel moment frames. However, they affect to a higher degree the performance of the mid-rise steel moment frames. Moreover, it is found that under the maximum considered earthquake (MCE) event, the actual drifts in steel moment frames with random reactive weight distributions can be conservatively captured through consideration of the code specified accidental eccentricities.

Keywords: Torsion, accidental eccentricity, fragility analysis 


\section{ACKNOWLEDGMENTS}

I would like to express my gratitude for Prof. Bing Qu. Without his passion for the material, I may not have pursued a thesis project, and without his guidance, I probably would not have finished this thesis project.

I would like to thank Prof. Charles Chadwell and Prof. Daniel Jansen for taking the time to serve on my thesis defense committee.

I am very appreciative of the financial support from the National Science Foundation and the Cal Poly Extramural Funding Initiative Program.

I would also like to thank Ron Leverett, Civil and Environmental Engineering Computer Technician, his assistance in the computer lab was invaluable. Without his help, the necessary computer simulations would not have been able to be completed in such a timely, and reasonably effortless, manner.

Finally, I am grateful that my parents have always provided me with the opportunities to pursue my interests, and my brother, Colin, for finding the things that spell check could not. 


\section{TABLE OF CONTENTS}

LIST OF TABLES .................................................................................................................... vii

LIST OF FIGURES ....................................................................................................................... viii

1.0 Introduction ............................................................................................................................. 1

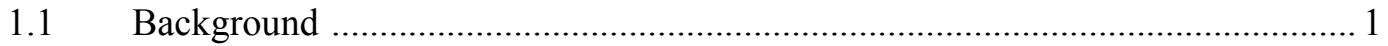

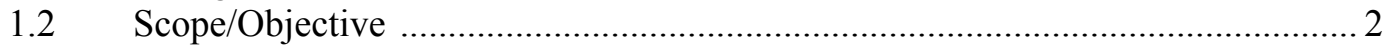

1.3 Thesis Organization .............................................................................

2.0 Literature Review ....................................................................................................... 4

$2.1 \quad$ The Adequacy of Code Eccentricity ................................................................. 4

2.2 Need for Realistic Modeling .................................................................... 9

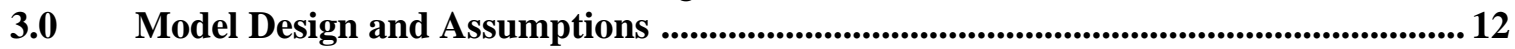

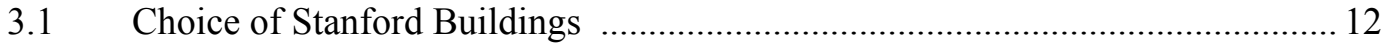

$3.2 \quad$ Modeling in OpenSees ............................................................................ 13

3.2.1 Simplification of Numerical Models ……………………………….... 13

3.2.2 Determination of Torsional Stiffness Coefficient for Columns ………... 14

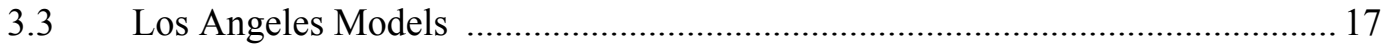

3.3.1 Three-Story Los Angeles, Post Northridge Model …………………..... 17

3.3.2 Nine-Story Los Angeles, Post Northridge Model ……………………..... 18

3.3.3 Twenty-Story Los Angeles, Post Northridge Model ………………….... 19

3.3.4 Seismic Model Assumptions ………………………………………......2.

$3.4 \quad$ Model Convergence …………………………………………………..... 21

3.4.1 Number of Integration Points vs. Number of Elements ………………...2 21

3.4.2 Force-Based Elements vs. Displacement-Based Elements ……………...2 23

3.5 Floor Load Parameters: 3-, 9-, and 20-Story Models ........................................ 29

3.5.1 Determination of Floor Loads ............................................................ 29

3.5.2 Monte Carlo Simulation: Effective Load Intensity and Location ............31

3.5.3 Seismic Mass Assignment ……………………………………………...... 34

4.0 Model Validation ...................................................................................................... 36

4.1 Translational Period Comparison ……………………………………………..... 36

4.1.1 Validation of Three-Story Model …………………………………..... 37

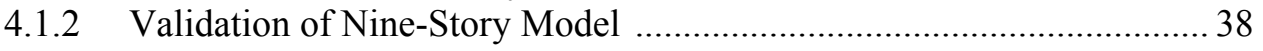

4.1.3 Validation of Twenty-Story Model ......................................................... 39

4.2 Additional Period Analysis .............................................................................. 39

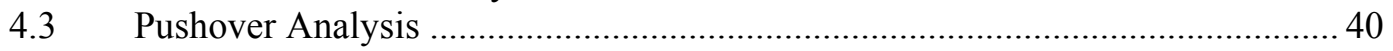

5.0 Validation of Probabilistic Sampling Method .................................................................... 43

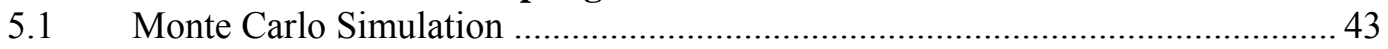

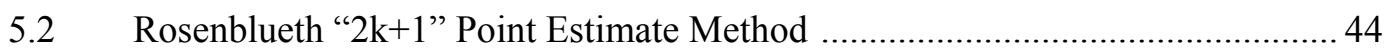

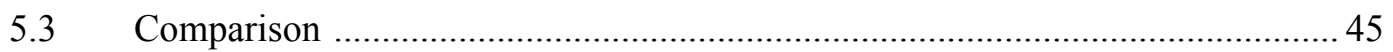

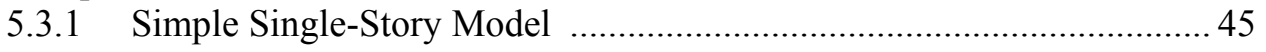

5.3.2 Simple Three-Story Model …………………………………………. 48

6.0 Parametric Study .......................................................................................................... 52

$6.1 \quad$ Ground Motions: Normalization ……………………................................... 52

6.2 Floor Load Intensities and Load Eccentricities ……………………………..... 55

6.3 Computer Specifications, Computational Time, and Residual Tolerance ........... 55

7.0 Results and Analysis ...................................................................................................59

7.1 Kolmogorov-Smirnov Test for Goodness-of-Fit: LogNormal vs. Normal ..........59

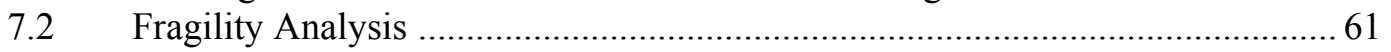

7.2.1 Fragility Analysis: Smooth/Regression Analysis .................................. 62

7.2.2 Fragility Analysis: Rough/Non-regression Analysis ............................... 63

7.2.3 Fragility Analysis: Comparison ……………………………………..... 64 


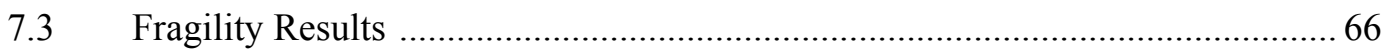

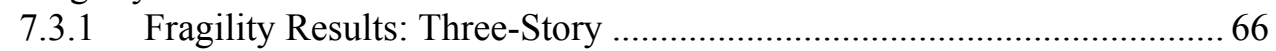

7.3.2 Fragility Results: Nine-Story ………………………………………........ 69

7.3.3 Fragility Results: Twenty-Story ……………………………………. 72

7.4 Collapse Margin Ratio ………………………………………………….... 74

7.5 Evaluation of Code Provisions for Consideration of Reactive Weight

Eccentricity ………………………………………………………….... 75

7.6 Building Performances Under DBE and MCE Events ....................................... 77

7.7 Performance Based Evaluation: FEMA 350 ………………………………..... 78

7.7.1 Determination of Confidence Levels .................................................... 80

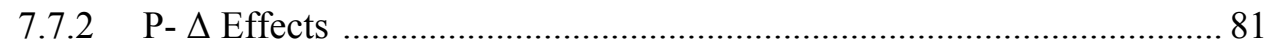

7.7.3 Comparison to Regression Fragility Curves ………………………….... 81

8.0 Conclusions and Recommendations for Future Research ............................................. 85

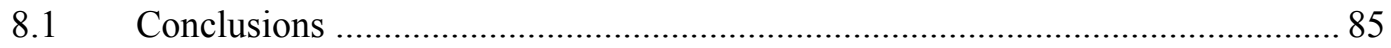

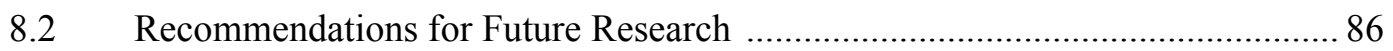

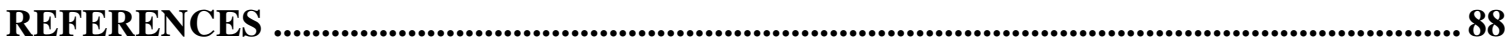

APPENDIX A: Model Layout, Floor Loads, and Mode Shapes ...................................................90

APPENDIX B: Disqualification of Particular Ground Motions ...................................................96

APPENDIX C: USGS Detailed Report ..............................................................................................98

APPENDIX D: Drift Results .......................................................................................................... 104

APPENDIX E: Fragility Curves .................................................................................................... 122

\section{LIST OF TABLES}

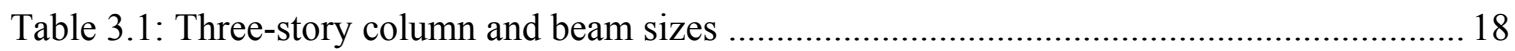

Table 3.2: Nine-story column and beam sizes ................................................................... 19

Table 3.3: Twenty-story column and beam sizes ............................................................... 20

Table 3.4: Fundamental period of simple single-story model, seconds ....................................... 22

Table 3.5: Maximum story drift of simple single-story model ……………………………….....2 23

Table 3.6: DBE vs FBE comparison along primary direction ................................................. 26

Table 3.7: DBE vs FBE comparison along minor direction ....................................................... 28

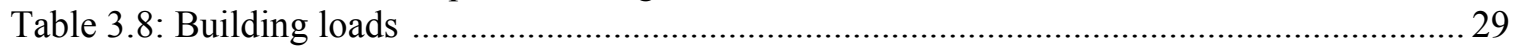

Table 3.9: Mass comparison: Stanford vs. hand calculated .......................................................... 30

Table 3.10: Intermediate floors for 3-, 9-, and 20-story parameters ........................................... 34

Table 4.1: Three-Story periods, seconds …………………………………………………........ 37

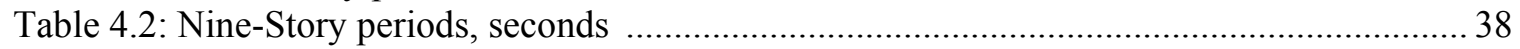

Table 4.3: Twenty-Story periods, seconds ………………………………………………........ 39

Table 5.1: Simple single-story model sampling method comparison .......................................... 48

Table 5.2: Simple three-story model sampling method comparison ………………………….... 50

Table 5.3: Required Rosenblueth " $2 \mathrm{k}+1$ " simulations for each ground motion .............................. 51

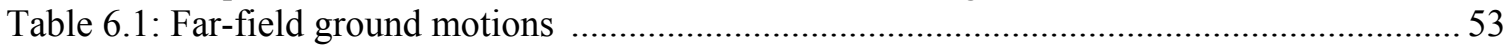

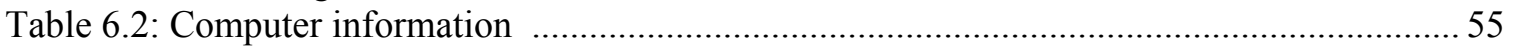

Table 6.3: Required simulation computational time ………………………………………....55

Table 6.4: Model complexity information …………………………………………...... 56

Table 7.1: Collapse margin ratios, CMR ……………………………………………….... 74

Table 7.2: Code drift normalized by Rosenblueth " $2 \mathrm{k}+1$ " drift ................................................. 76

Table 7.3: Probability of exceeding immediate occupancy drift limit .......................................... 77 


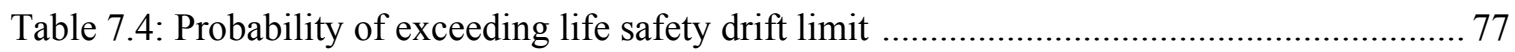

Table 7.5: Probability of exceeding collapse prevention drift limit .............................................. 78

Table A.1: Intermediate floors for 3-,9-, and 20-story load parameters ................................. 91

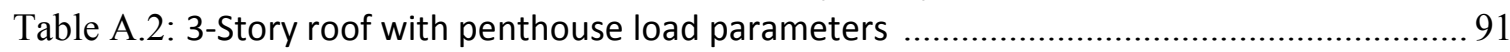

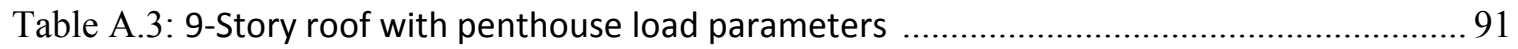

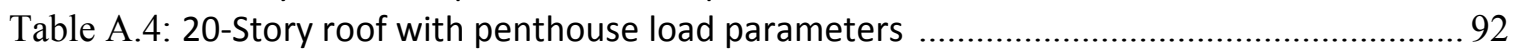

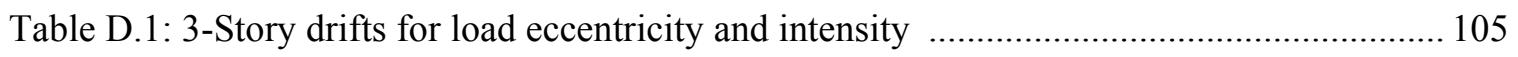

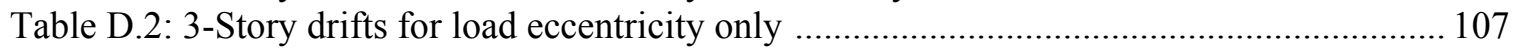

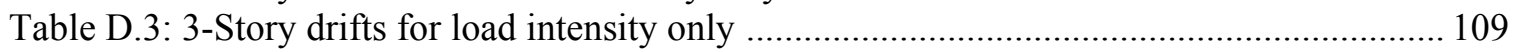

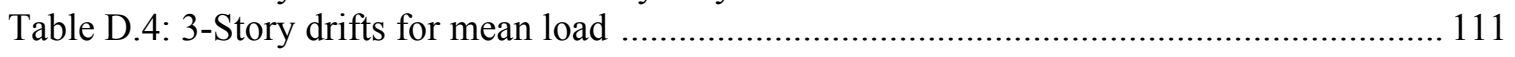

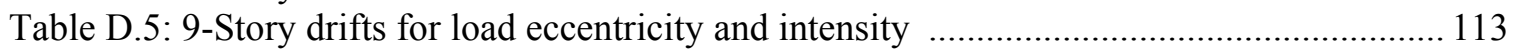

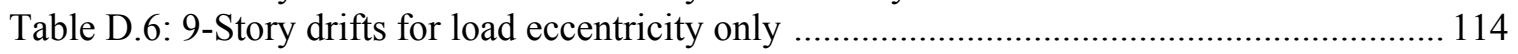

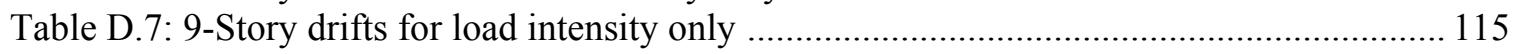

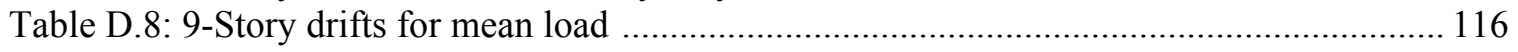

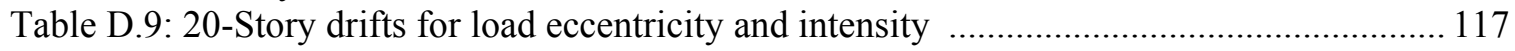

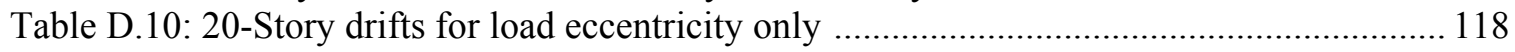

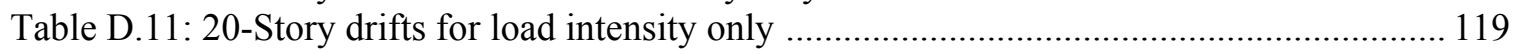

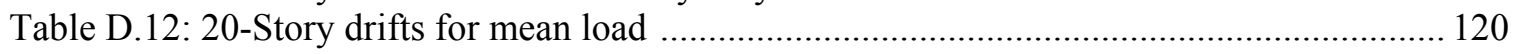

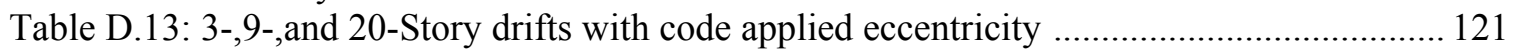

\section{LIST OF FIGURES}

Figure 2.1: Torsional amplification vs. coupling ratio, from Tso et al. (1980) ............................. 4

Figure 2.2: Design eccentricity code comparison, from Tso et al. (1980) ..................................... 5

Figure 2.3: Frequency distribution of normalized eccentricity and the assumed normal

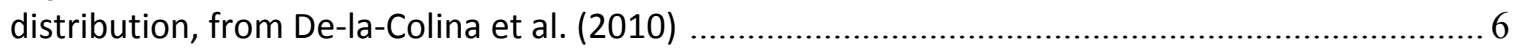

Figure 2.4: Fragility curves for 6 -story building at $2.5 \%$ drift limit, from Chang et al. (2009) ....... 7

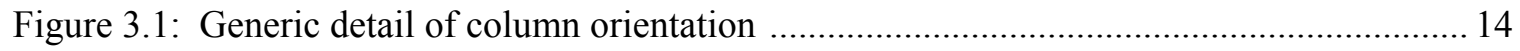

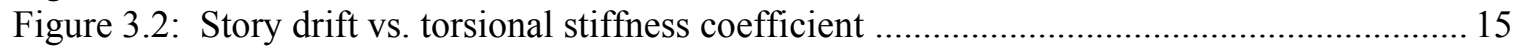

Figure 3.3: Fundamental period vs. torsional stiffness coefficient .......................................... 16

Figure 3.4: Kocæli Turkey primary component acceleration time history .................................. 24

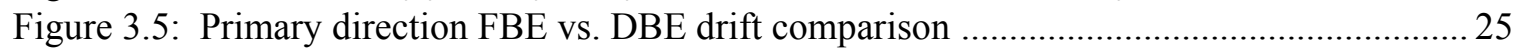

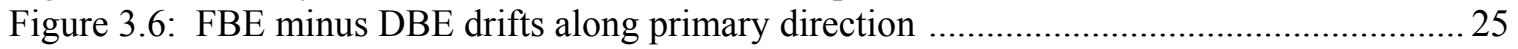

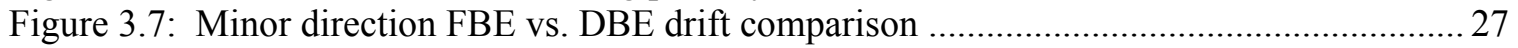

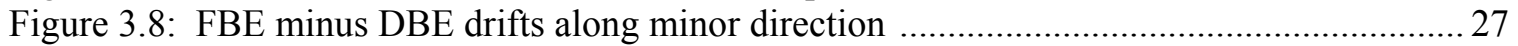

Figure 3.9: Convergence of effective mean load intensity, lower floors .................................... 32

Figure 3.10: Convergence of effective load intensity standard deviation, lower floors ............... 33

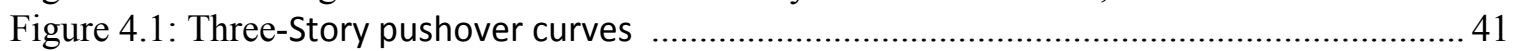

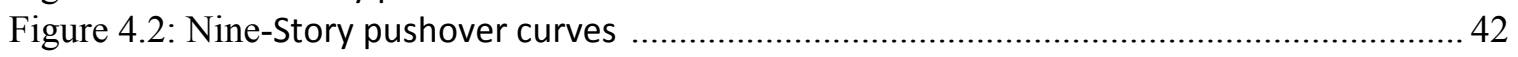

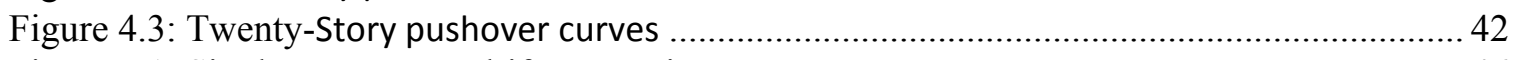

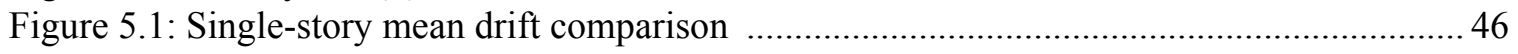

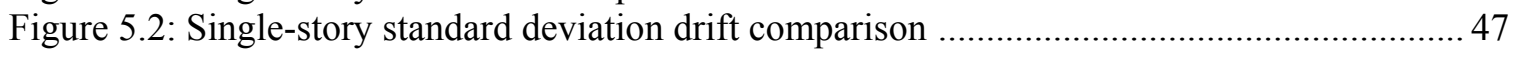

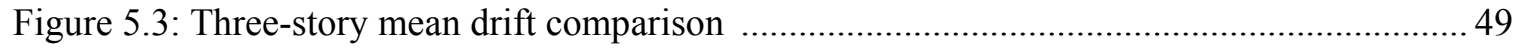

Figure 5.4: Three-story standard deviation drift comparison .................................................. 50 
Figure 6.1: Northridge (Station: Mulhol) spectral acceleration components ............................... 54

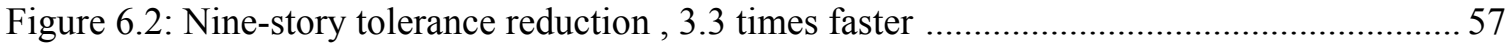

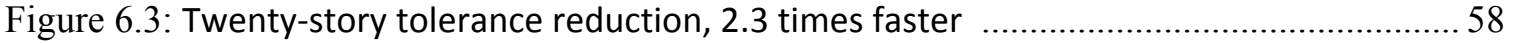

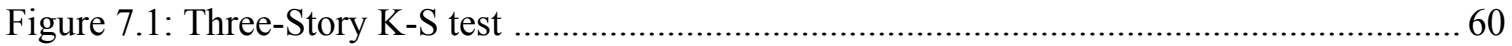

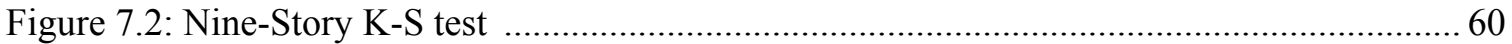

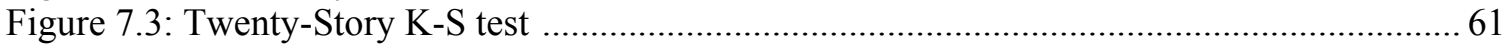

Figure 7.4: Regression fragility curves for load eccentricity and intensity, 3-story .................... 63

Figure 7.5: Non-regression fragility curves for load eccentricity and intensity, 3-story .............. 64

Figure 7.6: Regression and non-regression fragility curve comparison, 3 -story .........................6 65

Figure 7.7: Regression fragility curves for immediate occupancy comparison, 3-story ................67 67

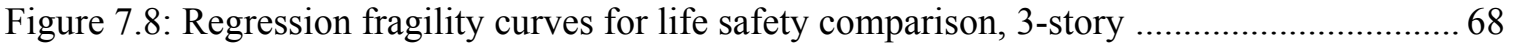

Figure 7.9: Regression fragility curves for collapse prevention comparison, 3 -story …................ 68

Figure 7.10: Regression fragility curves for immediate occupancy comparison, 9-story ….......... 70

Figure 7.11: Regression fragility curves for life safety comparison, 9-story .............................. 71

Figure 7.12: Regression fragility curves for collapse prevention comparison, 9-story ............... 71

Figure 7.13: Regression fragility curves for immediate occupancy comparison, 20-story .......... 72

Figure 7.14: Regression fragility curves for life safety comparison, 20-story ............................ 73

Figure 7.15: Regression fragility curves for collapse prevention comparison, 20-story .............. 73

Figure 7.16: FEMA fragility curves, with code capacity and reduced capacity, compared to

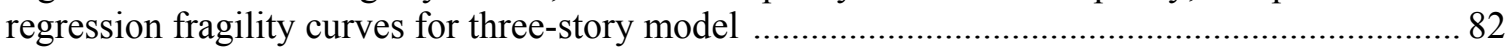

Figure 7.17: FEMA fragility curves, with code capacity and reduced capacity, compared to

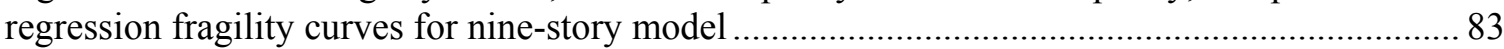

Figure 7.18: FEMA fragility curves, with code capacity and reduced capacity, compared to

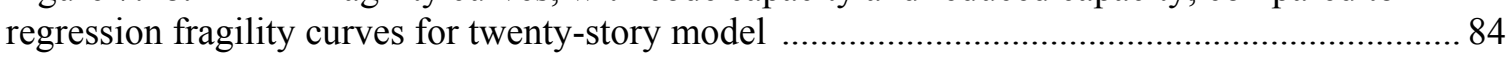

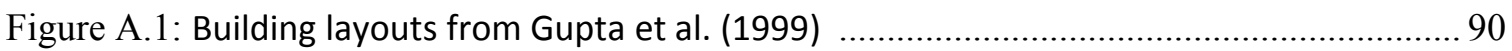

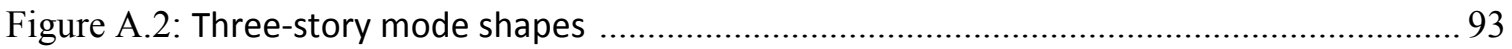

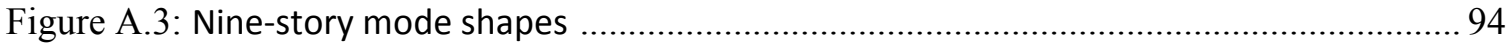

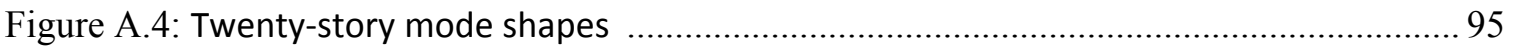

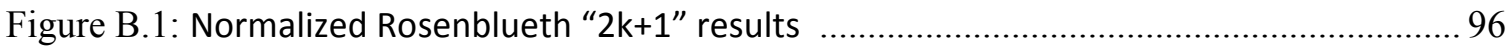

Figure E.1: Non-regression fragility curves for load eccentricity and intensity, 3-story ............ 123

Figure E.2: Non-regression fragility curves for load eccentricity, 3-story ................................. 123

Figure E.3: Non-regression fragility curves for load intensity, 3-story .................................... 124

Figure E.4: Non-regression fragility curves immediate occupancy comparison, 3-story .......... 124

Figure E.5: Non-regression fragility curves life safety comparison, 3-story ............................ 125

Figure E.6: Non-regression fragility curves collapse prevention comparison, 3-story .............. 125

Figure E.7: Regression fragility curves for load eccentricity and intensity, 3-story .................. 126

Figure E.8: Regression fragility curves for load eccentricity, 3-story ...................................... 126

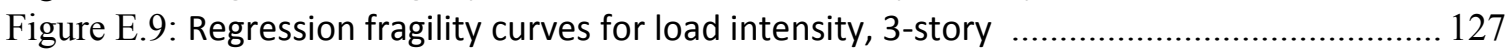

Figure E.10: Regression fragility curves for mean values of eccentricity and intensity, 3-story 127

Figure E.11: Regression fragility curves for immediate occupancy comparison, 3-story .......... 128

Figure E.12: Regression fragility curves for life safety comparison, 3-story ............................ 128

Figure E.13: Regression fragility curves for collapse prevention comparison, 3-story .............. 129

Figure E.14: Regression and non-regression fragility curve comparison for load eccentricity

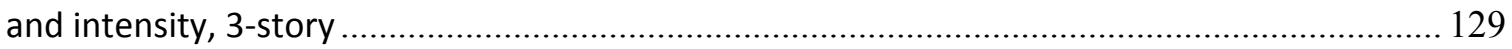

Figure E.15: Regression and non-regression fragility curve comparison for load eccentricity, 3-story 
Figure E.16: Regression and non-regression fragility curve comparison for load intensity,

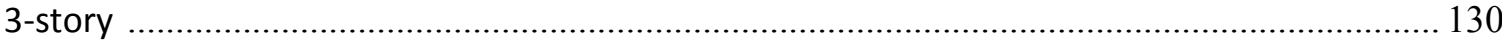

Figure E.17: Regression and FEMA comparison for load eccentricity and intensity, 3-story .... 131

Figure E.18: Regression and FEMA comparison for load eccentricity, 3-story .......................... 131

Figure E.19: Regression and FEMA comparison for load intensity, 3-story .............................. 132

Figure E.20: FEMA 350 fragility curves for immediate occupancy comparison, 3-story ........... 133

Figure E.21: FEMA 350 fragility curves for collapse prevention comparison, 3-story ............... 133

Figure E.22: Non-regression fragility curves for load eccentricity and intensity, 9-story .......... 134

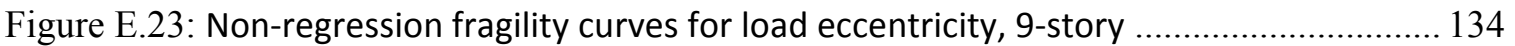

Figure E.24: Non-regression fragility curves for load intensity, 9-story ................................... 135

Figure E.25: Non-regression fragility curves immediate occupancy comparison, 9-story ......... 135

Figure E.26: Non-regression fragility curves life safety comparison, 9-story ........................... 136

Figure E.27: Non-regression fragility curves collapse prevention comparison, 9-story ............ 136

Figure E.28: Regression fragility curves for load eccentricity and intensity, 9-story ................. 137

Figure E.29: Regression fragility curves for load eccentricity, 9-story ..................................... 137

Figure E.30: Regression fragility curves for load intensity, 9-story .......................................... 138

Figure E.31: Regression fragility curves for mean values of eccentricity and intensity, 9-story 138

Figure E.32: Regression fragility curves for immediate occupancy comparison, 9-story .......... 139

Figure E.33: Regression fragility curves for life safety comparison, 9-story .............................. 139

Figure E.34: Regression fragility curves for collapse prevention comparison, 9-story .............. 140

Figure E.35: Regression and non-regression fragility curve comparison for load eccentricity

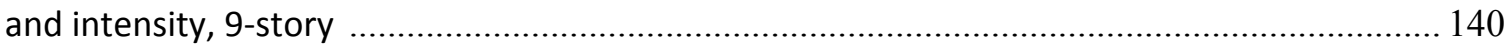

Figure E.36: Regression and non-regression fragility curve comparison for load eccentricity,

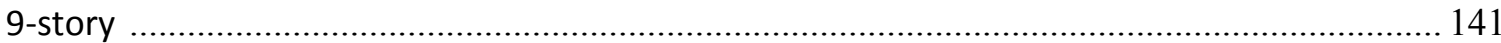

Figure E.37: Regression and non-regression fragility curve comparison for load intensity,

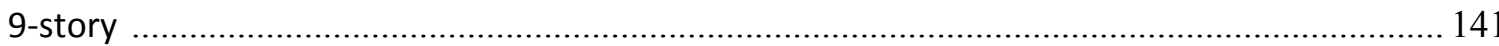

Figure E.38: Regression and FEMA comparison for load eccentricity and intensity, 9-story .... 142

Figure E.39: Regression and FEMA comparison for load eccentricity, 9-story .......................... 142

Figure E.40: Regression and FEMA comparison for load intensity, 9-story .............................. 143

Figure E.41: FEMA 350 fragility curves for immediate occupancy comparison, 9-story ........... 144

Figure E.42: FEMA 350 fragility curves for collapse prevention comparison, 9-story ............... 144

Figure E.43: Non-regression fragility curves for load eccentricity and intensity, 20-story ........ 145

Figure E.44: Non-regression fragility curves for load eccentricity, 20-story .............................. 145

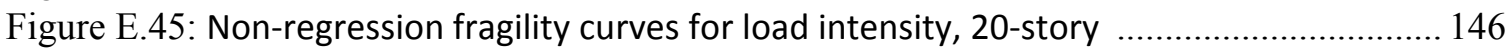

Figure E.46: Non-regression fragility curves immediate occupancy comparison, 20-story ...... 146

Figure E.47: Non-regression fragility curves life safety comparison, 20-story ......................... 147

Figure E.48: Non-regression fragility curves collapse prevention comparison, 20-story .......... 147

Figure E.49: Regression fragility curves for load eccentricity and intensity, 20-story ............... 148

Figure E.50: Regression fragility curves for load eccentricity, 20-story .................................. 148

Figure E.51: Regression fragility curves for load intensity, 20-story ....................................... 149

Figure E.52: Regression fragility curves for mean values of eccentricity and intensity,

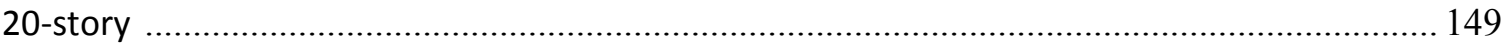

Figure E.53: Regression fragility curves for immediate occupancy comparison, 20-story ........ 150

Figure E.54: Regression fragility curves for life safety comparison, 20-story .............................. 150

Figure E.55: Regression fragility curves for collapse prevention comparison, 20-story ............ 151

Figure E.56: Regression and non-regression fragility curve comparison for load eccentricity

and intensity, 20-story 
Figure E.57: Regression and non-regression fragility curve comparison for load eccentricity, 20-story .....

Figure E.58: Regression and non-regression fragility curve comparison for load intensity,

20-story

Figure E.59: Regression and FEMA comparison for load eccentricity and intensity, 20-story .. 153

Figure E.60: Regression and FEMA comparison for load eccentricity, 20-story ........................ 153

Figure E.61: Regression and FEMA comparison for load intensity, 20-story ........................... 154

Figure E.62: FEMA 350 fragility curves for immediate occupancy comparison, 20-story ......... 155

Figure E.63: FEMA 350 fragility curves for collapse prevention comparison, 20-story 155 


\section{Chapter 1: Introduction}

\subsection{Background}

When a building is subjected to seismic excitation it exhibits both a translational response and a torsional response. There are many factors that account for and produce the torsional response. They fall into two categories: natural and accidental eccentricities. A natural eccentricity is due to the effective center of mass not coinciding with the center of rigidity at the floor level of interest. Natural eccentricities are often a result of irregular weight and/or stiffness distributions in the structures. An accidental eccentricity attempts to account for the uncertainty of the actual mass centers, the centers of rigidity, the member strengths, the rotational ground motions, and the premature yielding of members in the lateral force resisting system (FEMA 350, 2000). The natural eccentricities are evident in the design and are generally attempted to be minimized to reduce the torsion. The accidental eccentricity is the unknown variable and is prescribed as $5.0 \%$ of the plan dimension of the building floor perpendicular to the direction of excitation in the IBC, CBC, and NEHRP. For the Mexico City Building Code and the National Building Code of Canada it is prescribed as $10.0 \%$ of the plan dimension (Fahjan et al., 2006).

The code defined accidental eccentricity values of $5.0 \%$ or $10.0 \%$ are arbitrary values and represent the minimum amount allowed by their respective codes. Some studies have been done to better quantify the accidental eccentricity and evaluate the code provisions. De-la-Colina et al. (2011) surveyed actual office buildings in Mexico City and suggested through a Monte Carlo Simulation that the probability of exceeding $5.0 \%$ or $10.0 \%$ is minimal and only likely at the top floors and that the probability of exceedance decreases significantly for lower stories.

Though the prior research that has been done by randomly applying the code prescribed $5.0 \%$ eccentricity along the plan dimensions, to the best of this author's knowledge, there has not been 
much research done on evaluation of seismic performance of steel moment frame buildings (particularly seismic fragilities) with the use of realistic reactive weights with random acting locations and amplitudes.

\subsection{Scope/Objective}

The objective of this research was first to quantify the torsional effects in the selected steel building frames caused by the accidental eccentricity due to the dead and live load variability of intensity and location, and second to compare the results to those produced from a code applied eccentricity analysis and comment on the adequacy of the code. Three steel moment resisting frames structures were selected from existing literature and modeled in this thesis. These structures are representative of a low-rise, a mid-rise, and a high-rise office building. To ensure credible assumptions and accurately capture the effects of eccentric loading, all of the buildings were modeled in $3 \mathrm{D}$ and excited with bi-directional ground motions. Using actual surveyed dead and live reactive weights, an extensive parametric study was performed using nonlinear response history analyses to determine the effect of uncertain acting location and amplitude of the floor loads on seismic performance of the buildings.

\subsection{Thesis Organization}

This thesis includes a total of eight chapters to address the random load eccentricity and amplitude issues presented above. It is organized to provide the reader with a sequential process of how the research was conducted. 
Chapter 2 provides an overview of past research related to building torsional response induced from eccentric loading. The review also includes types of modeling as well as the types of variables considered for analysis.

Chapter 3 describes basic information of the selected building and detailed modeling process of these buildings in Open System for Earthquake Engineering Simulation (OpenSees).

Chapter 4 presents validation of the OpenSees models based on the published results of the selected buildings.

Chapter 5 provides the details and validation of the considered probabilistic sampling method which was used to generate the random reactive weights at floor levels.

Chapter 6 describes the particular variables and their ranges that were considered in the parametric analysis as well as the specifications for the computers used to perform the simulations.

Chapter 7 interprets the results from the parametric analysis.

Chapter 8 summarizes the results from Chapter 7 and provides recommendations for future research. 


\subsection{The Adequacy of Code Eccentricity}

Tso et al. (1980)

Tso et al. (1980) used a single-story single mass system to evaluate the adequacy of several code provisions in considering the torsional effects in buildings caused by seismic loading. The torsional results of the single-story coupled system are combinable with those of a multistory uncoupled system to develop the torsional results for certain multistory buildings. Regardless of the mass eccentricity applied to the single-story system, the torsional amplification was maximized at a torsional to lateral frequency equal to unity, seen in Fig. 2.1.

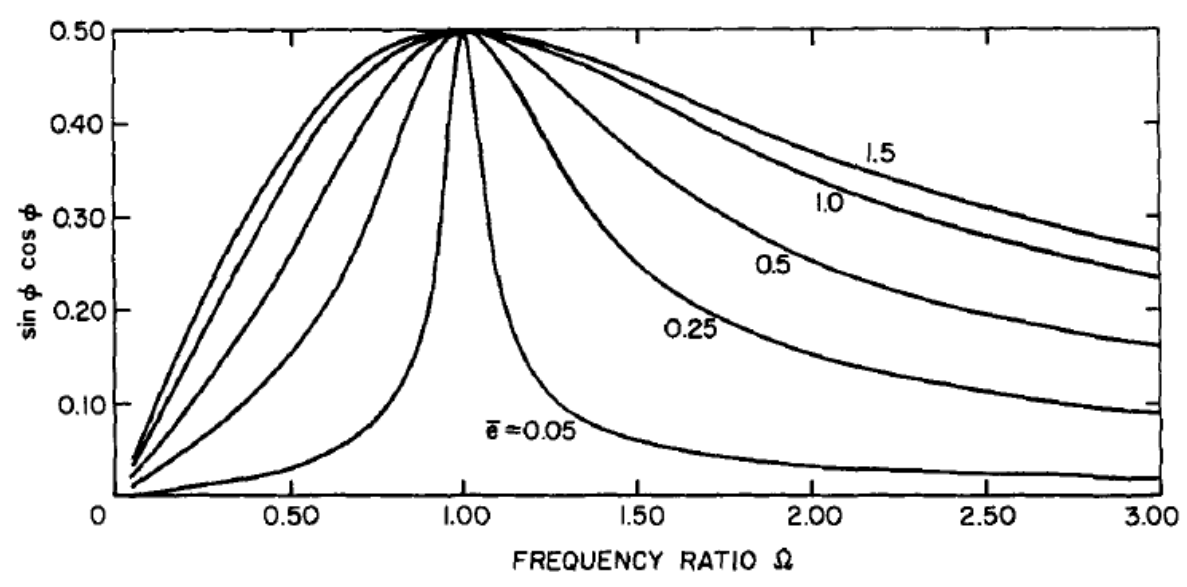

Figure 6. Effect of $\Omega$ on $\sin \phi \cos \phi$

Figure 2.1: Torsional amplification vs. coupling ratio, from Tso et al. (1980)

The system was tested over the full range of eccentricities and compared to the provisions of several different seismic codes. It was determined that at smaller eccentricities, less than $12.5 \%$, all of the code provisions under-estimated the values. The comparison to the code results is seen in Fig. 2.2 below. 


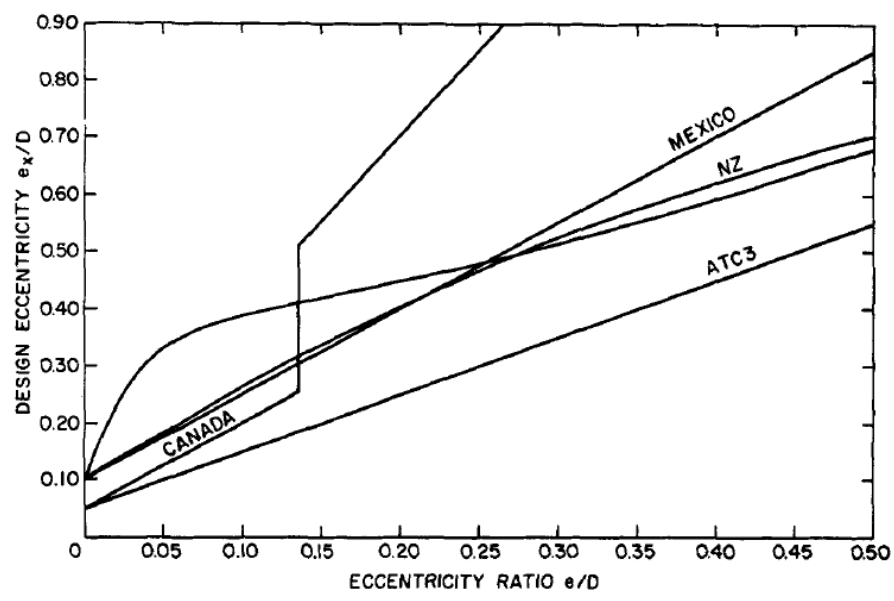

Figure 12. Comparison of design eccentricities with building code provisions

Figure 2.2: Design eccentricity code comparison, from Tso et al. (1980)

Though it appears that the torsion is being grossly underestimated by the codes, these values only correspond to the torsional response which is added to the translational response. When the translational response is much greater, the additive effects of the torsion are minimized.

\section{De-la-Colina et al. (2011)}

De-la-Colina et al. (2011) investigated how the variability of different random variables affects the probability distribution of accidental eccentricity. Previous studies performed by De la Llera and Chopra, Escobar, and De-la-Colina and Almeida investigated the effects of member stiffness and eccentricities, but none of the studies were performed with actual surveyed loads. Therefore, a survey was conducted in Mexico City to obtain accurate dead and live load intensities and locations for more realistic analysis. The survey recorded results from office buildings and concluded that the instantaneous live load was best fit with a gamma probability distribution function, and its position was best fit with a Gaussian probability density function. The dead load intensity and position were determined to be best represented with a Gaussian probability distribution. 
The surveyed data was applied in a Monte Carlo Simulation to both a five-story and a ten-story model. The accidental eccentricities at each floor were recorded for each simulation and used to produce the histograms in Fig. 2.3 below.
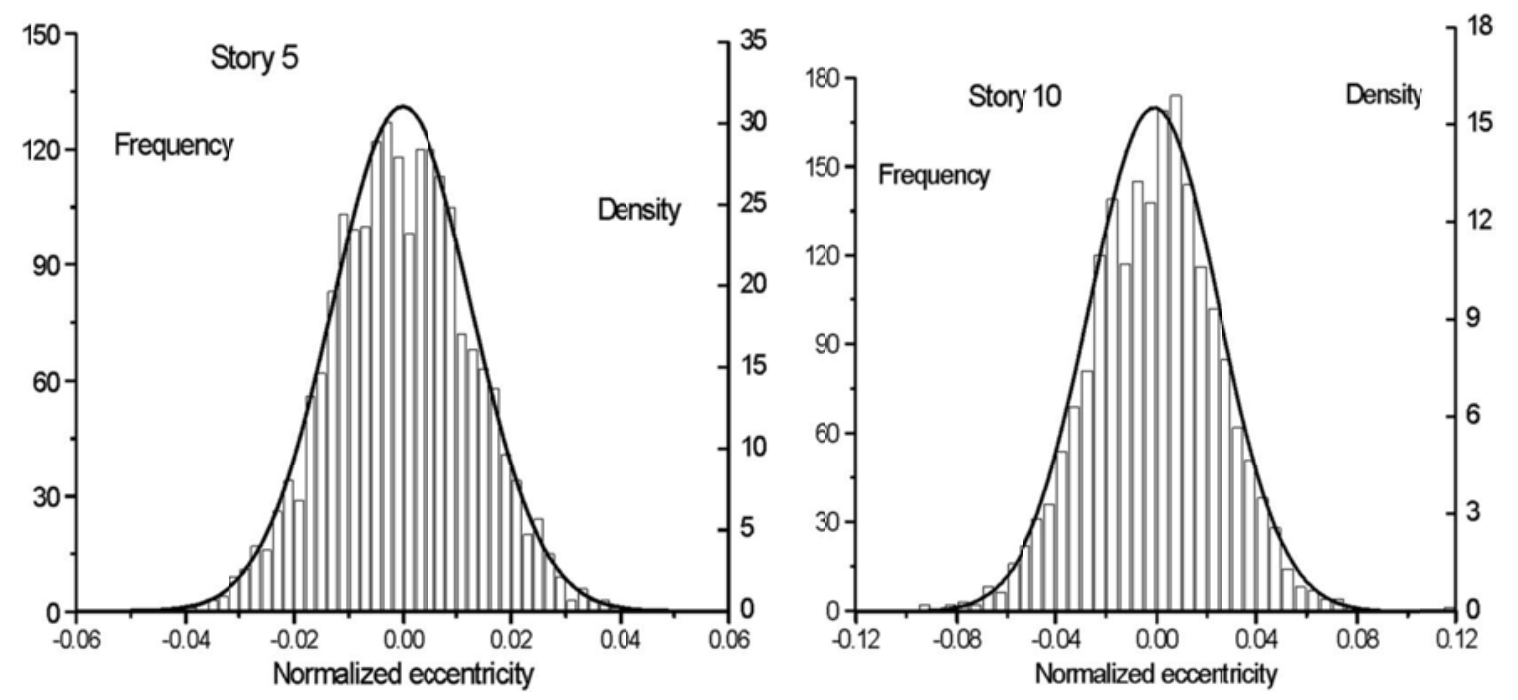

Fig. 2.3: Frequency distribution of normalized eccentricity and the assumed normal distribution, from De-la-Colina et al. (2011)

The above histogram shows the effective accidental eccentricities at floors five and ten, respectively, within the ten-story model. As expected the average accidental eccentricity is essentially zero at each floor. The distribution, however, is smaller for the fifth floor because the effective eccentricity is a product of all the random eccentricities applied to the floors above. It was also determined that as the number of lateral force resisting elements per floor increased the accidental eccentricity decreased for the same reason.

De-la-Colina et al. concluded that the probability of exceeding a $5.0 \%$ accidental eccentricity is only $1.0 \%$ at the upper levels and that probability decreases considerably as the number of floors above increases. 


\section{Chang et al. (2009)}

Chang et al. (2009) performed fragility analyses on six-story and twenty-story steel moment resisting frame office buildings. The use of realistic models minimized the uncertainties and variability associated with an oversimplified single-story model. The masses at each floor were applied at $5.0 \%$ of the plan dimension according to the Taiwan building code. The eccentricities were applied along the longer plan dimension, which is perpendicular to the uni-directional ground motion. Three different loading scenarios were considered: no eccentricities (MRF), code eccentricities at each floor (MRF_EC), and Latin Hypercube Sampling of code eccentricities (MRF_REC). The code applied eccentricities were applied in the same direction for each floor, while the Latin Hypercube Sampling randomly applied the eccentricities 5.0\% to either side or not at all in order to mimic the random nature of loading.

Fragility curves were generated for two limit states: immediate occupancy and life safety described in FEMA 356 as $0.7 \%$ and $2.5 \%$ story drifts, respectively, for steel moment resisting frames.

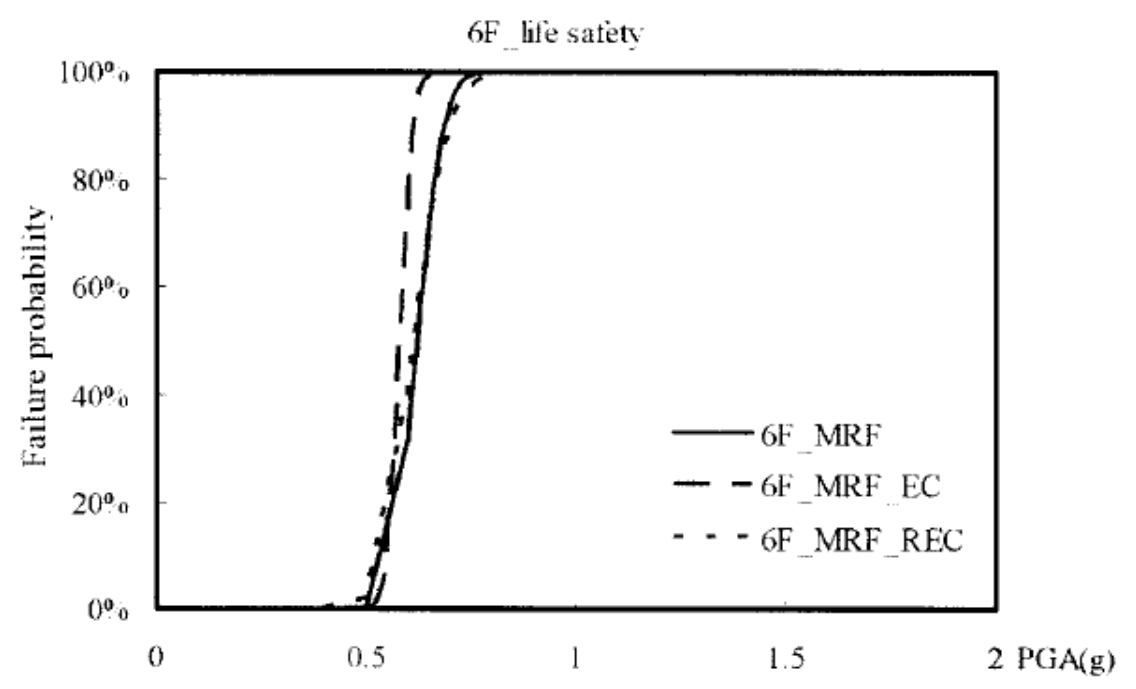

Figure 2.4: Fragility curves for 6-story building at 2.5\% drift limit, from Chang et al. (2009) 
The main conclusion, which can be seen from the fragility curves in Fig 2.4 above, is that the code applied eccentricities overestimate the demands. The zero eccentricity case, labeled as "MRF" in the figure above, and the randomly applied eccentricity case, labeled as "MRF_REC," are very similar, whereas the code applied case is significantly more conservative.

\section{Stathopoulos et al. (2000)}

Stathopoulos et al. (2000) evaluated the effects of eccentricity on a single-story reinforced concrete shear beam model and a plastic hinge model. The effects of mass eccentricity and stiffness eccentricity, as well as single versus double eccentricity were evaluated. The peak ductility factors from a set of ground motions were averaged and used as the comparison index. For each type of model three subsequent models were also designed: mass and stiffness aligned with the geometric center, only mass aligned with the geometric center, and, finally, only stiffness aligned with the geometric center.

Results revealed that the differences caused by either mass eccentric or stiffness eccentric were quantitative. Qualitatively the results were essentially the same for these two different models. Again the results for the single and double applied eccentricity revealed minimal difference at lower reasonable levels of eccentricity. The ductility demands from the plastic hinge model suggest that the code provisions are adequate for a single-story reinforced concrete building, and that the design eccentricities had very little effect on the responses. 


\subsection{Need for Realistic Modeling}

\section{Stathopoulos et al. (2005)}

Stathopoulos et al. (2005) evaluated the accuracy of the oversimplified single-story shear beam model in torsional response. The simplified shear beam model does not account for the relationship between structure strength, stiffness, and yield deformation. It also neglects all post yield strength of yielded members, and the single-story system is unable to capture the higher mode effects of multistory buildings. Two reinforced-concrete spaced frame models (three-story and five-story) were excited with several different bi-directional semi-artificial earthquakes. The responses of these tests were averaged together to ensure that the results were not unique to a particular ground motion. Examining the ductility demands it was determined that for the most part the columns remained elastic while the beams experienced plastic behavior, as expected. Moreover, as the natural eccentricity increased, the demands on the flexible edge also increased while the ductility demands on the stiff edge decreased. In this case, the flexible edge refers to lateral force resisting element on the opposite side of the center of rigidity as the center of mass, and the stiff edge corresponds to those on the same side. The decrease in ductility demand along the stiff edge was not as significant as the increase along the flexible edge. This uneven distribution of ductility demands can cause local failures to occur and the seismic forces to become concentrated. These findings are in direct contrast to the simplified shear beam model and demand the use of more realistic models in analysis.

\section{Gupta et al. (1999)}

In the wake of the 1994 Northridge and 1995 Kobe earthquakes there was great concern in the behavior of steel moment resisting frames. The John A. Blume Earthquake Engineering Center, part of the Department of Civil and Environmental Engineering at Stanford University, conducted 
extensive research into steel moment resisting frames. Three buildings, representative of a lowrise, mid-rise, and high-rise structure (three-story, nine-story, and twenty-story, respectively), where designed by prominent consulting engineering companies in three geographically diverse locations in the United States (Los Angeles, Seattle, and Boston). 2D models were used to analyze local and global behavior, demand predictions, the effects of different material properties, and the effects of different types of connections. These three models are representative office buildings in their respective geographical regions and the Los Angeles designs will serve as the basis for the research conducted in this thesis.

\section{Fahjan et al. (2006)}

Fahjan et al. (2006) proposed the Multi-Modal Eccentricity dynamic analysis procedure.

Traditionally a fully dynamic analysis would have to be performed for each eccentric loading scenario. This procedure is computationally intensive and requires a lot of time. The proposed method combines the global force vectors for various scenarios to develop an effective global force vector. The nodal displacements and member forces can be easily determined from the force vectors. For the proposed method the traditional procedures are employed to determine the global force vectors of the zero eccentricity case. These vectors are then modified to represent the particular code determined eccentricity force vector. These vectors can then be combined by any desired modal combination technique: complete quadratic combination, square root of sum of squares, etc. Enough mode shapes are considered until $90.0 \%$ of the total mass is participating. This procedure only requires one dynamic analysis to be performed and the modified global force vectors are simply substituted back into the equation for each eccentricity case. 
This method produced slightly more conservative, but comparable, values over the previous methods. This will allow for more realistic models to be created and used without the need to run such intensive analysis for each loading case. 


\section{Chapter 3: Model Design and Assumptions}

\subsection{Selection of Steel Moment Frame Buildings}

This research focuses on investigation of response of steel moment frame buildings subjected to uncertain distribution of floor masses and earthquake excitations. As such, the selected steel moment frames should represent the current design and construction practice in regions with moderate and high seismicity. Since the last century, pioneering researchers from the Department of Civil and Environmental Engineering at Stanford University (Gupta et al. 1999) have been working on seismic performance evaluation of a series of steel moment frames designed based on the performance design framework recently developed through the SAC project. Specifically, their models include nine steel moment frames, which include three different steel moment resisting frame structures (a three-story low-rise, a nine-story mid-rise, and a twenty-story highrise) respectively designed for three areas with different levels of seismicity (i.e., Los Angeles, Seattle, and Boston). As described in Gupta et al. (1999), "the structural systems for these buildings have been designed by leading consulting engineering companies and are deemed to be representative of typical design practices followed currently in different parts of the US [Los Angeles, Seattle, and Boston]." The basic guidelines for the design of these structures are considered to be indicative of many steel moment resisting frame structures and, consequently, the buildings have been widely used as benchmark buildings in the earthquake engineering community for different research purposes. In this research, the three buildings designed for the Los Angeles area, which are believed to be a proper representation of the post-Northridge steel moment resisting frame office buildings in California, were selected for numerical modeling, parametric analysis, and probabilistic evaluation. It is noted that each of the three structures was designed as a typical office building for the Los Angeles area in accordance with UBC 1994 and 
FEMA 267 (1995), for post-Northridge seismic considerations. More detailed information about the selected building can be found in Gupta et al. (1999)

\subsection{Modeling in OpenSees}

For this thesis, numerical modeling of the three selected Los Angeles office buildings was completed in OpenSees, which is a script based computer program that allows for the simple implementation of a parametric analysis with software such as MATLAB.

\subsubsection{Simplification of Numerical Models}

All three of the Los Angeles models are based on the OpenSees' example file: Example7. 3D Moment-Resisting Frame (W-section) written by Mazzoni and McKenna in 2006. This simple three story, single bay by single bay model was the basis for each of the three Los Angeles models. The geometries of the three Los Angeles office building structures are provided in Appendix A. To simplify the complexity of the models, the gravity frame elements, both columns and beams, were not explicitly modeled. Although the gravity frames do contribute to the lateral stiffness, they are generally neglected for these types of analyses (Gupta et al., 1999). By neglecting the contribution of the gravity system the demands on the lateral-force-resistingsystem will be overestimated. This overestimation is accounted for in the FEMA 350 Performance Based Analysis with demand factors which will be described in greater detail in Section 7.7. Therefore, only the moment resisting frames (as shown in green in Fig. 3.1 for the three-story model) were explicitly modeled in the numerical models.

Without the members as part of the gravity frames linking the individual moment frames, a rigid diaphragm is required. All of the nodes at each floor level were assigned to their own rigid 
diaphragm for each respective floor. The rigid diaphragm allows the members at each floor level to move in unison, which preserves the torsional motion, and allows for the seismic mass to be lumped to the four exterior corners of each floor as described in Section 3.5.3

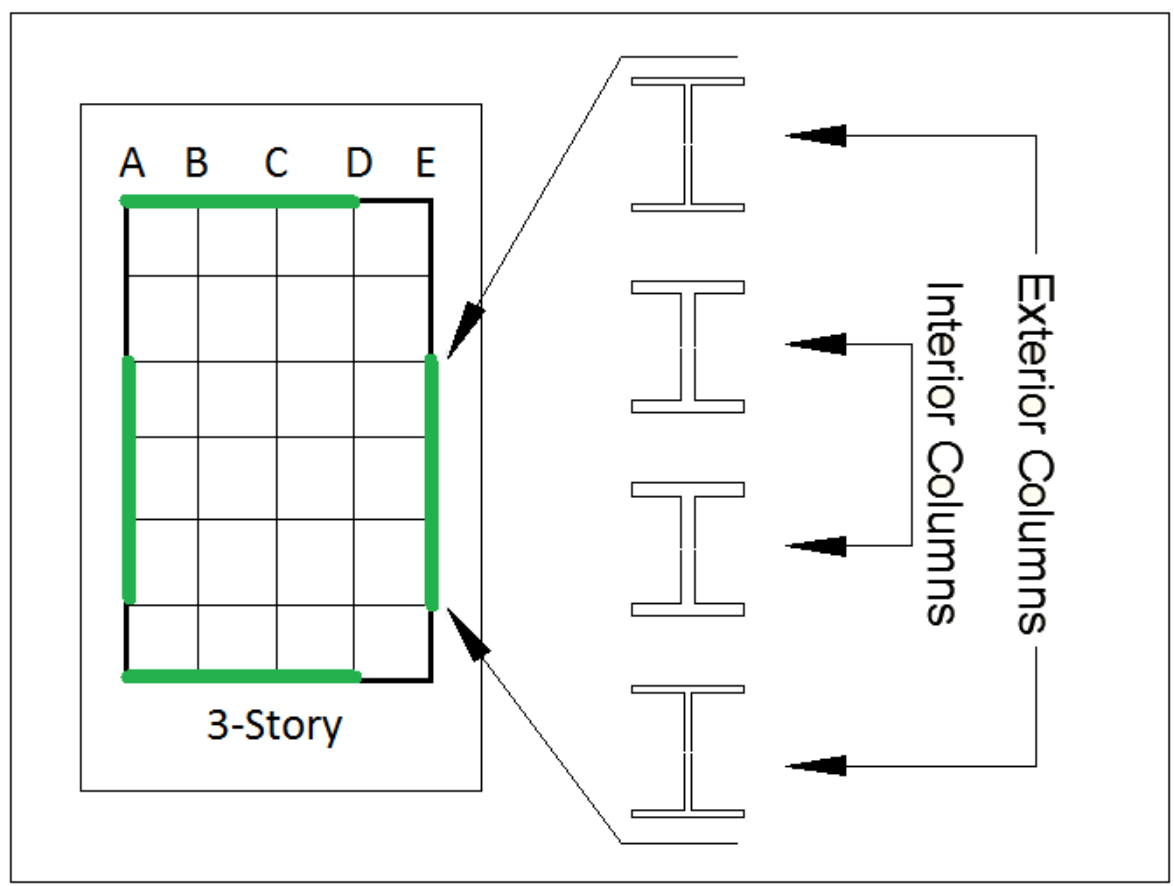

Figure 3.1: Generic detail of column orientation

\subsubsection{Determination of Torsional Stiffness Coefficient for Columns}

In addition to the three Los Angeles office building models, two more models were created in OpenSees and SAP2000 for testing and validation purposes: a single-story single bay by single bay model, and a three-story single bay by single bay model. Both of these simplified models were designed with column and beam sizes similar to the Los Angeles three story building. These simplified models facilitate parameter selection and reduce the computation time for the numerous simulations required to validate the sampling methods that are used on the full-scale computer models. 
The simplified models were analyzed in OpenSees with several uni-directional ground motions over the full range of mass eccentricities, $0-50 \%$ (where eccentricity is measured as a fraction of the plan dimension from the geometric center). It was revealed that the models were torsionally stiff and exhibited minimal rotational response, even at the maximum 50\% eccentricity scenario. In addition to the minimal rotation observed with the OpenSees model, the rotational period of the model was significantly less than the expected value generated from an identical SAP2000 model, again suggesting that the OpenSees model was torsionally stiff. The assigned torsional stiffness coefficient for the OpenSees 3D model was adjusted until the behavior of the model corresponded to the SAP2000 model. The orthogonal story drifts were evaluated over a range of stiffness coefficients. The orthogonal story drifts are a measure of the torsion being produced because the models are only being excited by a single uni-directional ground motion. Figs. 3.2 and 3.3 show the results of the parameter sweep for orthogonal drifts and rotational periods, respectively.

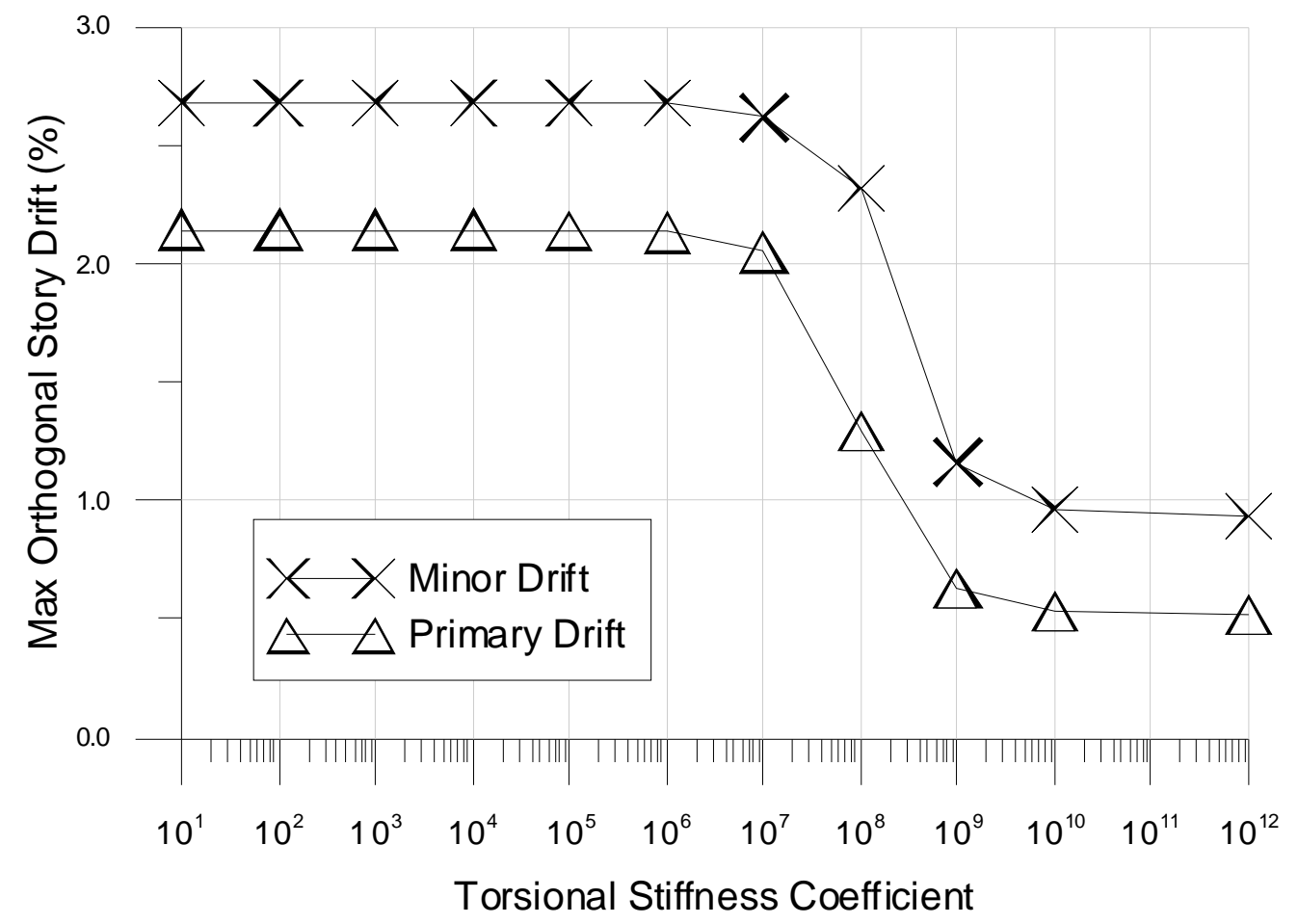

Figure 3.2: Story drift vs. torsional stiffness coefficient 
Fig. 3.2 illustrates how the model responds to the torsional stiffness coefficient. As the coefficient is decreased the model becomes free to develop rotation about its vertical axis. At a torsional stiffness coefficient of about $1.0 \mathrm{e} 06$ the orthogonal story drifts converge to respective maximum values.

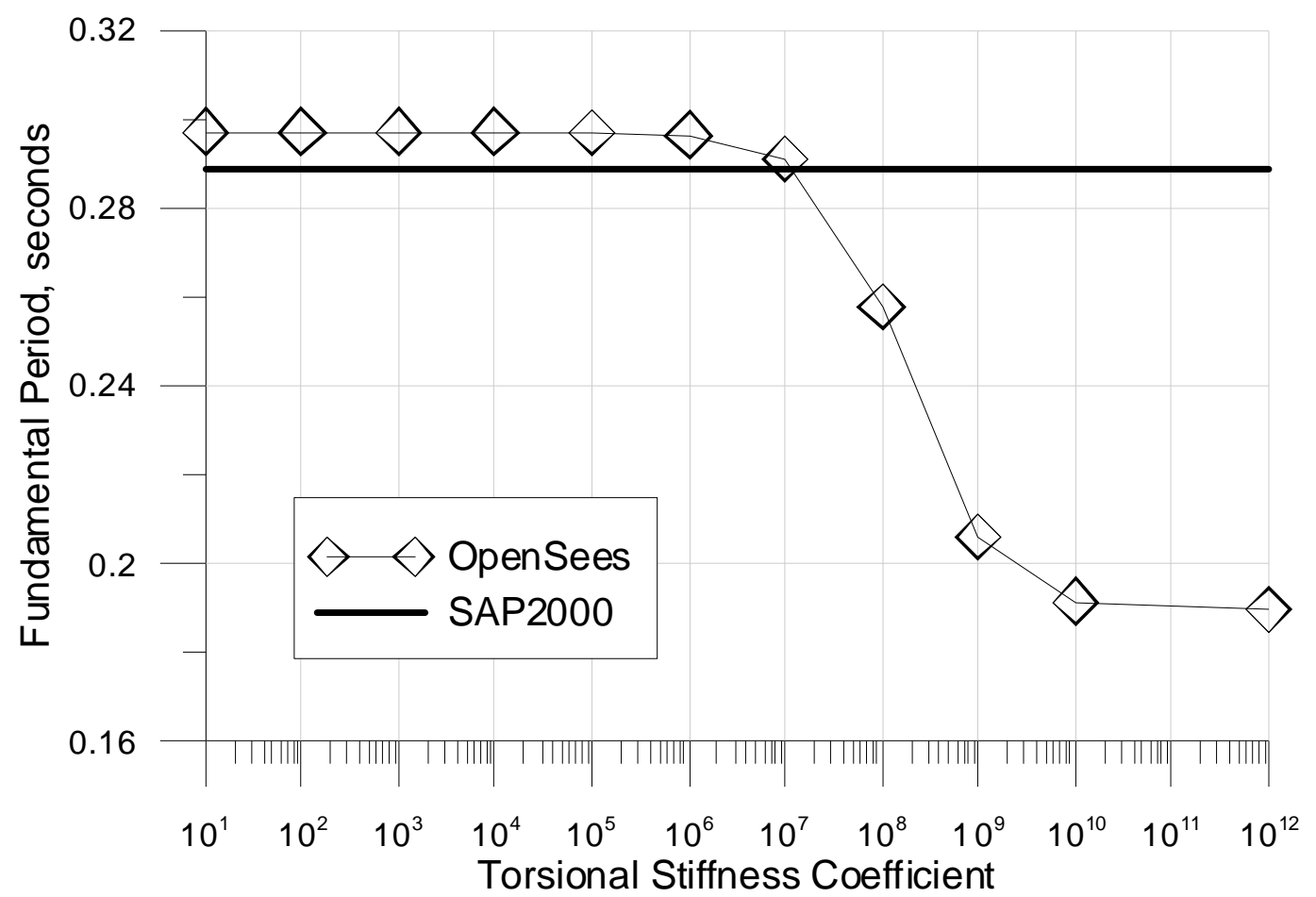

Figure 3.3: Fundamental period vs. torsional stiffness coefficient

A model identical to the OpenSees' simple single-story model was constructed in SAP2000 for comparison purposes. Both models were analyzed with identical seismic masses and reported similar translational periods along each of the plan dimensions. The similarities between the translational periods suggest reasonable results and congruency between the two models. The difference observed between the two models was in the rotational period. The solid line in Fig. 3.3 above shows the unrestricted rotational period of the SAP2000 model. As the torsional coefficient of the OpenSees model is reduced the rotational period converges to the expected 
value from the SAP2000 model. Fig. 3.3 above shows that the rotational period of the model converges with the SAP2000 prediction at a torsional stiffness coefficient of about 1.0e06.

As the original coefficient value of $1.0 \mathrm{e} 10$ is reduced, the building is able to develop the desired rotation and behave as expected. Both the orthogonal drift results and rotational periods converge to common values at a torsional stiffness coefficient of $1.0 \mathrm{e} 06$. Therefore, for the purposes of this project, a 3D model stiffness coefficient of $1.0 \mathrm{e} 06$ will be applied to each of the three Los Angeles models: three-story, nine-story, and twenty-story.

\subsection{Los Angeles Models}

\subsubsection{Three-Story Los Angeles, Post-Northridge Model}

As previously stated, for simplicity reasons only the perimeter moment frames are included in the OpenSees model of the Los Angeles three-story building. The placement of the moment resisting frames is shown in bold at the bottom of Figure A.1, in Appendix A. All of the gravity frames are neglected, including two of the corner columns for each floor level. The two neglected columns are the corner columns along column line "E." Nodes at each of the floor levels were placed in lieu of these columns to establish a symmetric rectangular perimeter for the rigid floor diaphragms. These nodes also help to provide a symmetric location to distribute the mass.

As in the Gupta et al. (1999) paper, the columns within each moment resisting frame are orientated such that bending about their strong axis occurs with the longitudinal direction of each particular frame. Within each frame there are two sets of columns: exterior and interior, which are described in Table 3.1. A generic detail of the column orientation can be found in Figure 3.1. All of the connections are full moment connections and the base of the first floor columns are considered to be fully restrained. 
Table 3.1: Three-story column and beam sizes

\begin{tabular}{|c|c|c|c|c|}
\hline \multirow{2}{*}{$\begin{array}{l}\text { Story/ } \\
\text { Floor }\end{array}$} & \multicolumn{2}{|c|}{ Columns } & \multirow{2}{*}{$\begin{array}{c}\text { Doubler } \\
\text { Plates (in) }\end{array}$} & \multirow[b]{2}{*}{ Girder } \\
\hline & Exterior & Interior & & \\
\hline $1 / 2$ & W14X257 & W14X311 & 0,0 & W30X116 \\
\hline $2 / 3$ & W14X257 & W14X311 & 0,0 & W30X116 \\
\hline 3/Roof & W14X257 & W14X311 & 0,0 & W24X62 \\
\hline
\end{tabular}

\subsubsection{Nine-Story Los Angeles, Post-Northridge Model}

As with the three-story model, only the moment frames were included in the nine-story model. The location of the moment frames are shown highlighted in bold at the bottom of Fig. A.1 in Appendix A. The moment frames of the nine-story model extend the full length of each plan dimension and are connected at each corner to one another, unlike the three-story model in which each of the moment frames were independent from each other. Consequently, with two perpendicular moment resisting frames framing together at each of the corners, the corner columns would be under biaxial bending. To avoid the biaxial bending, half of the beam moment connections framing into the corner columns have been released and act as pin connections. This is identified by the open circles at the corner columns at the bottom of Fig. A.1. To model this connection in OpenSees, “zeroLength" elements were used. These elements are defined by two different material models: one for the three degrees of translation and another for the three degrees of rotation. For the translational material model, an elastic model was used with a modulus of $29000 \mathrm{ksi}$, which allows for the direct transfer of shear. For the rotational material model, an elastic perfectly plastic model, ElasticPP, was used. Again the elastic modulus was $29000 \mathrm{ksi}$, but the strain at which plasticity is achieved is set to essentially zero. By setting the plastic strain to zero, the "zeroLength" element is unable to develop any significant moment capacity and, therefore, is unable to transfer any moments to the corner column.

The nine-story model also required that column cross-section size decrease throughout the height of the building. The column sizes and locations are listed in Table 3.2. The location of the splices 
is defined by Gupta et al. (1999) as six feet above the floor level. Six feet is essentially half the height of each inter-story column, 13 feet, and is intended to be located at the approximate inflection point between the floors. At each of the locations listed in Table 3.2 below $(2 / 3,4 / 5$, $6 / 7,8 / 9)$ an additional set of nodes was defined and the columns of different cross-sections were able to be spliced together.

Table 3.2: Nine-story column and beam sizes

\begin{tabular}{|c|c|c|c|c|}
\hline \multirow{2}{*}{$\begin{array}{l}\text { Story/ } \\
\text { Floor }\end{array}$} & \multicolumn{2}{|c|}{ Columns } & \multirow{2}{*}{$\begin{array}{c}\text { Doubler } \\
\text { Plates (in) }\end{array}$} & \multirow[b]{2}{*}{ Girder } \\
\hline & Exterior & Interior & & \\
\hline$-1 / 1$ & W14X370 & W14X500 & 0,0 & W36X150 \\
\hline $1 / 2$ & W14X370 & W14X500 & 0,0 & W36X150 \\
\hline $2 / 3$ & W14X370, W14X370 & W14X500, W14X455 & 0,0 & W36X150 \\
\hline $3 / 4$ & W14X370 & W14X455 & 0,0 & W33X141 \\
\hline $4 / 5$ & W14X370, W14X283 & W14X455, W14X370 & 0,0 & W33X141 \\
\hline $5 / 6$ & W14X283 & W14X370 & 0,0 & W33X141 \\
\hline $6 / 7$ & W14X283,W14X257 & W14X370, W14X283 & $0,1 / 2$ & W33X130 \\
\hline $7 / 8$ & W14X257 & W14X283 & 0,0 & W27X102 \\
\hline $8 / 9$ & W14X257, W14X233 & W14X283, W14X257 & $0,1 / 2$ & W27X94 \\
\hline 9/Roof & W14X233 & W14X257 & 0,0 & W24X62 \\
\hline
\end{tabular}

The columns in the moment frame are all orientated with their strong axis along the longitudinal direction of the frame. The bases of the basement columns are assumed to be pin connections and all of the nodes at the base of the first floor columns, ground level, are restrained against translation in all three directions but are free to rotate.

\subsubsection{0-Story Los Angeles, Post Northridge Model}

As previously done with the three-story and nine-story models, only the moment frames of the twenty-story model were included. The moment resisting frames of the twenty-story model extend the full length of each plan dimension and frame into the corner columns. For this model 
all of the connections are fully fixed, and the corner columns have box cross sections to accept the bi-axial bending.

Table 3.3: Twenty-story column and beam sizes

\begin{tabular}{|c|c|c|c|c|}
\hline \multirow{2}{*}{$\begin{array}{l}\text { Story/ } \\
\text { Floor }\end{array}$} & \multicolumn{2}{|c|}{$\begin{array}{l}\text { Columns } \\
\end{array}$} & \multirow{2}{*}{$\begin{array}{c}\text { Doubler } \\
\text { Plates (in) } \\
\end{array}$} & \multirow[b]{2}{*}{ Girder } \\
\hline & Exterior & Interior & & \\
\hline$-2 /-1$ & $15 \times 15 \times 2.00$ & W24X335 & 0,0 & W14X22 \\
\hline$-1 / 1$ & $15 \times 15 \times 2.00$ & W24X335 & 0,0 & W30X99 \\
\hline $1 / 2$ & $15 \times 15 \times 2.00$ & W24X335 & 0,0 & W30X99 \\
\hline $2 / 3$ & $15 \times 15 \times 2.00,15 \times 15 \times 1.25$ & W24X335, W24X335 & 0,0 & W30X99 \\
\hline $3 / 4$ & $15 \times 15 \times 1.25$ & W24X335 & 0,0 & W30X99 \\
\hline $4 / 5$ & $15 \times 15 \times 1.25$ & W24X335 & 0,0 & W30X99 \\
\hline $5 / 6$ & $15 \times 15 \times 1.25,15 \times 15 \times 1.00$ & W24X335, W24X279 & 0,0 & W30X108 \\
\hline $6 / 7$ & $15 \times 15 \times 1.00$ & W24X279 & 0,0 & W30X108 \\
\hline $7 / 8$ & $15 \times 15 \times 1.00$ & W24X279 & 0,0 & W30X108 \\
\hline $8 / 9$ & $15 \times 15 \times 1.00,15 \times 15 \times 1.00$ & W24X279, W24X279 & 0,0 & W30X108 \\
\hline $9 / 10$ & $15 \times 15 \times 1.00$ & W24X279 & 0,0 & W30X108 \\
\hline $10 / 11$ & $15 \times 15 \times 1.00$ & W24X279 & 0,0 & W30X108 \\
\hline $11 / 12$ & $15 \times 15 \times 1.00,15 \times 15 \times 1.00$ & W24X279, W24X229 & 0,0 & W30X99 \\
\hline $12 / 13$ & $15 \times 15 X 1.00$ & W24X229 & 0,0 & W30X99 \\
\hline $13 / 14$ & $15 \times 15 \times 1.00$ & W24X229 & 0,0 & W30X99 \\
\hline $14 / 15$ & $15 \times 15 \times 1.00,15 \times 15 \times 0.75$ & W24X229, W24X162 & $0,5 / 8$ & W30X99 \\
\hline $15 / 16$ & $15 \times 15 \times 0.75$ & W24X162 & $0,5 / 8$ & W30X99 \\
\hline $16 / 17$ & $15 \times 15 \times 0.75$ & W24X162 & $0,5 / 8$ & W30X99 \\
\hline $17 / 18$ & $15 \times 15 \times 0.75,15 \times 15 \times 0.75$ & W24X162, W24X117 & $0,5 / 8$ & W27X84 \\
\hline $18 / 19$ & $15 \times 15 \times 0.75$ & W24X117 & $0,5 / 8$ & W27X84 \\
\hline $19 / 20$ & $15 \times 15 \times 0.75,15 \times 15 \times 0.50$ & W24X117, W24X94 & $0,1 / 2$ & W24X62 \\
\hline 20/Roof & $15 \times 15 \times 0.50$ & W24X94 & 0,0 & W21X50 \\
\hline
\end{tabular}

All of the columns are orientated with their strong axis about the longitudinal direction of each frame. Additional nodes were also added at column splice locations (defined in Table 3.3) so that the column cross-sections could be accurately reduced throughout the height of the building. All of the element connections are full moment connections. The base of the bottom columns, second basement columns, are assumed to be pin connections and all of the nodes at the base of the first 
floor columns and first basement columns are restrained about translation in all three directions but are free to rotate.

\subsubsection{Seismic Model Assumptions}

For seismic upgrades in accordance with FEMA 267 some of the beams and girders were reinforced with doubler plates at column connections. These plates prevent premature failure due to panel zone effects and ensure ductile behavior of beam-to-column connections. Though these doubler plates do technically increase the stiffness of the individual beams and girders locally, they have a minimal effect on the global stiffness of the structure (Gupta, 1999, p. 346). Since the failure of the connections is not the concern of this research, and since their effect is negligible, the doubler plates are not explicitly considered in the models.

\subsection{Model Convergence}

In addition to modeling the buildings with the appropriate dimensions and cross-sections the model mesh has to be refined and checked for convergence. There are different types of elements that can be used in the numerical modeling, each one with its own limitations, and they also need to be checked for convergence.

\subsubsection{Number of Integration Points vs. Number of Elements}

OpenSees has two methods for refining the model mesh and obtaining more accurate results: increasing the number of integration points and increasing the number of elements. Increasing the number of integration points along the length of the elements is a simple code command to 
implement. With each consecutive increment of integration points the computational time remains relatively unaffected. However, increasing the number of elements requires intermediate nodes to be defined along the length of the column or beam. This increase in the number of nodes and the number of elements significantly increases the modeling time and, more importantly, the computational time. Both of these refining methods were tested on the simple single story model with force-based elements (FBE).

The single-story model was modified into four dimensionally identical models to evaluate the effects of the number of elements in the model. The first model had one single element spanning the entire column length; the second model had two elements per column length; the third had three elements; and the fourth had four. Each of these models was then further modified with a range of integration points, two through five to evaluate the effect of the number of integration points in the model. The fundamental period for each of these four structures and their four respective variations is listed in Table 3.4 below.

Table 3.4: Fundamental period of simple single-story model, seconds

\begin{tabular}{lllll}
\hline Number of & \multicolumn{4}{c}{ Number of Elements Along Column } \\
Integration Points & 1 & 2 & 3 & 4 \\
\hline 2 & 0.4910 & 0.4653 & 0.4604 & 0.4587 \\
3 & 0.3364 & 0.3364 & 0.3364 & 0.3364 \\
4 & 0.3364 & 0.3364 & 0.3364 & 0.3364 \\
5 & 0.3364 & 0.3364 & 0.3364 & 0.3364 \\
\hline
\end{tabular}

Each of the models was then excited by the Kocaeli Turkey, Duzce station, pair of bi-directional ground motions and the maximum story drifts are recorded in Table 3.5 below. 
Table 3.5: Maximum story drift of simple single-story model

\begin{tabular}{lllll}
\hline Number of & \multicolumn{4}{c}{ Number of Elements Along Column } \\
Integration Points & 1 & 2 & 3 & 4 \\
\hline 2 & $3.64 \%$ & $4.32 \%$ & $4.30 \%$ & $4.29 \%$ \\
3 & $2.32 \%$ & $2.32 \%$ & $2.32 \%$ & $2.32 \%$ \\
4 & $2.28 \%$ & $2.28 \%$ & $2.28 \%$ & $2.28 \%$ \\
5 & $2.28 \%$ & $2.28 \%$ & $2.28 \%$ & $2.28 \%$ \\
\hline
\end{tabular}

Each set of data in Tables 3.4 and 3.5 shows that regardless of the number of elements along the length of the column, the models are able to converge with four to five integration points. The integration technique being used in these models is Gauss-Lobatto Integration. According to Scott (2011), Gauss-Lobatto Integration is common for members with maximum bending moments at the ends of the elements. This technique applies integration points at both ends, for a minimum of two points, and then places the rest along the length of the element. For the analysis in this project all of the loads are applied at the floor level, and the largest moments are assumed to occur at the floor level, at the beam ends and the column ends. Therefore, the number of elements has a nearly negligible effect. For these reasons, the three Los Angeles models will be modeled with single column elements and the number of integration points for each building will be tested for convergence. This will decrease the complexity of the models and greatly reduce the computational time.

\subsubsection{Force-Based Elements versus Displacement-Based Elements}

To determine which element types to use in the numerical model, a comparison was done between force-based elements and displacement-based elements. To compare the OpenSees' "BeamColumn" elements, two identical three-story models were created: one with displacementbased elements, DBE, and the other with force-based elements, FBE, both of which were excited with the same pair of bidirectional ground motions, Kocæli Turkey, Duzce station. For this 
evaluation, the Los Angeles three-story model was chosen. One model was built with the displacement-based elements, "dispBeamColumn", and the other with force-based elements, "nonlinearBeamColumn." Both elements have distributed plasticity. To compare the different element types, the relative displacements for each floor were analyzed in both the primary and minor directions, where primary is defined as the direction parallel with the stronger ground motion and minor with the weaker ground motion. The weaker ground motion is defined as the component with the smaller spectral acceleration at the fundamental period of the building. The primary ground acceleration history along the primary direction is shown in Fig. 3.4.

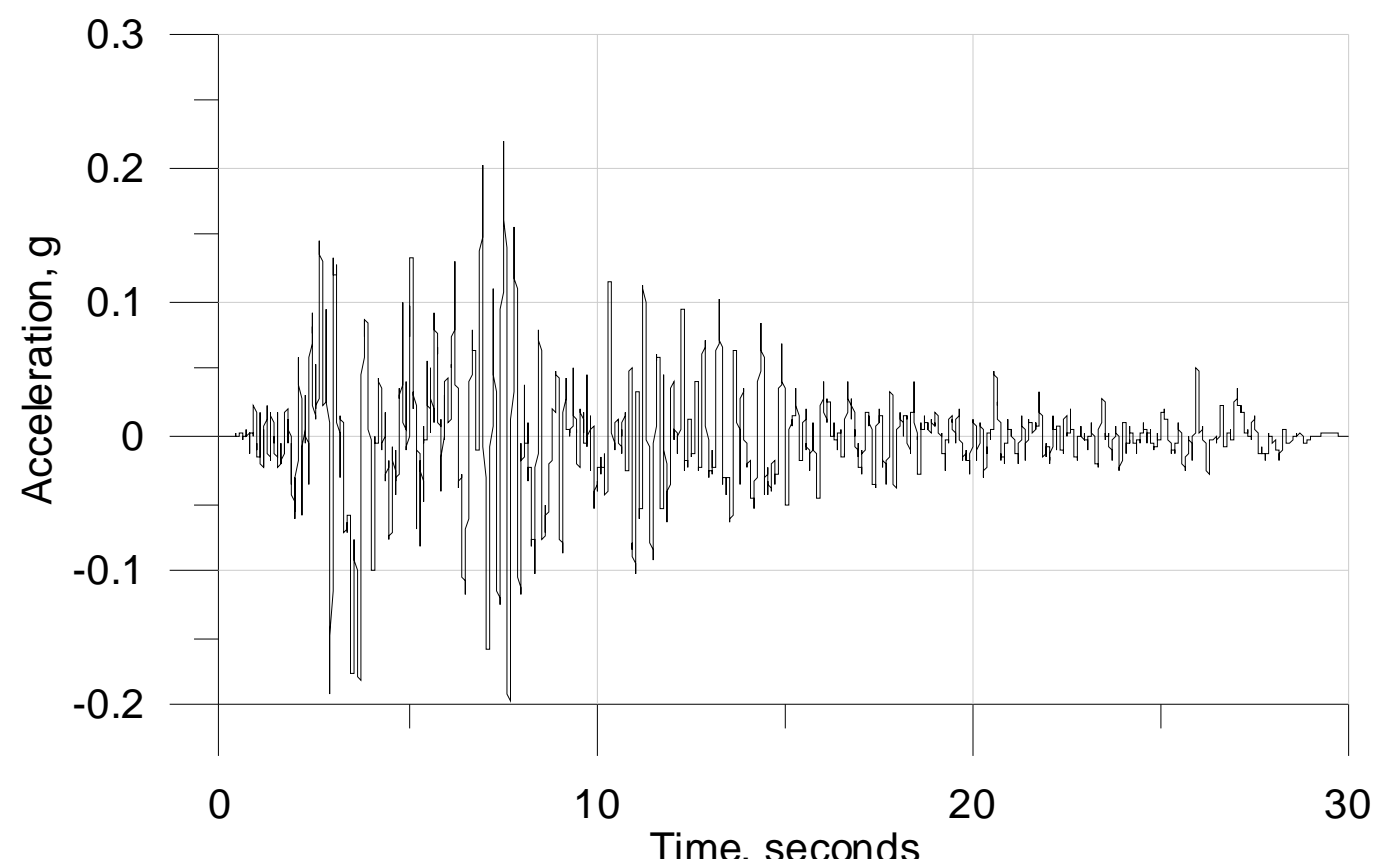

Figure 3.4: Kocæli Turkey primary component acceleration time history

The time history plot of the first floor drifts in the primary direction is shown in Fig. 3.5. The two curves - displacement-based elements and force-based elements - are shown on top of each other. The displacements of the force-based elements are mostly on the conservative side. After significant yielding of the first floor members, at around four seconds, the displacement-based model reports drift values $0.6-0.9 \%$ less than the force-based model. This conservatism persists 
during the high amplitude period of excitation, and as the excitation decreases the two models converge back to reporting similar results.

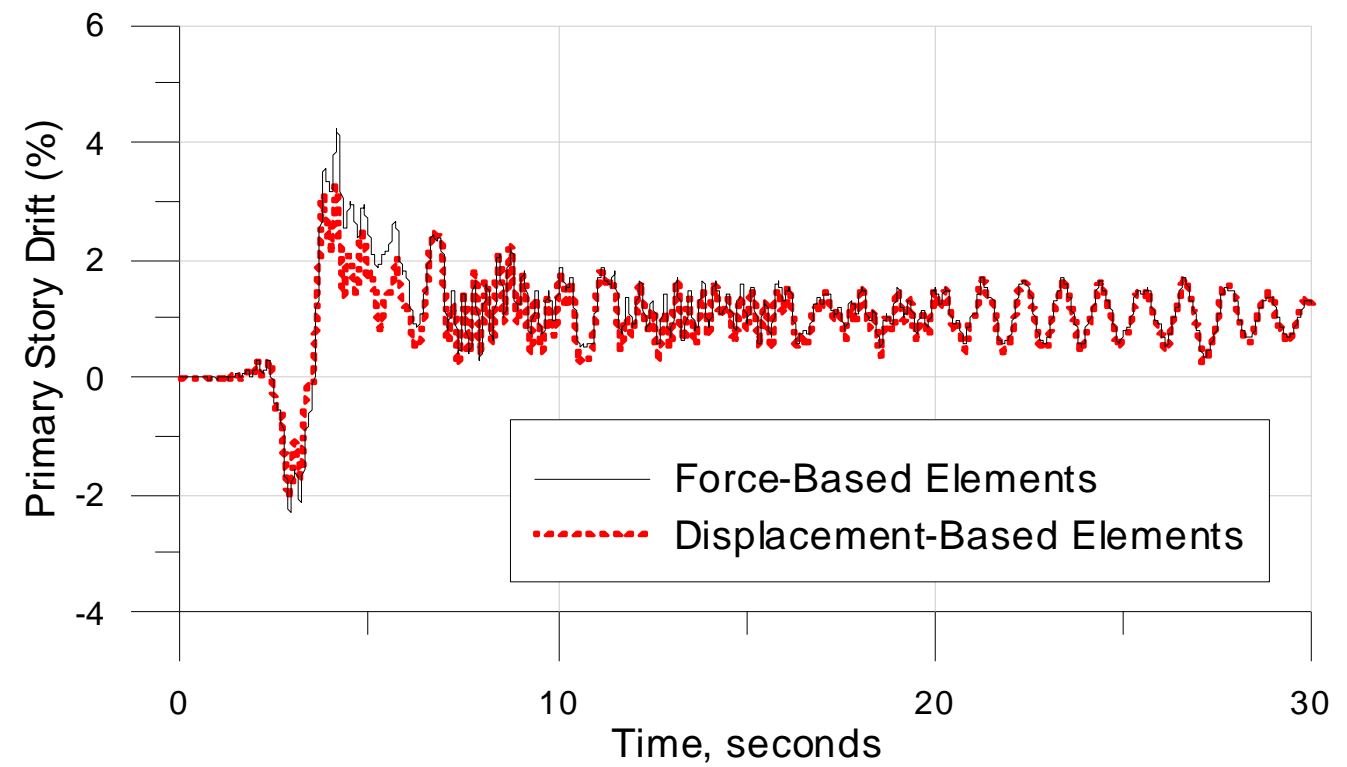

Figure 3.5: Primary direction FBE vs. DBE drift comparison

The absolute difference in drift values for the two models is shown in Fig. 3.6. The positive values indicate the amount of conservatism of the force-based elements over the displacementbased elements.

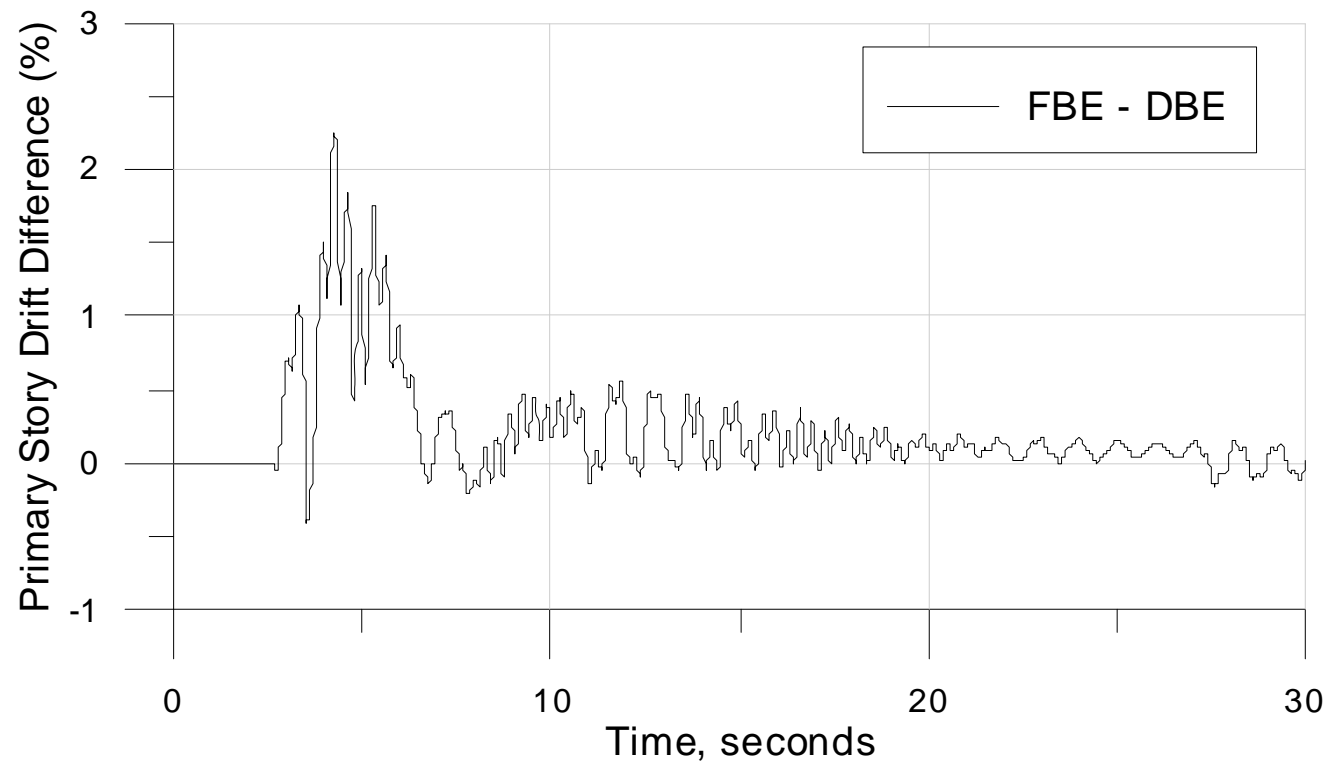

Figure 3.6: FBE minus DBE drifts along primary direction 
The maximum absolute drift values are listed below in Table 3.6. The top three rows of the table report the maximum drift values for both models at each of the three floor levels. These maximum drifts do not necessarily occur at the same time step. The force-based elements are 0.6$0.9 \%$ more conservative than the displacement-based elements. The bottom row of the table reports the maximum drift difference between models at any individual time step. These differences range from $1.2-1.5 \%$. Therefore, at any instance of the time history analysis the two different models' element types could report significantly different values, while over the entire time history the absolute maximum drifts are closer in value.

Table 3.6: DBE vs FBE comparison along primary direction

\begin{tabular}{lllll}
\hline & & Floor 1 & Floor 2 & Floor 3 \\
\hline Max Drift & Displacement-Based & $3.03 \%$ & $4.13 \%$ & $5.74 \%$ \\
& $\begin{array}{l}\text { Force-Based } \\
\text { Difference of }\end{array}$ & $3.93 \%$ & $4.85 \%$ & $6.37 \%$ \\
& Maximums & $0.90 \%$ & $0.72 \%$ & $0.63 \%$ \\
\hline & & & \\
\hline & Max Difference & $1.34 \%$ & $1.14 \%$ & $1.52 \%$ \\
\hline
\end{tabular}

The next set of figures is for the drifts in the minor direction, drift about the weaker direction of excitation or perpendicular to the primary direction. Fig. 3.7 shows that, once again, the difference between the two models is seen at periods of high amplitude ground excitation, and the two curves converge back as the excitation decreases. Unlike in the case of the primary direction, the drifts in the minor direction do not show the same consistent conservatism of the force-based elements. 


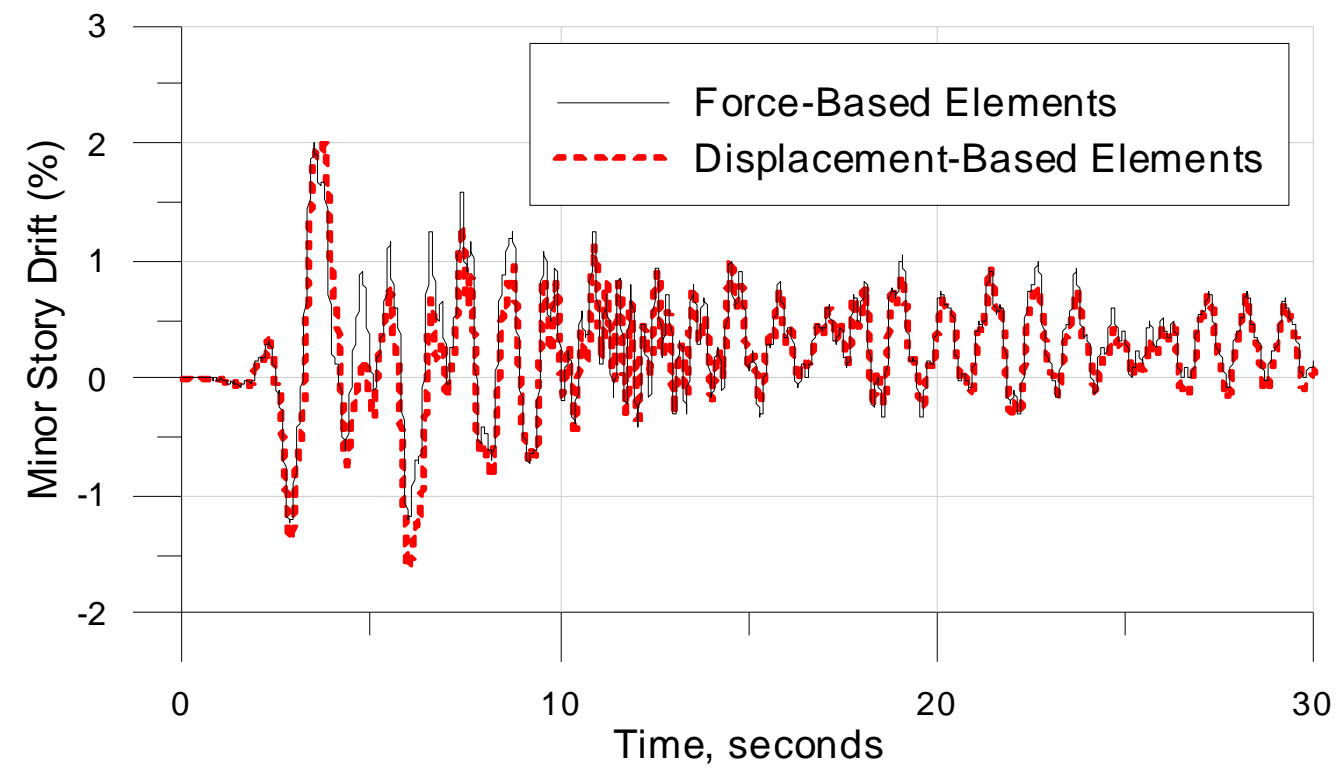

Figure 3.7: Minor direction FBE vs. DBE drift comparison

Fig. 3.8 shows the absolute difference between the two values. Unlike the primary direction in which the absolute plot was mainly positive showing the constant conservatism of the force-based elements, this plot wavers between positive and negative.

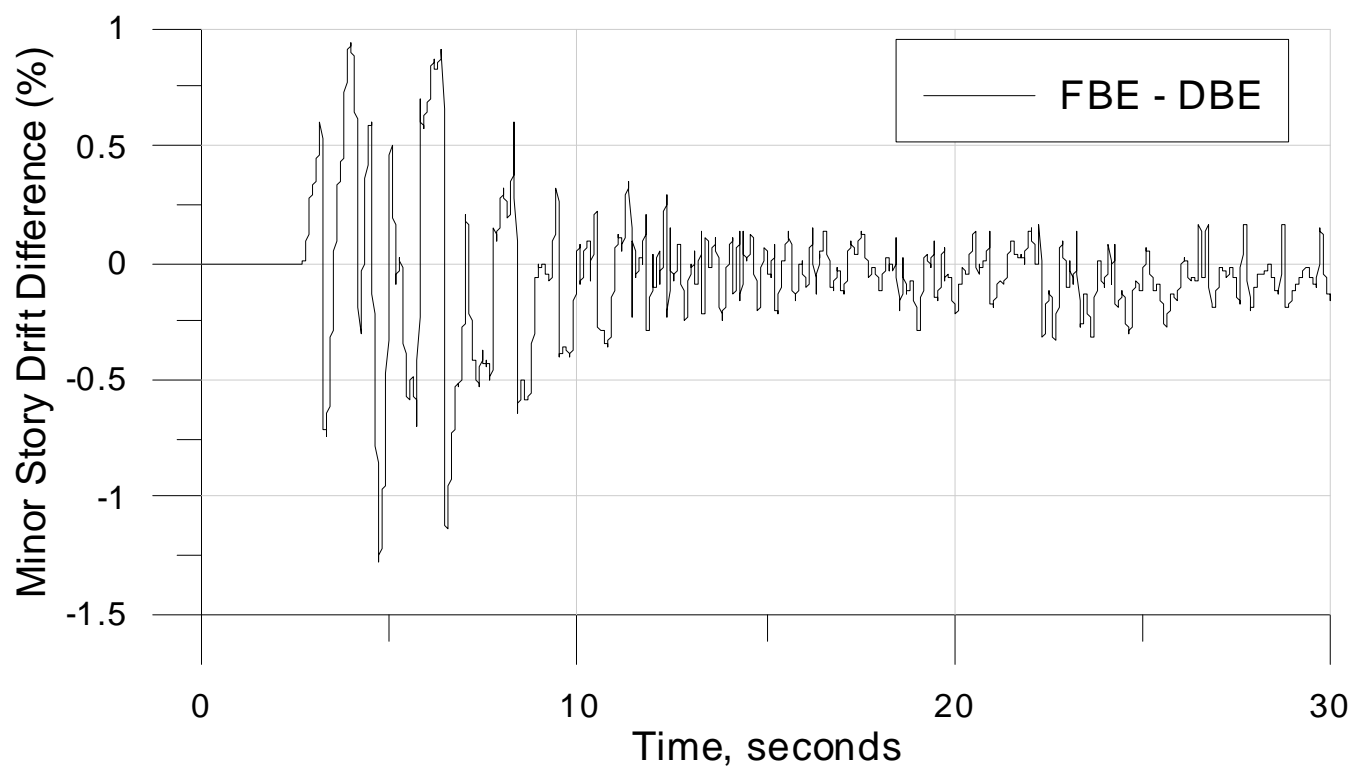

Figure 3.8: FBE minus DBE drifts along minor direction

From Table 3.7 below, the absolute maximum drift values for the two models are much closer together than for the primary direction. Also, unlike the primary direction, the displacement- 
based element model reported the more conservative values, although the difference is negligible. Once again the maximum differences are much greater showing the variability between the models at any given time step.

Table 3.7: DBE vs. FBE comparison along minor direction

\begin{tabular}{lllll}
\hline & & Floor 1 & Floor 2 & Floor 3 \\
\hline Max Drift & Displacement Based & $1.89 \%$ & $2.74 \%$ & $3.39 \%$ \\
& $\begin{array}{l}\text { Force Based } \\
\text { Dalues } \\
\text { Maxference of }\end{array}$ & $1.85 \%$ & $2.65 \%$ & $3.27 \%$ \\
& & $-0.04 \%$ & $-0.10 \%$ & $-0.13 \%$ \\
\hline & & & \\
\hline
\end{tabular}

The differences observed between the primary and minor directions are attributed to the amount of deformation that occurs along each direction. Along the primary direction, the direction of greater ground excitation, greater drifts and nonlinear behavior are observed. These drifts are on average twice that of the minor direction drifts. According to Correia et al. (2008), both forcebased and displacement-based elements can handle nonlinear behavior, but each one has its own limitations. The displacement-based elements are computationally inexpensive, but require a greater number of elements to converge. The force-based elements are more computationally expensive but are able to converge with an increase of integration points along the member. Since the columns and beams are modeled as single elements spanning between floors and bays, respectively, force-based elements are used for each of the three Los Angeles models and the model mesh is refined through an increase in integration points. 


\subsection{Floor Load Parameters: 3-, 9-, and 20-Story Models}

For the parametric analysis the load intensity and acting locations for each individual floor within the models needs to be determined. The nominal load intensities are taken from Gupta et al. (1999) and the loading parameters, intensity variation and acting location, are based on the research done by De-la-Colina et al. (2011).

\subsubsection{Determination of Floor Loads}

The floor loads used for each building, which were determined based on Gupta et al. (1999), is provided in Table 3.8 .

Table 3.8: Building loads

\begin{tabular}{lll}
\hline Stanford Loads & & \\
\hline DL & 86 & psf \\
DL, roof & 83 & psf \\
DL, penthouse & 116 & psf \\
LL & 20 & psf \\
\hline
\end{tabular}

The left portion of Table 3.9, under the "Stanford" heading, has the building dimensions and seismic mass as described by Gupta et al. (1999) for each of the three Los Angeles office buildings. The total load, psf, has been back calculated from the seismic mass and used for comparison purposes to the hand calculated values listed on the right portion of the table under the "Hand Calculated" column heading. 
Table 3.9: Mass comparison: Stanford vs. hand calculated

\begin{tabular}{|c|c|c|c|c|c|c|c|c|c|}
\hline & & \multicolumn{4}{|c|}{ Stanford } & \multicolumn{4}{|c|}{ Hand Calculated } \\
\hline & & $\begin{array}{l}\text { Dime } \\
\mathrm{b}, \mathrm{ft}\end{array}$ & $\begin{array}{r}\text { sions } \\
\mathrm{w}, \mathrm{ft} \\
\end{array}$ & $\begin{array}{l}\text { Total } \\
\text { psf }\end{array}$ & $\begin{array}{l}\text { Seismic } \\
\text { Mass } \\
\operatorname{kips}^{*} \sec ^{2} / \mathrm{ft}\end{array}$ & $\begin{array}{l}\text { DL }_{\text {effective }} \\
\text { psf }\end{array}$ & $\begin{array}{l}\text { LL }_{\text {effective }} \\
\text { psf }\end{array}$ & $\begin{array}{l}\text { Total } \\
\text { psf }\end{array}$ & $\begin{array}{l}\text { Seismic } \\
\text { Mass } \\
\mathrm{kips}^{*} \mathrm{sec}^{2} / \mathrm{ft}\end{array}$ \\
\hline \multirow{2}{*}{$\begin{array}{l}3 \\
\text { Story }\end{array}$} & Roof & 120 & 180 & 105.6 & 70.9 & 92.7 & 10 & 102.7 & 68.93 \\
\hline & Floors 2-3 & 120 & 180 & 97.6 & 65.53 & 86 & 10 & 96 & 64.45 \\
\hline \multirow{3}{*}{$\begin{array}{l}9 \\
\text { Story }\end{array}$} & Roof & 150 & 150 & 104.5 & 73.1 & 92.3 & 10 & 102.3 & 71.53 \\
\hline & Floor 3-9 & 150 & 150 & 97 & 67.86 & 86 & 10 & 96 & 67.13 \\
\hline & Floor 2 & 150 & 150 & 98.7 & 69.04 & 86 & 10 & 96 & 67.13 \\
\hline \multirow{3}{*}{$\begin{array}{l}20 \\
\text { Story }\end{array}$} & Roof & 100 & 120 & 107.4 & 40.06 & 90.7 & 10 & 100.7 & 37.57 \\
\hline & Floor 3-20 & 100 & 120 & 101.2 & 37.76 & 86 & 10 & 96 & 35.81 \\
\hline & Floor 2 & 100 & 120 & 103.6 & 38.63 & 86 & 10 & 96 & 35.81 \\
\hline
\end{tabular}

The difference seen in seismic masses between the roof level and the floor levels below is attributed to the increased penthouse loads and the difference in tributary wall area at the top level. The difference between the seismic masses for floor 2 and the floors above is attributed to the difference in tributary wall area because the bottom floors have a height of 18 feet while the floors above are all 13 feet. The hand calculated seismic masses only consider the floor areas and neglect the tributary wall area. As can be seen above, the dead loads for all the floors below the roof level are 86 psf as suggested in Gupta et al. (1999). The increase of dead loads at the roof level is due to the greater penthouse loads. Each floor has an identical live load of $10 \mathrm{psf}$, which is the effective seismic live load according to ASCE 7-10 (2010). These hand calculated total loads are comparable to those provided in Gupta et al. (1999).

The discrepancy between the hand calculated seismic masses and those masses provided by Gupta et al. (1999) is partially due to the exterior wall cladding that was not included, which is why all of the calculated masses are less than the values in the Stanford research. The focus of this research is on the effect of load distribution, and the parameters being used do not include the load distribution of exterior walls and cladding. Therefore, the contribution of the exterior walls has been left out and the seismic masses are only slightly reduced because of it. 


\subsubsection{Monte Carlo Simulation: Effective Load Intensity and Location}

The nominal dead and live loads that will be used here were provided by the Gupta et al. (1999), which, however, does not provide a suggested accidental eccentricity or distribution for these loads. A survey of dead and live load distributions for several office buildings in Mexico City is summarized in a paper by De-la-Colina et al. (2011) which lists values for the coefficient of variation for both dead and live load intensities and positions. Although this survey was done in Mexico City, the buildings surveyed have dead to live load ratios similar to the Los Angeles office buildings, and, since both are office buildings, the parameters are applicable to the Los Angeles models.

For analysis purposes the dead and live loads needed to be combined into one effective load with one distribution of intensity and position. De-la-Colina et al. (2011) stated that the dead load intensity is best represented with a normal distribution while the live load intensity is a better fit with a gamma distribution. The positions of each of these loads, dead and live, are represented with a normal distribution. In lieu of a closed form solution to this problem, the present research project used a Monte Carlo Simulation to combine both the dead and live load properties and to determine the mean and standard deviations of the effective load intensity and position.

\section{Monte Carlo Simulation:}

The dead and live loads determined from the Gupta et al. (1999) paper and the properties found from the De-la-Colina et al. (2011) paper were combined according to equations 3.1 and 3.2 below:

$$
\begin{aligned}
& \text { Position }_{X_{\text {Effective }}}=\left[\frac{\text { Intensity }_{\text {Live }} \times \text { Position }_{X_{\text {Live }}}+\text { Intensity }_{\text {Dead }} \times \text { Position }_{X_{\text {Dead }}}}{\text { Intensity }_{\text {Live }}+\text { Intensity }_{\text {Dead }}}\right] \\
& \text { Intensity }_{\text {Effective }}=\left[\text { Intensity }_{\text {Live }}+\text { Intensity }_{\text {Dead }}\right]
\end{aligned}
$$


The simulation was run until the position and intensity both converged to constant values. Below in Fig. 3.9, the mean effective load intensity for the lower floors can be seen converging to 96 psf, which is as expected from the total value listed in Table 3.9 above.

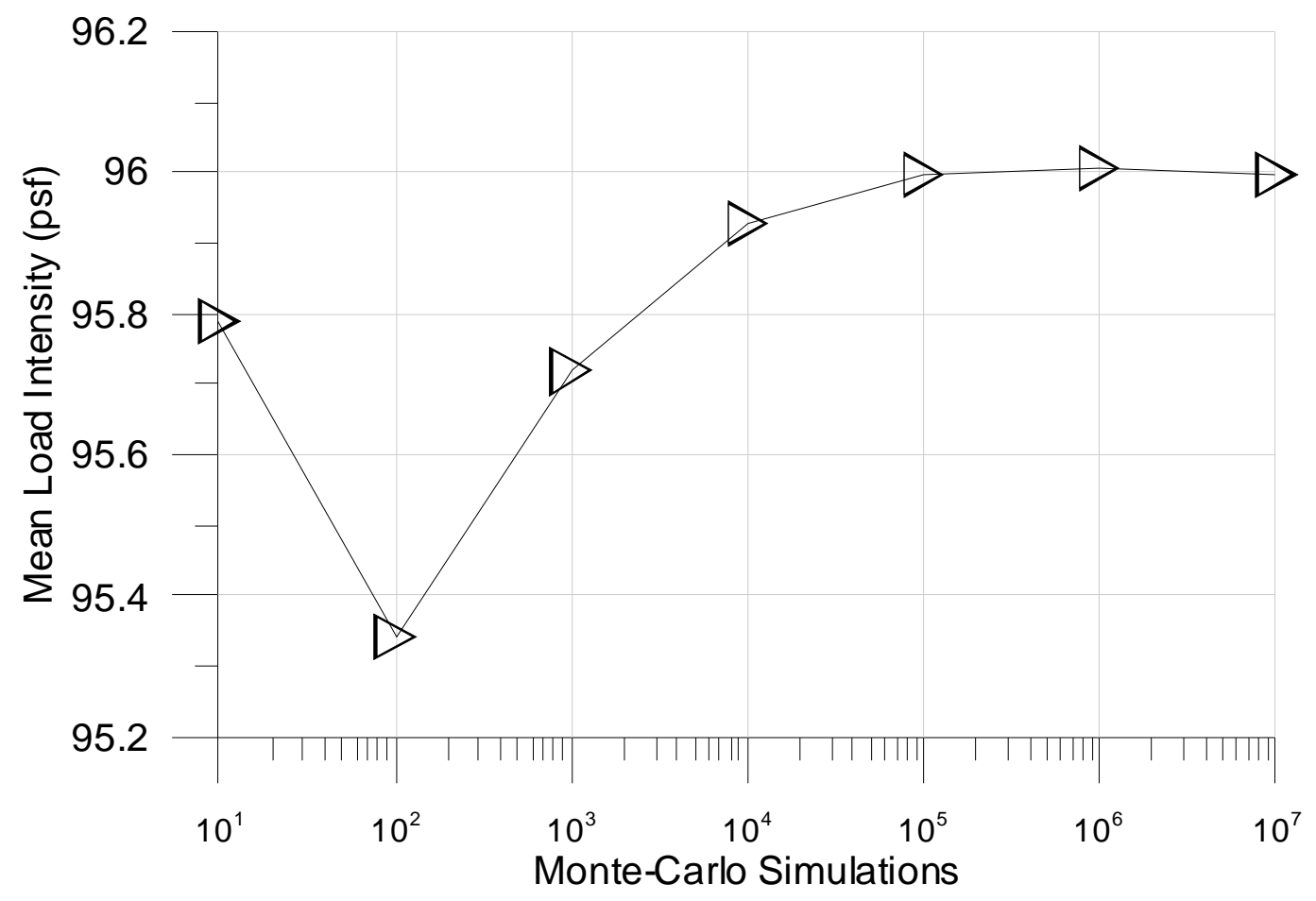

Figure 3.9: Convergence of effective mean load intensity, lower floors

As shown in Fig 3.10, the standard deviation of the effective load also converges to a constant value. This simulation process was repeated three more times for the effective roof loads and positioning for each of the three Los Angeles office buildings. 


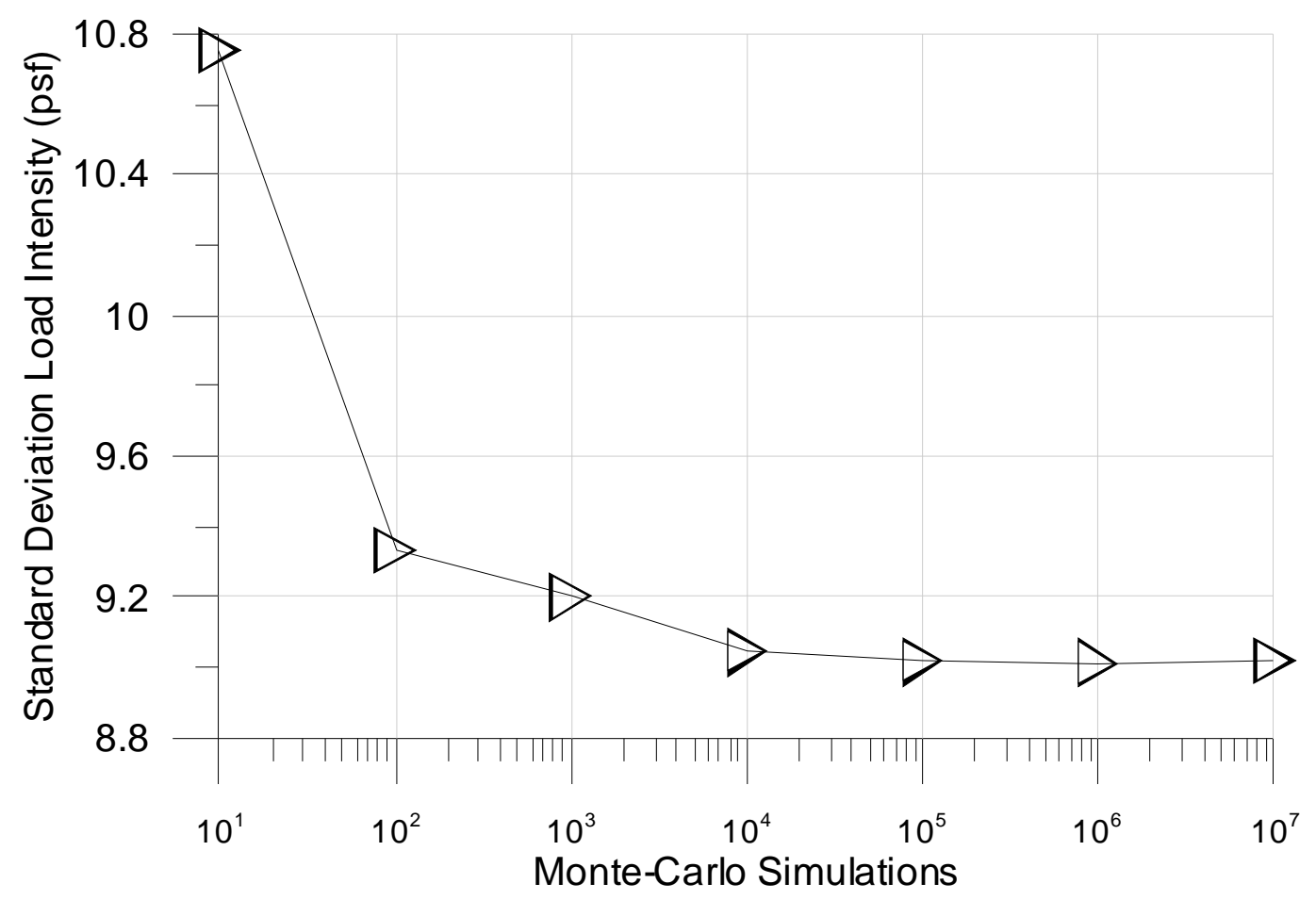

Figure 3.10: Convergence of effective load intensity standard deviation, lower floors

Table 3.10 lists the individual dead and live load parameters that went into the Monte Carlo Simulation and the results of that simulation. The top two sections of the table, Dead and Live Load Parameters, list the parameters that are input into the Monte Carlo Simulation. The "Position_X" and "Position_Y" describes the location of the acting dead and live loads along both of the buildings plan dimensions, $\mathrm{X}$ and $\mathrm{Y}$ respectively. All of the acting positions have a mean value of 0.5 , this corresponds to the position along the plan dimension, which results in the dead and live loads having a mean location at the geometric center of each floor level. The bottom portion of the table, Effective Load Parameters, list the results of the Monte Carlo Simulation. As expected, the effective load is a sum of the mean dead and live loads. Since both have the same mean eccentricity, the resultant mean eccentricity also remains the same. The main results from this set of simulations are the standard deviations of the effective load intensity and positioning of the effective load. 
Table 3.10: Intermediate floors for 3-,9-, and 20-story parameters

\begin{tabular}{llll}
\hline & & Mean, $\mu$ & Standard Deviation, $\sigma$ \\
\hline \multirow{2}{*}{ Dead Load Parameters } & Position_X_Dead & 0.5 & 0.025 \\
& Position_Y_Dead & 0.5 & 0.025 \\
& Load_Intensity_Dead (psf) & 86 & 8.6 \\
\hline \multirow{2}{*}{ Live Load Parameters } & Position_X_Live & 0.5 & 0.037 \\
& Position_Y_Live & 0.5 & 0.037 \\
& Load_Intensity_Live (psf) & 10 & 2.72 \\
\hline Effective Load & Position_X_Effective & 0.5 & 0.0228 \\
Parameters & Position_Y_Effective & 0.5 & 0.0228 \\
& Load_Intensity_Effective (psf) & 95.99 & 9.0193 \\
\hline
\end{tabular}

Tables A.2, A.3, and A.4 in Appendix A, have the effective load and distribution values for the roof level for each of the three models, respectively. All of these effective load values will be applied to the respective buildings in the parametric study.

\subsubsection{Seismic Mass Assignment}

To establish the desired seismic mass eccentricities the masses are assigned to the four corner nodes at each floor level. For the three-story building where not all of the moment frames extend to the corners of the building, special nodes were added to each floor at these corner locations. These nodes were fixed to the rigid floor diaphragm as well as against displacement in the vertical direction. For the other two corners in the three-story model, as well as all those in the nine-story and twenty-story models, the masses are simply assigned at the column joints at each floor level. To establish the eccentricity in each plan dimension, the proportion of the total seismic floor mass is adjusted between the four nodes, with the sum of all four nodes equal to the total seismic mass at each floor.

Besides the seismic mass, no other loads are assigned to the moment frames. The gravity frames are assumed to take most of the vertical forces, and since the moment frames are located on the 
exteriors of the buildings, the amount of gravity load in their tributary area is minimal. Therefore, no efforts were made to represent the gravity loads on the moment frames for the dynamic analyses and accordingly the P-Delta effect was not considered in this research. 


\section{Chapter 4: Model Validation:}

The models have been designed in OpenSees and many simplifications and assumptions were made. The types of elements used and the number of integration points for each element were compared and determined. Now each of the models needs to be validated to ensure that they are designed correctly and will produce reliable results.

\subsection{Translational Period Comparison}

The OpenSees models were modeled using the exact steel cross-sections and dimensions provided in Gupta et al. (1999) and listed in Tables 3.1-3.3. To validate the OpenSees models, the translational periods provided by Gupta et al. (1999) were compared with the corresponding periods calculated for the OpenSees models. Since the three models are either symmetric or nearly symmetric each one was chosen to be idealized as single frame two-dimensional models in the Stanford analysis. They were all analyzed about one plan direction defined as North-South (detailed in Figure A.1 of Appendix A) using one moment resisting frame with half of the seismic loads attributed to it. With this analysis the authors reported the periods of the first three translational modes of the moment frame along the stiff, longitudinal direction. All of the gravity frames are neglected and do not contribute to the stiffness of the models. The adjacent moment frames that are perpendicular to the frame of interest are also neglected and do not contribute to the overall stiffness of the models.

The OpenSees models, like the Stanford two-dimensional models, also neglect the gravity frames. However, unlike the Stanford models, the OpenSees models are all three-dimensional. With the three-dimensional analysis, the perpendicular moment frames do contribute to the stiffness of the model. The increase in the stiffness is minimal and is only expected to slightly decrease the 
translational periods of the OpenSees' models. For comparison purposes the translational periods of the OpenSees models were evaluated with the same seismic floor masses as defined in Gupta et al. (1999), as opposed to the seismic masses calculated in section 3.5.1. The seismic mass was evenly distributed to the four corner nodes of each floor. To analyze the periods of these models, each of the rigid floor diaphragms were restrained to allow only one degree of freedom: either translation along the $\mathrm{x}$-axis, translation along the $\mathrm{z}$-axis, or rotation about the $\mathrm{y}$-axis. The first three modes for each of these conditions were analyzed. The OpenSees models are orientated such that the x-axis corresponds with the North-South direction of the Stanford models. From this point forward the North-South direction of the Stanford models will be referred to as the x-axis or x-direction.

\subsubsection{Validation of Three-Story Model}

In Table 4.1, the first three translational periods along the x-axis of the Stanford three-story model are compared against the first three translational periods along the $\mathrm{x}$-axis of the OpenSees three-story model.

Table 4.1: Three-Story period comparison, seconds

\begin{tabular}{lllllll}
\hline & & Stanford & & \multicolumn{3}{l}{ OpenSees } \\
\cline { 5 - 7 } & & $\mathrm{X}$ & & $\mathrm{X}$ & $\mathrm{Z}$ & $\mathrm{Y}$, rotational \\
\hline Mode & 1 & 1.02 & & 1.016 & 1.016 & 1.038 \\
& 2 & 0.30 & & 0.311 & 0.311 & 0.331 \\
& 3 & 0.14 & & 0.151 & 0.151 & 0.168 \\
\hline
\end{tabular}

Here, the periods in the Stanford ' $\mathrm{X}$ ' column are nearly identical to the corresponding values in the OpenSees ' $\mathrm{X}$ ' column. To ensure the validity of the OpenSees' period values, an eigenvector analysis was performed. The mode shapes, translational and rotational, for each of the first three modes are plotted in Figure A.2 in Appendix A. Each mode shape is labeled with a letter and a 
number: the letter " $T$ " corresponds to translational and the letter " $R$ " corresponds to rotational. The numbers correspond to the first three modes, and the mode shapes confirm that each period value has been appropriately assigned. Therefore, the period analysis shows good correlation to the Stanford model at each of the first three modes and serves to validate the OpenSees' Los Angeles three-story model.

\subsubsection{Validation of Nine-Story Model}

A similar result comparison was made for the nine-story model. In Table 4.2, the first three translational periods along the $\mathrm{x}$-axis of the Stanford nine-story model are compared against the first three translational periods along the $\mathrm{x}$-axis of the OpenSees nine-story model.

Table 4.2: Nine-Story period comparison, seconds

\begin{tabular}{lllllll}
\hline & & \multicolumn{2}{l}{ Stanford } & & \multicolumn{3}{l}{ OpenSees } \\
\cline { 5 - 6 } & & $\mathrm{X}$ & & $\mathrm{X}$ & $\mathrm{Z}$ & $\mathrm{Y}$, rotational \\
\hline Mode & 1 & 2.21 & & 2.318 & 2.317 & 2.331 \\
& 2 & 0.82 & & 0.867 & 0.865 & 0.875 \\
& 3 & 0.46 & & 0.498 & 0.495 & 0.506 \\
\hline
\end{tabular}

Here, the translational periods of the OpenSees' nine-story building do not match up to the Stanford periods as nicely as they do with the three-story model. The mode shapes for the ninestory model are plotted in Fig. A.3 in Appendix A and ensure that the values have been assigned appropriately. The differences between the Stanford and OpenSees models in how they were modeled, as well as the software used to analyze them, may contribute to the differences seen between the Stanford and OpenSees' translational periods along the x-axis. The Stanford analysis was performed in 1999 and, due to a lack of analytical computer programs and computational power at the time, simplified building models were used. In addition to those differences, the modeling of the corner beam-column pin connections may also contribute to the differences seen 
between the two models. Finally, the differences between the Stanford analysis program, DRAIN-2DX, and the OpenSees analysis may contribute to inherent differences. With the increased complexity of the nine-story model over the three-story model the inherent differences in the software may also be attributing to the differences seen, but the difference between the periods is considered minor and the period analysis serves to validate the OpenSees' Los Angeles nine-story model.

\subsubsection{Validation of Twenty-Story Model}

The first three periods for the twenty-story are compared in Table 4.3.

Table 4.3: Twenty-Story period comparison, seconds

\begin{tabular}{lllllll}
\hline & \multicolumn{3}{l}{ Stanford } & & \multicolumn{3}{l}{ OpenSees } \\
\cline { 5 - 7 } & & $\mathrm{X}$ & & $\mathrm{X}$ & $\mathrm{Z}$ & $\mathrm{Y}$, rotational \\
\hline Mode & 1 & 3.65 & & 3.516 & 3.168 & 3.226 \\
& 2 & 1.26 & & 1.253 & 1.132 & 1.158 \\
& 3 & 0.72 & & 0.735 & 0.668 & 0.700 \\
\hline
\end{tabular}

Again, the translational periods of the twenty-story model do not match up as nicely to the Stanford periods as they do for the three-story model. The mode shapes for the twenty-story model are plotted in Fig. A.4 of Appendix A and ensure that the period values have been assigned appropriately. The differences observed are considered minor and the period analysis serves to validate the OpenSees' Los Angeles twenty-story model.

\subsection{Additional Period Analyses}

In addition to the first three periods along the $\mathrm{x}$-axis, the first three periods along the $\mathrm{z}$-axis and about the y-axis are also reported. As expected for the three-story and nine-story buildings, the 
translational periods along each of the plan dimensions are equivalent because the moment frames are identical on all four sides. However, the twenty-story building is not symmetric and has an additional moment resisting bay along the z-axis. This increase in stiffness along the z-axis is seen in the shorter translational periods along the z-axis.

Since all of the buildings are either symmetric or nearly symmetric with exterior moment frames, the rotational periods of each model match up with the respective translational periods. This coupling of the translational and rotational periods is expected to increase the rotational response. According a study done by Tso et al. (1980), the rotational response is greatly amplified even at small mass eccentricities when the translational to rotational period ratio is equal to one.

\subsection{Pushover Analysis}

In addition to the period and mode shape analysis, a static pushover analysis was performed on each of the buildings to ensure that they all behaved as expected. The story forces applied were based on a triangular load distribution with the assumed effective seismic masses from Chapter 3. Each model was pushed over to a roof displacement of ten percent of the building height. Fig. 4.1 below shows the inter-story displacements/drifts versus the respective column shear for the Los Angeles three-story model. Each of the story curves has an initial linear portion at lower displacements/drifts as expected. Each of the floors also transition to nonlinear behavior at the same displacement/drift of around two inches. The floors are expected to behave similarly and experience nonlinearity at similar displacements/drifts since the column cross-sections are identical at each floor level, and the beam cross-sections are nearly identical at each floor. The increased slope and shorter total displacement of the first floor-represented by the top curve- is due to the column constraints of the bottom floor. The bottom floor columns are fully fixed at the 
base and, therefore, exhibit the stiffer behavior seen below. The second and third-story column boundaries are able to rotate and consequently exhibit a less stiff behavior.

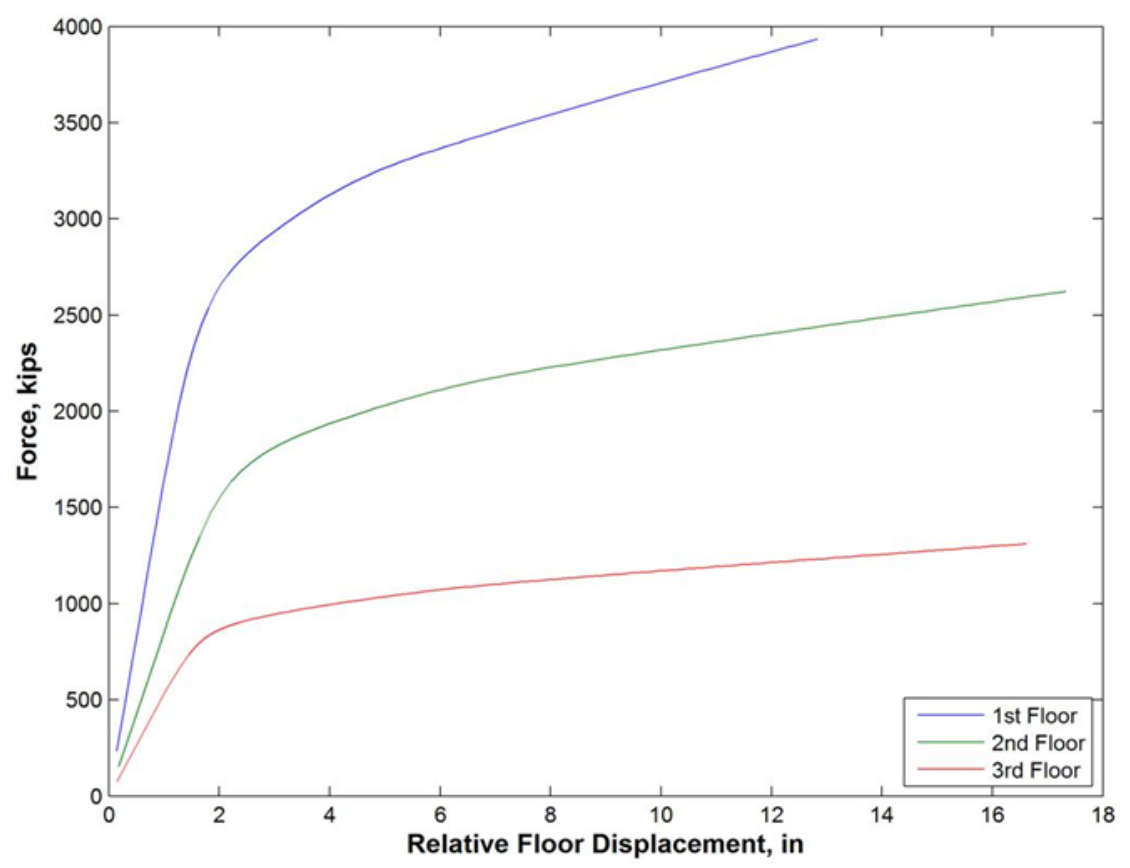

Figure 4.1: Three-Story pushover curves

Similar pushover analysis results were seen for the Los Angeles nine-story and twenty-story models. The pushover curves for the nine and twenty-story models are shown respectively in Figs. 4.2 and 4.3 below. As with the three-story model, all of the stories experience linear behavior below displacements/drifts of two inches as expected. At greater displacements/drifts the columns transition to non-linear behavior and show good strain hardening. These results provide added confidence to the accuracy of the models. 


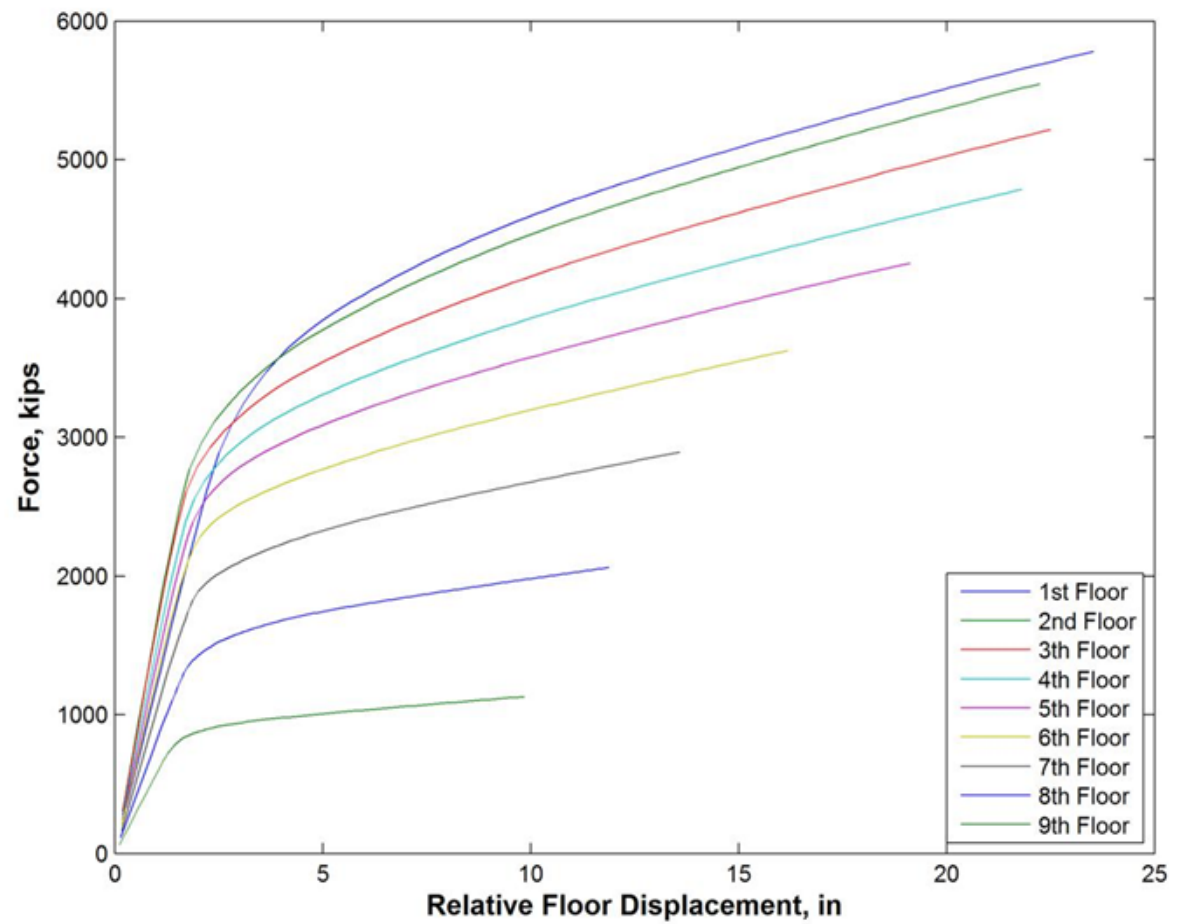

Figure 4.2: Nine-Story pushover curves

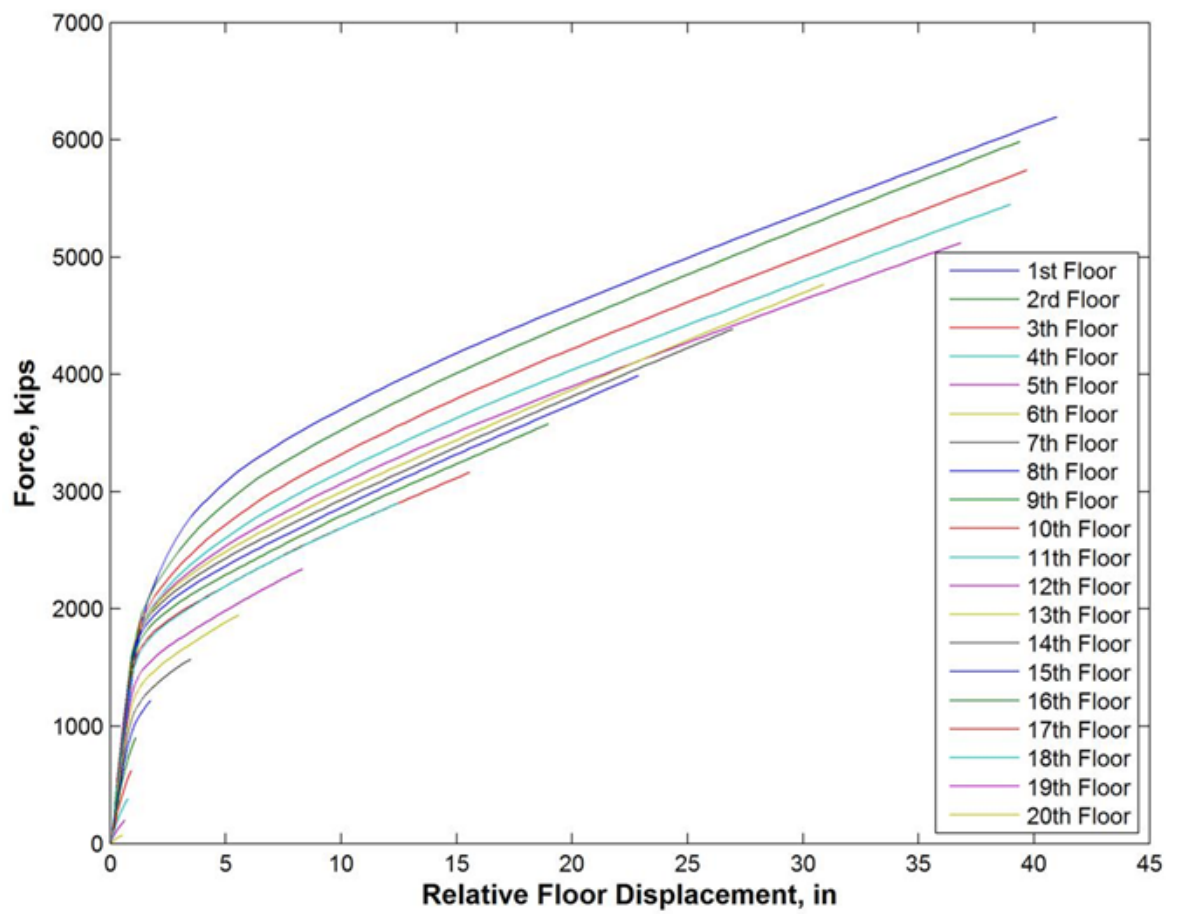

Figure 4.3: Twenty-Story pushover curves 


\section{Chapter 5: Validation of Probabilistic Sampling Method}

With the models completed and validated, a reliable sampling method is needed to accurately capture the drift distributions along the height of the buildings. To achieve accurate results and accurate distributions of story drifts with randomly applied load eccentricities and load intensities, a large number of simulations would be required. With each simulation taking several hours to complete, and given the available computational power, this approach is not feasible here. Alternatively, a point estimate sampling method reduces the required number of simulations drastically while still maintaining accurate results and distributions. For this research the Rosenblueth " $2 \mathrm{k}+1$ " Point Estimate Method will be used. To test the adequacy of this method, the results will be compared against those from a traditional Monte Carlo Simulation. The simple single-story model and the simple three-story model will be evaluated with both techniques, and then the maximum drifts and drift standard deviations will be compared. The small size and minimal complexity of the simple models allows for the required number of simulations to be completed in a reasonable amount of time while still developing accurate drift distributions.

\subsection{Monte Carlo Simulation}

For the traditional Monte Carlo Simulation the three variables with the distributions determined in Chapter 3 were applied randomly to each floor of the models. Each simulation has a random eccentricity along both the $\mathrm{x}$ - and z-axes as well as a random load intensity applied to each floor level. For each simulation these variables are randomly applied and the number of simulations is increased to a sufficient amount until the mean and standard deviation of the story drifts have converged. 


\subsection{Rosenblueth " $2 \mathrm{k}+1$ " Point Estimate Method}

The Rosenblueth " $2 \mathrm{k}+1$ ” Point Estimate Method reduces the number of required simulations down to " $2 \mathrm{k}+1$ " where " $\mathrm{k}$ " is the number of random variables in the entire model. The first simulation is run with each variable at its mean value. Each consecutive simulation changes one variable at a time to plus and minus one standard deviation of the mean value while all other variables are maintained at their respective mean values (Nowak et al., 2000). For this project there are three variables per floor that are being considered - eccentricity along the $\mathrm{x}$ - and z-axes and load intensity - for a total of three variables for the single-story model and nine variables for the three-story model. This corresponds to a total of seven and nineteen simulations for each model, respectively. The maximum drift results from each simulation are then combined to produce a mean value and coefficient of variance. The equations below have been taken from Nowak et al. (2000), and describe the procedure to combine the values from each simulation.

Rosenblueth “2k+1” Point Estimate Method Equations (Nowak et al. 2000):

$$
\begin{aligned}
& Y=f\left(X_{1}, X_{2}, \ldots, X_{K}\right) \\
& y_{o}=f\left(\mu_{X 1}, \mu_{X 2}, \ldots, \mu_{X K}\right) \\
& y_{i}^{+}=f\left(\mu_{X 1}, \mu_{X 2}, \ldots, \mu_{X i}+\sigma_{X i}, \mu_{X K}\right) \\
& y_{i}^{-}=f\left(\mu_{X 1}, \mu_{X 2}, \ldots, \mu_{X i}-\sigma_{X i}, \mu_{X K}\right) \\
& \bar{y}_{i}=\frac{y_{i}^{+}+y_{i}^{-}}{2} \\
& V_{y_{i}}=\frac{y_{i}^{+}-y_{i}^{-}}{y_{i}^{+}+y_{i}^{-}}
\end{aligned}
$$


$\bar{Y}=y_{o} \prod_{i=1}^{K}\left(\frac{\bar{y}_{i}}{y_{o}}\right)$

$V_{Y}=\sqrt{\left(\prod_{i=1}^{K}\left(1+V_{y_{i}}^{2}\right)\right)-1}$

The Rosenblueth " $2 \mathrm{k}+1$ ” Point Estimate Method generates a mean value, $\mu$, and a coefficient of variation, $\mathrm{c}_{\mathrm{v}}$, for each set of simulations, equations 5.7 and 5.8 respectively. These values are multiplied together to determine the standard deviation, $\sigma$, which is used to represent the distribution for each set of simulations:

$$
\text { Standard Deviation: } \quad \sigma=c_{v} \mu
$$

\subsection{Comparison}

The results of each of these methods are directly compared to one another. The three variables of concern, eccentricity along the $\mathrm{x}$ - and z-axes and load intensity, will be applied to the two building models. In addition, different ground motions at different intensities will also be applied to test the validity of the sampling method over the non-linear range. Consistently similar results between the two methods will validate the adequacy of the point estimate sampling method.

\subsubsection{Simple Single-Story Model}

The simple single-story model was tested with three different ground motions at three different ground motion intensities. The ground motions were applied along both plan dimensions of the building for a total of six different loading scenarios. For each scenario, 500 Monte Carlo Simulations were run, the drifts from Monte Carlo Simulations were averaged and the mean 
values and standard deviations were calculated as normal. The drifts for the Rosenblueth " $2 \mathrm{k}+1$ " Point Estimate Method simulation were combined in accordance with the previously mentioned equations in Section 5.2. The mean values and coefficients of variation generated from the point estimate simulations were then combined to determine the standard deviations.

The mean maximum story drifts from the Monte Carlo Simulation are plotted against those from the Rosenblueth Simulation in Fig. 5.1 below. The mean drift values of the Monte Carlo Simulations are plotted along the $\mathrm{x}$-axis and the predicted mean drift values from the Rosenblueth " $2 \mathrm{k}+1$ " Simulations are plotted along the $\mathrm{y}$-axis. The points fall along a straight line with a slope of one; this shows a good one to one relationship between the two sampling methods.

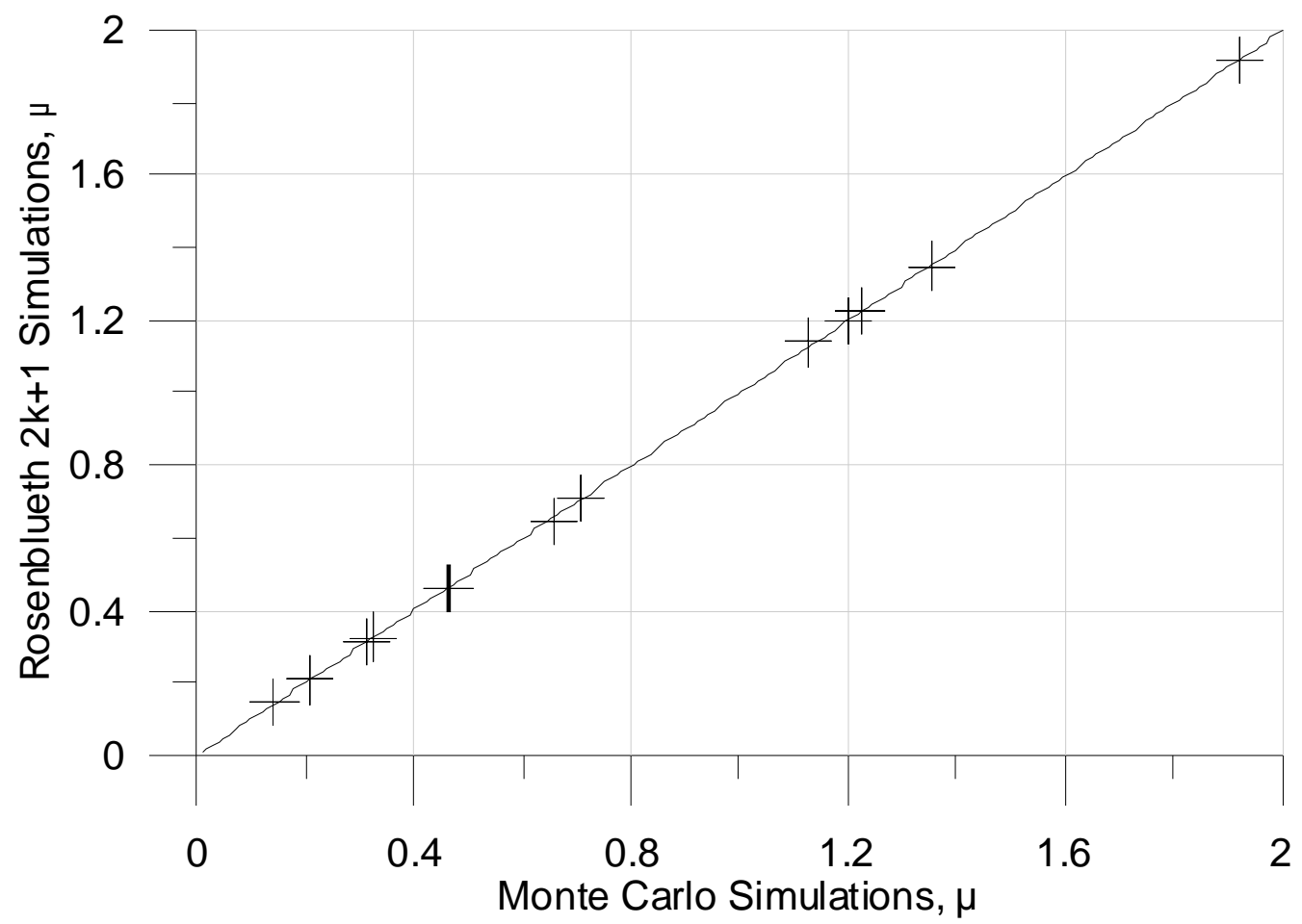

Figure 5.1: Single-story mean drift comparison

Similarly, the standard deviations for the two methods were plotted against each other in Fig. 5.2. The plot of the standard deviations also shows a nearly one to one comparison, which adds further confidence to the accuracy of the Rosenblueth " $2 \mathrm{k}+1$ " Point Estimate Method. The 500 
hundred simulations required for each scenario to achieve adequate results for the single story model has been reduced down to just seven simulations that accurately capture and represent the true distribution determined from the Monte Carlo Simulation.

The data from the Figs. 5.1 and 5.2 is tabulated in Table 5.1. The Monte Carlo Simulation values can be directly compared to the Rosenblueth " $2 \mathrm{k}+1$ " Simulation values.

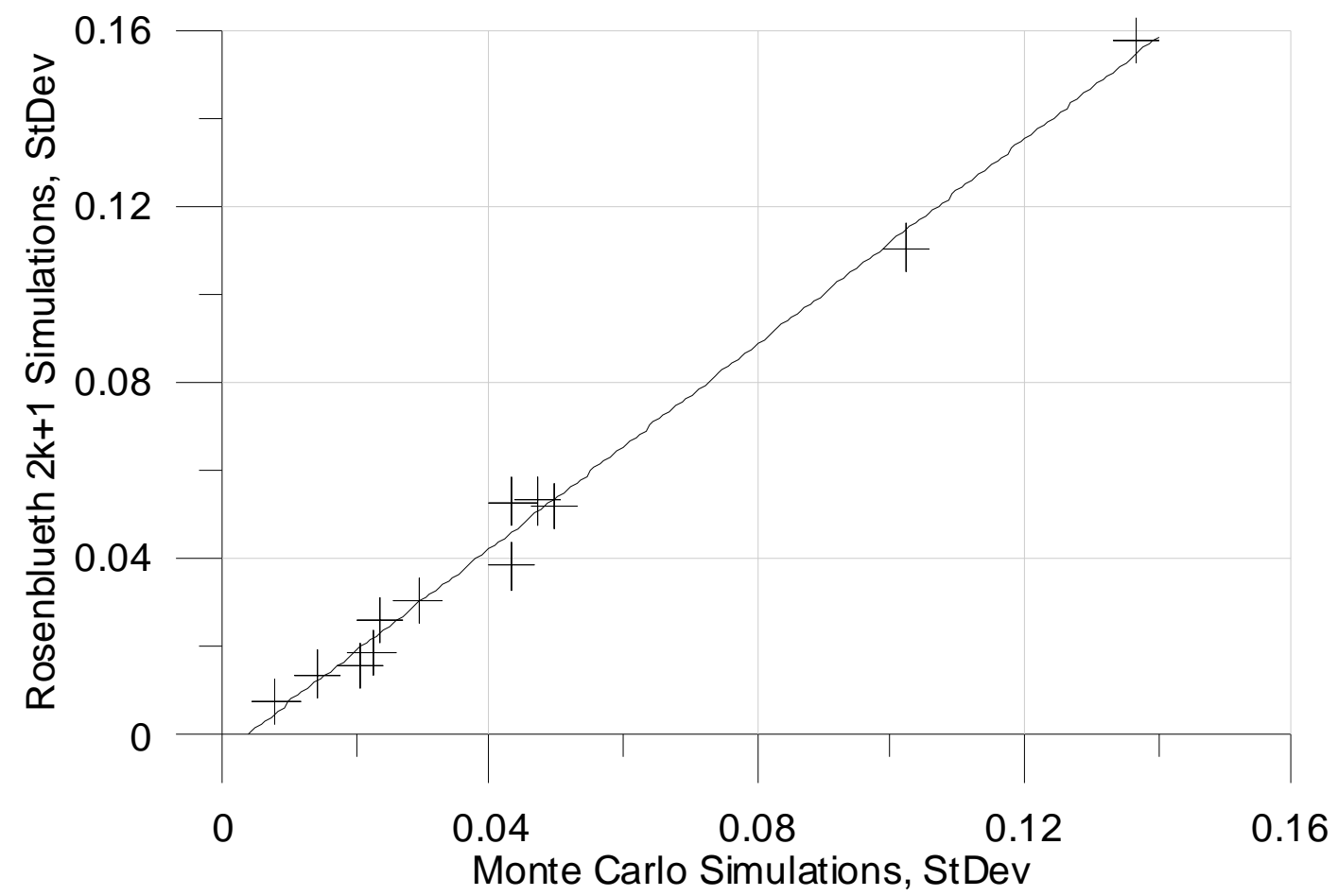

Figure 5.2: Single-story standard deviation drift comparison 
Table 5.1: Simple single-story model sampling method comparison

\begin{tabular}{|c|c|c|c|c|c|c|}
\hline \multirow[b]{2}{*}{ Ground Motions } & \multirow{2}{*}{$\begin{array}{l}\text { Ground } \\
\text { Motion } \\
\text { Directions }\end{array}$} & \multirow[b]{2}{*}{ Drift } & \multicolumn{2}{|c|}{ Monte Carlo Simulations } & \multicolumn{2}{|c|}{ Rosenblueth, $2 \mathrm{k}+1$} \\
\hline & & & $\begin{array}{l}\text { Mean } \\
(\mu)\end{array}$ & $\begin{array}{l}\text { Standard } \\
\text { Deviation }(\sigma)\end{array}$ & $\begin{array}{l}\text { Mean } \\
(\mu)\end{array}$ & $\begin{array}{l}\text { Standard } \\
\text { Deviation }(\sigma)\end{array}$ \\
\hline \multirow{4}{*}{$\begin{array}{l}\text { 3_Duzce Turkey, } \\
\mathrm{PS} \mathrm{a}=0.3 \mathrm{~g}\end{array}$} & Primary & $\mathrm{x}$ & 0.1434 & 0.0141 & 0.1465 & 0.0139 \\
\hline & & $\mathrm{z}$ & 0.7096 & 0.0293 & 0.7098 & 0.0308 \\
\hline & Minor & $\mathrm{x}$ & 0.2084 & 0.0079 & 0.2079 & 0.0078 \\
\hline & & $\mathrm{z}$ & 0.3119 & 0.0235 & 0.3128 & 0.0263 \\
\hline \multirow{4}{*}{$\begin{array}{l}\text { 1_Northridge, } \\
\mathrm{PS} a=1.0 \mathrm{~g}\end{array}$} & Primary & $x$ & 0.3270 & 0.0224 & 0.3249 & 0.0188 \\
\hline & & $\mathrm{z}$ & 1.1271 & 0.0431 & 1.1388 & 0.0385 \\
\hline & Minor & $\mathrm{x}$ & 0.4643 & 0.0434 & 0.4627 & 0.0530 \\
\hline & & $\mathrm{z}$ & 1.2004 & 0.0495 & 1.1979 & 0.0521 \\
\hline \multirow{4}{*}{$\begin{array}{l}\text { 2_Northridge, } \\
\mathrm{PS} a=2.0 \mathrm{~g}\end{array}$} & Primary & $x$ & 1.2235 & 0.0206 & 1.2255 & 0.0158 \\
\hline & & $\mathrm{z}$ & 1.3554 & 0.1024 & 1.3482 & 0.1106 \\
\hline & Minor & $\mathrm{x}$ & 0.6597 & 0.0472 & 0.6428 & 0.0533 \\
\hline & & $\mathrm{z}$ & 1.9231 & 0.1367 & 1.9154 & 0.1576 \\
\hline
\end{tabular}

\subsubsection{Simple Three-Story Model}

The simple three-story model was tested with a single ground motion with the primary component applied along both of the plan dimensions of the model. Due to the increased computational time of the three-story model the ground motion was only applied at one intensity level. A total of 1000 Monte Carlo Simulations were run for each of the two loading scenarios. As with the single-story model, the mean and standard deviations of the two methods for the three-story model were also plotted against each other. Fig. 5.3 below shows the mean drift comparison between the two sampling methods. The mean drift comparison, like the single-story mean comparison, is one to one. Again, the standard deviation comparison, displayed in Fig. 5.4 below, is linear but slightly steeper than one to one. The Rosenblueth Method suggests slightly larger standard deviations which result in a slightly flatter and more spread out distribution. However, the difference is minor and the main result of interest for this research is the mean drift 
value. Therefore, the results are accurately captured with the Rosenblueth " $2 \mathrm{k}+1$ " Point Estimate Method Simulations.

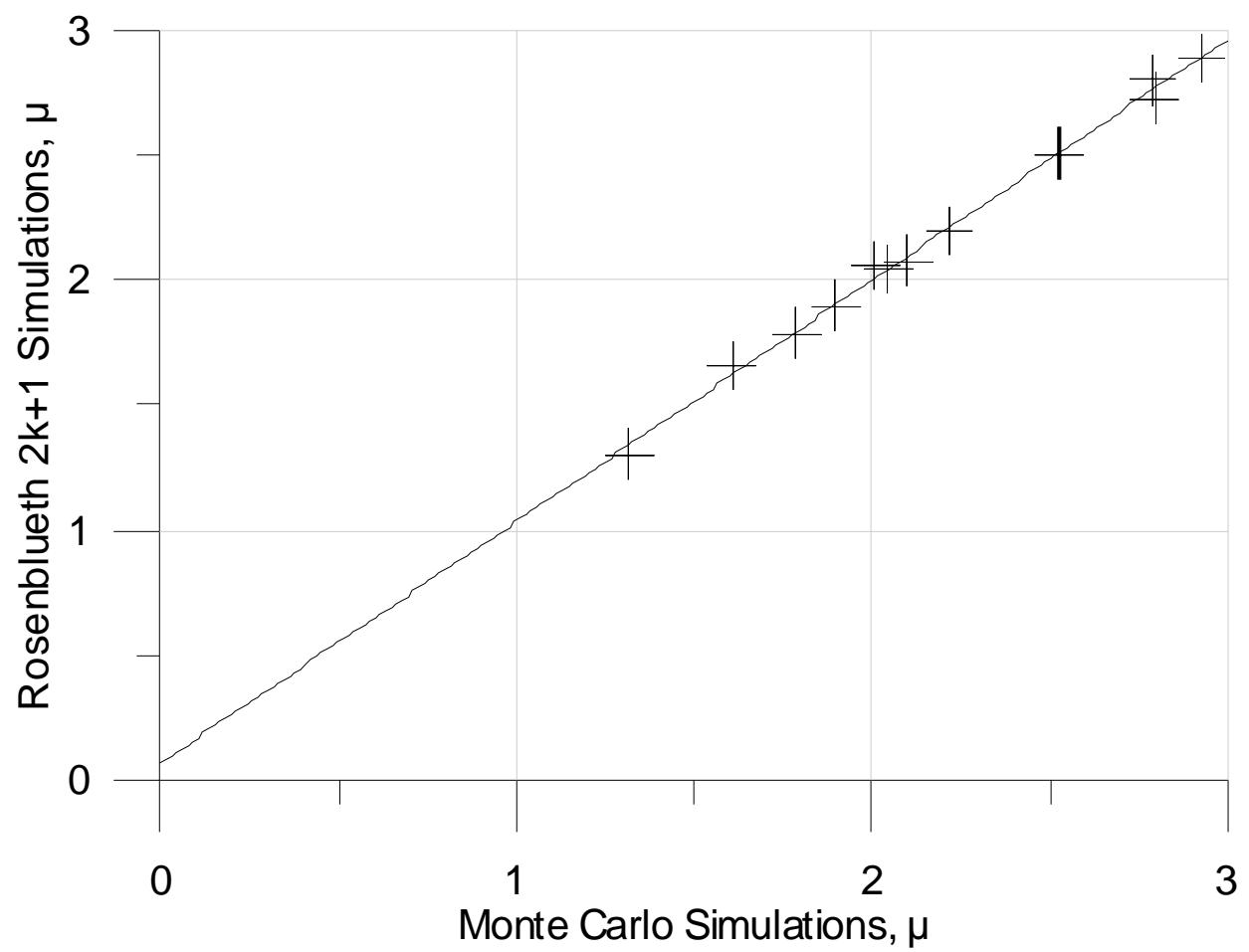

Figure 5.3: Three-story mean drift comparison

The data from Figs. 5.3 and 5.4 is tabulated in Table 5.2. The Monte Carlo Simulation values can be directly compared to the Rosenblueth Simulation values. Comparing the standard deviation values it is clear that on average the Rosenblueth Simulations predict slightly greater values. 


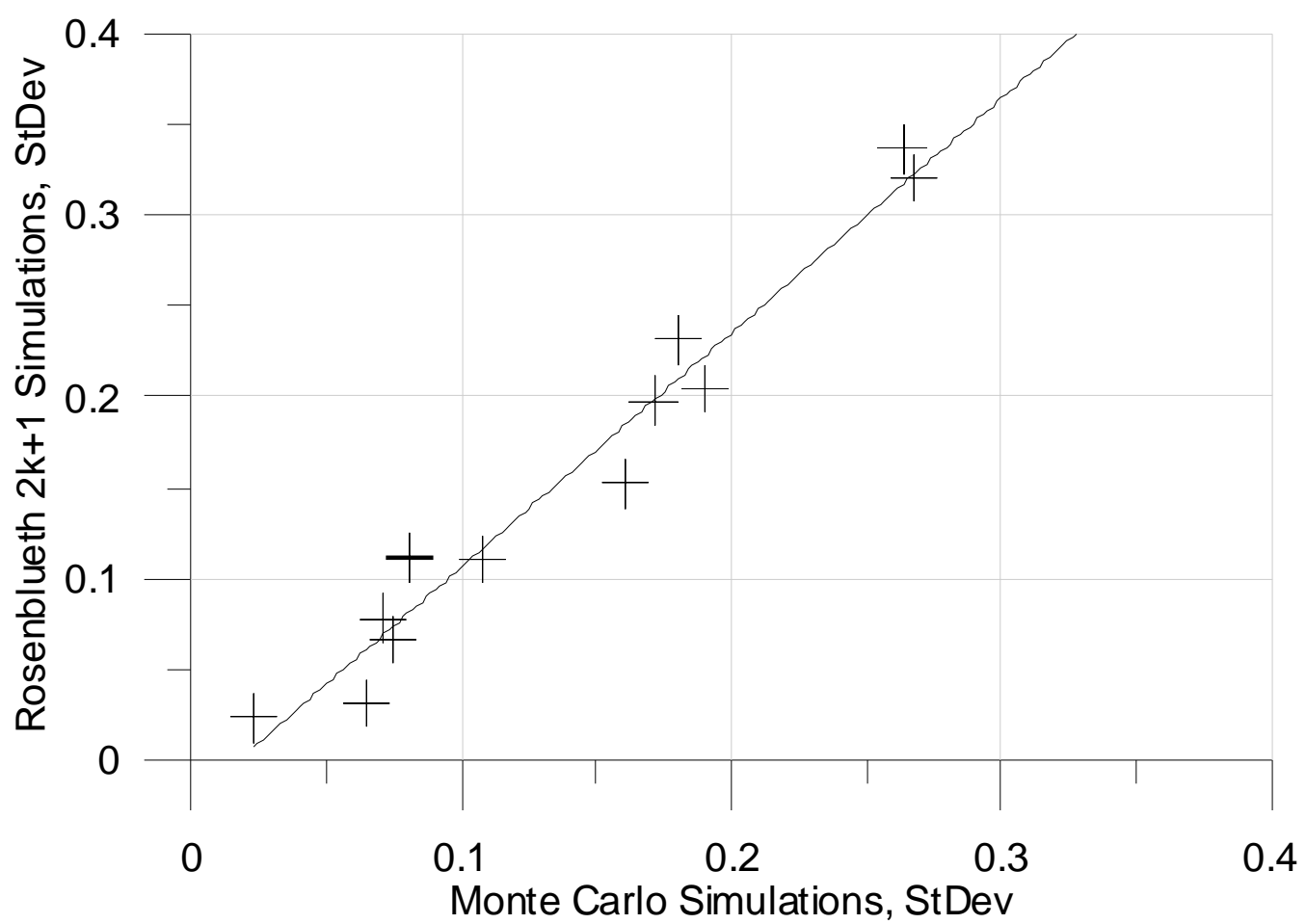

Figure 5.4: Three-story standard deviation drift comparison

Table 5.2: Simple three-story model sampling method comparison

\begin{tabular}{|c|c|c|c|c|c|c|c|}
\hline \multirow[b]{2}{*}{$\begin{array}{l}\text { Ground } \\
\text { Motions }\end{array}$} & \multirow[b]{2}{*}{$\begin{array}{l}\text { Ground } \\
\text { Motion } \\
\text { Direction }\end{array}$} & \multirow[b]{2}{*}{$\begin{array}{l}\text { Floor } \\
\text { Level }\end{array}$} & \multirow[b]{2}{*}{$\begin{array}{l}\text { Drift } \\
\text { (in) }\end{array}$} & \multicolumn{2}{|c|}{$\begin{array}{l}\text { Monte Carlo } \\
\text { Simulations }\end{array}$} & \multicolumn{2}{|c|}{ Rosenblueth, $2 \mathrm{k}+1$} \\
\hline & & & & $\begin{array}{l}\text { Mean } \\
(\mu)\end{array}$ & $\begin{array}{l}\text { Standard } \\
\text { Deviation }(\sigma)\end{array}$ & $\begin{array}{l}\text { Mean } \\
(\mu)\end{array}$ & $\begin{array}{l}\text { Standard } \\
\text { Deviation } \\
(\sigma)\end{array}$ \\
\hline \multirow{12}{*}{$\begin{array}{l}\text { 1_Northridge } \\
\text { Psa }=1.0 \mathrm{~g}\end{array}$} & Primary & 1 & $\mathrm{x}$ & 1.3179 & 0.0709 & 1.3036 & 0.0782 \\
\hline & & 2 & $\mathrm{x}$ & 2.2163 & 0.1076 & 2.1978 & 0.1109 \\
\hline & & 3 & $\mathrm{x}$ & 1.7889 & 0.0744 & 1.7877 & 0.0664 \\
\hline & & 1 & $\mathrm{z}$ & 1.8988 & 0.0226 & 1.8964 & 0.0231 \\
\hline & & 2 & $\mathrm{z}$ & 2.7927 & 0.0649 & 2.7988 & 0.0312 \\
\hline & & 3 & $\mathrm{z}$ & 2.0476 & 0.0807 & 2.0449 & 0.1115 \\
\hline & Minor & 1 & $\mathrm{x}$ & 1.6063 & 0.1605 & 1.6611 & 0.1522 \\
\hline & & 2 & $\mathrm{x}$ & 2.7959 & 0.2677 & 2.7267 & 0.3200 \\
\hline & & 3 & $\mathrm{x}$ & 2.5274 & 0.1900 & 2.5038 & 0.2044 \\
\hline & & 1 & $\mathrm{z}$ & 2.1025 & 0.1801 & 2.0774 & 0.2315 \\
\hline & & 2 & $\mathrm{z}$ & 2.9283 & 0.2633 & 2.8899 & 0.3363 \\
\hline & & 3 & $\mathrm{z}$ & 2.0106 & 0.1712 & 2.0556 & 0.1977 \\
\hline
\end{tabular}


Because the Rosenblueth " $2 \mathrm{k}+1$ " Point Estimate Method has demonstrated an ability to reduce the hundreds to thousands of simulations down to just a few simulations while still accurately representing the mean and standard deviation for each scenario, this method will be used to evaluate each of the three Los Angeles models. This reduces the number of required simulations down to nineteen for the three-story building, fifty-five for the nine-story model, and one hundred twenty-one for the twenty-story model. These values are listed in Table 5.3 below and correspond to one set of ground motions applied along a single plan dimension.

Table 5.3: Required Rosenblueth " $2 \mathrm{k}+1$ " simulations for each ground motion

\begin{tabular}{llll}
\hline & Variables/Floor & Total Variables & Total Simulations \\
\hline 3-Story & 3 & 9 & 19 \\
9-Story & 3 & 27 & 55 \\
20-Story & 3 & 60 & 121 \\
\hline
\end{tabular}




\section{Chapter 6: Parametric Study}

With all of the preliminary work and necessary validations now complete, the next step is to establish all the variables for the parametric analyses. Of which, the main variables that are essential to the incremental dynamic analysis are the ground motions. All of the ground motions are similarly normalized so they can be applied in an incremental dynamic analysis.

\subsection{Ground Motions: Normalization}

The ground motions applied to the models were taken from FEMA document P-695

Quantification of Building Seismic Performance Factors. Table 6.1 below was taken directly from FEMA P-695 and lists the twenty-two far-field ground motions that are applied to the models (FEMA P-695, 2009, p. A-14).

The pair of horizontal ground motion acceleration time histories for each of the twenty-two ground motions was downloaded through the online PEER Strong Motion Database: peer.berkeley.edu/smcat/search/html. 
Table 6.1: Far-field ground motions

\begin{tabular}{|c|c|c|c|c|c|}
\hline \multirow{2}{*}{$\begin{array}{l}\text { ID } \\
\text { No. }\end{array}$} & \multicolumn{3}{|c|}{ Earthquake } & \multicolumn{2}{|c|}{ Recording Station } \\
\hline & M & Year & Name & Name & Owner \\
\hline 1 & 6.7 & 1994 & Northridge & Beverly Hills - Mulhol & USC \\
\hline 2 & 6.7 & 1994 & Northridge & Canyon Country-WLC & USC \\
\hline 3 & 7.1 & 1999 & Duzce, Turkey & Bolu & ERD \\
\hline 4 & 7.1 & 1999 & Hector Mine & Hector & SCSN \\
\hline 5 & 6.5 & 1979 & Imperial Valley & Delta & UNAMUCSD \\
\hline 6 & 6.5 & 1979 & Imperial Valley & El Centro Array \#11 & USGS \\
\hline 7 & 6.9 & 1995 & Kobe, Japan & Nishi-Akashi & CUE \\
\hline 8 & 6.9 & 1995 & Kobe, Japan & Shin-Osaka & CUE \\
\hline 9 & 7.5 & 1999 & Kocaeli, Turkey & Duzce & ERD \\
\hline 10 & 7.5 & 1999 & Kocaeli, Turkey & Arcelik & KOERI \\
\hline 11 & 7.3 & 1992 & Landers & Yermo Fire Station & CDMG \\
\hline 12 & 7.3 & 1992 & Landers & Coolwater & SCE \\
\hline 13 & 6.9 & 1989 & Loma Prieta & Capitola & CDMG \\
\hline 14 & 6.9 & 1989 & Loma Prieta & Gilroy Array \#3 & CDMG \\
\hline 15 & 7.4 & 1990 & Manjil, Iran & Abbar & $\mathrm{BHRC}$ \\
\hline 16 & 6.5 & 1987 & Superstition Hills & EI Centro Imp. Co. & CDMG \\
\hline 17 & 6.5 & 1987 & Superstition Hills & Poe Road (temp) & USGS \\
\hline 18 & 7.0 & 1992 & Cape Mendocino & Rio Dell Overpass & CDMG \\
\hline 19 & 7.6 & 1999 & Chi-Chi, Taiwan & CHY101 & CWB \\
\hline 20 & 7.6 & 1999 & Chi-Chi, Taiwan & TCU045 & CWB \\
\hline 21 & 6.6 & 1971 & San Fernando & LA - Hollywood Stor & CDMG \\
\hline 22 & 6.5 & 1976 & Friuli, Italy & Tolmezzo & -- \\
\hline
\end{tabular}

The Elastic Response Spectra for each of the forty-four ground motion components were created with SeismoSignal, a product of SeismoSoft, and the response spectrums for each component were calculated with a $5.0 \%$ damping value. The ground motions were adjusted in accordance with FEMA 350 A.3.1, Detailed Procedures for Performance Evaluation (FEMA 350, 2000). The intensity of each ground motion pair was normalized by one of the components at the fundamental period of the building being analyzed. Both ground motion pairs were equally adjusted by the same factor so that when normalized the pseudo-spectral acceleration of one ground motion component was equal to $1.0 \mathrm{~g}$ at the fundamental period of the building of interest. The other ground motion component would have a pseudo-spectral acceleration less than or equal to $1.0 \mathrm{~g}$ at that same fundamental period. The component that is equal to $1.0 \mathrm{~g}$ at the fundamental 
period is referred to as the primary ground motion component, and the other component is the minor ground motion. Each of the ground motions were then applied in incremental intensities for an incremental dynamic analysis. Below Fig. 6.1 shows the primary and minor components of the first ground motion, Northridge (Station: Mulhol), normalized for the 3-Story model.

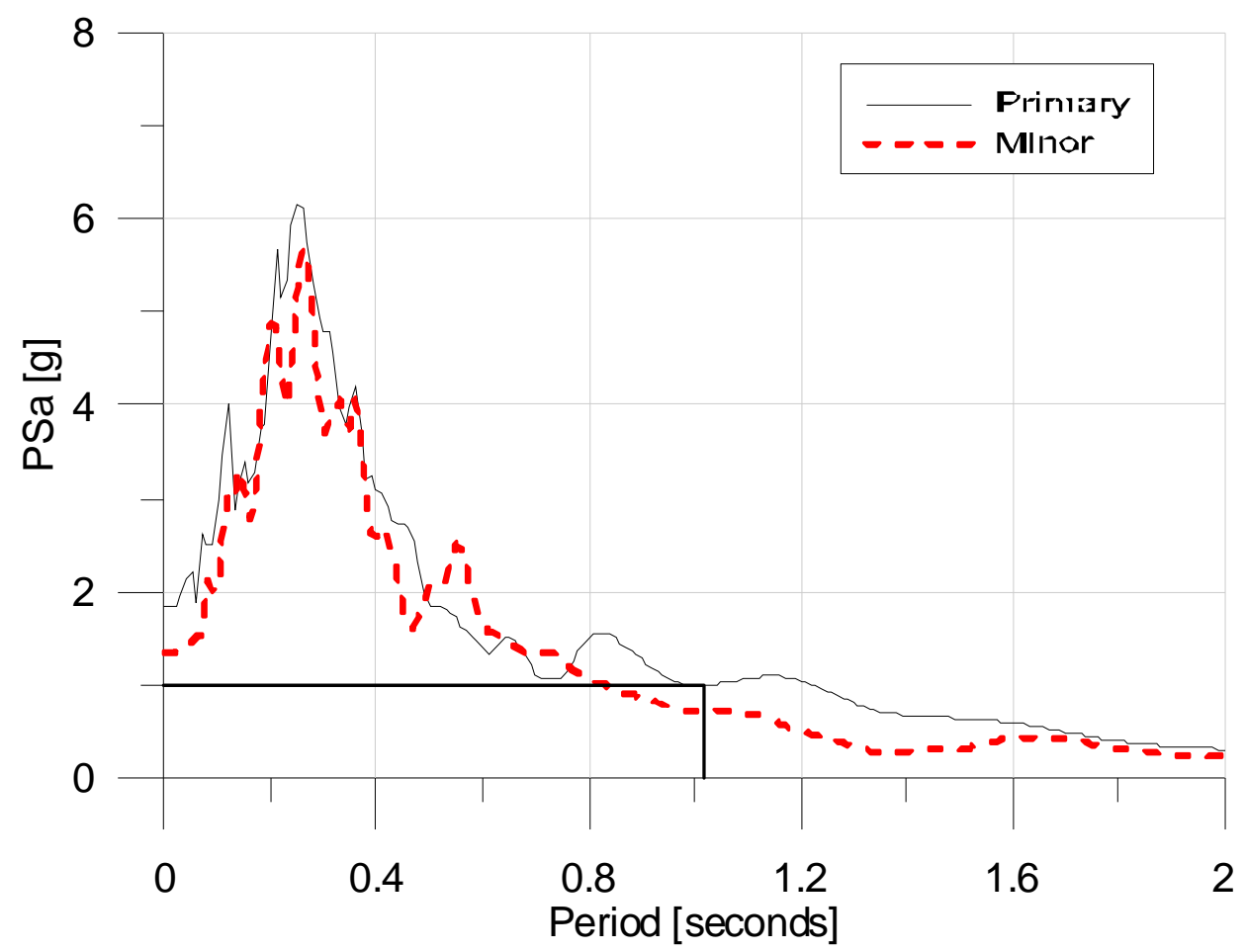

Figure 6.1: Northridge (Station: Mulhol) spectral acceleration components

The primary component (represented by the solid line) has been normalized to the value of $1.0 \mathrm{~g}$ at a period of 1.02 seconds, which is the fundamental period of the three-story Los Angeles model. The minor component (represented by the dashed line) has a value less than $1.0 \mathrm{~g}$ at that same period.

Earthquakes are a source of great variability, and in an attempt to minimize the uncertainty associated with the ground motion variable, all twenty-two motions have been chosen to analyze each model. This will increase the computational time but will more accurately capture the effects of the ground motions variability (Chang et al., 2009). 


\subsection{Floor Load Intensities and Load Eccentricities}

Floor load intensities and load eccentricities values are described in Tables A.1-A.4 of Appendix A.

\subsection{Computer Specifications, Computational Time, and Residual Tolerance}

For this parametric study, all of the simulations were performed in the Civil Engineering Student Computer Lab \#2, CE 13-117, on the Cal Poly San Luis Obispo campus. The specifications for the computers in the lab are listed in Table 6.2 below.

Table 6.2: Computer information

\begin{tabular}{lll}
\hline CE 13-117 Computer Lab & \\
\hline Computer & Processor & Intel (R) Core (TM) i5-2400 CPU @ 3.10 GHz \\
& Ram & 4.00 GB (3.24 GB usable) \\
& System & Window 7-32 bit \\
\hline
\end{tabular}

These machines run on quad processors and are capable of efficiently running four simultaneous simulations. Table 6.3 below summarizes the time required to run each model. The durations listed in the final column are estimates per ground motion intensity. Greater intensities will cause more nonlinear deformation of the models and take longer to solve while lower intensities will consequently have less nonlinear deformation and will be solved more quickly.

Table 6.3: Required simulation computational time

\begin{tabular}{|c|c|c|c|c|c|c|}
\hline Model & $\begin{array}{l}\text { Ground } \\
\text { Motions }\end{array}$ & $\begin{array}{l}\text { Applied } \\
\text { Directions }\end{array}$ & $\begin{array}{l}\text { Rosenblueth } \\
\text { Simulations }\end{array}$ & $\begin{array}{l}\text { Total } \\
\text { Simulations }\end{array}$ & $\begin{array}{l}\text { Hours } \\
\text { /Simulation* }\end{array}$ & $\begin{array}{l}\text { Total Days } \\
\text { /Computer** }\end{array}$ \\
\hline 3_Story & 22 & 2 & 19 & 836 & 0.046 & 0.4 \\
\hline 9_Story & 22 & 1 & 55 & 1210 & 1.543 & 19.5 \\
\hline 20 Story & 22 & 1 & 121 & 2662 & 3.500 & 97.1 \\
\hline
\end{tabular}

*Approximate duration based on average ground motion duration and intensity level.

**This assumes each computer is running four simultaneous simulations. 
Each consecutive model not only requires more Rosenblueth Simulations, but the increased complexity of each model also increases the computational time per simulation. Table 6.4 summarizes the number of nodes and elements in each of the three models. The three-story model has been chosen to be analyzed with the bi-directional ground motions applied in two directions: the first with the primary ground motion component parallel with the x-direction, and the second with the primary component parallel with the z-direction. The nine-story model is perfectly symmetric and only required that the bi-directional ground motions be applied in one direction. The twenty-story model is not symmetric, however, due to the long simulation run time, the bidirectional ground motions are only applied along one direction.

Table 6.4: Model complexity information

\begin{tabular}{lll}
\hline Model & Total Nodes & Total Elements \\
\hline 3_Story & 70 & 84 \\
9_Story & 280 & 440 \\
20_Story & 638 & 1100 \\
\hline
\end{tabular}

The increased complexity of the nine-story and twenty-story models greatly increases the computational time. Originally, all of the models had a residual solver tolerance of $1 \mathrm{e}-06$. This value was maintained for the three-story model because it was the least computationally intensive of the three models. The nine-story residual solver tolerance was increased to $1 \mathrm{e}-04$. The relaxation of the tolerance greatly reduced the computational time with negligible effect to the output data. The twenty-story residual solver tolerance needed to be increased to $1 \mathrm{e}-02$ in order to complete the simulations in a reasonable timeframe. The durations listed in Table 6.3 above are the durations corresponding to the models with the respective increased tolerances.

The relaxation of the residual solver tolerance is not expected to affect the results. Several simulations were run and the results were compared to show that the solutions were within reason. In Fig. 6.2 below, the nine-story model floor displacements for several simulations are 
compared at different residual tolerances. The $\mathrm{x}$-axis corresponds to the original displacements solved with the default residual tolerance of $1.0 \mathrm{e}-06$. The y-axis has the same displacements solved with an increased residual tolerance of $1.0 \mathrm{e}-04$. The results are a near perfect one to one relationship.

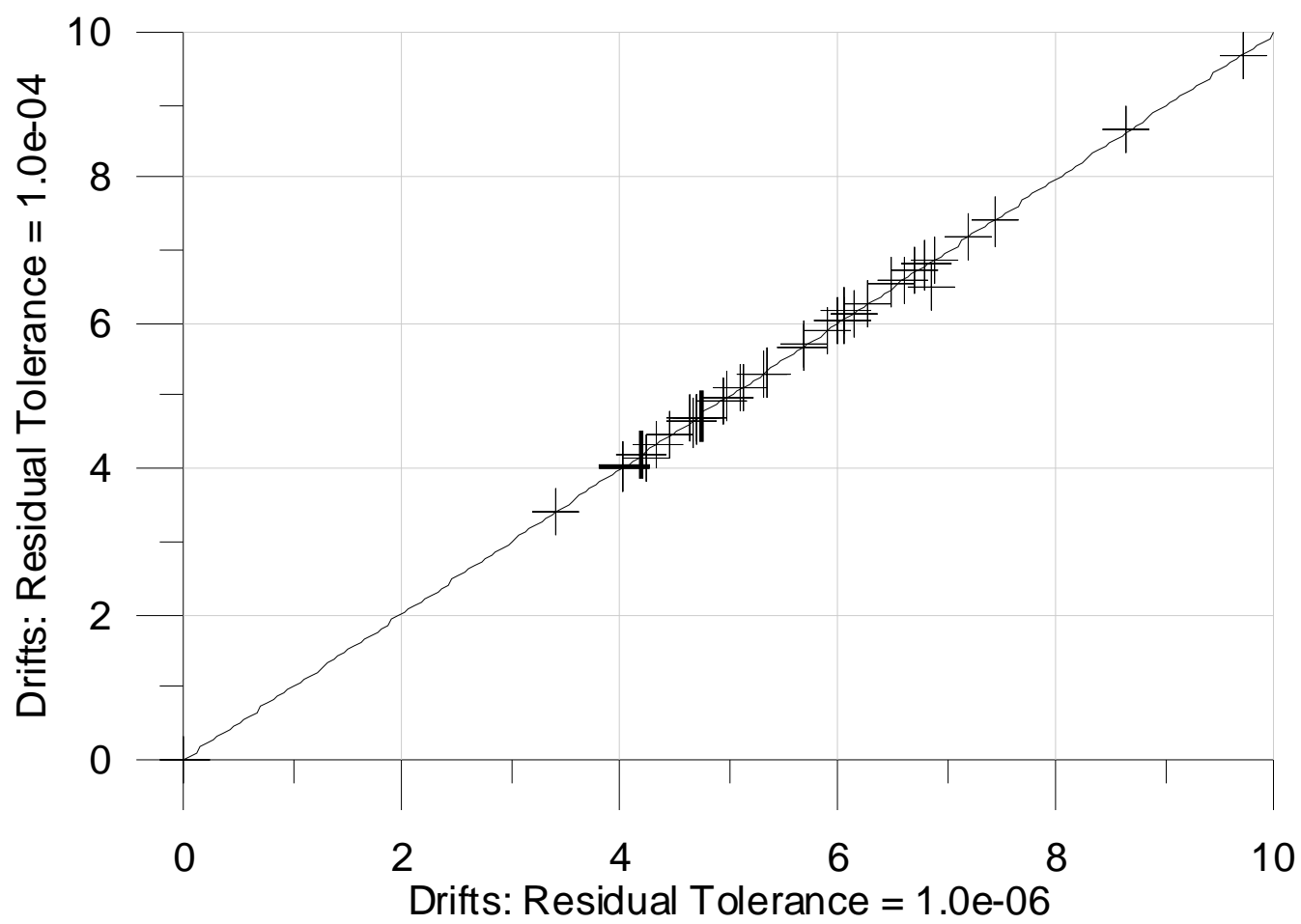

Figure 6.2: Nine-story tolerance reduction validation, 3.3 times faster

In Fig. 6.3 below, the twenty-story floor displacements for several simulations are compared at different residual tolerances. Again the x-axis corresponds to the displacements solved with the original residual tolerance of 1.0e-06. The y-axis has the displacements solved for with a residual tolerance of 1.0e-02. Again the results show a one to one relationship between the two residual tolerances. 


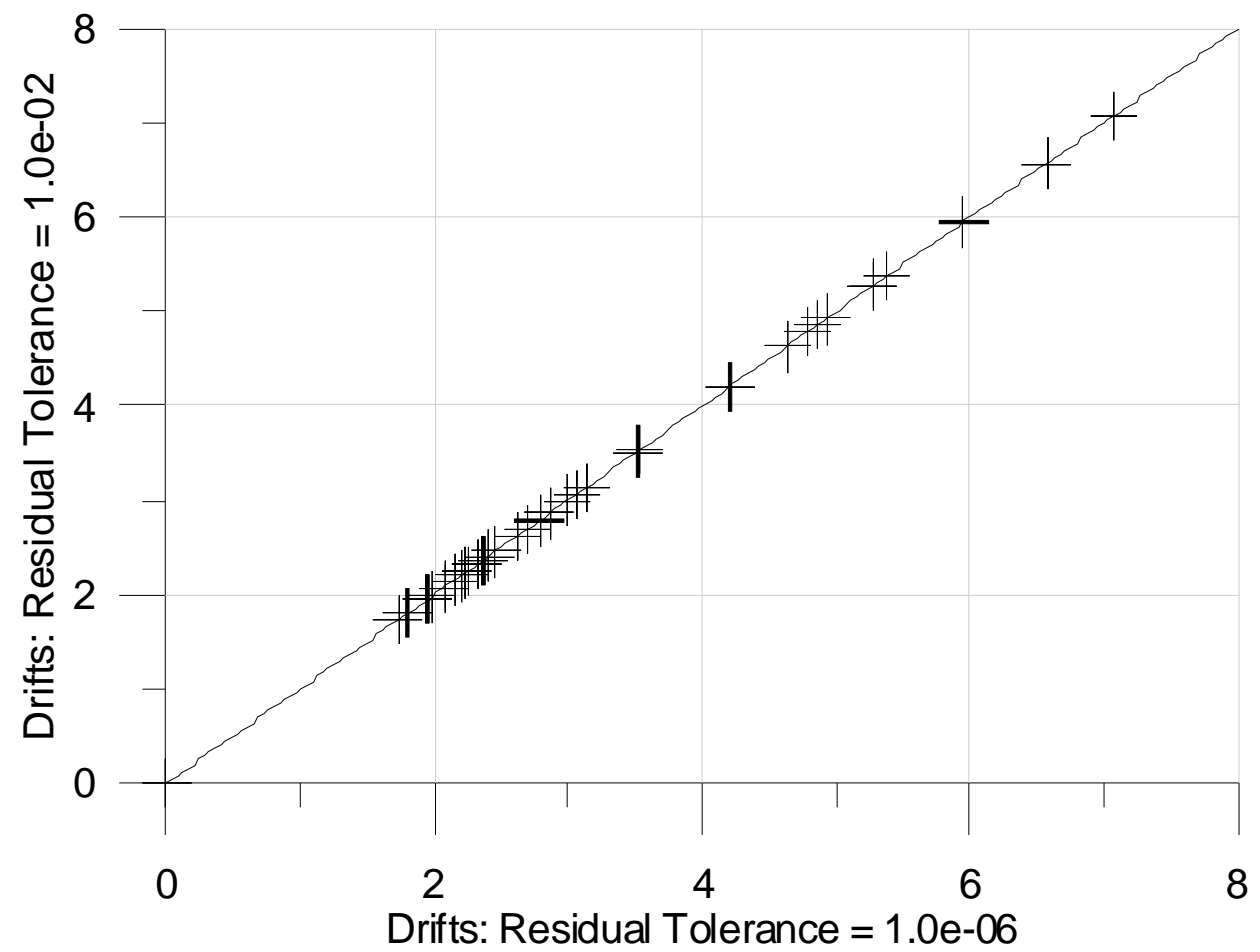

Figure 6.3: Twenty-story tolerance reduction validation, 2.3 times faster

The reduction of the residual tolerances of the nine-story and twenty-story models has negligible to no effect on the recorded floor displacements. However, the reduction does greatly reduce the computational time per simulation, on average 3.3 times faster for the nine-story model, and 2.3 times faster for the twenty-story model. 


\section{Chapter 7: Results and Analysis}

Now that all of the required simulations have been completed, the inter-story drifts can be analyzed. The probabilistic distribution type of the inter-story drifts needs to be determined before the desired fragility analyses can be performed. The Kolmogorov-Smirnov test for goodness-of-fit compares the drifts against a lognormal and normal distribution.

\subsection{Kolmogorov-Smirnov Test for Goodness-of-Fit: LogNormal vs. Normal}

The maximum inter-story drifts for all of the simulations were combined with the Rosenblueth “ $2 \mathrm{k}+1$ ” Point Sampling Method equations, outlined in Section 5.2, to generate the expected maximum drifts. The expected drifts at each level of ground shaking intensity were evaluated with the Kolmogorov-Smirnov (K-S) Test for Goodness-of-Fit to find the proper probabilistic distribution that the maximum inter-story drifts follow. Details on the procedure of the K-S Test can be found in Ang et al. (2007). The K-S test essentially compares the given distribution of drifts against the theoretical distributions predicted by a normal set and lognormal set. The set with the least maximum difference between the actual and theoretical drifts is considered the better fit. Figs. 7.1, 7.2, and 7.3 below are the results of the K-S test for each of the three models over their respective intensity ranges. The $y$-axis, $D_{n}$, is the absolute difference between the theoretical drifts and the actual drifts.

For each of the building models, the lognormal distribution of the maximum drifts consistently provides smaller values of $D_{n}$ than the normal distribution, indicating lognormal distribution better represents the probabilistic distributions of the data. Therefore, when calculating the fragility curves a lognormal distribution of the drift values is more appropriate. 


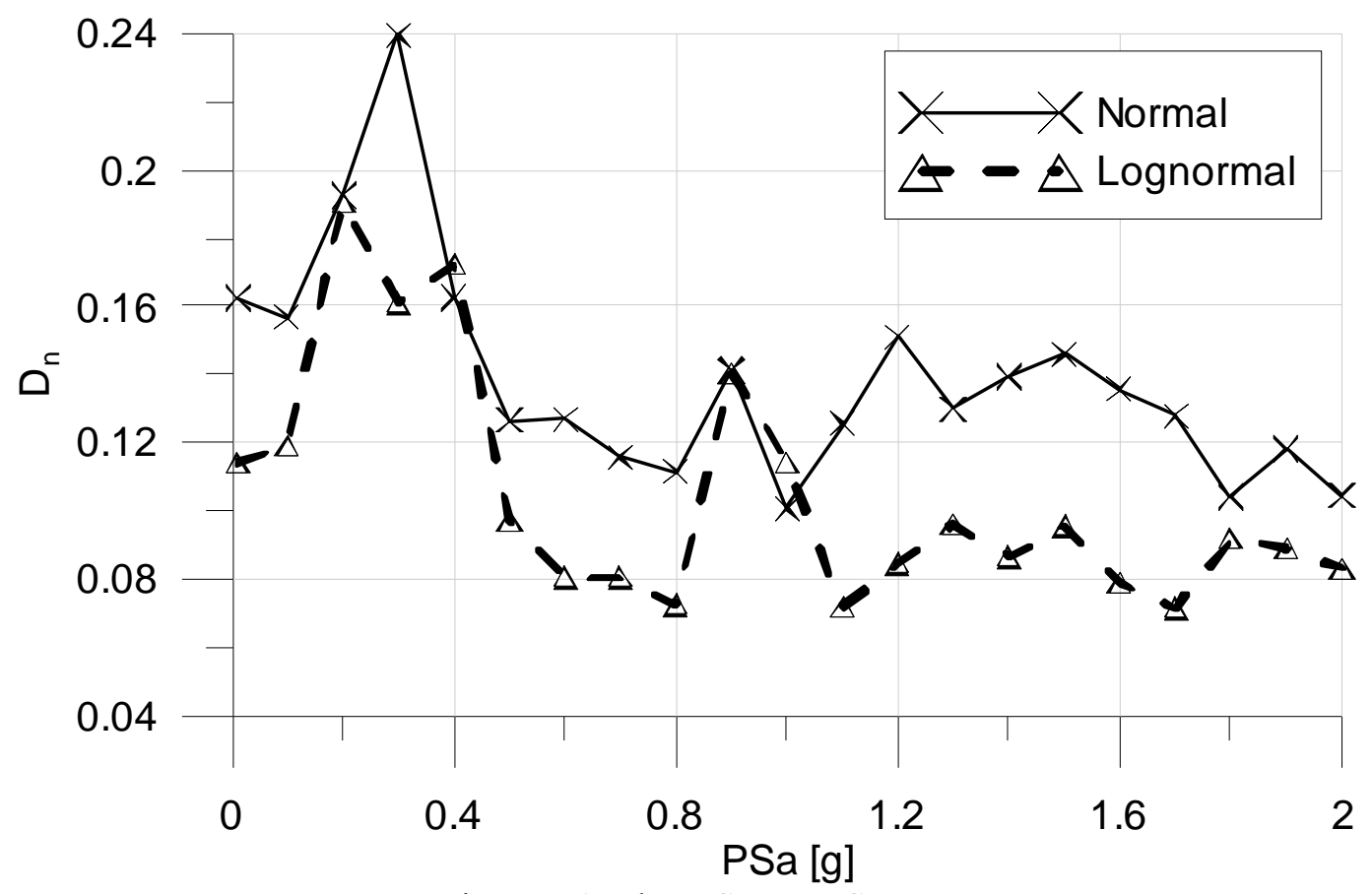

Figure 7.1: Three-Story K-S test

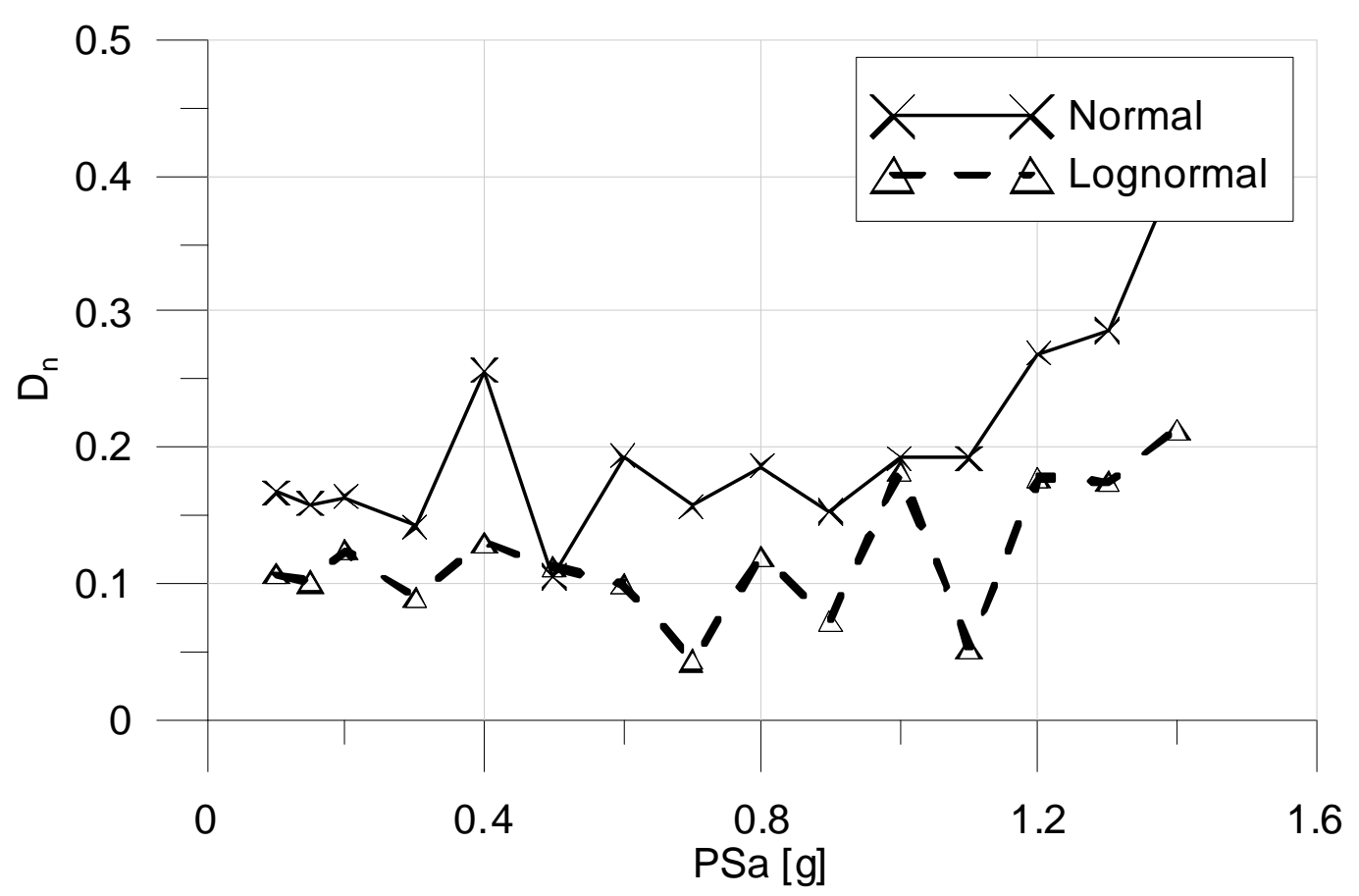

Figure 7.2: Nine-Story K-S test 


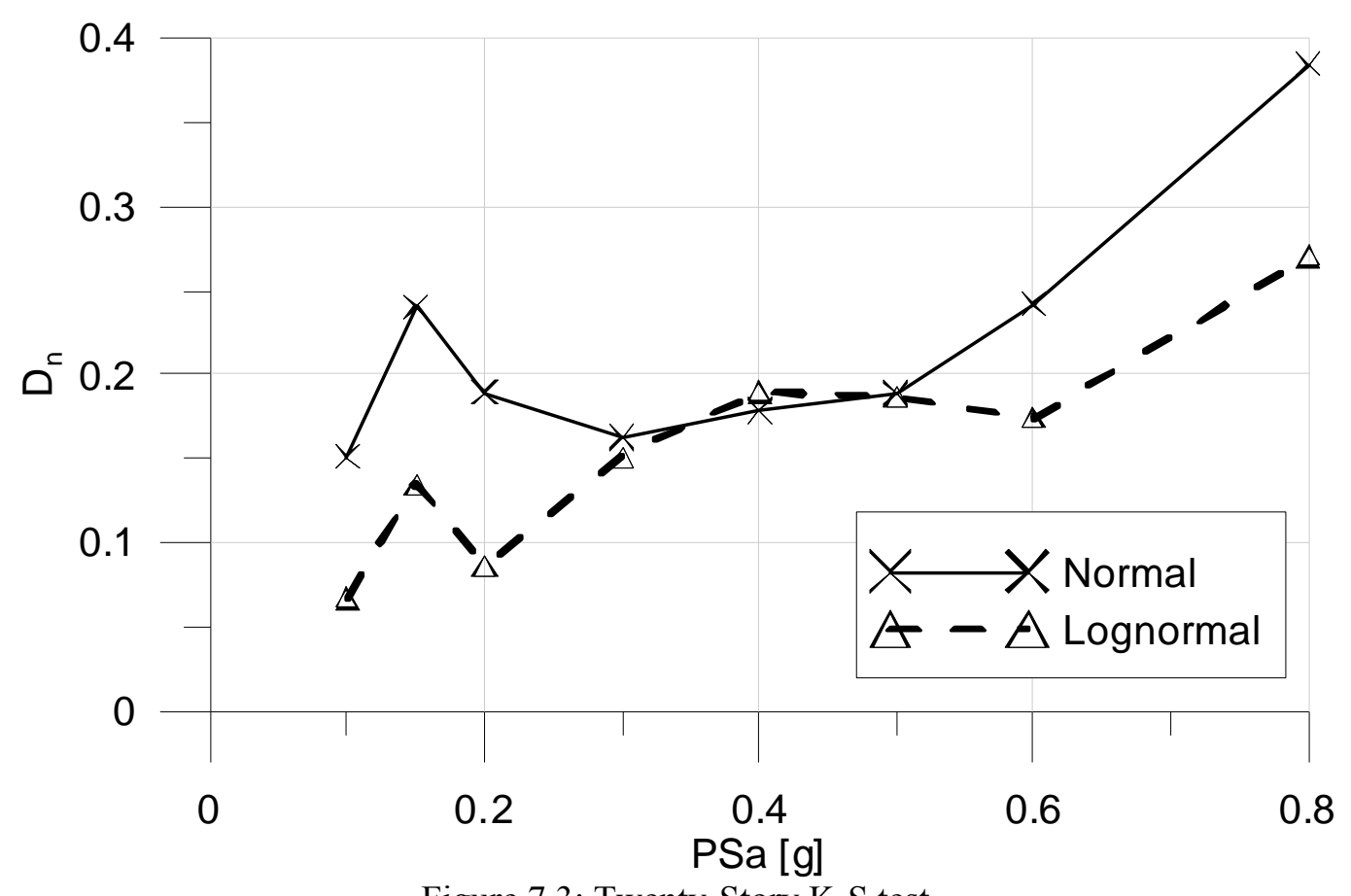

Figure 7.3: Twenty-Story K-S test

\subsection{Fragility Analysis}

A fragility analysis quantifies how a particular parameter is affected over a range of ground motion intensities. For this research the parameter of interest is the maximum observed inter-story drift, and the ground motion intensity measure is the Pseudo-Spectral acceleration (PSa) at the fundamental period of the building. At each level of intensity the probability of the maximum inter-story drift exceeding a given value or drift limit is determined from the distribution of the simulation drifts. Greater detail of fragility analyses can be found in Chang et al. (2009).

Two different fragility analysis methods are applied to the data. The first analysis employs a regression analysis to create smooth curves and the second analysis is conducted on the raw data without the regression analysis. These two methods are compared to evaluate the regression 
analysis assumptions. For both cases, the probabilities of failure are for an assumed level of ground shaking intensity and do not represent the inherent variability of ground shaking.

\subsubsection{Fragility Analysis: Smooth/ Regression Analysis}

The first fragility analysis preformed follows the procedure outlined in Chang et al. (2009) which uses a regression analysis of the drifts to develop smooth fragility curves. However, for this study, PSa is used as the intensity measure instead of Peak Ground Acceleration (PGA). The modified procedure is described below with equations 7.1 and 7.2. The first regression analysis was performed on the maximum drifts to determine the correlation between inter-story drift, $\theta$, and PSa intensity.

$\theta=a(P S a)^{b}$

$P_{f}(\theta>\phi \mid P S a=x)=1-\phi\left(\frac{\ln \left|\frac{\phi}{a x^{b}}\right|}{\beta_{\frac{\theta}{P S a}}}\right)$

A second regression analysis was performed on the standard deviations of the logarithmic drifts to determine $\beta_{\theta / \mathrm{PSa}}$ at each level of intensity. The standard deviations for the regression analysis are calculated from the lognormal distributions of maximum drifts for each earthquake ground motion used. The drift limit, $\varphi$, is defined in FEMA 356 for three limit states: Immediate Occupancy, Life Safety, and Collapse Prevention. Table C1-3 of FEMA 356 defines the structural performance limit drifts for a steel moment resisting frame as $0.7 \%, 2.5 \%$, and $5.0 \%$ for 
Immediate Occupancy, Life Safety, and Collapse Prevention respectively (FEMA 356, 2000, p. $1-14)$.

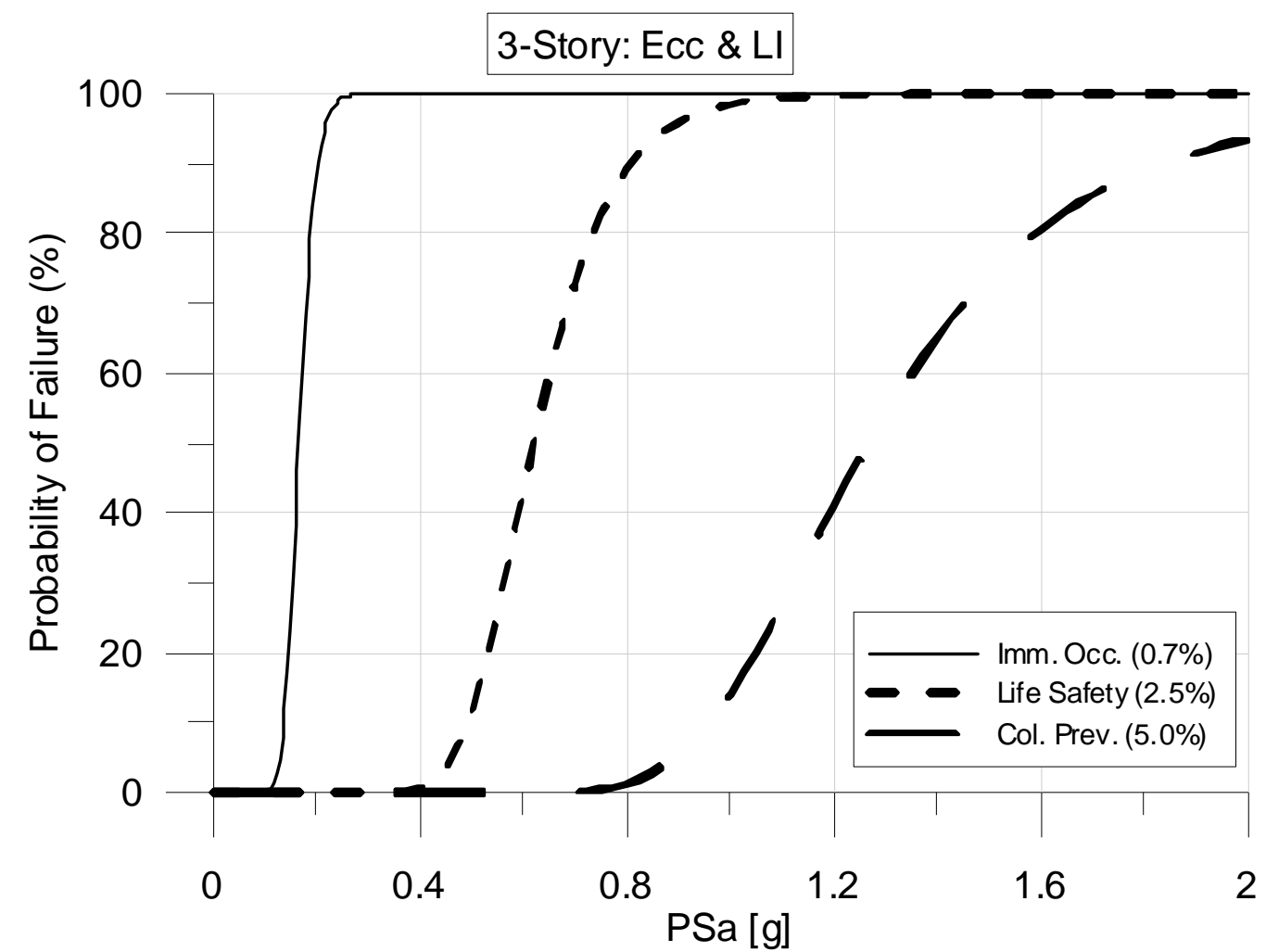

Figure 7.4: Regression fragility curves for load eccentricity and intensity, 3-story

Fig. 7.4, is an example of the smooth/regression fragility curve for the three-story model evaluated with the combined effects of load eccentricity and intensity.

\subsubsection{Fragility Analysis: Rough/ Non-Regression Analysis}

The second set of fragility analyses was performed on the same set of data without using the regression curves. For this rough fragility analysis the drift at each level of ground shaking intensity was taken as the median drift from the set of ground motions. The standard deviation was taken as the average of the individual standard deviations corresponding to each maximum drift value. These individual standard deviations were calculated from the Rosenblueth " $2 \mathrm{k}+1$ " 
analysis and represent the distribution associated with one particular ground motion at one level of ground shaking, given the possible loading scenarios.

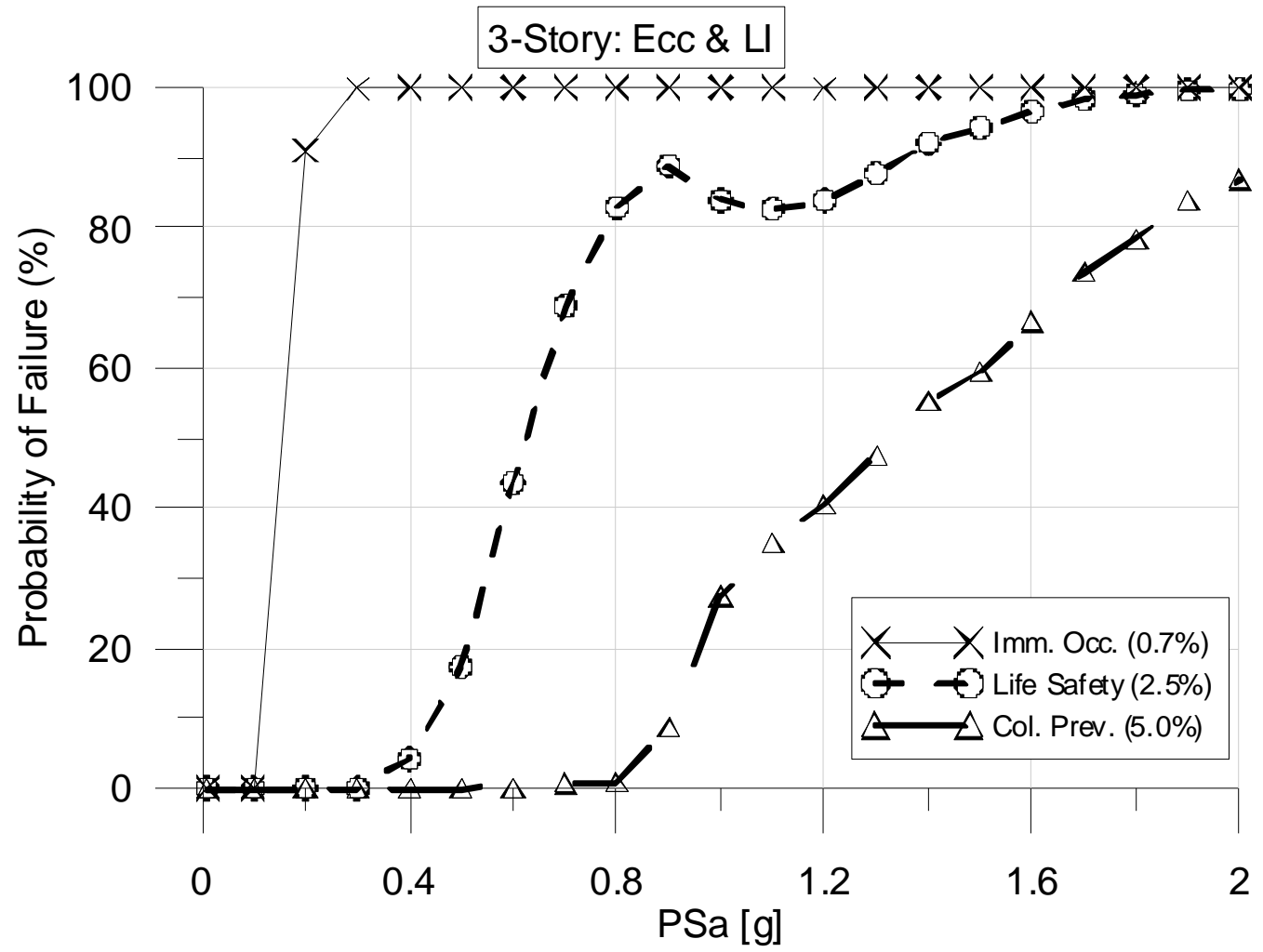

Figure 7.5: Non-regression fragility curves for load eccentricity and intensity, 3-story

Fig. 7.5 shows an example of the rough/non-regression fragility curve for the three-story model evaluated with the combined effects of load eccentricity and intensity. Each point along the curve is based on the variability at each level of intensity and therefore independent from every other point and intensity, which is why the curves are not smooth.

\subsubsection{Fragility Analysis: Comparison}

The two different fragility curves, the smooth/regression and rough/non-regression, show similar results. The regression analysis fragility curves may blend the variability between levels of intensity, but this does not mask the overall results since the smooth and the rough fragility 
curves match up well. This similarity between fragility analyses is shown directly in Fig. 7.6 below.

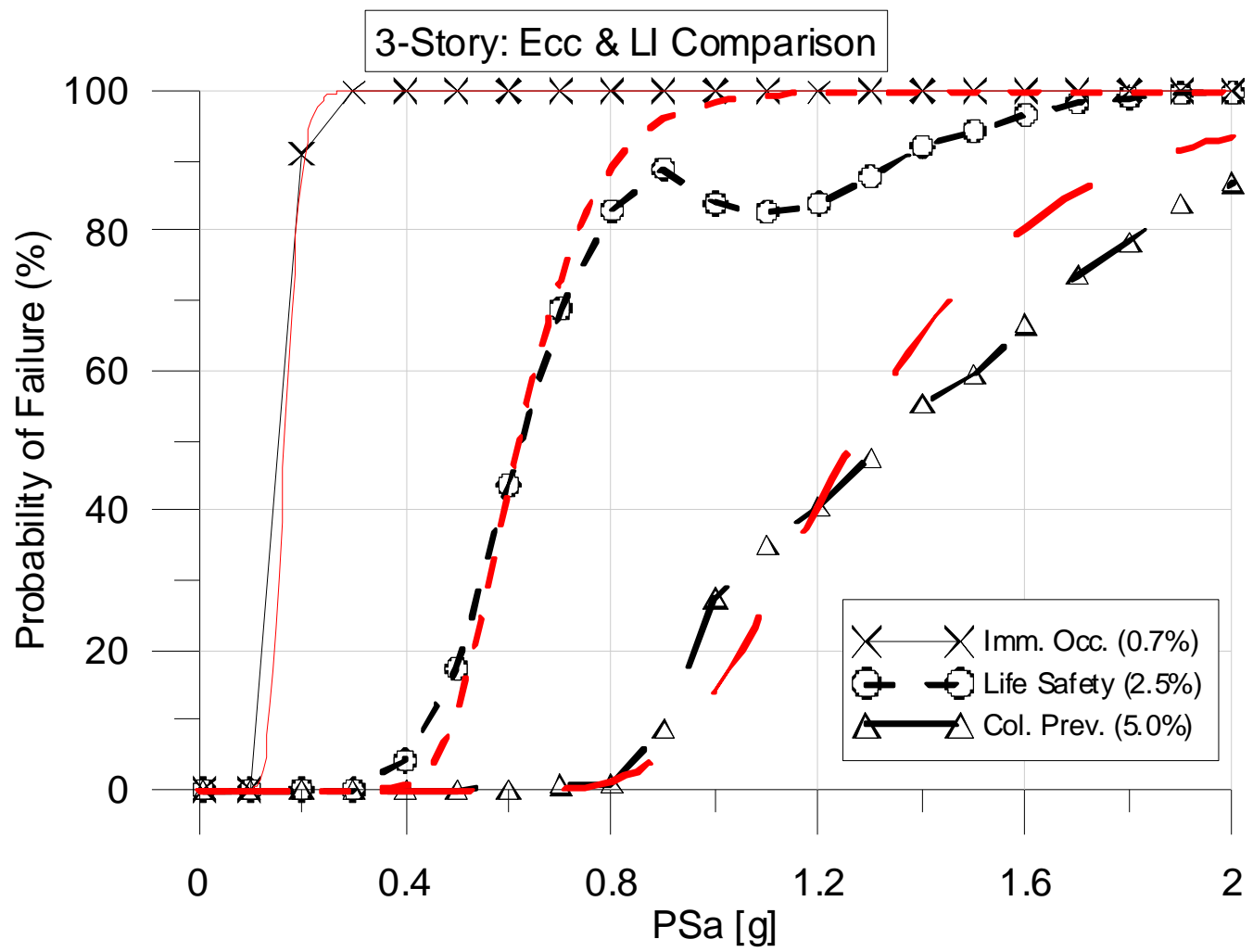

Figure 7.6: Regression and non-regression fragility curve comparison, 3-Story

Since both the rough and smooth curves are nearly identical, the comparison to evaluate the code applied eccentricity results can be made using the smooth curves from the regression analysis. Since the code applied eccentricities were applied with non-integer spectral accelerations the comparison has to be interpolated from the results using the regression analysis. Since there was no Rosenblueth " $2 \mathrm{k}+1$ " Simulation for the code eccentricity scenario, there is also no standard deviation associated with each individual ground motion applied. Rather, the standard deviations are derived from the results of all the applied ground motions. This is identical to how the standard deviations are determined with the regression analysis and will allow for the code values to be compared directly to the Rosenblueth " $2 \mathrm{k}+1$ " Simulation results. Code comparisons are explained in greater detail in sections 7.5 and 7.6. 


\subsection{Fragility Results}

The fragility curves are presented for each model separately. Within each model there are different fragility plots that correspond to each drift limit state. And within these plots there are several curves that represent the various loading conditions.

\subsubsection{Fragility Results: Three-Story}

The three figures below, Figs. 7.7, 7.8, and 7.9, show the fragility curve comparisons for the three limit states: immediate occupancy, life safety, and collapse prevention. Within each of the three plots the fragility curves for each of the different loading scenarios are compared. There are four different loading scenarios: mean (Mean), load intensity only (LI), load eccentricity only (Ecc), and load eccentricity and intensity (Ecc \& LI). Mean refers to all of the variables applied at their respective mean values. Load intensity only refers to the scenario where the load intensity is the only variable being considered while the load eccentricity is held at the mean value. And load eccentricity only refers to the scenario where the load eccentricity is the only variable being considered while the load intensity is held at the mean value. Finally, load eccentricity and intensity represents the case where both the location and intensity of the load are treated as variables. 


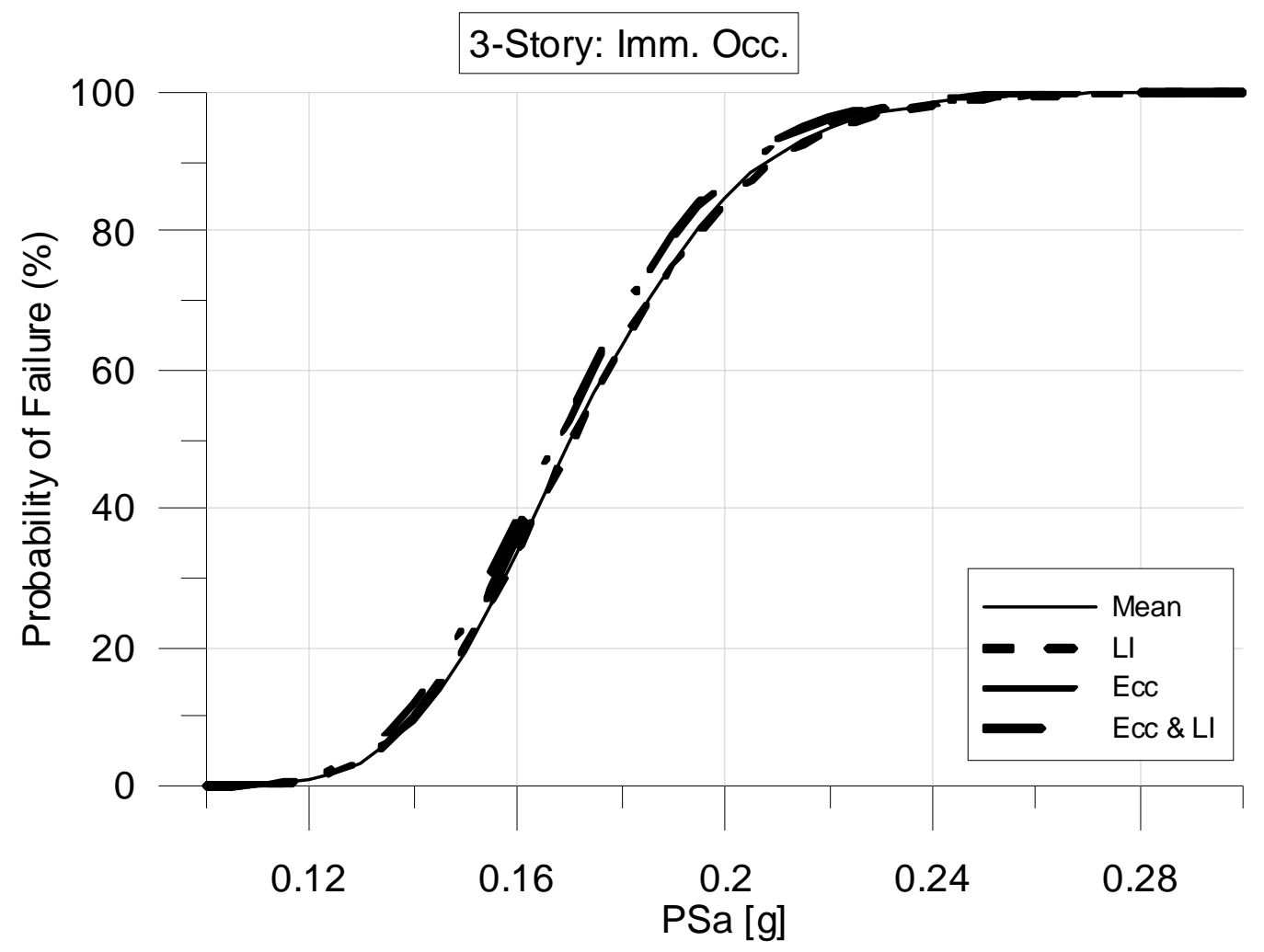

Figure 7.7: Regression fragility curves for immediate occupancy comparison, 3-story

It can be seen from Fig. 7.7 above that the first limit state, immediate occupancy ( $0.7 \%$ drift), is little affected by the loading scenario. All of the fragility curves are essentially aligned with one another, although the two eccentric loading scenarios, "Ecc" and "Ecc \& LI", are slightly more susceptible than the load intensity only and mean scenarios, and experience slightly greater drifts. These differences are mainly quantitative and are numerically shown in Table 7.3: Probability of Exceeding Immediate Occupancy Drift Limit. 


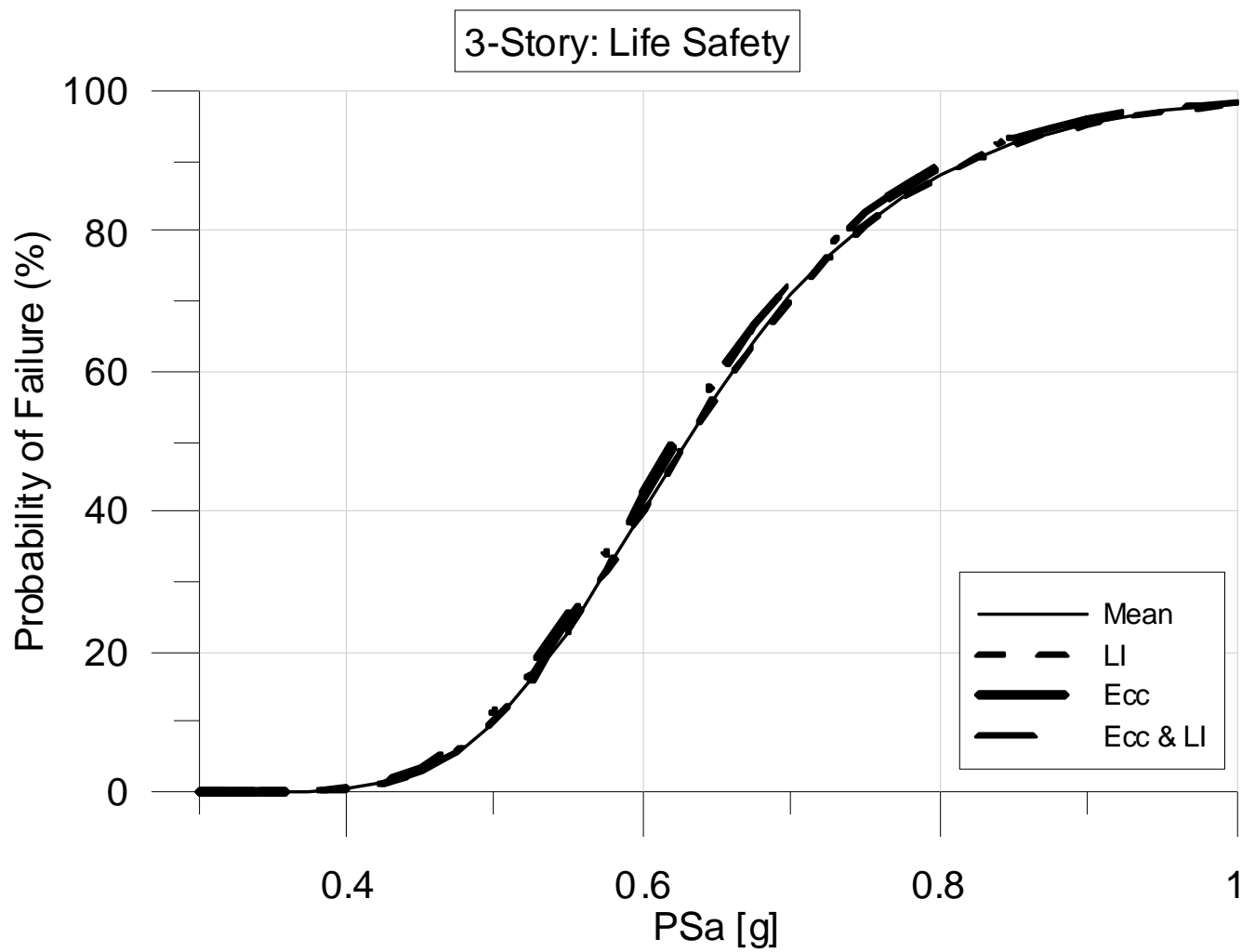

Figure 7.8: Regression fragility curves for life safety comparison, 3-story

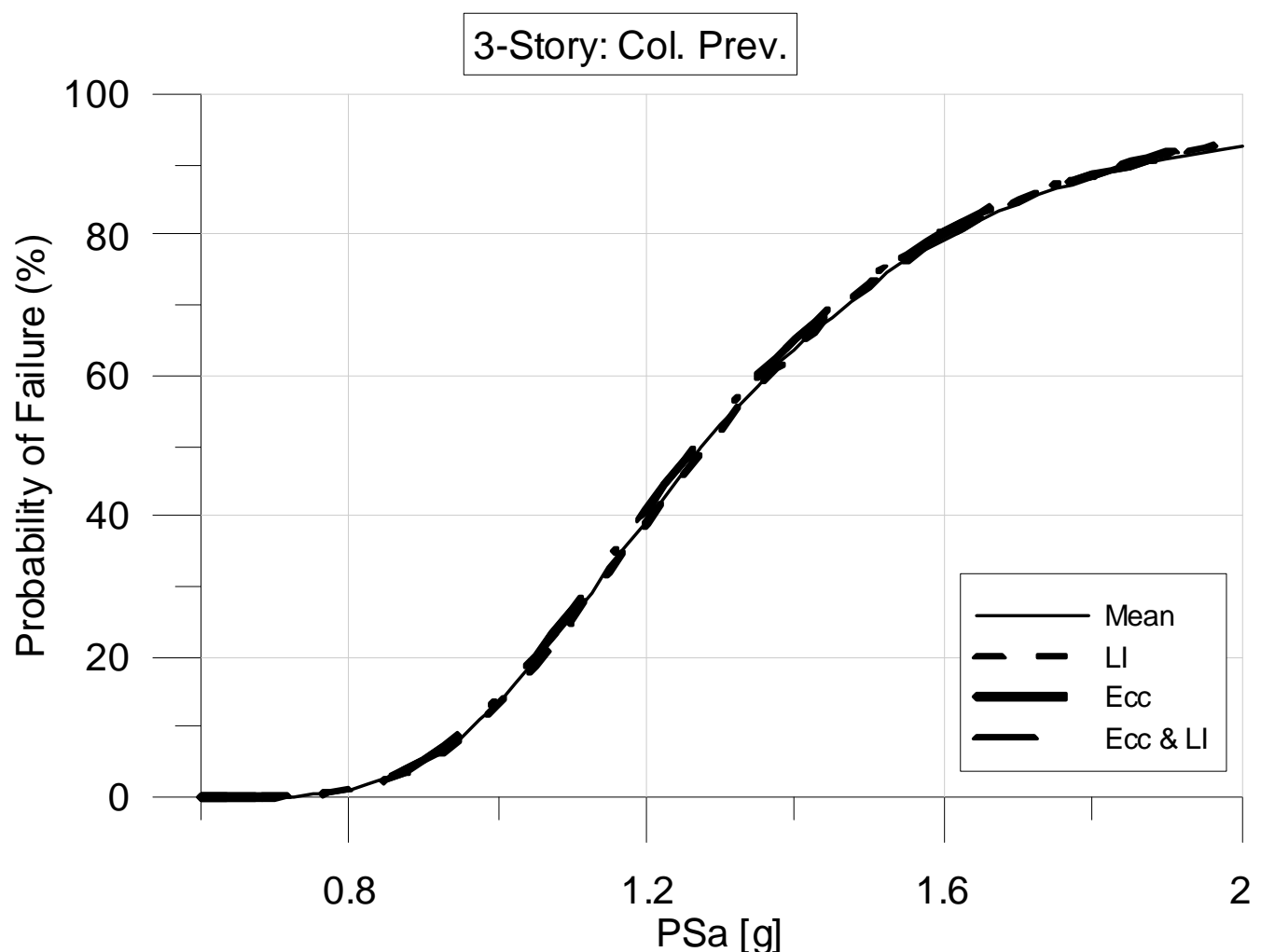

Figure 7.9: Regression fragility curves for collapse prevention comparison, 3-story 
As with the first limit state, the next two, life safety and collapse prevention, also show minimal/ negligible difference between the different loading scenarios. Therefore, the three-story building is predominantly governed by the intensity of the ground shaking and is negligibly affected by the variation of mass location and intensity given the assumed predefined distributions.

\subsubsection{Fragility Results: Nine-Story}

The limit state fragility curves for the nine-story model are compared for the various loading scenarios in the three figures below, Figs. 7.10, 7.11, and 7.12. Unlike the response of the threestory building, the nine-story building is noticeably affected by the various loading scenarios. The

difference seen between fragility curves within a particular limit state becomes more evident at greater drifts. While at the lower level drift for immediate occupancy the fragility curves are essentially aligned, Fig. 7.10 below illustrates the co-alignment of the curves. 


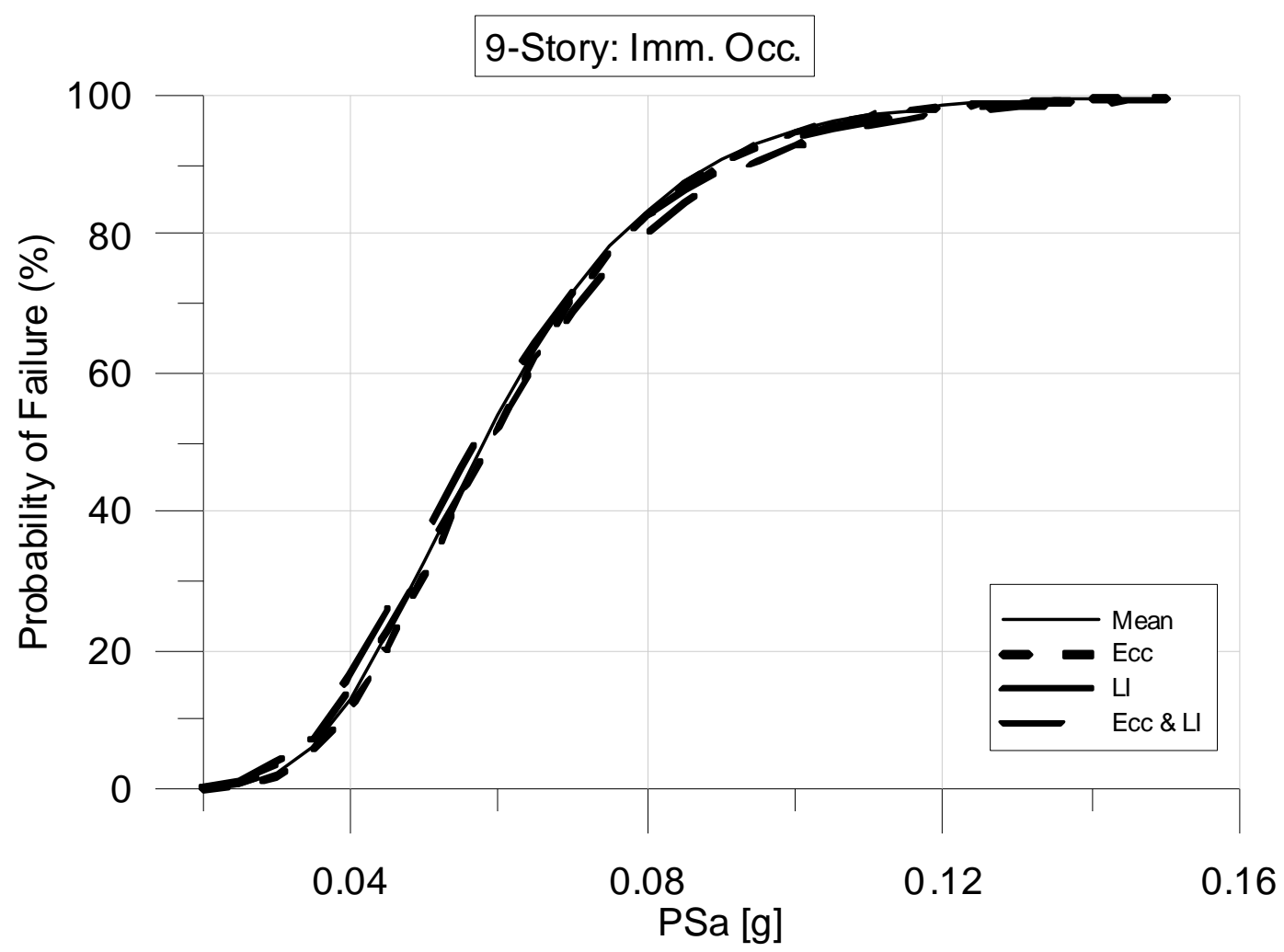

Figure 7.10: Regression fragility curves for immediate occupancy comparison, 9-story

Moving onto the life safety and collapse prevention drift limit states, the effects of the different loading scenarios becomes evident. The scenario that considers both load eccentricity and intensity, "Ecc \& LI", has greater drifts, which shift the fragility curve to the left resulting in greater probabilities of failure at a given level of intensity. The load eccentricity only scenario, "Ecc", results in the next largest drift followed by load intensity only, "LI", and mean. For collapse prevention, the increased drifts result in either a ten percent difference of failure probability given a likely level of ground motion intensity, or a difference of $0.1 \mathrm{~g}$ PSa given a desired probability of failure 


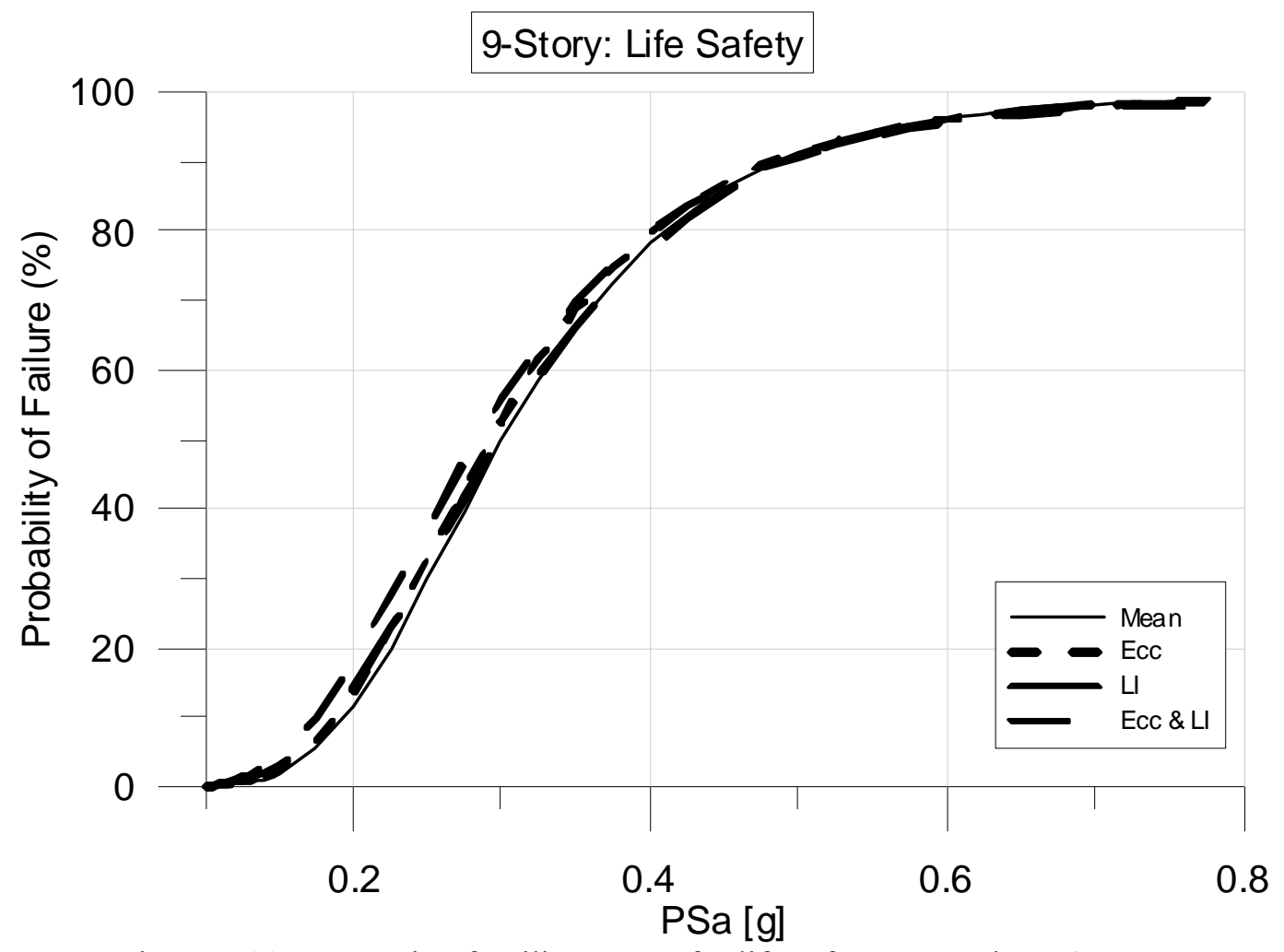

Figure 7.11: Regression fragility curves for life safety comparison, 9-story

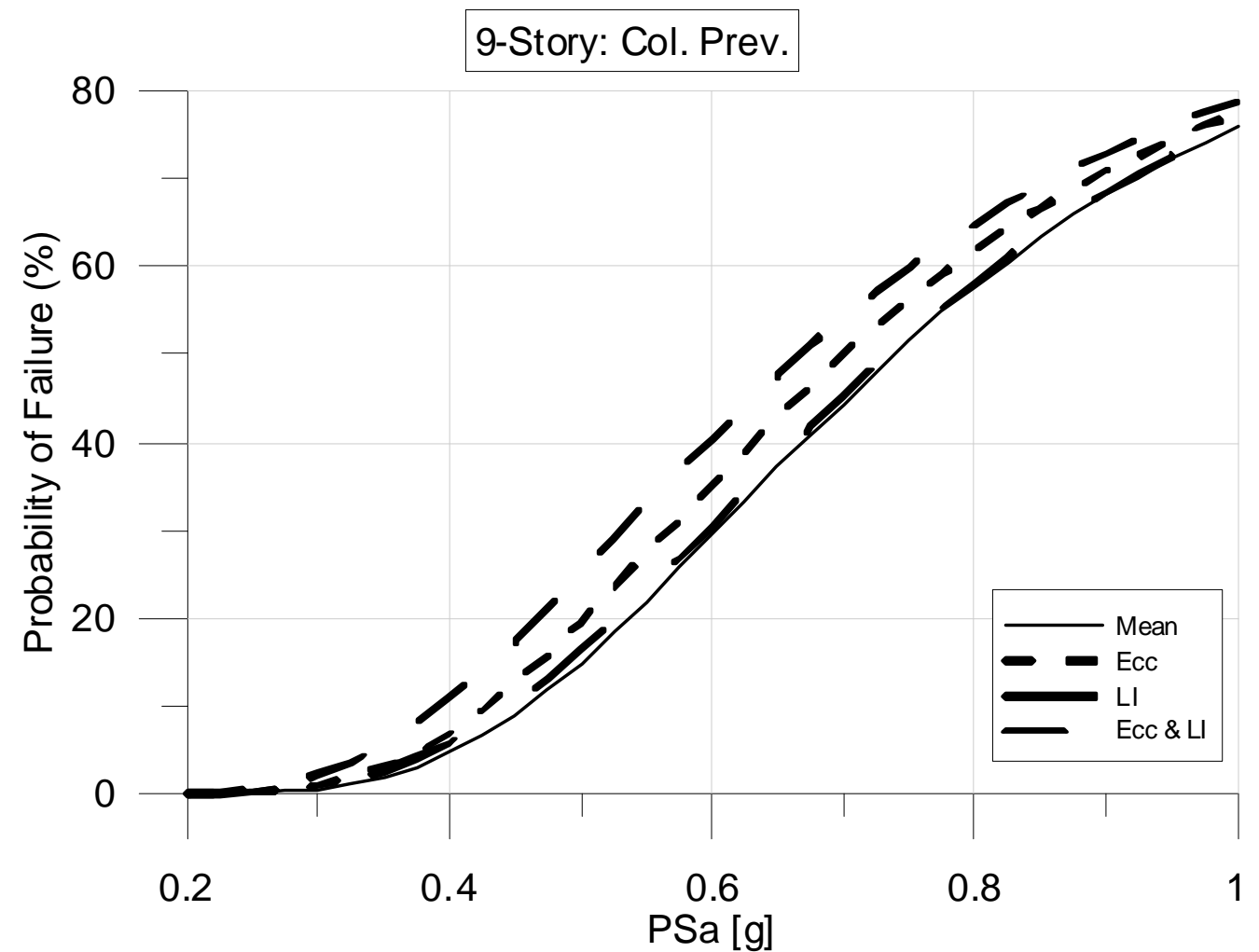

Figure 7.12: Regression fragility curves for collapse prevention comparison, 9-story 


\subsubsection{Fragility Results: Twenty-Story}

The limit state fragility curves for the twenty-story model are compared for the various loading scenarios in the three figures below, Figs. 7.13, 7.14, and 7.15. Similar to the results observed from the three-story building, the twenty-story building is minimally to negligibly affected by the different loading scenarios. The fragility curves within each limit state are essentially co-aligned showing that the effects of mass eccentricity and intensity have minimal impact on the drifts for the twenty story building.

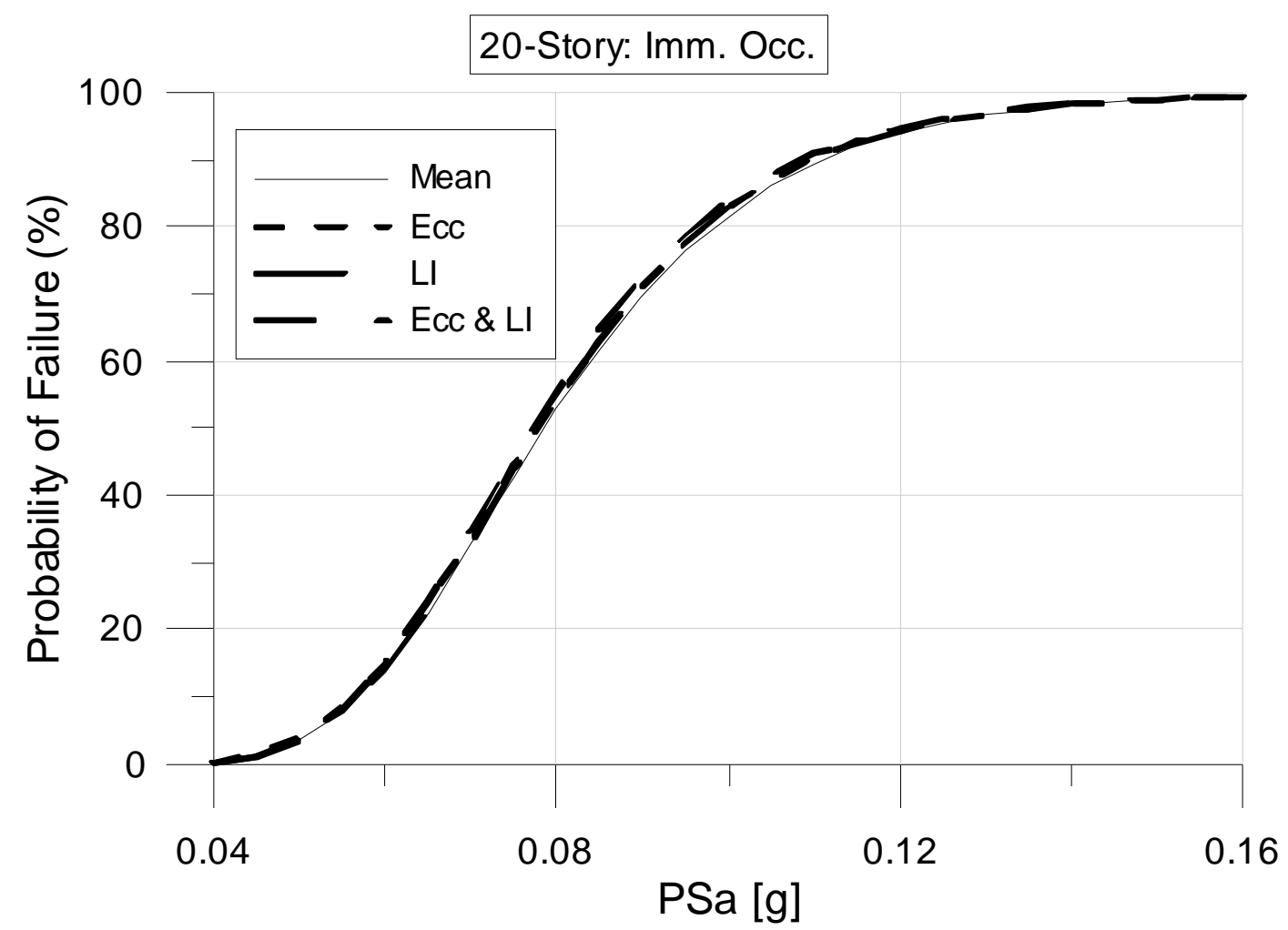

Figure 7.13: Regression fragility curves for immediate occupancy comparison, 20-story 


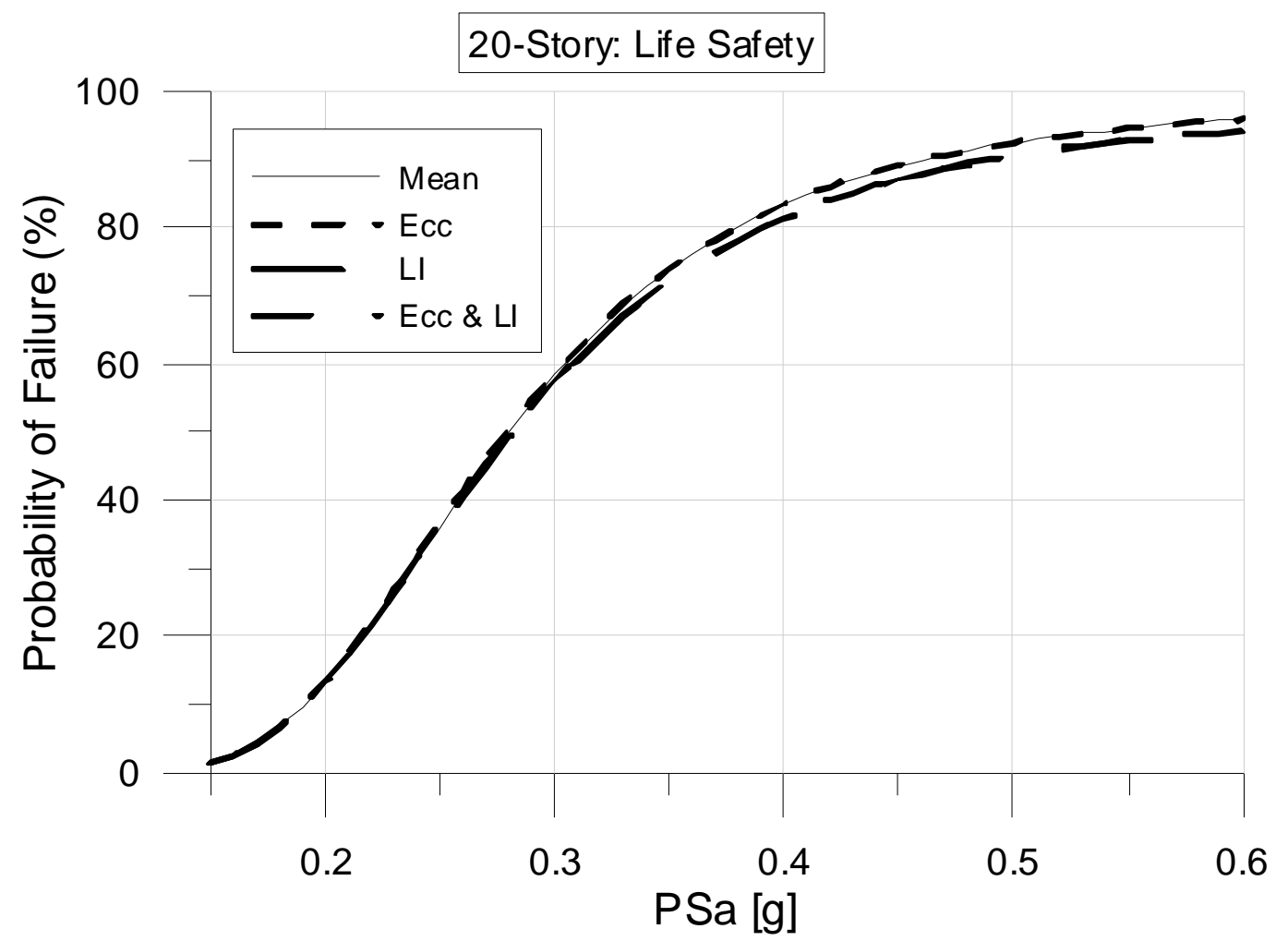

Figure 7.14: Regression fragility curves for life safety comparison, 20-story

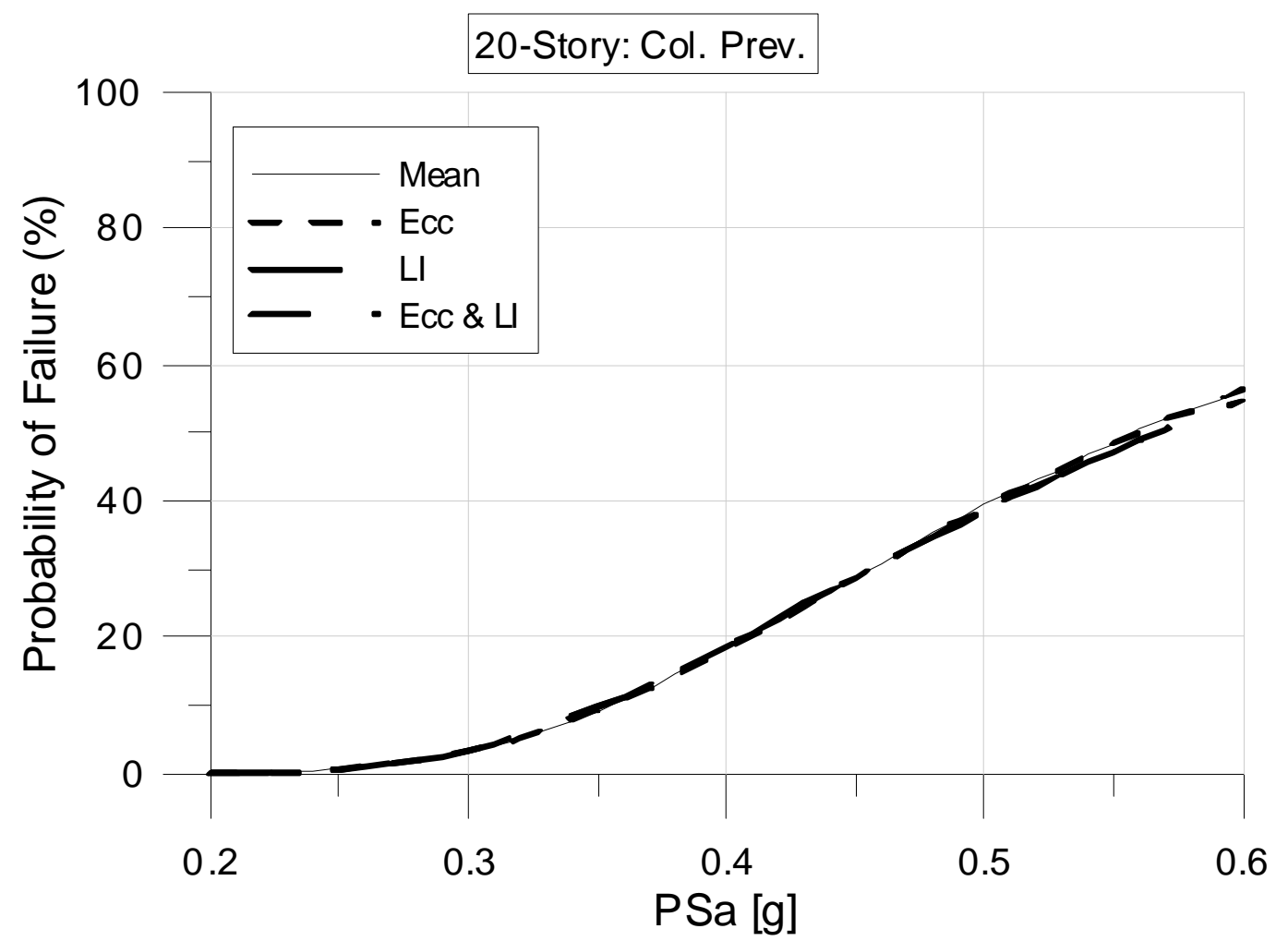

Figure 7.15: Regression fragility curves for collapse prevention comparison, 20-story 


\subsection{Collapse Margin Ratio}

The fragility curves represent the probability of a particular loading scenario exceeding a limit state over a range of intensities. They can also be used to evaluate the collapse safety at a particular intensity. The Collapse Margin Ratio, CMR, is a measure of collapse safety. It is presented in FEMA P695: Quantification of Building Seismic Performance Factors and is defined as the ratio of the spectral acceleration at which $50.0 \%$ of the ground motions cause collapse to the spectral acceleration of the maximum considered earthquake, MCE, spectrum at the fundamental period of the structure (FEMA P-695, 2009). When the ratio is greater than one, the MCE has less than $50.0 \%$ chance of collapsing the structure. Table 7.1 lists the CMR for each model at each loading scenario. The first row, $\mathrm{S}_{(\mathrm{MT})}$, is the spectral acceleration of the MCE response spectra at the fundamental period of each building. Each of the next consecutive rows, $\mathrm{S}_{\left(\mathrm{CT}, \_\right.}$, is the spectral acceleration with a $50.0 \%$ probability of failure for the various loading scenarios.

Table 7.1: Collapse margin ratios, CMR

\begin{tabular}{|c|c|c|c|c|c|c|}
\hline \multirow{2}{*}{$\begin{array}{l}\text { Collapse } \\
\text { Margin Ratio }\end{array}$} & \multicolumn{2}{|c|}{ 3-Story } & \multicolumn{2}{|c|}{ 9-Story } & \multicolumn{2}{|c|}{ 20-Story } \\
\hline & $\mathrm{S}_{\mathrm{a}}(\mathrm{g})$ & CMR & $\mathrm{Sa}_{\mathrm{a}}(\mathrm{g})$ & CMR & $\mathrm{Sa}(\mathrm{g})$ & $\mathrm{CMR}$ \\
\hline $\mathrm{S}(\mathrm{MT})$ & 1.244 & ----- & 0.547 & ----- & 0.316 & ----- \\
\hline $\mathrm{S}_{(\mathrm{CT}, \mathrm{Ecc} \& \mathrm{LI})}$ & 1.269 & 1.020 & 0.668 & 1.221 & 0.568 & 1.797 \\
\hline $\mathrm{S}_{(\mathrm{CT}, \mathrm{Ecc})}$ & 1.269 & 1.020 & 0.701 & 1.282 & 0.560 & 1.772 \\
\hline $\mathrm{S}(\mathrm{CT}, \mathrm{LI})$ & 1.280 & 1.029 & 0.736 & 1.346 & 0.568 & 1.797 \\
\hline $\mathrm{S}_{(\mathrm{CT}, \text { Mean })}$ & 1.280 & 1.029 & 0.740 & 1.353 & 0.559 & 1.769 \\
\hline
\end{tabular}

All of the CMRs are greater than one, showing that regardless of which loading scenario is considered, the buildings will not have a probability of greater than $50 \%$ to collapse under MCE events. As expected from the fragility curves the CMR values for the three-story and twenty-story models are all constant. The CMR values for the nine-story model, however, range depending on loading considerations. Consideration of mass eccentricity and mass intensity results in 
significantly less conservative results compared to the mean loading case. Moreover, looking at the CMRs for each of the three buildings, the ratios increase with each consecutive building, which means that as the building height increases the chance of collapse decreases.

\subsection{Evaluation of Code Provisions for Consideration of Reactive Weight Eccentricity}

To evaluate the code defined eccentricities, two specific analyses were run for each model. Both analyses were run with the same code applied eccentricity, defined as a 5.0\% mass eccentricity applied along both plan dimensions at each floor level, and the intensities of the floor masses were all applied at their respective mean values. All of the eccentricities were applied in the same directions at each floor level. The first analysis was run with the ground motions scaled to the design based earthquake intensity, DBE, and the second with a maximum considered earthquake intensity, MCE. A design response spectrum for the Los Angeles area was developed using the USGS website in accordance with ASCE 7-10 specifications, the USGS report is in Appendix C. All of the ground motions were scaled to the spectral acceleration determined from the design response spectra at the fundamental period of each model for the DBE intensity. These scaling factors were then multiplied by 1.5 to represent the MCE intensity.

These scenarios were run for each model and the maximum inter-story drifts for each ground motion were used to represent the drift distribution of code applied eccentricities.. These distributions were then compared to the distributions for the various loading scenarios for each model. Table 7.2 lists the ratio of the expected code based drifts to the various loading scenario Rosenblueth " $2 \mathrm{k}+1$ " Simulation drifts. Ratios that are greater than 1.0 show conservatism of the code prediction, and values less than 1.0 show an underestimation by the code. As expected from the fragility plots for the three-story and twenty-story models the ratios of the code drift to any of the loading scenario drifts is essentially constant regardless of the loading variables that are 
considered. The nine-story model is noticeably affected by the various loading scenarios and experiences significant increase in drift from the mean loading scenario.

Table 7.2: Code drift normalized by Rosenblueth " $2 \mathrm{k}+1$ ” drift

\begin{tabular}{|c|c|c|c|c|c|}
\hline \multirow[b]{2}{*}{ Model } & \multirow[b]{2}{*}{ Design Event } & \multicolumn{4}{|c|}{ Code/Rosenblueth Simulation } \\
\hline & & Ecc \& LI & Ecc & LI & Mean \\
\hline \multirow[t]{2}{*}{3} & DBE & 0.980 & 0.981 & 0.991 & 0.990 \\
\hline & MCE & 1.036 & 1.037 & 1.047 & 1.045 \\
\hline \multirow[t]{2}{*}{9} & DBE & 0.911 & 0.938 & 0.958 & 0.964 \\
\hline & MCE & 0.979 & 1.015 & 1.047 & 1.052 \\
\hline \multirow[t]{2}{*}{20} & DBE & 1.228 & 1.222 & 1.233 & 1.227 \\
\hline & MCE & 1.199 & 1.189 & 1.202 & 1.192 \\
\hline
\end{tabular}

*Values greater than 1.0 show conservatism of the code

The code drifts for the three-story building are right around the expected drifts. The code is slightly un-conservative at the DBE level and slightly conservative at the MCE level. The drifts of the nine-story building have not been conservatively captured by the code. At the DBE level the consideration of both mass intensity and location of the mass results in drifts that are about 9.0\% greater than that of the code. At the MCE level the drifts range from conservative to unconservative depending on which loading scenarios are considered. When the mass intensity and location are considered independently, the code drifts are on the conservative side. However, when both of these variables are considered at the same time, the code drifts become unconservative. Like the three-story building, the twenty-story building was little affected by the various loading scenarios. However, for the twenty-story building the code drifts are conservative for each loading scenario at each level of ground shaking: DBE and MCE. On average the drifts are over estimated by about $20.0 \%$. 


\subsection{Building Performances under DBE and MCE Events}

The three tables below, Tables 7.3, 7.4, and 7.5, list the probability that each of the various loading scenarios exceeds one of the three limit states. The conservatism of the code is demonstrated when the code probability is greater than one of the other loading scenario probabilities. This numerically represents the fragility curve data at specific intensities, DBE and MCE. This is done for a direct comparison to the code values because the code analysis was applied as a discrete analysis at two intensities rather than as an incremental dynamic analysis.

Table 7.3: Probability of exceeding immediate occupancy drift limit

\begin{tabular}{|c|c|c|c|c|c|c|}
\hline \multirow[b]{2}{*}{ Model } & \multirow[b]{2}{*}{$\begin{array}{l}\text { Design } \\
\text { Event }\end{array}$} & \multicolumn{5}{|c|}{ Immediate Occupancy: 0.7\% Drift Limit } \\
\hline & & $\begin{array}{l}\text { Ecc \& } \\
\text { LI }\end{array}$ & Ecc & LI & Mean & Code \\
\hline \multirow[t]{2}{*}{3} & DBE & $100.0 \%$ & $100.0 \%$ & $100.0 \%$ & $100.0 \%$ & $100.0 \%$ \\
\hline & MCE & $100.0 \%$ & $100.0 \%$ & $100.0 \%$ & $100.0 \%$ & $100.0 \%$ \\
\hline \multirow[t]{2}{*}{9} & DBE & $100.0 \%$ & $100.0 \%$ & $100.0 \%$ & $100.0 \%$ & $100.0 \%$ \\
\hline & MCE & $100.0 \%$ & $100.0 \%$ & $100.0 \%$ & $100.0 \%$ & $100.0 \%$ \\
\hline \multirow[t]{2}{*}{20} & $\mathrm{DBE}$ & $100.0 \%$ & $100.0 \%$ & $100.0 \%$ & $100.0 \%$ & $100.0 \%$ \\
\hline & $\mathrm{MCE}$ & $100.0 \%$ & $100.0 \%$ & $100.0 \%$ & $100.0 \%$ & $100.0 \%$ \\
\hline
\end{tabular}

Table 7.4: Probability of exceeding life safety drift limit

\begin{tabular}{|c|c|c|c|c|c|c|}
\hline \multirow[b]{2}{*}{ Model } & \multirow[b]{2}{*}{$\begin{array}{l}\text { Design } \\
\text { Event }\end{array}$} & \multicolumn{5}{|c|}{ Life Safety: $2.5 \%$ Drift Limit } \\
\hline & & $\begin{array}{l}\text { Ecc \& } \\
\text { LI }\end{array}$ & Ecc & LI & Mean & Code \\
\hline \multirow[t]{2}{*}{3} & DBE & $91.5 \%$ & $91.6 \%$ & $90.5 \%$ & $90.5 \%$ & $85.8 \%$ \\
\hline & MCE & $99.8 \%$ & $99.8 \%$ & $99.8 \%$ & $99.8 \%$ & $99.7 \%$ \\
\hline \multirow[t]{2}{*}{9} & DBE & $73.2 \%$ & $72.6 \%$ & $70.2 \%$ & $70.2 \%$ & $65.2 \%$ \\
\hline & MCE & $93.5 \%$ & $94.1 \%$ & $93.7 \%$ & $94.2 \%$ & $97.1 \%$ \\
\hline \multirow[t]{2}{*}{20} & DBE & $31.2 \%$ & $31.6 \%$ & $30.9 \%$ & $31.2 \%$ & $57.1 \%$ \\
\hline & MCE & $62.9 \%$ & $64.4 \%$ & $62.6 \%$ & $64.1 \%$ & $84.6 \%$ \\
\hline
\end{tabular}


Table 7.5: Probability of exceeding collapse prevention drift limit

\begin{tabular}{|c|c|c|c|c|c|c|}
\hline \multirow[b]{2}{*}{ Model } & \multirow[b]{2}{*}{$\begin{array}{l}\text { Design } \\
\text { Event }\end{array}$} & \multicolumn{5}{|c|}{ Collapse Prevention: 5.0\% Drift Limit } \\
\hline & & $\begin{array}{l}\text { Ecc \& } \\
\text { LI } \\
\end{array}$ & Ecc & LI & Mean & Code \\
\hline \multirow[t]{2}{*}{3} & DBE & $1.9 \%$ & $1.8 \%$ & $1.7 \%$ & $1.7 \%$ & $3.3 \%$ \\
\hline & $\mathrm{MCE}$ & $46.8 \%$ & $46.8 \%$ & $45.2 \%$ & $45.6 \%$ & $43.6 \%$ \\
\hline \multirow[t]{2}{*}{9} & DBE & $6.9 \%$ & $4.0 \%$ & $3.4 \%$ & $2.6 \%$ & $2.1 \%$ \\
\hline & MCE & $32.3 \%$ & $26.8 \%$ & $22.7 \%$ & $21.5 \%$ & $25.1 \%$ \\
\hline \multirow[t]{2}{*}{20} & DBE & $0.4 \%$ & $0.4 \%$ & $0.4 \%$ & $0.4 \%$ & $1.0 \%$ \\
\hline & MCE & $5.1 \%$ & $4.8 \%$ & $5.1 \%$ & $4.8 \%$ & $8.7 \%$ \\
\hline
\end{tabular}

The code results show that the drifts are not necessarily conservatively captured for the threestory and nine-story buildings. The combined effects of mass eccentricity and intensity can result in expected drifts that are larger than the code drifts. This is most evident for the nine-story building, but the CMR values from Section 7.4 show that all of the buildings are adequately protected against collapse. This protection against collapse occurs despite the code's ability to capture the drifts generated from the various loading scenarios. Therefore, although the code is not always conservative it is not so un-conservative as to cause concern for collapse.

\subsection{Performance Based Evaluation: FEMA 350}

In addition to the fragility analyses, the drift data has also been evaluated using the procedure outlined in FEMA 350 Chapter 4: Performance Evaluation and Appendix A: Detailed Procedures for Performance Evaluation (FEMA 350, 2000). This procedure estimates the likelihood of a performance objective being achieved with a confidence level. The procedure is based on the results of generic buildings and consequently the results are expected to have some inherent 
uncertainty. Also this procedure only evaluates the buildings at two structural performance levels:

Immediate Occupancy and Collapse Prevention, defined below:

\section{Immediate Occupancy Performance Level}

The Immediate Occupancy structural performance level is defined as the post-earthquake damage state in which only limited structural damage has occurred. Damage is anticipated to be so slight that it would not be necessary to inspect the building for damage following the earthquake, and such little damage as may be present would not require repair. The basic vertical-and lateral-force-resisting systems of the building retain nearly all of their pre-earthquake strength and stiffness. The risk of lifethreatening injury as a result of structural damage is very low. Buildings meeting this performance level should be safe for immediate post-earthquake occupancy, presuming that damage to nonstructural components is suitably light and that needed utility services are available. (FEMA 350, 2000, p. 4-8)

\section{Collapse Prevention Performance Level}

The Collapse Prevention structural performance level is defined as the post-earthquake damage state in which the structure is on the verge of experiencing partial or total collapse. Substantial damage to the structure has occurred, potentially including significant degradation in the stiffness and strength of the lateral-force-resisting system, large permanent lateral deformation of the structure, and, to a more limited extent, degradation in the vertical-load-carrying capacity. However, all significant components of the gravity-load-resisting system must continue to carry their gravity-load demands. The structure may not be technically or economically practical to repair and is not safe for re-occupancy; aftershock activity could credibly induce collapse. (FEMA 350, 2000, p. 4-8)

The Life Safety performance level is not outlined in this procedure, but it can be evaluated based on individual criteria and by interpolating between Immediate Occupancy and Collapse

Prevention.

The FEMA 350 procedure is based on a group of buildings with a range of configurations and, therefore, is not representative of any specific building. Since the factors and coefficients used in the procedure are based on these buildings, and due to the generality of these buildings, the results have an inherent uncertainty. 


\subsubsection{Determination of Confidence Levels}

Each of the three Los Angeles models was evaluated based solely on inter-story drifts and neglected the provisions for axial column forces. For each intensity, a confidence index parameter is determined. The index parameter is a factored-demand-to-capacity ratio, $\lambda$. The demand is taken as the median maximum inter-story drift determined through the nonlinear dynamic time history analyses. The demand is then factored by variables that account for the modeling assumptions and the variability of ground shaking. The capacity is based on the performance level and type of lateral moment resisting frame: SMF or OMF. The capacity is then reduced by a coefficient to account for the variability associated with the prediction of the structural capacity.

In addition to the factored-demand-to-capacity ratio, an uncertainty parameter, $\beta_{\mathrm{UT}}$, is also required to determine the confidence level at a given level of intensity. The uncertainty parameter attempts to account for all the sources of uncertainty. It is based on performance level, building height, and type of lateral moment resisting frame. With these two values, $\lambda$ and $\beta_{\mathrm{UT}}$, the confidence level can be determined at each intensity from Table A-1 from FEMA 350 (FEMA 350, 2000, p. A-8). The confidence level represents the probability that a particular performance level will not be exceeded. A confidence level of $95 \%$ for a given performance level corresponds to a $5.0 \%$ probability that that particular performance level will be exceeded given the intensity of ground shaking. FEMA 350 suggests that for an Immediate Occupancy performance level a minimum confidence level of 50\% should be employed, and for Collapse Prevention a minimum confidence level of $90 \%$ should be employed (p. 4-35). For comparison purposes the confidence levels were subtracted from a value of one to turn the confidence level plots into fragility curves with a y-axis that now represents the probability of exceeding limit states, probability of failure. The performance based curves can now be directly compared to the fragility curves at the two limit states. For greater detail about the procedure and factors refer to FEMA 350 (2000). 


\subsubsection{P- $\Delta$ Effects}

The models are checked at each story for P- $\Delta$ effects in accordance with Section 2.8.6 of FEMA 350. Equation (2-1) of FEMA 350 calculates the ratio of the overturning gravity moment to the restoring story shear force moment (FEMA 350, 2000, p. 2-17). A ratio greater than 1.0 represents a condition where the structure collapses because it does not have enough strength to resist the P- $\Delta$ effects. For values greater than 0.3 but less than 1.0 the $\mathrm{P}-\Delta$ effects are taken into account with the procedures provided in Appendix A of FEMA 350. Because many of the ratios were greater than 0.3 the procedures of Appendix A were employed for all three models.

\subsubsection{Comparison to Regression Fragility Curves}

The FEMA 350 Performance Based Evaluation method defines the immediate occupancy drift limit as $2.0 \%$ and collapse prevention drift limit as $10.0 \%$. These limits are greater than or equal to twice that of the limits defined in the fragility analysis, $0.7 \%$ and $5.0 \%$ respectively. Therefore the results of this performance-based analysis are expected to be much more un-conservative when compared to the fragility analysis, despite the factored coefficients. Due to the discrepancy between the drift limits the FEMA analysis was also run with reduced capacities of $0.7 \%$ and $5.0 \%$ 


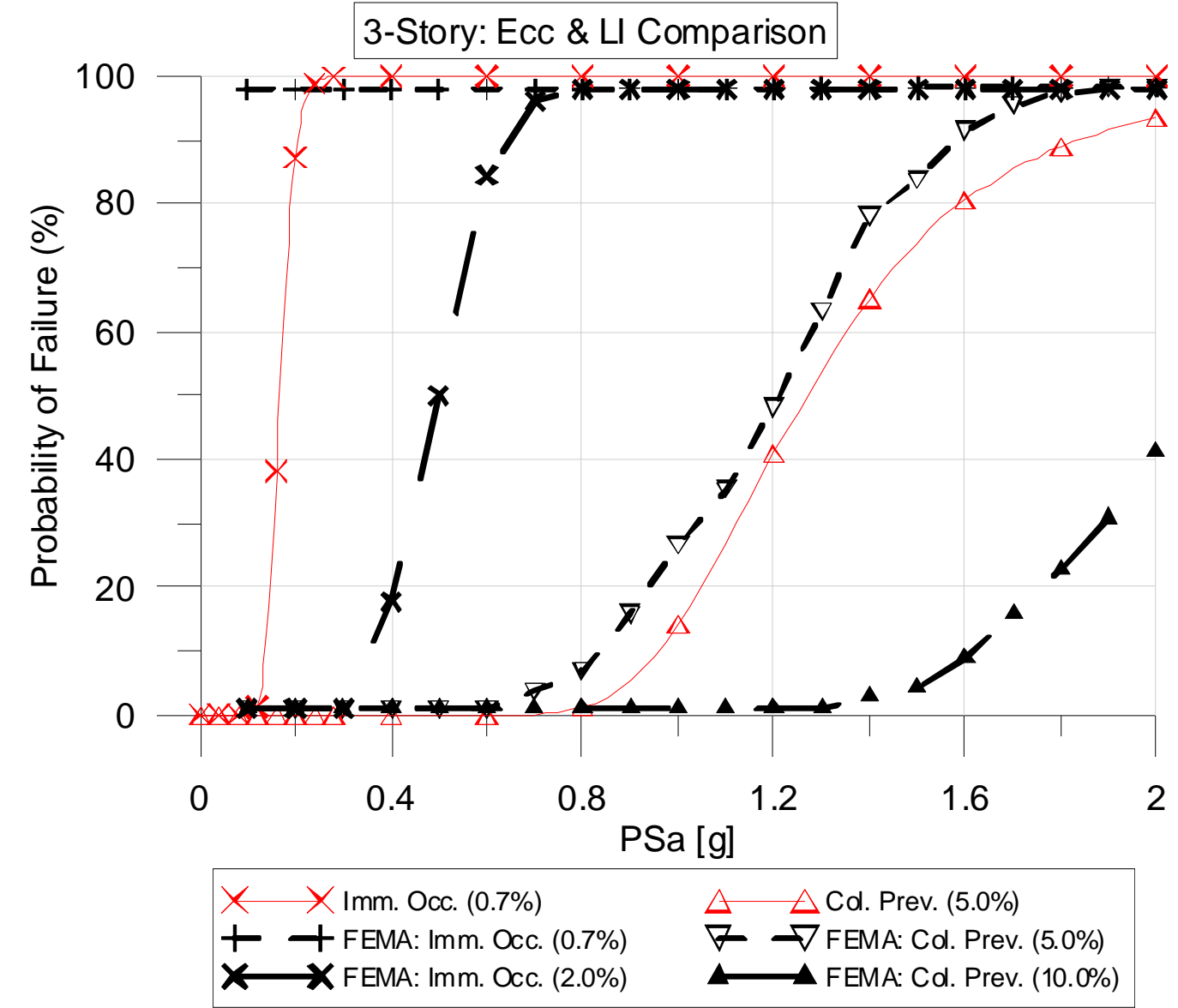

Figure 7.16: FEMA fragility curves, with code capacity and reduced capacity, compared to regression fragility curves for three-story model

Above, Fig. 7.16 directly compares the three-story smooth/regression fragility curves, immediate occupancy and collapse prevention, for mass eccentricity and intensity against the two sets of fragility curves from the FEMA 350 analysis. As expected, the original FEMA analysis with the larger drift capacities produced much more un-conservative results. The second set of fragility curves from the FEMA analysis were performed with reduced drift capacities, to match those from the smooth/regression fragility curves, and produced much more comparable results for the three-story model. 


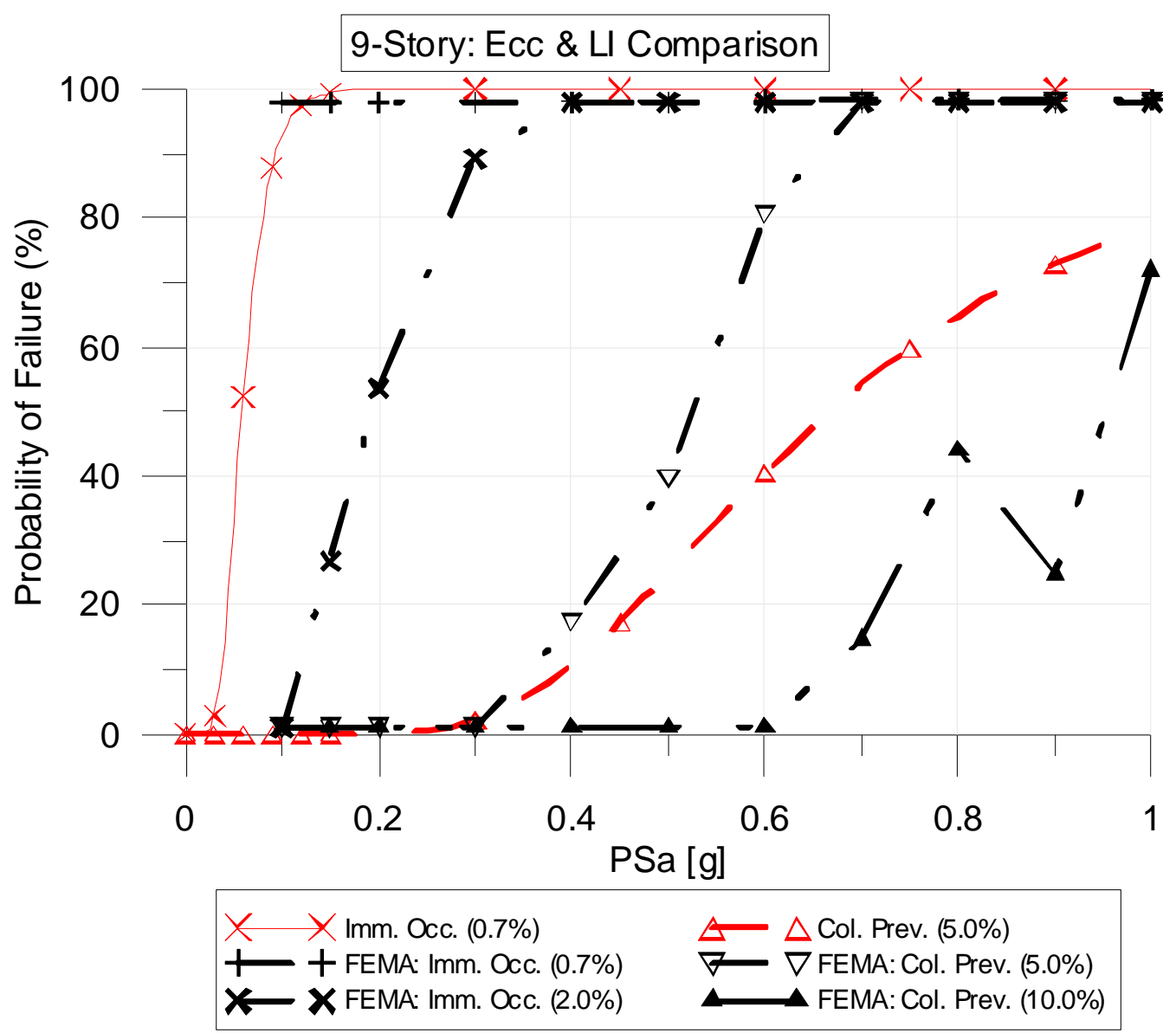

Figure 7.17: FEMA fragility curves, with code capacity and reduced capacity, compared to regression fragility curves for nine-story model

For the nine-story model, the reduction of the FEMA drift capacities again produced more comparable results, at intensities less than or equal to the MCE intensity of $0.547 \mathrm{~g}$. Fig. 7.17 above shows that at intensities greater than MCE the reduced capacity FEMA fragility curve diverges from the smooth/regression analysis and reports more conservative results. However, at reasonable intensities the reduced capacity FEMA analysis produces similar results just as with the three-story model.

For the twenty-story model the original FEMA fragility analysis was also un-conservative, but the reduced capacity FEMA fragility curve was equally shifted toward the conservative side of the smooth/regression analysis fragility curve. Looking at the collapse prevention curves in 


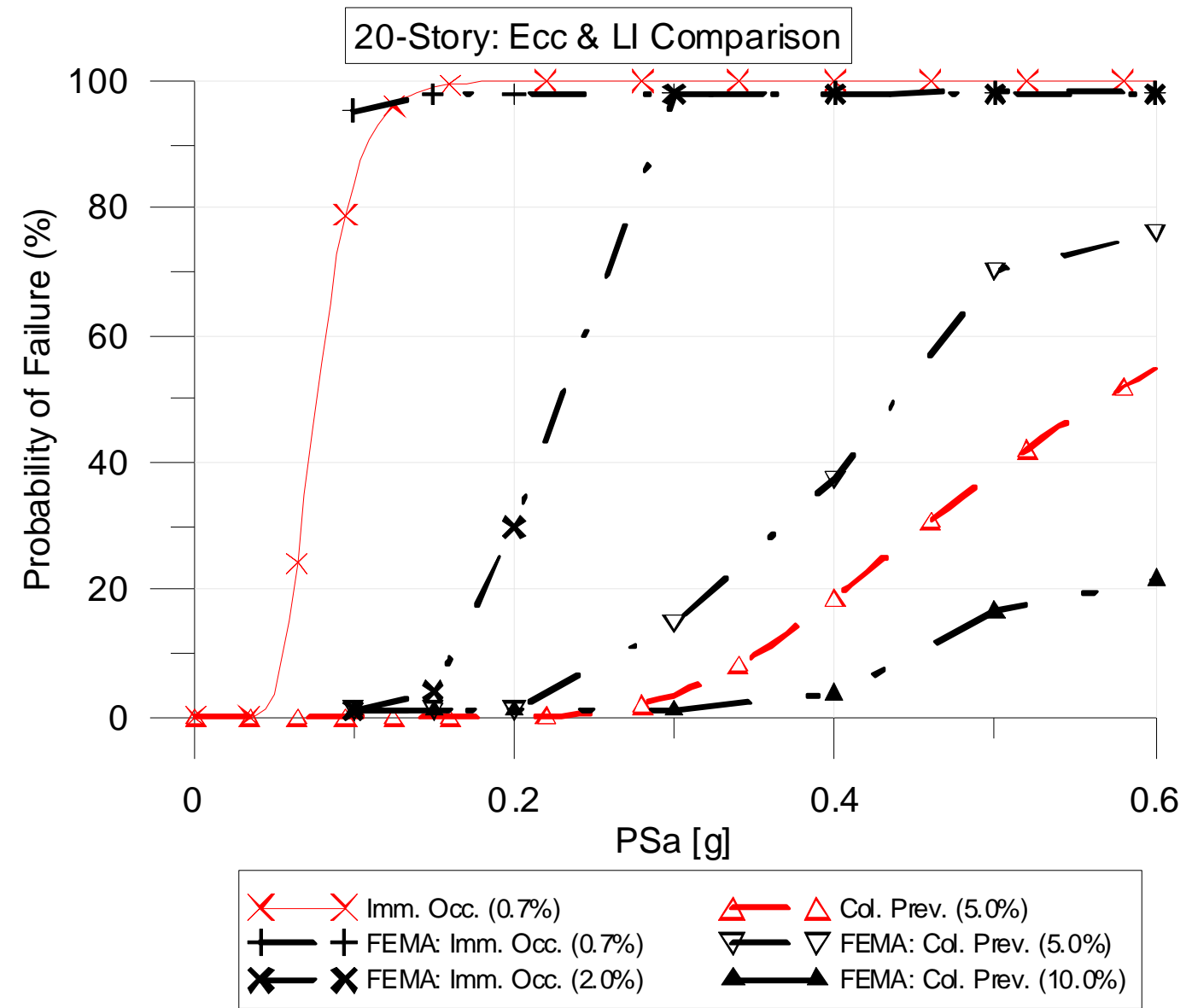

Figure 7.18: FEMA fragility curves, with code capacity and reduced capacity, compared to regression fragility curves for twenty-story model

Fig. 7.18 the smooth/regression fragility curve is equally bounded by the two sets of FEMA fragility curves, which suggests that the code applied drift capacity is too un-conservative and that the reduced capacity may be too conservative.

In conclusion, the FEMA analysis, with the larger suggested drift capacities, produces unconservative fragility curves for each of the three models. By reducing the drift capacities to more reasonable values, similar fragility curves are produced that better match those from the smooth/regression fragility analyses. 


\section{Chapter 8: Conclusions and Recommendations Future Research}

\subsection{Conclusions}

This thesis investigated the effects of uncertain reactive weight intensities and eccentricities on seismic performance of steel moment resisting frames. Three steel moment resisting frame models were used to represent a range of building heights: low-rise, mid-rise, and high-rise.

For the first model, the three-story building, the effects of considering load intensity and load eccentricity on inter-story drifts had no quantitative differences. At the DBE intensity level, all of

the loading scenarios produced drifts that were not conservatively captured for the life safety limit state, by the code applied eccentricity. While for the collapse prevention limit state, the code was able to conservatively capture the drifts for each of the loading states. For the MCE level of ground shaking, the code applied eccentricity analysis did not conservatively capture the collapse prevention drifts for any of the loading scenarios. For all of the loading scenarios the collapse margin ratios, CMR, were all about $2.0 \%$ greater than one, showing that given a MCE ground motion the building would essentially have a $50.0 \%$ probability of collapse.

For the second model, the nine-story building, the effects of considering load intensity and load eccentricity on inter-story drifts are more noticeable. Consideration of either load intensity or load eccentricity alone produced inter-story drifts that were greater than those determined without consideration of these effects. Consideration of both of these variables produced inter-story drifts that were even greater. All of the loading scenarios produced drifts that were not conservatively captured by the code at the DBE level. At the MCE level of shaking, the loading intensity and eccentricity scenario was the not conservatively captured by the code applied eccentricity

analyses. However, the CMR values for each loading scenario were all at least $20.0 \%$ greater than 
one suggesting that even though the actual drifts are not necessarily captured by the code drifts, the building has less than a $50.0 \%$ probability of failure given an MCE.

For the third model, the twenty-story building, the effects of load intensity and eccentricity on the inter-story drifts had no quantitative differences, just as with the three-story model. All of the loading scenarios produced inter-story drifts that were conservatively covered by the code eccentricity drifts at both the DBE and MCE levels. The CMR values for each loading scenario were at least $76.0 \%$ greater than one, meaning that given an MCE ground motion the building is very unlikely to experience a collapse.

Finally, the fragility curves were compared against those produced from the FEMA 350:

Performance based analysis. Significant unconservatism was observed for each of the three models at the two limit states: immediate occupancy and collapse prevention. Adjustments to the limit state capacities showed that similar fragility curves could be created with the FEMA 350 procedure.

\subsection{Recommendations for Future Research}

The torsional component of the ground motion was not considered in the nonlinear dynamic analyses that were performed. The inclusion of this component would more accurately represent an actual excitation of the building.

The models did not account for possible local failures, such as local buckling and fracture at beam-column connections. Incorporation of this into the analysis would result in a better defined model and more accurate results. 
Some ground motions were rejected from the analysis due to numerical complications of the Rosenblueth " $2 \mathrm{k}+1$ " Method. Investigation into other sampling methods could produce more reliable results without the need to increase the number of simulations.

The models used were all symmetric structures with zero stiffness eccentricity. A similar study could be done to determine the same effects on asymmetric structures. 


\section{REFERENCES}

Ang, A. H., \& Tang, W. H. (2007). Determination of Probability Distribution Models. Probability Concepts in Engineering: Emphasis on Applications in Civil \& Environmental Engineering (2nd ed., pp. 293-296). New York: Wiley.

Chang, H., Lin, C. J., Lin, K., \& Chen, J. (2009). Role of Accidental Torsion in Seismic Reliability Assessment for Steel Buildings. Steel and Composite Structures, 9(5), 457471.

Correia, A., Almeida, J., \& Pinho, R. (2008). Force-Based Versus Displacement-Based Formulations In The Cyclic Nonlinear Analysis of RC Frames. The 14th World Conference on Earthquake Engineering Innovation, Practice, Safety: October 12-17, 2008, Beijing, China (p. n/a). Japan: IAEE].

De-la-Colina, J., Benitez, B., \& Ruiz, S. E. (2011). Accidental Eccentricity of Story Shear for Low-Rise Office Buildings. Journal of Structural Engineering, 137(4), 513-520.

Fahjan, Y. M., Tuzun, C., \& Kubin, J. (2006). An Alternative Procedure for Accidental Eccentricity in Dynamic Model Analyses of Buildings. First European Conference on Earthquake Engineering and Seismology 3-8 September, 2006, Geneva, Switzerland (p. Paper No. 1166). Geneva: s.n.

FEMA 356: Prestandard and Commentary for the Seismic Rehabilitation of Buildings (pp. 1-35). (2000). Rehabilitation Requirements. Reston, Va.: American Society of Civil Engineers.

FEMA P695: Quantification of Building Seismic Performance Factors. (2009). Washington, D.C.: U.S. Dept. of Homeland Security, FEMA.

FEMA 350: Recommended Seismic Design Criteria for New Steel Moment-Frame Buildings. (2000). Washington, D.C.: Federal Emergency Management Agency.

Gupta, A., \& Krawinkler, H. (1999).Seismic Demands For Performance Evaluation of Steel Moment Resisting Frame Structures (SAC task 5.4.3). Stanford, Calif.: The John A. Blume Earthquake Engineering Center.

Minimum Design Loads for Buildings and Other Structures (pp. 88-89). (2010). Seismic Design Requirements for Building Structures. Reston, Va.: American Society of Civil Engineers.

Nowak, A. S., \& Collins, K. R. (2000). Simulation Techniques. Reliability of structures (pp. 6988). Boston: McGraw-Hill.

Scott, Michael H. (2011). Force-Based Element Integration Options in OpenSees: Numerical Integration Options for the Force-Based Beam-Column Element in OpenSees. Retrieved March 3, 2011, from http://opensees.berkeley.edu/wiki/images/a/ab/IntegrationTypes.pdf.

Stathopoulos, K., \& Anagnostopoulos, S. (2005). Inelastic Torsion of Multistorey Buildings Under Earthquake Excitations. Earthquake Engineering and Structural Dynamics, 34(12), 1449-1465. Retrieved September 20, 2011, from http://onlinelibrary.wiley.com/doi/10.1002/eqe.486/abstract 
Stathopoulos, K., \& Anagnostopoulos, S. (2000). Inelastic Earthquake Response of Buildings Subjected to Torsion. 12 WCEE 2000: 12th World Conference on Earthquake Engineering.(p. n/a). New Zealand: New Zealand National Society for Earthquake Engineering.

Tso, W., \& Dempsey, K. (1980). Seismic Torsional Provisions for Dynamic Eccentricity. Earthquake Engineering and Structural Dynamics, 8, 275-289. 

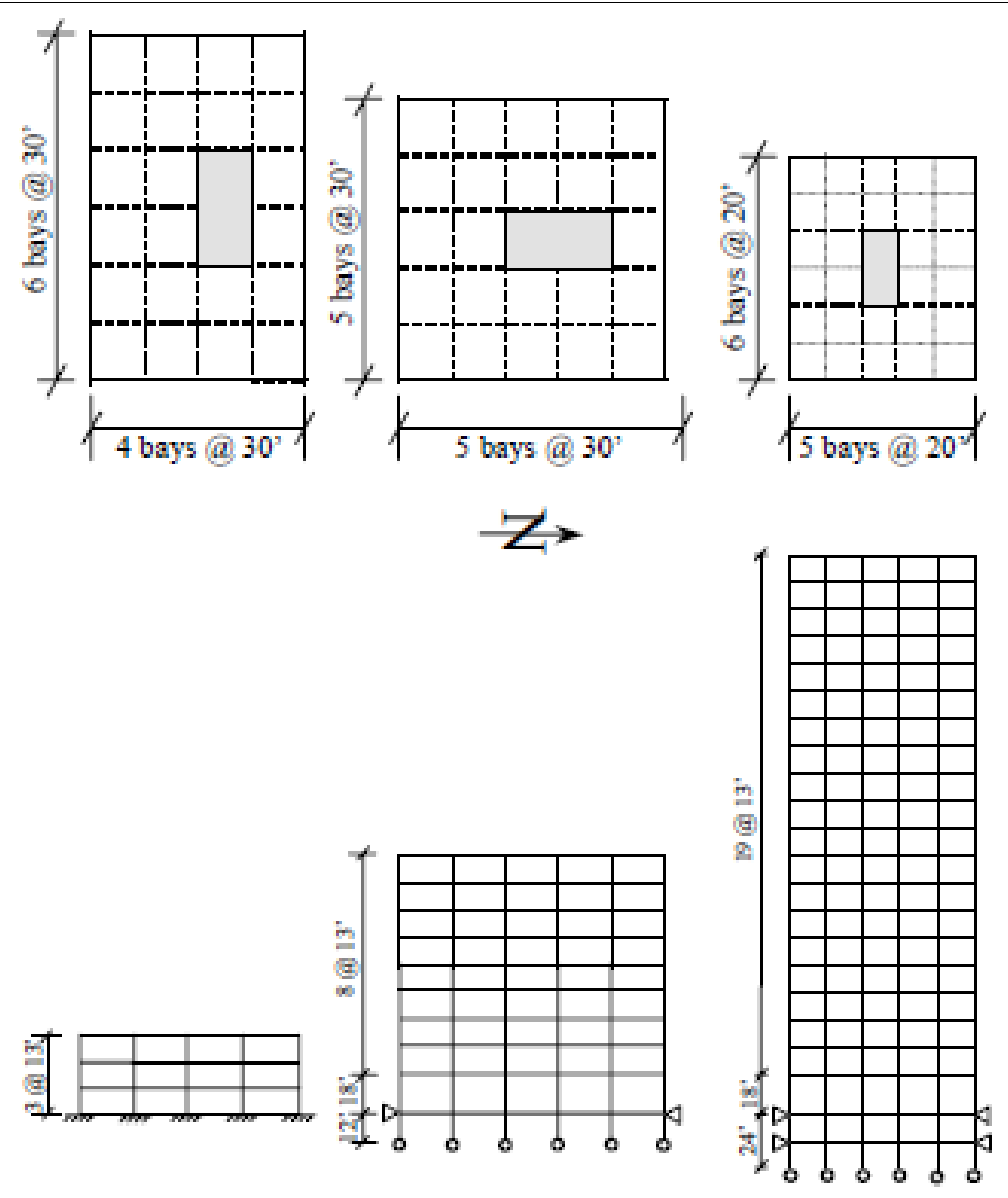

Figure B.1 Floor Plans and Elevations for Model Buildings
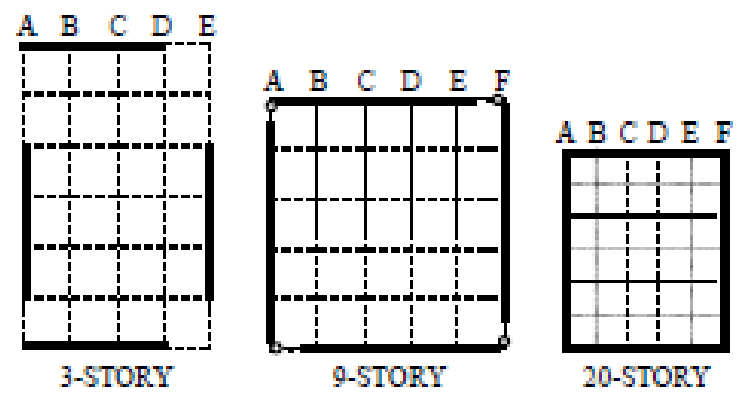

Figure B.2 Floor Plans Showing Layout of Moment Resisting Frames for LA Model Buildings

Figure A.1: Building layouts from Gupta et al. (1999) 
Table A.1: Intermediate floors for 3-,9-, and 20-story load parameters

\begin{tabular}{llll}
\hline & & Mean, $\mu$ & Standard Deviation, $\sigma$ \\
\hline \multirow{2}{*}{ Dead Load Parameters } & Position_X_Dead & 0.5 & 0.025 \\
& Position_Y_Dead & 0.5 & 0.025 \\
& Load_Intensity_Dead (psf) & 86 & 8.6 \\
\hline \multirow{2}{*}{ Live Load Parameters } & Position_X_Live & 0.5 & 0.037 \\
& Position_Y_Live & 0.5 & 0.037 \\
& Load_Intensity_Live (psf) & 10 & 2.72 \\
\hline \multirow{2}{*}{ Effective Load Parameters } & Position_X_Effective & 0.5 & 0.0228 \\
& Position_Y_Effective & 0.5 & 0.0228 \\
& Load_Intensity_Effective (psf) & 95.99 & 9.0193 \\
\hline
\end{tabular}

Table A.2: 3-Story roof with penthouse load parameters

\begin{tabular}{llll}
\hline & & Mean, $\mu$ & Standard Deviation, $\sigma$ \\
\hline Dead Load Parameters & Position_X_Dead & 0.5 & 0.025 \\
& Position_Y_Dead & 0.5 & 0.025 \\
& Load_Intensity_Dead (psf) & 92.7 & 8.6 \\
\hline \multirow{2}{*}{ Live Load Parameters } & Position_X_Live & 0.5 & 0.037 \\
& Position_Y_Live & 0.5 & 0.037 \\
& Load_Intensity_Live (psf) & 10 & 2.72 \\
\hline \multirow{2}{*}{ Effective Load Parameters } & Position_X_Effective & 0.5 & 0.0229 \\
& Position_Y_Effective & 0.5 & 0.0229 \\
& Load_Intensity_Effective (psf) & 102.70 & 9.6611 \\
\hline
\end{tabular}

Table A.3: 9-Story roof with penthouse load parameters

\begin{tabular}{llll}
\hline & & Mean, $\mu$ & Standard Deviation, $\sigma$ \\
\hline Dead Load Parameters & Position_X_Dead & 0.5 & 0.025 \\
& Position_Y_Dead & 0.5 & 0.025 \\
& Load_Intensity_Dead (psf) & 92.3 & 8.6 \\
\hline Live Load Parameters & Position_X_Live & 0.5 & 0.037 \\
& Position_Y_Live & 0.5 & 0.037 \\
& Load_Intensity_Live (psf) & 10 & 2.72 \\
\hline \multirow{2}{*}{ Effective Load Parameters } & Position_X_Effective & 0.5 & 0.0229 \\
& Position_Y_Effective & 0.5 & 0.0229 \\
& Load_Intensity_Effective (psf) & 102.30 & 9.6226 \\
\hline
\end{tabular}


Table A.4: 20-Story roof with penthouse load parameters

\begin{tabular}{llll}
\hline & & Mean, $\mu$ & Standard Deviation, $\sigma$ \\
\hline Dead Load Parameters & Position_X_Dead & 0.5 & 0.025 \\
& Position_Y_Dead & 0.5 & 0.025 \\
& Load_Intensity_Dead (psf) & 90.7 & 8.6 \\
\hline Live Load Parameters & Position_X_Live & 0.5 & 0.037 \\
& Position_Y_Live & 0.5 & 0.037 \\
& Load_Intensity_Live (psf) & 10 & 2.72 \\
\hline Effective Load Parameters & Position_X_Effective & 0.5 & 0.0228 \\
& Position_Y_Effective & 0.5 & 0.0228 \\
& Load_Intensity_Effective (psf) & 100.69 & 9.4663 \\
\hline
\end{tabular}




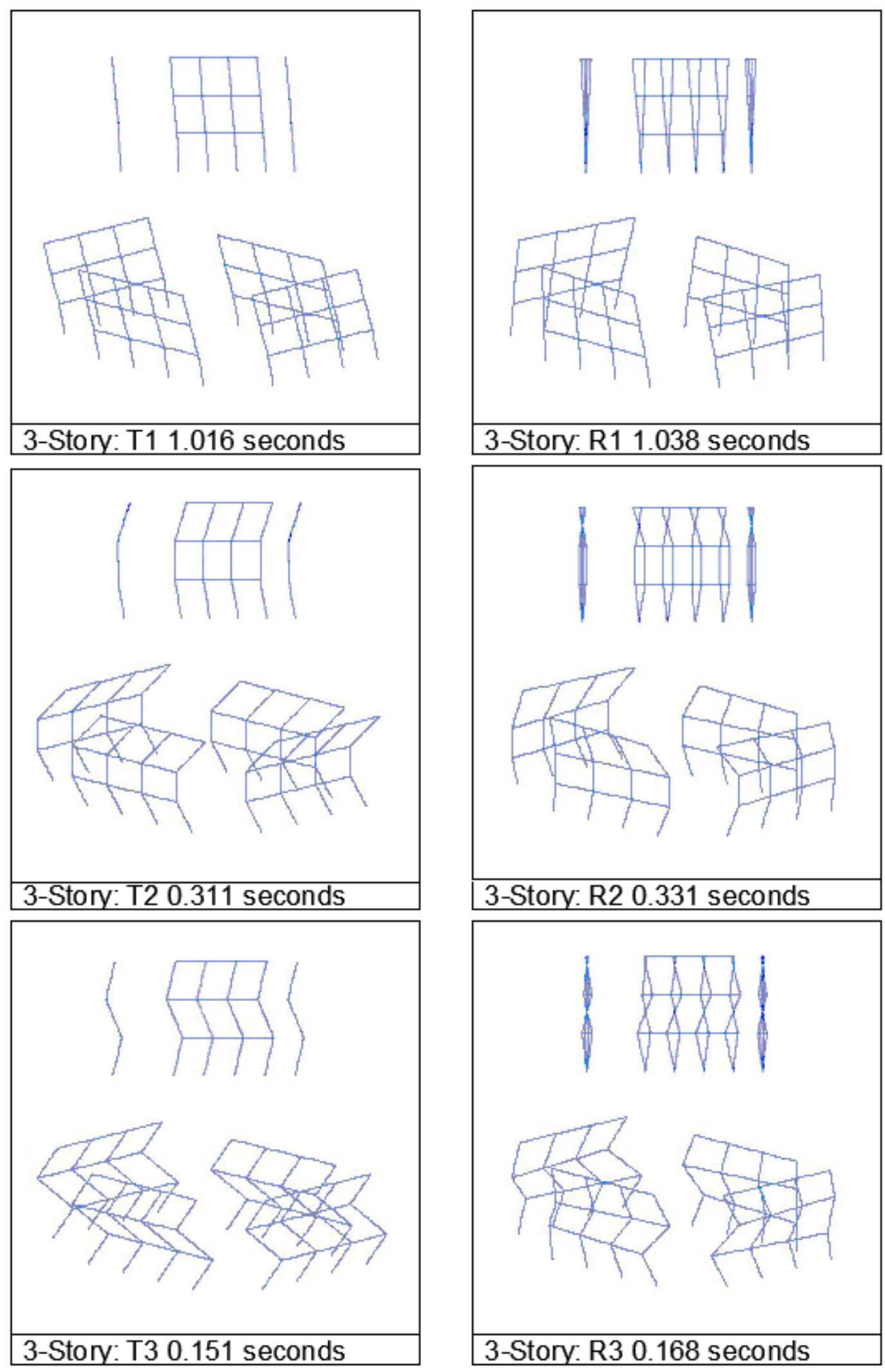

Figure A.2: Three-story mode shapes 

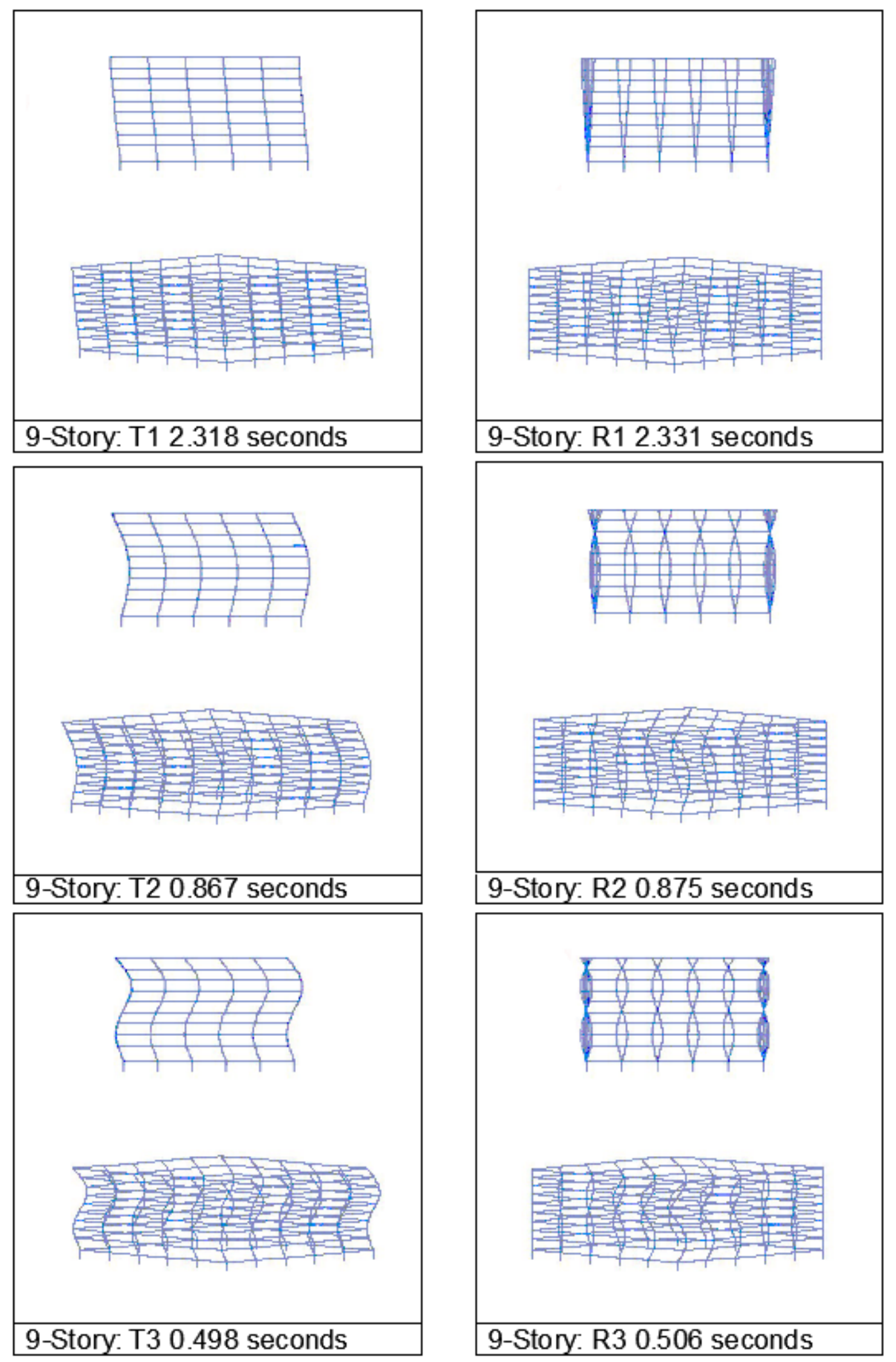

Figure A.3: Nine-story mode shapes 

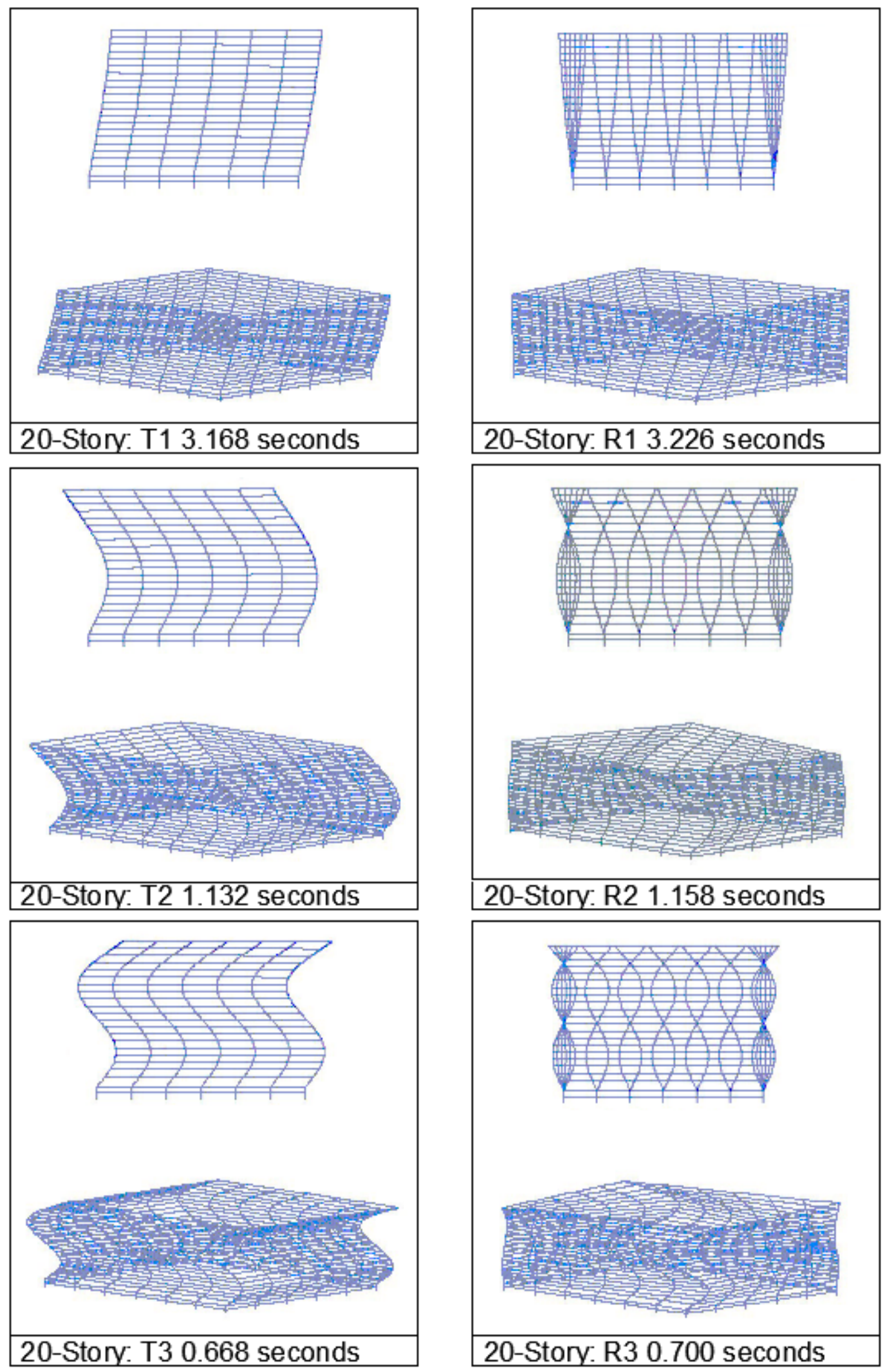

Figure A.4: Twenty-story mode shapes 


\section{Appendix B: Disqualification of Particular Ground Motions}

Analysis of the drifts determined from the Rosenblueth " $2 \mathrm{k}+1$ " Point Estimate Sampling Method showed that at larger ground motion intensities some of the individual ground motions reported very large drifts. Examination of these particular instances revealed that the individual simulations reported reasonable and expected drift values. The combination of the individual drifts is what resulted in the very large reported drifts. The individual drifts are combined in accordance with Eq. 5.7 from Section 5.2

$$
\overline{\mathrm{Y}}=y_{o} \prod_{1=1}^{K}\left(\frac{\bar{y}_{i}}{y_{o}}\right)
$$

Each of the individual simulation results, $\mathrm{y}_{\mathrm{i}}$, is normalized by the mean simulation drift value, $\mathrm{y}_{0}$, and acts as a small amplification factor of the mean simulation drift. These individual

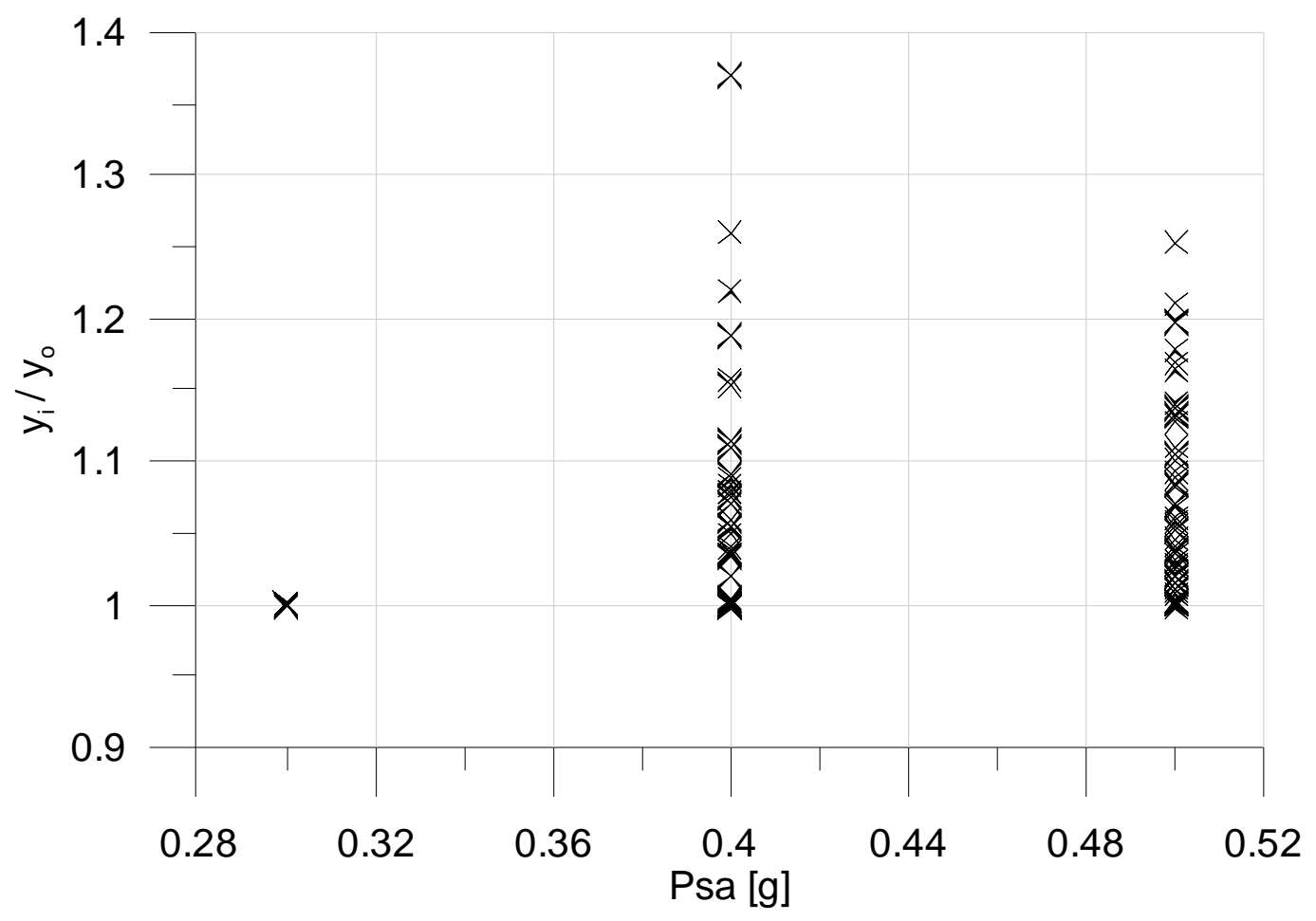

Figure B.1: Normalized Rosenblueth " $2 \mathrm{k}+1$ ” results 
amplification values are multiplied together to produce an effective amplification factor. When an individual simulation drift is greater than the mean scenario drift value the result is an amplification that is greater than one. When the majority of the individual factors are greater than one the result is an effective amplification that is much greater than one. This amplification problem becomes more prolific as the number of simulations increases because the number of factors being multiplied together increases. Therefore, more ground motions result in unrealistic drifts for the 20-story model compared to the 3-story model.

Fig. B.1 above shows the individual normalized amplification factors for one particular ground motion at several different intensities. They have all been normalized by the mean simulation drift. Therefore, a value greater than one will amplify the end result, whereas a value less than one will decrease the end result. At an intensity of $0.3 \mathrm{~g}$ all of the points are very close to a value of one, resulting in minimal amplification. However, when the intensity is increased to $0.4 \mathrm{~g}$ and $0.5 \mathrm{~g}$ the majority of the individual points are greater than one and the end result is an amplification of ten or more of the mean simulation drift. Though many of the individual amplification factors are reasonable when they are multiplied together to the $\mathrm{n}^{\text {th }}$ degree the result is an unrealistic amplification.

This problem is inherent to the numerical combination of the Rosenblueth " $2 \mathrm{k}+1$ " Method and cannot be avoided. Therefore, the ground motions for each model that reported these unrealistic values were disregarded for the entire analysis. This only resulted in one ground motion being removed from the 3-Story analysis, two for the 9-Story analysis, and five for the 20-Story analysis. There were still enough ground motions for each model to develop unbiased distributions. 


\section{APPENDIX C: USGS Detailed Report}

$9 / 5 / 12$

Design Maps Detailed Report

\section{ZUSGS Design Maps Detailed Report}

ASCE 7-10 Standard $\left(34.05008^{\circ} \mathrm{N}, 118.24225^{\circ} \mathrm{W}\right)$

\section{Section 11.4.1 - Mapped Acceleration Parameters}

Note: Ground motion values provided below are for the direction of maximum horizontal spectral response acceleration. They have been converted from corresponding geometric mean ground motions computed by the USGS by applying factors of 1.1 (to obtain $\mathrm{S}_{\mathrm{s}}$ ) and 1.3 (to obtain $\mathrm{S}_{1}$ ). Maps in the 2010 ASCE-7 Standard are provided for Site Class B. Adjustments for other Site Classes are made, as needed, in Section 11.4.3.

From Eigure 22-1
From Fiqure 22-2 ${ }^{\text {[2] }}$
$\mathrm{S}_{1}=0.846 \mathrm{~g}$

\section{Section 11.4.2 - Site Class}

The authority having jurisdiction (not the USGS), site-specific geotechnical data, and/or the default has classified the site as Site Class D, based on the site soil properties in accordance with Chapter 20.

Table 20.3-1 Site Classification

\begin{tabular}{lccc} 
Site Class & $\bar{v}_{\mathrm{s}}$ & $\overline{\boldsymbol{N}}$ or $\overline{\boldsymbol{N}}_{\mathrm{ch}}$ & $\overline{\boldsymbol{s}}_{\mathrm{u}}$ \\
\hline A. Hard Rock & $>5,000 \mathrm{ft} / \mathrm{s}$ & $\mathrm{N} / \mathrm{A}$ & $\mathrm{N} / \mathrm{A}$ \\
\hline B. Rock & 2,500 to $5,000 \mathrm{ft} / \mathrm{s}$ & $\mathrm{N} / \mathrm{A}$ & $\mathrm{N} / \mathrm{A}$ \\
\hline C. Very dense soil and soft rock & 1,200 to $2,500 \mathrm{ft} / \mathrm{s}$ & $>50$ & $>2,000 \mathrm{psf}$ \\
\hline D. Stiff Soil & 600 to $1,200 \mathrm{ft} / \mathrm{s}$ & 15 to 50 & 1,000 to $2,000 \mathrm{psf}$ \\
\hline E. Soft clay soil & $<600 \mathrm{ft} / \mathrm{s}$ & $<15$ & $<1,000 \mathrm{psf}$ \\
& $\begin{array}{l}\text { Any profile with more than } 10 \mathrm{ft} \text { of soil having the } \\
\text { characteristics: } \\
\end{array}$ & $\begin{array}{l}\text { Plasticity index } P I>20, \\
\end{array}$ & Moisture content $w \geq 40 \%$, and \\
& - Undrained shear strength $\bar{s}_{\mathrm{u}}<500 \mathrm{psf}$ & \\
\hline
\end{tabular}

F. Soils requiring site response

See Section 20.3.1 analysis in accordance with Section 21.1

For SI: $1 \mathrm{ft} / \mathrm{s}=0.3048 \mathrm{~m} / \mathrm{s} 1 \mathrm{lb} / \mathrm{ft}^{2}=0.0479 \mathrm{kN} / \mathrm{m}^{2}$ 
Section 11.4.3 - Site Coefficients and Risk-Targeted Maximum Considered Earthquake $\left(\mathrm{MCE}_{\mathrm{R}}\right)$ Spectral Response Acceleration Parameters

Table 11.4-1: Site Coefficient $\mathrm{F}_{\mathrm{a}}$

\begin{tabular}{cccccc}
\hline \multirow{2}{*}{$\begin{array}{c}\text { Site } \\
\text { Class }\end{array}$} & \multicolumn{5}{c}{$\begin{array}{c}\text { Mapped } \mathrm{MCE}_{\mathrm{R}} \text { Spectral Response Acceleration Parameter at Short } \\
\text { Period }\end{array}$} \\
\cline { 2 - 6 } & $\mathrm{S}_{\mathrm{S}} \leq 0.25$ & $\mathrm{~S}_{\mathrm{S}}=0.5$ & $\mathrm{~S}_{\mathrm{S}}=0.75$ & $\mathrm{~S}_{\mathrm{S}}=1$ & $\mathrm{~S}_{\mathrm{S}} \geq 1.25$ \\
\hline $\mathrm{A}$ & 0.8 & 0.8 & 0.8 & 0.8 & 0.8 \\
$\mathrm{~B}$ & 1.0 & 1.0 & 1.0 & 1.0 & 1.0 \\
$\mathrm{C}$ & 1.2 & 1.2 & 1.1 & 1.0 & 1.0 \\
$\mathrm{D}$ & 1.6 & 1.4 & 1.2 & 1.1 & 1.0 \\
$\mathrm{E}$ & 2.5 & 1.7 & 1.2 & 0.9 & 0.9 \\
$\mathrm{~F}$ & & See Section 11.4 .7 of ASCE 7 & \\
\hline
\end{tabular}

Note: Use straight-line interpolation for intermediate values of $\mathrm{S}_{\mathrm{S}}$

For Site Class $=D$ and $S_{\mathrm{s}}=2.415 \mathrm{~g}, \mathrm{~F}_{\mathrm{a}}=\mathbf{1 . 0 0 0}$

Table 11.4-2: Site Coefficient $F_{v}$

\begin{tabular}{cccccc}
\hline \hline \multirow{2}{*}{$\begin{array}{c}\text { Site } \\
\text { Class }\end{array}$} & \multicolumn{5}{c}{ Mapped $\mathrm{MCE}_{\mathrm{R}}$ Spectral Response Acceleration Parameter at 1-s } \\
& \multicolumn{5}{c}{ Period } \\
\cline { 2 - 6 } & $\mathrm{S}_{1} \leq 0.1$ & $\mathrm{~S}_{1}=0.2$ & $\mathrm{~S}_{1}=0.3$ & $\mathrm{~S}_{1}=0.4$ & $\mathrm{~S}_{1} \geq 0.5$ \\
\hline $\mathrm{A}$ & 0.8 & 0.8 & 0.8 & 0.8 & 0.8 \\
B & 1.0 & 1.0 & 1.0 & 1.0 & 1.0 \\
C & 1.7 & 1.6 & 1.5 & 1.4 & 1.3 \\
D & 2.4 & 2.0 & 1.8 & 1.6 & 1.5 \\
E & 3.5 & 3.2 & 2.8 & 2.4 & 2.4 \\
F & & See Section 11.4.7 of ASCE 7 & \\
\hline
\end{tabular}

Note: Use straight-line interpolation for intermediate values of $\mathrm{S}_{1}$

For Site Class $=D$ and $S_{1}=0.846 \mathrm{~g}, F_{v}=1.500$ 
Equation (11.4-1):

Equation (11.4-2):

$$
\mathrm{S}_{\mathrm{MS}}=\mathrm{F}_{\mathrm{a}} \mathrm{S}_{\mathrm{S}}=1.000 \times 2.415=2.415 \mathrm{~g}
$$

$$
\mathrm{S}_{\mathrm{M} 1}=\mathrm{F}_{\mathrm{v}} \mathrm{S}_{1}=1.500 \times 0.846=1.269 \mathrm{~g}
$$

Section 11.4.4 - Design Spectral Acceleration Parameters

Equation (11.4-3):

Equation (11.4-4):

$$
\mathrm{S}_{\mathrm{DS}}=2 / 3 \mathrm{~S}_{\mathrm{MS}}=2 / 3 \times 2.415=1.610 \mathrm{~g}
$$

$\mathrm{S}_{\mathrm{D} 1}=2 / 3 \mathrm{~S}_{\mathrm{M} 1}=2 / 3 \times 1.269=0.846 \mathrm{~g}$

Section 11.4.5 - Design Response Spectrum

From Fiqure 22-12 ${ }^{[3]}$

$\mathrm{T}_{\mathrm{L}}=8$ seconds

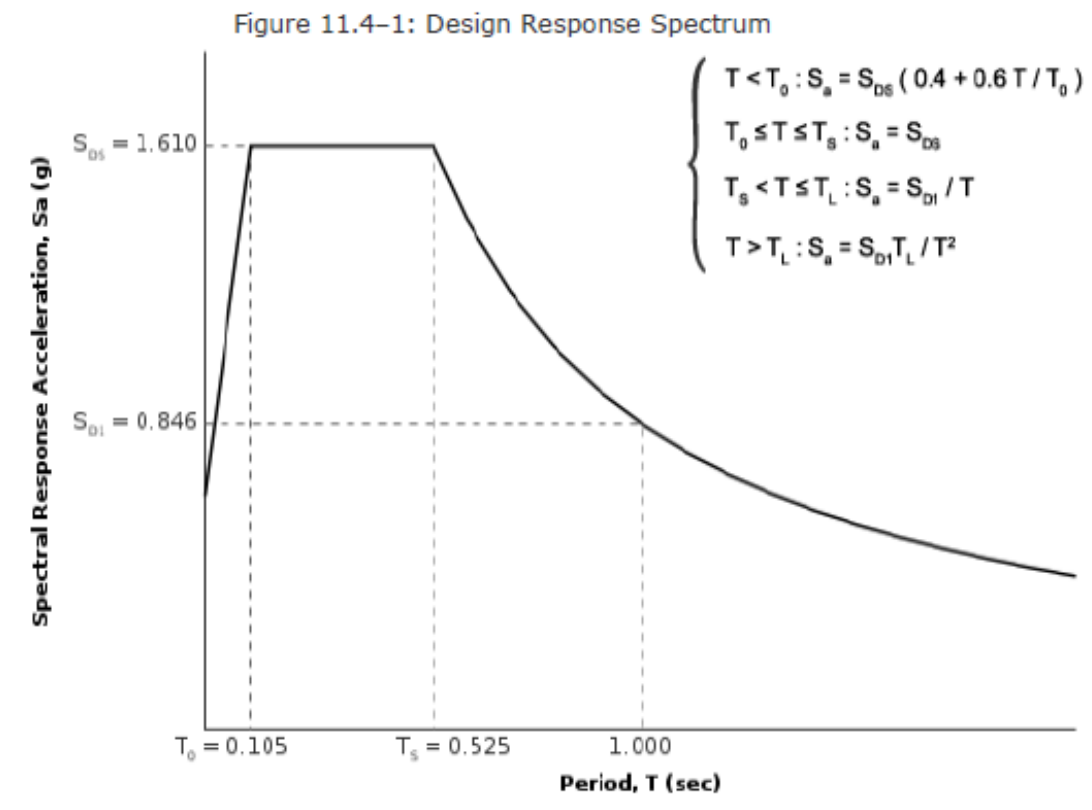


Section 11.4.6 - Risk-Targeted Maximum Considered Earthquake $\left(M_{C} E_{R}\right)$ Response Spectrum The $\mathrm{MCE}_{\mathrm{R}}$ Response Spectrum is determined by multiplying the design response spectrum above by 1.5 .

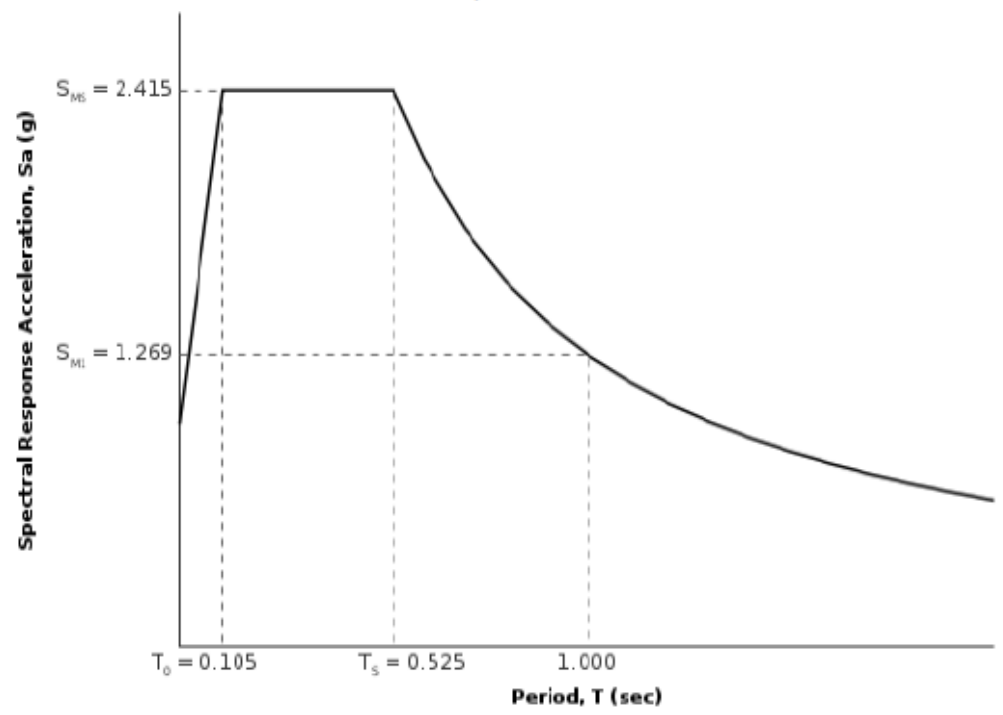


Section 11.8.3 - Additional Geotechnical Investigation Report Requirements for Seismic Design Categories D through $\mathrm{F}$

\begin{tabular}{|c|c|c|c|c|c|}
\hline \multicolumn{5}{|c|}{ From Fiqure 22-7 ${ }^{[4]}$} & $P G A=0$. \\
\hline \multicolumn{3}{|c|}{ Equation (11.8-1): } & \multicolumn{3}{|c|}{$\mathrm{PGA}_{\mathrm{M}}=\mathrm{F}_{\mathrm{PGA}} \mathrm{PGA}=1.000 \times 0.912=0.9$} \\
\hline \multicolumn{6}{|c|}{ Table 11.8-1: Site Coefficient $F_{P G a}$} \\
\hline \multirow[t]{2}{*}{ Site Class } & \multicolumn{5}{|c|}{ Mapped MCE Geometric Mean Peak Ground Acceleration, PGA } \\
\hline & $P G A \leq 0.1$ & $\mathrm{PGA}=0.2$ & $\mathrm{PGA}=0.3$ & $\mathrm{PGA}=0.4$ & $P G A \geq 0.5$ \\
\hline A & 0.8 & 0.8 & 0.8 & 0.8 & 0.8 \\
\hline B & 1.0 & 1.0 & 1.0 & 1.0 & 1.0 \\
\hline $\mathrm{C}$ & 1.2 & 1.2 & 1.1 & 1.0 & 1.0 \\
\hline $\mathrm{D}$ & 1.6 & 1.4 & 1.2 & 1.1 & 1.0 \\
\hline $\mathrm{E}$ & 2.5 & 1.7 & 1.2 & 0.9 & 0.9 \\
\hline $\mathrm{F}$ & \multicolumn{5}{|c|}{ See Section 11.4 .7 of ASCE 7} \\
\hline
\end{tabular}

Note: Use straight-line interpolation for intermediate values of PGA

For Site Class $=\mathrm{D}$ and $\mathrm{PGA}=\mathbf{0 . 9 1 2} \mathrm{g}, \mathrm{F}_{\mathrm{PGA}}=\mathbf{1 . 0 0 0}$

Section 21.2.1.1 - Method 1 (from Chapter 21 - Site-Specific Ground Motion Procedures for Seismic Design)

From Fiqure 22-17 ${ }^{\text {[5] }}$

$C_{\text {RS }}=0.942$

From Figure 22-18 ${ }^{\text {[6] }}$

$C_{R 1}=0.958$ 
Section 11.6 - Seismic Design Category

Table 11.6-1 Seismic Design Category Based on Short Period Response Acceleration Parameter

\begin{tabular}{|c|c|c|c|}
\hline \multirow{2}{*}{ VALUE OF $S_{\mathrm{DS}}$} & \multicolumn{3}{|c|}{ RISK CATEGORY } \\
\cline { 2 - 4 } & I Or II & III & IV \\
\hline $\mathrm{S}_{\mathrm{DS}}<0.167 \mathrm{~g}$ & $\mathrm{~A}$ & A \\
\hline $0.167 \mathrm{~g} \leq \mathrm{S}_{\mathrm{DS}}<0.33 \mathrm{~g}$ & B & B & C \\
\hline $0.33 \mathrm{~g} \leq \mathrm{S}_{\mathrm{DS}}<0.50 \mathrm{~g}$ & $\mathrm{C}$ & $\mathrm{C}$ & $\mathrm{D}$ \\
\hline $0.50 \mathrm{~g} \leq \mathrm{S}_{\mathrm{DS}}$ & $\mathrm{D}$ & $\mathrm{D}$ & $\mathrm{D}$ \\
\hline
\end{tabular}

For Risk Category $=\mathrm{I}$ and $\mathrm{S}_{\mathrm{DS}}=\mathbf{1 . 6 1 0} \mathrm{g}$, Seismic Design Category = D

Table 11.6-2 Seismic Design Category Based on 1-S Period Response Acceleration Parameter

\begin{tabular}{|c|c|c|c|}
\hline \multirow{2}{*}{ VALUE OF $\mathrm{S}_{\mathrm{D} 1}$} & \multicolumn{3}{|c|}{ RISK CATEGORY } \\
\cline { 2 - 4 } & I Or II & III & IV \\
\hline $\mathrm{S}_{\mathrm{D} 1}<0.067 \mathrm{~g}$ & $\mathrm{~A}$ & $\mathrm{~A}$ & $\mathrm{~A}$ \\
\hline $0.067 \mathrm{~g} \leq \mathrm{S}_{\mathrm{D} 1}<0.133 \mathrm{~g}$ & $\mathrm{~B}$ & $\mathrm{~B}$ & $\mathrm{C}$ \\
\hline $0.133 \mathrm{~g} \leq \mathrm{S}_{\mathrm{D} 1}<0.20 \mathrm{~g}$ & $\mathrm{C}$ & $\mathrm{C}$ & $\mathrm{D}$ \\
\hline $0.20 \mathrm{~g} \leq \mathrm{S}_{\mathrm{D} 1}$ & $\mathrm{D}$ & $\mathrm{D}$ & $\mathrm{D}$ \\
\hline
\end{tabular}

For Risk Category $=\mathrm{I}$ and $\mathrm{S}_{\mathrm{D} 1}=\mathbf{0 . 8 4 6} \mathrm{g}$, Seismic Design Category = D

Note: When $S_{1}$ is greater than or equal to $0.75 \mathrm{~g}$, the Seismic Design Category is $\mathbf{E}$ for buildings in Risk Categories I, II, and III, and F for those in Risk Category IV, irrespective of the above.

Seismic Design Category $\equiv$ "the more severe design category in accordance with Table $11.6-1$ or $11.6-2^{\prime \prime}=\mathrm{E}$

Note: See Section $\mathbf{1 1 . 6}$ for alternative approaches to calculating Seismic Design Category.

References

1. Figure 22-1: http://earthquake.usgs.gov/hazards/designmaps/downloads/pdfs/2010_ASCE7_Figure_22-1.pdf

2. Figure 22-2: http://earthquake.usgs.gov/hazards/designmaps/downloads/pdfs/2010_ASCE7_Figure_22-2.pdf

3. Figure 22-12: http://earthquake.usgs.gov/hazards/designmaps/downloads/pdfs/2010_ASCE7_Figure_22-12.pdf

4. Figure 22-7: http://earthquake.usgs.gov/hazards/designmaps/downloads/pdfs/2010_ASCE7_Figure_22-7.pdf

5. Figure 22-17: http://earthquake.usgs.gov/hazards/designmaps/downloads/pdfs/2010_ASCE7_Figure_22-17.pdf

6. Figure 22-18: http://earthquake.usgs.gov/hazards/designmaps/downloads/pdfs/2010_ASCE7_Figure_22-18.pdf 


\section{APPENDIX D: Drift Results}

The tables in Appendix D report the maximum inter-story drifts for all of the loading scenarios. The maximum drifts from the individual simulations have already been combined with the Rosenbleuth " $2 \mathrm{k}+1$ " Method and separated into the various loading scenarios: load eccentricity and intensity combined, load eccentricity alone, load intensity alone, and neither load eccentricity, nor load intensity (mean scenario). Each of the three different models has a table for each of the four loading scenarios mentioned above. In addition to these results, the results of the code applied eccentricity drifts are tabulated for the DBE and MCE intensities.

As mentioned in Appendix B, certain ground motions were disqualified for each model. The disqualified ground motions are represented with dash marks in lieu of a story drift percent. 
Table D.1: 3-Story drifts for load eccentricity and intensity

\begin{tabular}{|c|c|c|c|c|c|c|c|c|c|c|c|}
\hline $\begin{array}{l}\text { Ground } \\
\text { Motion }\end{array}$ & $\begin{array}{l}\text { PSa }(g) \\
0.01\end{array}$ & 0.1 & 0.2 & 0.3 & 0.4 & 0.5 & 0.6 & 0.7 & 0.8 & 0.9 & 1.0 \\
\hline 1 & $0.046 \%$ & $0.462 \%$ & $0.849 \%$ & $1.083 \%$ & $1.407 \%$ & $1.616 \%$ & $1.847 \%$ & $1.996 \%$ & $2.133 \%$ & $2.430 \%$ & $2.665 \%$ \\
\hline 2 & $0.039 \%$ & $0.393 \%$ & $0.790 \%$ & $1.115 \%$ & $1.683 \%$ & $1.951 \%$ & $2.175 \%$ & $2.359 \%$ & $2.557 \%$ & $2.757 \%$ & $2.955 \%$ \\
\hline 3 & $0.038 \%$ & $0.384 \%$ & $0.769 \%$ & $1.047 \%$ & $1.371 \%$ & $1.774 \%$ & $2.101 \%$ & $2.331 \%$ & $2.496 \%$ & $2.642 \%$ & $2.790 \%$ \\
\hline 4 & $0.052 \%$ & $0.523 \%$ & $1.001 \%$ & $1.117 \%$ & $1.851 \%$ & $1.995 \%$ & $2.431 \%$ & $2.895 \%$ & $3.335 \%$ & $3.761 \%$ & $4.107 \%$ \\
\hline 5 & $0.040 \%$ & $0.399 \%$ & $0.794 \%$ & $1.129 \%$ & $1.461 \%$ & $1.812 \%$ & $2.121 \%$ & $2.529 \%$ & $2.927 \%$ & $3.225 \%$ & $3.521 \%$ \\
\hline 6 & $0.053 \%$ & $0.511 \%$ & $0.991 \%$ & $1.630 \%$ & $2.040 \%$ & $2.761 \%$ & $3.340 \%$ & $4.006 \%$ & $4.310 \%$ & $4.469 \%$ & $4.621 \%$ \\
\hline 7 & $0.050 \%$ & $0.503 \%$ & $0.984 \%$ & $2.181 \%$ & $2.045 \%$ & $2.422 \%$ & $2.890 \%$ & $3.271 \%$ & $3.597 \%$ & $4.130 \%$ & $4.466 \%$ \\
\hline 8 & $0.039 \%$ & $0.390 \%$ & $0.780 \%$ & $1.330 \%$ & $2.149 \%$ & $2.700 \%$ & $3.115 \%$ & $3.224 \%$ & $3.762 \%$ & $3.873 \%$ & $4.492 \%$ \\
\hline 9 & $0.042 \%$ & $0.424 \%$ & $0.826 \%$ & $1.134 \%$ & $1.451 \%$ & $1.907 \%$ & $2.409 \%$ & $2.867 \%$ & $3.327 \%$ & $3.767 \%$ & $4.206 \%$ \\
\hline $10^{*}$ & ---- & ---- & ---- & ---- & ---- & ---- & ---- & ---- & ---- & ---- & ---- \\
\hline 11 & $0.039 \%$ & $0.394 \%$ & $0.790 \%$ & $1.118 \%$ & $1.363 \%$ & $1.518 \%$ & $1.641 \%$ & $1.906 \%$ & $2.133 \%$ & $2.584 \%$ & $3.014 \%$ \\
\hline 12 & $0.051 \%$ & $0.514 \%$ & $1.191 \%$ & $1.765 \%$ & $1.998 \%$ & $2.539 \%$ & $3.529 \%$ & $3.961 \%$ & $4.628 \%$ & $5.317 \%$ & $5.675 \%$ \\
\hline 13 & $0.052 \%$ & $0.520 \%$ & $1.107 \%$ & $1.519 \%$ & $1.743 \%$ & $2.327 \%$ & $2.497 \%$ & $2.804 \%$ & $3.048 \%$ & $3.472 \%$ & $3.847 \%$ \\
\hline 14 & $0.045 \%$ & $0.453 \%$ & $0.886 \%$ & $1.315 \%$ & $1.895 \%$ & $2.475 \%$ & $2.920 \%$ & $3.347 \%$ & $3.704 \%$ & $4.081 \%$ & $4.792 \%$ \\
\hline 15 & $0.039 \%$ & $0.390 \%$ & $0.783 \%$ & $1.103 \%$ & $1.454 \%$ & $1.705 \%$ & $1.925 \%$ & $2.102 \%$ & $2.348 \%$ & $2.674 \%$ & $2.916 \%$ \\
\hline 16 & $0.038 \%$ & $0.378 \%$ & $0.743 \%$ & $1.248 \%$ & $1.755 \%$ & $2.645 \%$ & $3.656 \%$ & $4.546 \%$ & $5.019 \%$ & $5.219 \%$ & $5.502 \%$ \\
\hline 17 & $0.046 \%$ & $0.458 \%$ & $0.920 \%$ & $1.073 \%$ & $1.685 \%$ & $1.683 \%$ & $2.063 \%$ & $2.445 \%$ & $2.762 \%$ & $3.062 \%$ & $3.680 \%$ \\
\hline 18 & $0.044 \%$ & $0.445 \%$ & $0.887 \%$ & $1.281 \%$ & $1.770 \%$ & $2.223 \%$ & $2.638 \%$ & $3.041 \%$ & $3.331 \%$ & $3.681 \%$ & $3.990 \%$ \\
\hline 19 & $0.041 \%$ & $0.414 \%$ & $0.819 \%$ & $1.140 \%$ & $1.394 \%$ & $1.855 \%$ & $2.218 \%$ & $2.564 \%$ & $2.814 \%$ & $3.010 \%$ & $3.215 \%$ \\
\hline 20 & $0.043 \%$ & $0.428 \%$ & $0.826 \%$ & $1.253 \%$ & $1.809 \%$ & $2.149 \%$ & $2.413 \%$ & $2.668 \%$ & $2.866 \%$ & $3.039 \%$ & $3.381 \%$ \\
\hline 21 & $0.040 \%$ & $0.405 \%$ & $0.826 \%$ & $1.255 \%$ & $1.573 \%$ & $1.832 \%$ & $2.146 \%$ & $2.396 \%$ & $2.618 \%$ & $2.902 \%$ & $3.246 \%$ \\
\hline 22 & $0.043 \%$ & $0.428 \%$ & $0.858 \%$ & $1.343 \%$ & $1.686 \%$ & $2.080 \%$ & $2.570 \%$ & $3.026 \%$ & $3.436 \%$ & $3.863 \%$ & $4.250 \%$ \\
\hline
\end{tabular}

*This ground motion was disqualified for reasons discussed in Appendix B. 
Table D.1 cont'd: 3-Story drifts for load eccentricity and intensity

\begin{tabular}{|c|c|c|c|c|c|c|c|c|c|c|}
\hline $\begin{array}{l}\text { Ground } \\
\text { Motion }\end{array}$ & $\begin{array}{l}\text { PSa (g) } \\
1.1\end{array}$ & 1.2 & 1.3 & 1.4 & 1.5 & 1.6 & 1.7 & 1.8 & 1.9 & 2.0 \\
\hline 1 & $3.105 \%$ & $3.515 \%$ & $3.910 \%$ & $4.243 \%$ & $4.587 \%$ & $4.936 \%$ & $5.258 \%$ & $5.625 \%$ & $5.966 \%$ & $6.275 \%$ \\
\hline 2 & $3.260 \%$ & $3.481 \%$ & $3.833 \%$ & $4.421 \%$ & $5.091 \%$ & $5.496 \%$ & $5.933 \%$ & $6.290 \%$ & $6.602 \%$ & $6.841 \%$ \\
\hline 3 & $2.929 \%$ & $3.070 \%$ & $3.205 \%$ & $3.324 \%$ & $3.441 \%$ & $3.646 \%$ & $3.661 \%$ & $3.827 \%$ & $3.980 \%$ & $4.090 \%$ \\
\hline 4 & $4.508 \%$ & $4.849 \%$ & $5.117 \%$ & $5.372 \%$ & $5.585 \%$ & $5.784 \%$ & $6.072 \%$ & $6.617 \%$ & $6.898 \%$ & $7.227 \%$ \\
\hline 5 & $3.882 \%$ & $4.339 \%$ & $4.824 \%$ & $5.376 \%$ & $5.920 \%$ & $6.468 \%$ & $7.033 \%$ & $7.602 \%$ & $8.217 \%$ & $8.838 \%$ \\
\hline 6 & $5.422 \%$ & $6.306 \%$ & $7.315 \%$ & $8.282 \%$ & $9.136 \%$ & $9.911 \%$ & $10.703 \%$ & $11.394 \%$ & $11.992 \%$ & $12.880 \%$ \\
\hline 7 & $4.592 \%$ & $5.131 \%$ & $5.644 \%$ & $6.438 \%$ & $7.391 \%$ & $8.232 \%$ & $8.982 \%$ & $9.411 \%$ & $9.782 \%$ & $10.418 \%$ \\
\hline 8 & $5.119 \%$ & $5.799 \%$ & $6.558 \%$ & $7.305 \%$ & $7.920 \%$ & $8.484 \%$ & $8.985 \%$ & $9.544 \%$ & $10.117 \%$ & $10.682 \%$ \\
\hline 9 & $4.633 \%$ & $5.063 \%$ & $5.498 \%$ & $6.231 \%$ & $6.693 \%$ & $7.328 \%$ & $7.956 \%$ & $8.595 \%$ & $9.114 \%$ & $9.651 \%$ \\
\hline $10^{*}$ & ---- & ---- & ---- & ---- & ---- & ---- & ---- & ---- & ---- & ---- \\
\hline 11 & $3.538 \%$ & $4.255 \%$ & $4.959 \%$ & $5.788 \%$ & $6.518 \%$ & $7.163 \%$ & $7.836 \%$ & $8.560 \%$ & $9.165 \%$ & $9.709 \%$ \\
\hline 12 & $6.134 \%$ & $6.522 \%$ & $6.965 \%$ & $7.325 \%$ & $7.658 \%$ & $7.997 \%$ & $8.282 \%$ & $8.637 \%$ & $8.938 \%$ & $9.165 \%$ \\
\hline 13 & $4.099 \%$ & $4.376 \%$ & $4.803 \%$ & $5.417 \%$ & $5.237 \%$ & $5.127 \%$ & $5.304 \%$ & $5.554 \%$ & $5.772 \%$ & $6.371 \%$ \\
\hline 14 & $5.307 \%$ & $6.026 \%$ & $6.696 \%$ & $7.338 \%$ & $7.905 \%$ & $8.529 \%$ & $9.121 \%$ & $9.701 \%$ & $10.319 \%$ & $10.845 \%$ \\
\hline 15 & $3.260 \%$ & $3.612 \%$ & $3.810 \%$ & $4.072 \%$ & $4.438 \%$ & $4.699 \%$ & $5.024 \%$ & $5.340 \%$ & $5.786 \%$ & $6.245 \%$ \\
\hline 16 & $5.739 \%$ & $6.569 \%$ & $7.295 \%$ & $8.079 \%$ & $8.723 \%$ & $9.278 \%$ & $9.886 \%$ & $10.352 \%$ & $11.250 \%$ & $12.046 \%$ \\
\hline 17 & $3.894 \%$ & $4.235 \%$ & $4.691 \%$ & $5.156 \%$ & $5.657 \%$ & $6.179 \%$ & $6.718 \%$ & $7.122 \%$ & $7.698 \%$ & $8.240 \%$ \\
\hline 18 & $4.232 \%$ & $4.454 \%$ & $4.618 \%$ & $4.790 \%$ & $4.998 \%$ & $5.127 \%$ & $5.127 \%$ & $5.289 \%$ & $5.501 \%$ & $5.756 \%$ \\
\hline 19 & $3.367 \%$ & $3.626 \%$ & $4.057 \%$ & $4.340 \%$ & $4.657 \%$ & $4.973 \%$ & $5.389 \%$ & $5.601 \%$ & $5.924 \%$ & $6.284 \%$ \\
\hline 20 & $3.811 \%$ & $3.675 \%$ & $4.105 \%$ & $4.588 \%$ & $5.041 \%$ & $5.549 \%$ & $6.024 \%$ & $6.569 \%$ & $7.035 \%$ & $7.668 \%$ \\
\hline 21 & $3.634 \%$ & $4.108 \%$ & $4.627 \%$ & $5.106 \%$ & $5.596 \%$ & $6.106 \%$ & $6.673 \%$ & $7.259 \%$ & $7.559 \%$ & $8.051 \%$ \\
\hline 22 & $4.668 \%$ & $5.043 \%$ & $5.392 \%$ & $5.726 \%$ & $6.001 \%$ & $6.302 \%$ & $6.616 \%$ & $6.788 \%$ & $7.077 \%$ & $7.283 \%$ \\
\hline
\end{tabular}

*This ground motion was disqualified for reasons discussed in Appendix B. 
Table D.2: 3-Story drifts for load eccentricity only

\begin{tabular}{|c|c|c|c|c|c|c|c|c|c|c|c|}
\hline $\begin{array}{l}\text { Ground } \\
\text { Motion }\end{array}$ & $\begin{array}{l}\mathrm{PSa}(\mathrm{g}) \\
0.01\end{array}$ & 0.1 & 0.2 & 0.3 & 0.4 & 0.5 & 0.6 & 0.7 & 0.8 & 0.9 & 1.0 \\
\hline 1 & $0.049 \%$ & $0.485 \%$ & $0.875 \%$ & $1.089 \%$ & $1.375 \%$ & $1.673 \%$ & $1.968 \%$ & $2.218 \%$ & $2.370 \%$ & $2.437 \%$ & $2.712 \%$ \\
\hline 2 & $0.040 \%$ & $0.401 \%$ & $0.800 \%$ & $1.097 \%$ & $1.506 \%$ & $1.861 \%$ & $2.116 \%$ & $2.367 \%$ & $2.616 \%$ & $2.849 \%$ & $3.054 \%$ \\
\hline 3 & $0.039 \%$ & $0.386 \%$ & $0.772 \%$ & $1.064 \%$ & $1.383 \%$ & $1.816 \%$ & $2.127 \%$ & $2.341 \%$ & $2.493 \%$ & $2.612 \%$ & $2.735 \%$ \\
\hline 4 & $0.047 \%$ & $0.469 \%$ & $0.908 \%$ & $1.339 \%$ & $1.594 \%$ & $2.075 \%$ & $2.509 \%$ & $2.963 \%$ & $3.388 \%$ & $3.781 \%$ & $4.138 \%$ \\
\hline 5 & $0.052 \%$ & $0.519 \%$ & $0.953 \%$ & $1.273 \%$ & $1.581 \%$ & $1.891 \%$ & $2.220 \%$ & $2.552 \%$ & $2.880 \%$ & $3.220 \%$ & $3.573 \%$ \\
\hline 6 & $0.046 \%$ & $0.444 \%$ & $0.893 \%$ & $1.333 \%$ & $2.028 \%$ & $2.779 \%$ & $3.444 \%$ & $3.980 \%$ & $4.314 \%$ & $4.479 \%$ & $4.587 \%$ \\
\hline 7 & $0.058 \%$ & $0.575 \%$ & $1.142 \%$ & $1.516 \%$ & $2.128 \%$ & $2.559 \%$ & $2.942 \%$ & $3.347 \%$ & $3.811 \%$ & $4.131 \%$ & $4.419 \%$ \\
\hline 8 & $0.034 \%$ & $0.344 \%$ & $0.689 \%$ & $1.219 \%$ & $2.122 \%$ & $2.863 \%$ & $3.243 \%$ & $3.372 \%$ & $3.207 \%$ & $3.797 \%$ & $4.575 \%$ \\
\hline 9 & $0.043 \%$ & $0.433 \%$ & $0.856 \%$ & $1.156 \%$ & $1.375 \%$ & $1.931 \%$ & $2.449 \%$ & $2.925 \%$ & $3.382 \%$ & $3.819 \%$ & $4.252 \%$ \\
\hline $10^{*}$ & ---- & ---- & ---- & ---- & ---- & ---- & ---- & ---- & ---- & ---- & ---- \\
\hline 11 & $0.041 \%$ & $0.408 \%$ & $0.817 \%$ & $1.206 \%$ & $1.398 \%$ & $1.583 \%$ & $1.751 \%$ & $1.895 \%$ & $2.032 \%$ & $2.109 \%$ & $2.775 \%$ \\
\hline 12 & $0.053 \%$ & $0.526 \%$ & $1.083 \%$ & $1.404 \%$ & $1.718 \%$ & $2.225 \%$ & $3.135 \%$ & $3.841 \%$ & $4.509 \%$ & $5.056 \%$ & $5.554 \%$ \\
\hline 13 & $0.043 \%$ & $0.433 \%$ & $0.863 \%$ & $1.265 \%$ & $1.644 \%$ & $2.050 \%$ & $2.349 \%$ & $2.603 \%$ & $3.061 \%$ & $3.482 \%$ & $3.820 \%$ \\
\hline 14 & $0.047 \%$ & $0.469 \%$ & $0.935 \%$ & $1.302 \%$ & $1.909 \%$ & $2.519 \%$ & $2.976 \%$ & $3.379 \%$ & $3.739 \%$ & $4.084 \%$ & $4.627 \%$ \\
\hline 15 & $0.039 \%$ & $0.386 \%$ & $0.769 \%$ & $1.184 \%$ & $1.571 \%$ & $1.832 \%$ & $2.025 \%$ & $2.155 \%$ & $2.270 \%$ & $2.500 \%$ & $2.892 \%$ \\
\hline 16 & $0.039 \%$ & $0.390 \%$ & $0.777 \%$ & $1.106 \%$ & $1.722 \%$ & $2.781 \%$ & $3.835 \%$ & $4.726 \%$ & $5.049 \%$ & $5.225 \%$ & $5.440 \%$ \\
\hline 17 & $0.047 \%$ & $0.466 \%$ & $0.907 \%$ & $1.050 \%$ & $1.309 \%$ & $1.736 \%$ & $2.164 \%$ & $2.509 \%$ & $2.793 \%$ & $3.070 \%$ & $3.501 \%$ \\
\hline 18 & $0.047 \%$ & $0.471 \%$ & $0.940 \%$ & $1.374 \%$ & $1.860 \%$ & $2.330 \%$ & $2.742 \%$ & $3.138 \%$ & $3.464 \%$ & $3.780 \%$ & $4.064 \%$ \\
\hline 19 & $0.041 \%$ & $0.414 \%$ & $0.834 \%$ & $1.157 \%$ & $1.391 \%$ & $1.881 \%$ & $2.322 \%$ & $2.665 \%$ & $2.927 \%$ & $3.157 \%$ & $3.386 \%$ \\
\hline 20 & $0.037 \%$ & $0.375 \%$ & $0.745 \%$ & $1.060 \%$ & $1.503 \%$ & $1.916 \%$ & $2.236 \%$ & $2.511 \%$ & $2.759 \%$ & $2.998 \%$ & $3.220 \%$ \\
\hline 21 & $0.042 \%$ & $0.421 \%$ & $0.856 \%$ & $1.320 \%$ & $1.649 \%$ & $1.909 \%$ & $2.146 \%$ & $2.386 \%$ & $2.678 \%$ & $2.965 \%$ & $3.252 \%$ \\
\hline 22 & $0.041 \%$ & $0.413 \%$ & $0.827 \%$ & $1.302 \%$ & $1.649 \%$ & $2.099 \%$ & $2.588 \%$ & $3.043 \%$ & $3.466 \%$ & $3.878 \%$ & $4.287 \%$ \\
\hline
\end{tabular}

*This ground motion was disqualified for reasons discussed in Appendix B. 
Table D. 2 cont'd: 3-Story drifts for load eccentricity only

\begin{tabular}{|c|c|c|c|c|c|c|c|c|c|c|}
\hline $\begin{array}{l}\text { Ground } \\
\text { Motion }\end{array}$ & $\begin{array}{l}\text { PSa (g) } \\
1.1\end{array}$ & 1.2 & 1.3 & 1.4 & 1.5 & 1.6 & 1.7 & 1.8 & 1.9 & 2.0 \\
\hline 1 & $3.108 \%$ & $3.488 \%$ & $3.876 \%$ & $4.249 \%$ & $4.606 \%$ & $4.952 \%$ & $5.277 \%$ & $5.622 \%$ & $5.955 \%$ & $6.281 \%$ \\
\hline 2 & $3.187 \%$ & $3.419 \%$ & $3.865 \%$ & $4.421 \%$ & $5.000 \%$ & $5.447 \%$ & $5.974 \%$ & $6.404 \%$ & $6.745 \%$ & $6.973 \%$ \\
\hline 3 & $2.847 \%$ & $2.960 \%$ & $3.053 \%$ & $3.157 \%$ & $3.264 \%$ & $3.383 \%$ & $3.498 \%$ & $3.708 \%$ & $3.902 \%$ & $4.077 \%$ \\
\hline 4 & $4.474 \%$ & $4.791 \%$ & $5.076 \%$ & $5.351 \%$ & $5.619 \%$ & $5.871 \%$ & $6.101 \%$ & $6.424 \%$ & $6.803 \%$ & $7.189 \%$ \\
\hline 5 & $3.978 \%$ & $4.425 \%$ & $4.927 \%$ & $5.449 \%$ & $5.943 \%$ & $6.451 \%$ & $6.959 \%$ & $7.508 \%$ & $8.121 \%$ & $8.756 \%$ \\
\hline 6 & $5.417 \%$ & $6.370 \%$ & $7.410 \%$ & $8.408 \%$ & $9.284 \%$ & $10.061 \%$ & $10.686 \%$ & $11.456 \%$ & $12.229 \%$ & $12.914 \%$ \\
\hline 7 & $4.673 \%$ & $4.977 \%$ & $5.682 \%$ & $6.463 \%$ & $7.379 \%$ & $8.230 \%$ & $8.918 \%$ & $9.494 \%$ & $10.040 \%$ & $10.490 \%$ \\
\hline 8 & $5.232 \%$ & $5.931 \%$ & $6.633 \%$ & $7.359 \%$ & $7.983 \%$ & $8.566 \%$ & $9.089 \%$ & $9.656 \%$ & $10.205 \%$ & $10.753 \%$ \\
\hline 9 & $4.680 \%$ & $5.112 \%$ & $5.557 \%$ & $6.147 \%$ & $6.718 \%$ & $7.352 \%$ & $7.978 \%$ & $8.594 \%$ & $9.164 \%$ & $9.708 \%$ \\
\hline $10 *$ & ---- & ---- & ---- & ---- & ---- & ---- & ---- & ---- & ---- & ---- \\
\hline 11 & $3.619 \%$ & $4.430 \%$ & $5.221 \%$ & $6.009 \%$ & $6.765 \%$ & $7.470 \%$ & $8.113 \%$ & $8.696 \%$ & $9.242 \%$ & $9.792 \%$ \\
\hline 12 & $6.003 \%$ & $6.420 \%$ & $6.863 \%$ & $7.279 \%$ & $7.675 \%$ & $8.035 \%$ & $8.400 \%$ & $8.723 \%$ & $8.977 \%$ & $9.205 \%$ \\
\hline 13 & $4.107 \%$ & $4.333 \%$ & $4.510 \%$ & $4.739 \%$ & $4.927 \%$ & $5.283 \%$ & $5.544 \%$ & $5.766 \%$ & $5.934 \%$ & $6.167 \%$ \\
\hline 14 & $5.297 \%$ & $6.010 \%$ & $6.729 \%$ & $7.419 \%$ & $8.016 \%$ & $8.581 \%$ & $9.100 \%$ & $9.717 \%$ & $10.362 \%$ & $10.946 \%$ \\
\hline 15 & $3.288 \%$ & $3.655 \%$ & $3.973 \%$ & $4.215 \%$ & $4.420 \%$ & $4.696 \%$ & $5.071 \%$ & $5.427 \%$ & $5.817 \%$ & $6.192 \%$ \\
\hline 16 & $5.886 \%$ & $6.763 \%$ & $7.618 \%$ & $8.305 \%$ & $8.866 \%$ & $9.343 \%$ & $9.829 \%$ & $10.329 \%$ & $10.817 \%$ & $11.606 \%$ \\
\hline 17 & $3.977 \%$ & $4.406 \%$ & $4.870 \%$ & $5.346 \%$ & $5.826 \%$ & $6.315 \%$ & $6.793 \%$ & $7.227 \%$ & $7.690 \%$ & $8.189 \%$ \\
\hline 18 & $4.309 \%$ & $4.554 \%$ & $4.727 \%$ & $4.843 \%$ & $4.980 \%$ & $5.084 \%$ & $5.188 \%$ & $5.326 \%$ & $5.583 \%$ & $5.818 \%$ \\
\hline 19 & $3.603 \%$ & $3.764 \%$ & $4.087 \%$ & $4.434 \%$ & $4.765 \%$ & $5.059 \%$ & $5.384 \%$ & $5.627 \%$ & $5.933 \%$ & $6.280 \%$ \\
\hline 20 & $3.438 \%$ & $3.578 \%$ & $4.022 \%$ & $4.498 \%$ & $4.968 \%$ & $5.468 \%$ & $5.961 \%$ & $6.470 \%$ & $6.958 \%$ & $7.491 \%$ \\
\hline 21 & $3.561 \%$ & $4.076 \%$ & $4.601 \%$ & $5.134 \%$ & $5.688 \%$ & $6.226 \%$ & $6.699 \%$ & $7.207 \%$ & $7.563 \%$ & $7.953 \%$ \\
\hline 22 & $4.693 \%$ & $5.086 \%$ & $5.446 \%$ & $5.769 \%$ & $6.078 \%$ & $6.360 \%$ & $6.666 \%$ & $6.888 \%$ & $7.153 \%$ & $7.386 \%$ \\
\hline
\end{tabular}

*This ground motion was disqualified for reasons discussed in Appendix B. 
Table D.3: 3-Story drifts for load intensity only

\begin{tabular}{|c|c|c|c|c|c|c|c|c|c|c|c|}
\hline $\begin{array}{l}\text { Ground } \\
\text { Motion }\end{array}$ & $\begin{array}{l}\mathrm{PSa}(\mathrm{g}) \\
0.01\end{array}$ & 0.1 & 0.2 & 0.3 & 0.4 & 0.5 & 0.6 & 0.7 & 0.8 & 0.9 & 1.0 \\
\hline 1 & $0.041 \%$ & $0.410 \%$ & $0.811 \%$ & $1.064 \%$ & $1.341 \%$ & $1.632 \%$ & $1.869 \%$ & $2.016 \%$ & $2.141 \%$ & $2.399 \%$ & $2.633 \%$ \\
\hline 2 & $0.038 \%$ & $0.381 \%$ & $0.764 \%$ & $1.078 \%$ & $1.612 \%$ & $1.879 \%$ & $2.136 \%$ & $2.372 \%$ & $2.579 \%$ & $2.781 \%$ & $2.966 \%$ \\
\hline 3 & $0.038 \%$ & $0.379 \%$ & $0.758 \%$ & $1.035 \%$ & $1.328 \%$ & $1.750 \%$ & $2.073 \%$ & $2.302 \%$ & $2.480 \%$ & $2.634 \%$ & $2.781 \%$ \\
\hline 4 & $0.049 \%$ & $0.488 \%$ & $1.008 \%$ & $1.081 \%$ & $1.770 \%$ & $1.989 \%$ & $2.439 \%$ & $2.886 \%$ & $3.324 \%$ & $3.725 \%$ & $4.117 \%$ \\
\hline 5 & $0.037 \%$ & $0.374 \%$ & $0.776 \%$ & $1.070 \%$ & $1.416 \%$ & $1.769 \%$ & $2.106 \%$ & $2.513 \%$ & $2.908 \%$ & $3.213 \%$ & $3.518 \%$ \\
\hline 6 & $0.053 \%$ & $0.513 \%$ & $0.976 \%$ & $1.524 \%$ & $2.011 \%$ & $2.750 \%$ & $3.344 \%$ & $3.797 \%$ & $4.005 \%$ & $4.171 \%$ & $4.378 \%$ \\
\hline 7 & $0.051 \%$ & $0.514 \%$ & $0.968 \%$ & $2.043 \%$ & $2.064 \%$ & $2.430 \%$ & $2.894 \%$ & $3.283 \%$ & $3.606 \%$ & $4.117 \%$ & $4.466 \%$ \\
\hline 8 & $0.038 \%$ & $0.377 \%$ & $0.754 \%$ & $1.259 \%$ & $2.127 \%$ & $2.700 \%$ & $3.086 \%$ & $3.207 \%$ & $3.733 \%$ & $3.786 \%$ & $4.361 \%$ \\
\hline 9 & $0.042 \%$ & $0.418 \%$ & $0.818 \%$ & $1.063 \%$ & $1.399 \%$ & $1.838 \%$ & $2.341 \%$ & $2.821 \%$ & $3.291 \%$ & $3.741 \%$ & $4.189 \%$ \\
\hline $10^{*}$ & ---- & ---- & ---- & ---- & ---- & ---- & ---- & ---- & ---- & ---- & ---- \\
\hline 11 & $0.036 \%$ & $0.361 \%$ & $0.724 \%$ & $1.058 \%$ & $1.303 \%$ & $1.462 \%$ & $1.607 \%$ & $1.867 \%$ & $2.046 \%$ & $2.510 \%$ & $2.875 \%$ \\
\hline 12 & $0.052 \%$ & $0.523 \%$ & $1.070 \%$ & $1.621 \%$ & $1.950 \%$ & $2.482 \%$ & $3.326 \%$ & $3.946 \%$ & $4.592 \%$ & $5.208 \%$ & $5.660 \%$ \\
\hline 13 & $0.053 \%$ & $0.529 \%$ & $1.110 \%$ & $1.476 \%$ & $1.751 \%$ & $2.217 \%$ & $2.426 \%$ & $2.759 \%$ & $3.027 \%$ & $3.461 \%$ & $3.841 \%$ \\
\hline 14 & $0.046 \%$ & $0.463 \%$ & $0.901 \%$ & $1.294 \%$ & $1.870 \%$ & $2.449 \%$ & $2.925 \%$ & $3.348 \%$ & $3.717 \%$ & $4.085 \%$ & $4.634 \%$ \\
\hline 15 & $0.038 \%$ & $0.381 \%$ & $0.763 \%$ & $1.118 \%$ & $1.456 \%$ & $1.728 \%$ & $1.937 \%$ & $2.118 \%$ & $2.362 \%$ & $2.662 \%$ & $2.914 \%$ \\
\hline 16 & $0.039 \%$ & $0.387 \%$ & $0.760 \%$ & $1.156 \%$ & $1.737 \%$ & $2.660 \%$ & $3.640 \%$ & $4.494 \%$ & $5.009 \%$ & $5.232 \%$ & $5.435 \%$ \\
\hline 17 & $0.043 \%$ & $0.430 \%$ & $0.840 \%$ & $1.038 \%$ & $1.552 \%$ & $1.634 \%$ & $2.036 \%$ & $2.442 \%$ & $2.763 \%$ & $3.031 \%$ & $3.455 \%$ \\
\hline 18 & $0.045 \%$ & $0.452 \%$ & $0.904 \%$ & $1.297 \%$ & $1.787 \%$ & $2.235 \%$ & $2.617 \%$ & $2.985 \%$ & $3.317 \%$ & $3.645 \%$ & $3.971 \%$ \\
\hline 19 & $0.041 \%$ & $0.411 \%$ & $0.809 \%$ & $1.125 \%$ & $1.391 \%$ & $1.854 \%$ & $2.250 \%$ & $2.591 \%$ & $2.855 \%$ & $3.042 \%$ & $3.226 \%$ \\
\hline 20 & $0.042 \%$ & $0.418 \%$ & $0.810 \%$ & $1.198 \%$ & $1.727 \%$ & $2.103 \%$ & $2.377 \%$ & $2.613 \%$ & $2.794 \%$ & $2.922 \%$ & $3.169 \%$ \\
\hline 21 & $0.040 \%$ & $0.403 \%$ & $0.811 \%$ & $1.262 \%$ & $1.587 \%$ & $1.846 \%$ & $2.126 \%$ & $2.408 \%$ & $2.632 \%$ & $2.901 \%$ & $3.220 \%$ \\
\hline 22 & $0.042 \%$ & $0.415 \%$ & $0.833 \%$ & $1.259 \%$ & $1.652 \%$ & $2.083 \%$ & $2.575 \%$ & $3.033 \%$ & $3.443 \%$ & $3.865 \%$ & $4.257 \%$ \\
\hline
\end{tabular}

*This ground motion was disqualified for reasons discussed in Appendix B. 
Table D.3 cont'd: 3-Story drifts for load intensity only

\begin{tabular}{|c|c|c|c|c|c|c|c|c|c|c|}
\hline $\begin{array}{l}\text { Ground } \\
\text { Motion }\end{array}$ & $\begin{array}{l}\text { PSa (g) } \\
1.1\end{array}$ & 1.2 & 1.3 & 1.4 & 1.5 & 1.6 & 1.7 & 1.8 & 1.9 & 2.0 \\
\hline 1 & $3.081 \%$ & $3.488 \%$ & $3.875 \%$ & $4.232 \%$ & $4.593 \%$ & $4.935 \%$ & $5.264 \%$ & $5.598 \%$ & $5.930 \%$ & $6.272 \%$ \\
\hline 2 & $3.268 \%$ & $3.480 \%$ & $3.833 \%$ & $4.341 \%$ & $4.943 \%$ & $5.479 \%$ & $5.940 \%$ & $6.302 \%$ & $6.613 \%$ & $6.845 \%$ \\
\hline 3 & $2.917 \%$ & $3.044 \%$ & $3.187 \%$ & $3.304 \%$ & $3.408 \%$ & $3.581 \%$ & $3.655 \%$ & $3.784 \%$ & $3.928 \%$ & $4.061 \%$ \\
\hline 4 & $4.486 \%$ & $4.815 \%$ & $5.094 \%$ & $5.355 \%$ & $5.589 \%$ & $5.789 \%$ & $6.080 \%$ & $6.592 \%$ & $6.887 \%$ & $7.222 \%$ \\
\hline 5 & $3.876 \%$ & $4.338 \%$ & $4.822 \%$ & $5.337 \%$ & $5.879 \%$ & $6.408 \%$ & $6.972 \%$ & $7.527 \%$ & $8.115 \%$ & $8.732 \%$ \\
\hline 6 & $5.283 \%$ & $6.276 \%$ & $7.314 \%$ & $8.287 \%$ & $9.153 \%$ & $9.923 \%$ & $10.647 \%$ & $11.379 \%$ & $11.997 \%$ & $12.807 \%$ \\
\hline 7 & $4.624 \%$ & $5.109 \%$ & $5.607 \%$ & $6.354 \%$ & $7.162 \%$ & $7.990 \%$ & $8.723 \%$ & $9.266 \%$ & $9.766 \%$ & $10.379 \%$ \\
\hline 8 & $4.992 \%$ & $5.622 \%$ & $6.298 \%$ & $6.942 \%$ & $7.555 \%$ & $8.157 \%$ & $8.764 \%$ & $9.343 \%$ & $9.957 \%$ & $10.508 \%$ \\
\hline 9 & $4.630 \%$ & $5.070 \%$ & $5.507 \%$ & $6.074 \%$ & $6.592 \%$ & $7.217 \%$ & $7.863 \%$ & $8.504 \%$ & $9.065 \%$ & $9.601 \%$ \\
\hline $10 *$ & ---- & ---- & ---- & ---- & ---- & ---- & ---- & ---- & ---- & ---- \\
\hline 11 & $3.376 \%$ & $4.121 \%$ & $4.866 \%$ & $5.691 \%$ & $6.461 \%$ & $7.175 \%$ & $7.871 \%$ & $8.576 \%$ & $9.183 \%$ & $9.731 \%$ \\
\hline 12 & $6.120 \%$ & $6.533 \%$ & $6.952 \%$ & $7.321 \%$ & $7.666 \%$ & $8.015 \%$ & $8.283 \%$ & $8.584 \%$ & $8.856 \%$ & $9.034 \%$ \\
\hline 13 & $4.097 \%$ & $4.355 \%$ & $4.706 \%$ & $5.167 \%$ & $5.110 \%$ & $5.097 \%$ & $5.366 \%$ & $5.604 \%$ & $5.800 \%$ & $6.261 \%$ \\
\hline 14 & $5.200 \%$ & $5.904 \%$ & $6.601 \%$ & $7.247 \%$ & $7.874 \%$ & $8.505 \%$ & $9.084 \%$ & $9.684 \%$ & $10.306 \%$ & $10.854 \%$ \\
\hline 15 & $3.268 \%$ & $3.619 \%$ & $3.831 \%$ & $4.082 \%$ & $4.425 \%$ & $4.690 \%$ & $5.010 \%$ & $5.337 \%$ & $5.751 \%$ & $6.197 \%$ \\
\hline 16 & $5.724 \%$ & $6.576 \%$ & $7.308 \%$ & $8.063 \%$ & $8.718 \%$ & $9.299 \%$ & $9.883 \%$ & $10.356 \%$ & $11.061 \%$ & $11.752 \%$ \\
\hline 17 & $3.743 \%$ & $4.179 \%$ & $4.687 \%$ & $5.185 \%$ & $5.690 \%$ & $6.209 \%$ & $6.734 \%$ & $7.187 \%$ & $7.699 \%$ & $8.196 \%$ \\
\hline 18 & $4.242 \%$ & $4.463 \%$ & $4.637 \%$ & $4.790 \%$ & $4.938 \%$ & $5.043 \%$ & $5.129 \%$ & $5.285 \%$ & $5.515 \%$ & $5.765 \%$ \\
\hline 19 & $3.377 \%$ & $3.636 \%$ & $4.009 \%$ & $4.271 \%$ & $4.597 \%$ & $4.930 \%$ & $5.276 \%$ & $5.571 \%$ & $5.920 \%$ & $6.295 \%$ \\
\hline 20 & $3.455 \%$ & $3.552 \%$ & $3.990 \%$ & $4.455 \%$ & $4.919 \%$ & $5.412 \%$ & $5.890 \%$ & $6.406 \%$ & $6.869 \%$ & $7.429 \%$ \\
\hline 21 & $3.629 \%$ & $4.087 \%$ & $4.612 \%$ & $5.114 \%$ & $5.606 \%$ & $6.107 \%$ & $6.630 \%$ & $7.131 \%$ & $7.526 \%$ & $8.034 \%$ \\
\hline 22 & $4.671 \%$ & $5.047 \%$ & $5.397 \%$ & $5.735 \%$ & $6.012 \%$ & $6.319 \%$ & $6.598 \%$ & $6.822 \%$ & $7.090 \%$ & $7.305 \%$ \\
\hline
\end{tabular}

*This ground motion was disqualified for reasons discussed in Appendix B. 
Table D.4: 3-Story drifts for mean load

\begin{tabular}{|c|c|c|c|c|c|c|c|c|c|c|c|}
\hline $\begin{array}{l}\text { Ground } \\
\text { Motion }\end{array}$ & $\begin{array}{l}\mathrm{PSa}(\mathrm{g}) \\
0.01\end{array}$ & 0.1 & 0.2 & 0.3 & 0.4 & 0.5 & 0.6 & 0.7 & 0.8 & 0.9 & 1.0 \\
\hline 1 & $0.043 \%$ & $0.430 \%$ & $0.836 \%$ & $1.071 \%$ & $1.309 \%$ & $1.679 \%$ & $1.991 \%$ & $2.240 \%$ & $2.378 \%$ & $2.442 \%$ & $2.679 \%$ \\
\hline 2 & $0.040 \%$ & $0.399 \%$ & $0.797 \%$ & $1.070 \%$ & $1.462 \%$ & $1.806 \%$ & $2.113 \%$ & $2.381 \%$ & $2.638 \%$ & $2.873 \%$ & $3.066 \%$ \\
\hline 3 & $0.038 \%$ & $0.379 \%$ & $0.759 \%$ & $1.053 \%$ & $1.323 \%$ & $1.786 \%$ & $2.094 \%$ & $2.307 \%$ & $2.472 \%$ & $2.604 \%$ & $2.726 \%$ \\
\hline 4 & $0.049 \%$ & $0.487 \%$ & $0.900 \%$ & $1.370 \%$ & $1.520 \%$ & $2.072 \%$ & $2.518 \%$ & $2.954 \%$ & $3.377 \%$ & $3.776 \%$ & $4.153 \%$ \\
\hline 5 & $0.050 \%$ & $0.498 \%$ & $0.912 \%$ & $1.232 \%$ & $1.539 \%$ & $1.868 \%$ & $2.224 \%$ & $2.559 \%$ & $2.881 \%$ & $3.218 \%$ & $3.581 \%$ \\
\hline 6 & $0.046 \%$ & $0.445 \%$ & $0.879 \%$ & $1.292 \%$ & $2.000 \%$ & $2.759 \%$ & $3.449 \%$ & $3.802 \%$ & $4.042 \%$ & $4.226 \%$ & $4.432 \%$ \\
\hline 7 & $0.059 \%$ & $0.587 \%$ & $1.175 \%$ & $1.539 \%$ & $2.149 \%$ & $2.555 \%$ & $2.945 \%$ & $3.359 \%$ & $3.821 \%$ & $4.118 \%$ & $4.419 \%$ \\
\hline 8 & $0.033 \%$ & $0.333 \%$ & $0.666 \%$ & $1.153 \%$ & $2.101 \%$ & $2.873 \%$ & $3.226 \%$ & $3.357 \%$ & $3.182 \%$ & $3.711 \%$ & $4.442 \%$ \\
\hline 9 & $0.043 \%$ & $0.427 \%$ & $0.846 \%$ & $1.087 \%$ & $1.328 \%$ & $1.870 \%$ & $2.393 \%$ & $2.880 \%$ & $3.347 \%$ & $3.794 \%$ & $4.236 \%$ \\
\hline $10^{*}$ & ---- & ---- & ---- & ---- & ---- & ---- & ---- & ---- & ---- & ---- & ---- \\
\hline 11 & $0.037 \%$ & $0.374 \%$ & $0.749 \%$ & $1.141 \%$ & $1.330 \%$ & $1.551 \%$ & $1.737 \%$ & $1.862 \%$ & $1.949 \%$ & $2.048 \%$ & $2.621 \%$ \\
\hline 12 & $0.054 \%$ & $0.535 \%$ & $0.973 \%$ & $1.289 \%$ & $1.677 \%$ & $2.175 \%$ & $2.993 \%$ & $3.825 \%$ & $4.515 \%$ & $5.049 \%$ & $5.539 \%$ \\
\hline 13 & $0.044 \%$ & $0.442 \%$ & $0.865 \%$ & $1.247 \%$ & $1.665 \%$ & $1.994 \%$ & $2.306 \%$ & $2.561 \%$ & $3.040 \%$ & $3.471 \%$ & $3.814 \%$ \\
\hline 14 & $0.048 \%$ & $0.479 \%$ & $0.950 \%$ & $1.282 \%$ & $1.883 \%$ & $2.492 \%$ & $2.980 \%$ & $3.380 \%$ & $3.752 \%$ & $4.089 \%$ & $4.528 \%$ \\
\hline 15 & $0.038 \%$ & $0.378 \%$ & $0.757 \%$ & $1.192 \%$ & $1.579 \%$ & $1.857 \%$ & $2.038 \%$ & $2.170 \%$ & $2.285 \%$ & $2.490 \%$ & $2.890 \%$ \\
\hline 16 & $0.039 \%$ & $0.390 \%$ & $0.776 \%$ & $0.994 \%$ & $1.703 \%$ & $2.808 \%$ & $3.819 \%$ & $4.672 \%$ & $5.064 \%$ & $5.239 \%$ & $5.374 \%$ \\
\hline 17 & $0.046 \%$ & $0.458 \%$ & $0.909 \%$ & $1.069 \%$ & $1.258 \%$ & $1.689 \%$ & $2.135 \%$ & $2.511 \%$ & $2.795 \%$ & $3.043 \%$ & $3.287 \%$ \\
\hline 18 & $0.048 \%$ & $0.479 \%$ & $0.958 \%$ & $1.391 \%$ & $1.880 \%$ & $2.341 \%$ & $2.725 \%$ & $3.074 \%$ & $3.425 \%$ & $3.744 \%$ & $4.034 \%$ \\
\hline 19 & $0.041 \%$ & $0.411 \%$ & $0.824 \%$ & $1.142 \%$ & $1.403 \%$ & $1.880 \%$ & $2.334 \%$ & $2.681 \%$ & $2.955 \%$ & $3.195 \%$ & $3.439 \%$ \\
\hline 20 & $0.038 \%$ & $0.379 \%$ & $0.757 \%$ & $1.045 \%$ & $1.434 \%$ & $1.873 \%$ & $2.202 \%$ & $2.468 \%$ & $2.698 \%$ & $2.884 \%$ & $3.017 \%$ \\
\hline 21 & $0.042 \%$ & $0.419 \%$ & $0.841 \%$ & $1.328 \%$ & $1.664 \%$ & $1.922 \%$ & $2.142 \%$ & $2.398 \%$ & $2.691 \%$ & $2.970 \%$ & $3.226 \%$ \\
\hline 22 & $0.040 \%$ & $0.400 \%$ & $0.803 \%$ & $1.220 \%$ & $1.593 \%$ & $2.103 \%$ & $2.593 \%$ & $3.049 \%$ & $3.472 \%$ & $3.880 \%$ & $4.294 \%$ \\
\hline
\end{tabular}

*This ground motion was disqualified for reasons discussed in Appendix B. 
Table D.4 cont'd: 3-Story drifts for mean load

\begin{tabular}{|c|c|c|c|c|c|c|c|c|c|c|}
\hline $\begin{array}{l}\text { Ground } \\
\text { Motion }\end{array}$ & $\begin{array}{l}\text { PSa (g) } \\
1.1\end{array}$ & 1.2 & 1.3 & 1.4 & 1.5 & 1.6 & 1.7 & 1.8 & 1.9 & 2.0 \\
\hline 1 & $3.084 \%$ & $3.481 \%$ & $3.874 \%$ & $4.258 \%$ & $4.612 \%$ & $4.951 \%$ & $5.283 \%$ & $5.612 \%$ & $5.946 \%$ & $6.278 \%$ \\
\hline 2 & $3.196 \%$ & $3.432 \%$ & $3.865 \%$ & $4.342 \%$ & $4.854 \%$ & $5.421 \%$ & $5.984 \%$ & $6.429 \%$ & $6.756 \%$ & $6.981 \%$ \\
\hline 3 & $2.836 \%$ & $2.934 \%$ & $3.036 \%$ & $3.138 \%$ & $3.233 \%$ & $3.325 \%$ & $3.488 \%$ & $3.677 \%$ & $3.866 \%$ & $4.061 \%$ \\
\hline 4 & $4.487 \%$ & $4.794 \%$ & $5.082 \%$ & $5.360 \%$ & $5.624 \%$ & $5.886 \%$ & $6.141 \%$ & $6.400 \%$ & $6.793 \%$ & $7.184 \%$ \\
\hline 5 & $3.987 \%$ & $4.434 \%$ & $4.925 \%$ & $5.409 \%$ & $5.888 \%$ & $6.375 \%$ & $6.880 \%$ & $7.426 \%$ & $8.013 \%$ & $8.641 \%$ \\
\hline 6 & $5.303 \%$ & $6.340 \%$ & $7.408 \%$ & $8.413 \%$ & $9.301 \%$ & $10.073 \%$ & $10.762 \%$ & $11.441 \%$ & $12.212 \%$ & $12.909 \%$ \\
\hline 7 & $4.706 \%$ & $4.946 \%$ & $5.645 \%$ & $6.380 \%$ & $7.174 \%$ & $7.988 \%$ & $8.778 \%$ & $9.466 \%$ & $10.024 \%$ & $10.434 \%$ \\
\hline 8 & $5.102 \%$ & $5.750 \%$ & $6.370 \%$ & $7.024 \%$ & $7.664 \%$ & $8.277 \%$ & $8.867 \%$ & $9.448 \%$ & $10.017 \%$ & $10.592 \%$ \\
\hline 9 & $4.676 \%$ & $5.120 \%$ & $5.566 \%$ & $6.008 \%$ & $6.649 \%$ & $7.263 \%$ & $7.877 \%$ & $8.508 \%$ & $9.119 \%$ & $9.658 \%$ \\
\hline $10^{*}$ & ---- & ---- & ---- & ---- & ---- & ---- & ---- & ---- & ---- & ---- \\
\hline 11 & $3.453 \%$ & $4.290 \%$ & $5.122 \%$ & $5.908 \%$ & $6.707 \%$ & $7.482 \%$ & $8.152 \%$ & $8.712 \%$ & $9.260 \%$ & $9.807 \%$ \\
\hline 12 & $5.990 \%$ & $6.430 \%$ & $6.850 \%$ & $7.275 \%$ & $7.683 \%$ & $8.054 \%$ & $8.402 \%$ & $8.669 \%$ & $8.896 \%$ & $9.073 \%$ \\
\hline 13 & $4.125 \%$ & $4.324 \%$ & $4.467 \%$ & $4.564 \%$ & $4.942 \%$ & $5.309 \%$ & $5.608 \%$ & $5.819 \%$ & $5.963 \%$ & $6.060 \%$ \\
\hline 14 & $5.190 \%$ & $5.889 \%$ & $6.617 \%$ & $7.328 \%$ & $7.987 \%$ & $8.563 \%$ & $9.072 \%$ & $9.726 \%$ & $10.348 \%$ & $10.942 \%$ \\
\hline 15 & $3.299 \%$ & $3.662 \%$ & $3.995 \%$ & $4.226 \%$ & $4.407 \%$ & $4.680 \%$ & $5.054 \%$ & $5.432 \%$ & $5.798 \%$ & $6.159 \%$ \\
\hline 16 & $5.862 \%$ & $6.788 \%$ & $7.648 \%$ & $8.319 \%$ & $8.863 \%$ & $9.366 \%$ & $9.831 \%$ & $10.354 \%$ & $10.836 \%$ & $11.271 \%$ \\
\hline 17 & $3.823 \%$ & $4.348 \%$ & $4.867 \%$ & $5.376 \%$ & $5.874 \%$ & $6.345 \%$ & $6.812 \%$ & $7.293 \%$ & $7.737 \%$ & $8.183 \%$ \\
\hline 18 & $4.315 \%$ & $4.564 \%$ & $4.746 \%$ & $4.850 \%$ & $4.918 \%$ & $5.035 \%$ & $5.190 \%$ & $5.347 \%$ & $5.597 \%$ & $5.827 \%$ \\
\hline 19 & $3.645 \%$ & $3.827 \%$ & $4.033 \%$ & $4.382 \%$ & $4.704 \%$ & $4.997 \%$ & $5.285 \%$ & $5.582 \%$ & $5.910 \%$ & $6.287 \%$ \\
\hline 20 & $3.117 \%$ & $3.458 \%$ & $3.909 \%$ & $4.367 \%$ & $4.848 \%$ & $5.332 \%$ & $5.828 \%$ & $6.310 \%$ & $6.793 \%$ & $7.259 \%$ \\
\hline 21 & $3.548 \%$ & $4.055 \%$ & $4.586 \%$ & $5.142 \%$ & $5.703 \%$ & $6.227 \%$ & $6.712 \%$ & $7.134 \%$ & $7.520 \%$ & $7.909 \%$ \\
\hline 22 & $4.696 \%$ & $5.089 \%$ & $5.451 \%$ & $5.778 \%$ & $6.089 \%$ & $6.378 \%$ & $6.649 \%$ & $6.923 \%$ & $7.167 \%$ & $7.408 \%$ \\
\hline
\end{tabular}

*This ground motion was disqualified for reasons discussed in Appendix B. 
Table D.5: 9-Story drifts for load eccentricity and intensity

\begin{tabular}{|c|c|c|c|c|c|c|c|c|c|c|c|}
\hline $\begin{array}{l}\text { Ground } \\
\text { Motion }\end{array}$ & $\begin{array}{l}\text { PSa (g) } \\
0.1\end{array}$ & 0.15 & 0.2 & 0.3 & 0.4 & 0.5 & 0.6 & 0.7 & 0.8 & 0.9 & 1.0 \\
\hline 1 & $1.753 \%$ & $2.873 \%$ & $2.407 \%$ & $3.118 \%$ & $3.811 \%$ & $4.820 \%$ & $4.969 \%$ & $6.467 \%$ & $14.409 \%$ & $8.277 \%$ & $6.544 \%$ \\
\hline 2 & $0.860 \%$ & $1.186 \%$ & $1.388 \%$ & $2.212 \%$ & $3.026 \%$ & $3.414 \%$ & $3.510 \%$ & $3.446 \%$ & $3.943 \%$ & $4.596 \%$ & $4.850 \%$ \\
\hline 3 & $1.515 \%$ & $2.352 \%$ & $2.940 \%$ & $3.648 \%$ & $4.216 \%$ & $4.478 \%$ & $4.730 \%$ & $5.058 \%$ & $5.361 \%$ & $5.591 \%$ & $7.507 \%$ \\
\hline 4 & $1.044 \%$ & $1.520 \%$ & $1.750 \%$ & $2.043 \%$ & $2.628 \%$ & $3.005 \%$ & $3.237 \%$ & $4.637 \%$ & $4.308 \%$ & $4.706 \%$ & $5.510 \%$ \\
\hline 5 & $1.372 \%$ & $2.053 \%$ & $1.897 \%$ & $2.829 \%$ & $3.158 \%$ & $3.631 \%$ & $4.134 \%$ & $9.557 \%$ & $10.707 \%$ & $5.824 \%$ & $8.156 \%$ \\
\hline 6 & $0.787 \%$ & $1.077 \%$ & $1.394 \%$ & $2.115 \%$ & $2.627 \%$ & $3.170 \%$ & $3.509 \%$ & $3.733 \%$ & $4.102 \%$ & $5.024 \%$ & $5.813 \%$ \\
\hline 7 & $1.143 \%$ & $1.701 \%$ & $2.027 \%$ & $2.397 \%$ & $2.523 \%$ & $3.004 \%$ & $3.358 \%$ & $15.387 \%$ & $13.841 \%$ & $5.829 \%$ & $4.454 \%$ \\
\hline 8 & $1.156 \%$ & $1.526 \%$ & $1.771 \%$ & $2.420 \%$ & $3.140 \%$ & $3.919 \%$ & $4.482 \%$ & $5.198 \%$ & $5.825 \%$ & $5.928 \%$ & $8.143 \%$ \\
\hline 9 & $0.856 \%$ & $1.131 \%$ & $1.527 \%$ & $2.121 \%$ & $2.651 \%$ & $3.245 \%$ & $3.902 \%$ & $4.562 \%$ & $5.295 \%$ & $6.639 \%$ & $7.320 \%$ \\
\hline $10^{*}$ & ---- & ---- & ---- & ---- & ---- & ---- & ---- & ---- & ---- & ---- & ---- \\
\hline 11 & $1.003 \%$ & $1.464 \%$ & $2.011 \%$ & $2.960 \%$ & $3.654 \%$ & $4.638 \%$ & $5.473 \%$ & $7.463 \%$ & $10.305 \%$ & $12.059 \%$ & $14.682 \%$ \\
\hline 12 & $2.382 \%$ & $3.068 \%$ & $2.907 \%$ & $3.709 \%$ & $3.592 \%$ & $4.357 \%$ & $5.074 \%$ & $6.303 \%$ & $7.855 \%$ & $9.218 \%$ & $10.261 \%$ \\
\hline 13 & $1.653 \%$ & $2.176 \%$ & $2.787 \%$ & $3.503 \%$ & $9.007 \%$ & $5.145 \%$ & $10.569 \%$ & $9.402 \%$ & $9.575 \%$ & $13.014 \%$ & $7.771 \%$ \\
\hline 14 & $0.748 \%$ & $1.113 \%$ & $1.292 \%$ & $1.695 \%$ & $2.073 \%$ & $2.375 \%$ & $2.575 \%$ & $2.853 \%$ & $3.323 \%$ & $3.788 \%$ & $4.273 \%$ \\
\hline 15 & $0.750 \%$ & $1.044 \%$ & $1.161 \%$ & $1.562 \%$ & $1.982 \%$ & $2.232 \%$ & $2.584 \%$ & $2.854 \%$ & $3.158 \%$ & $3.461 \%$ & $3.787 \%$ \\
\hline 16 & $0.717 \%$ & $0.957 \%$ & $1.326 \%$ & $2.024 \%$ & $2.470 \%$ & $2.749 \%$ & $2.946 \%$ & $3.342 \%$ & $3.591 \%$ & $4.688 \%$ & $4.608 \%$ \\
\hline 17 & $1.039 \%$ & $1.348 \%$ & $1.503 \%$ & $1.971 \%$ & $2.436 \%$ & $3.884 \%$ & $4.273 \%$ & $4.514 \%$ & $5.372 \%$ & $6.224 \%$ & $8.085 \%$ \\
\hline 18 & $1.429 \%$ & $2.351 \%$ & $3.012 \%$ & $3.346 \%$ & $3.681 \%$ & $4.683 \%$ & $8.231 \%$ & $5.670 \%$ & $6.003 \%$ & $7.684 \%$ & $9.027 \%$ \\
\hline 19 & $0.811 \%$ & $1.216 \%$ & $1.492 \%$ & $1.947 \%$ & $2.473 \%$ & $3.121 \%$ & $4.018 \%$ & $4.257 \%$ & $6.357 \%$ & $7.002 \%$ & $8.788 \%$ \\
\hline 20 & $1.351 \%$ & $2.069 \%$ & $2.447 \%$ & $2.716 \%$ & $3.213 \%$ & $3.784 \%$ & $5.654 \%$ & $7.358 \%$ & $9.096 \%$ & $11.306 \%$ & $16.370 \%$ \\
\hline 21 & $1.121 \%$ & $1.723 \%$ & $2.225 \%$ & $2.491 \%$ & $2.973 \%$ & $4.305 \%$ & $5.152 \%$ & $8.190 \%$ & $9.665 \%$ & $7.689 \%$ & $7.784 \%$ \\
\hline $22^{*}$ & ---- & ---- & ---- & ---- & ---- & ---- & ---- & ---- & ---- & ---- & ---- \\
\hline
\end{tabular}

\footnotetext{
*This ground motion was disqualified for reasons discussed in Appendix B.
} 
Table D.6: 9-Story drifts for load eccentricity only

\begin{tabular}{|c|c|c|c|c|c|c|c|c|c|c|c|}
\hline $\begin{array}{l}\text { Ground } \\
\text { Motion }\end{array}$ & $\begin{array}{l}\text { PSa (g) } \\
0.1\end{array}$ & 0.15 & 0.2 & 0.3 & 0.4 & 0.5 & 0.6 & 0.7 & 0.8 & 0.9 & 1.0 \\
\hline 1 & $1.551 \%$ & $2.190 \%$ & $2.439 \%$ & $2.924 \%$ & $3.493 \%$ & $4.774 \%$ & $5.075 \%$ & $5.742 \%$ & $11.110 \%$ & $8.966 \%$ & $6.467 \%$ \\
\hline 2 & $0.906 \%$ & $1.220 \%$ & $1.391 \%$ & $2.254 \%$ & $3.090 \%$ & $3.470 \%$ & $3.571 \%$ & $3.454 \%$ & $3.923 \%$ & $4.488 \%$ & $4.881 \%$ \\
\hline 3 & $1.510 \%$ & $2.385 \%$ & $2.969 \%$ & $3.684 \%$ & $4.212 \%$ & $4.510 \%$ & $4.716 \%$ & $5.102 \%$ & $5.155 \%$ & $5.490 \%$ & $6.677 \%$ \\
\hline 4 & $1.034 \%$ & $1.591 \%$ & $1.855 \%$ & $1.929 \%$ & $2.637 \%$ & $3.051 \%$ & $3.244 \%$ & $4.607 \%$ & $4.358 \%$ & $4.717 \%$ & $5.400 \%$ \\
\hline 5 & $1.027 \%$ & $1.687 \%$ & $2.014 \%$ & $2.922 \%$ & $3.266 \%$ & $3.648 \%$ & $4.185 \%$ & $7.066 \%$ & $7.630 \%$ & $5.416 \%$ & $5.879 \%$ \\
\hline 6 & $0.783 \%$ & $1.055 \%$ & $1.420 \%$ & $2.131 \%$ & $2.711 \%$ & $3.133 \%$ & $3.500 \%$ & $3.717 \%$ & $3.932 \%$ & $4.677 \%$ & $5.092 \%$ \\
\hline 7 & $1.204 \%$ & $1.756 \%$ & $2.091 \%$ & $2.415 \%$ & $2.503 \%$ & $2.611 \%$ & $3.231 \%$ & $10.726 \%$ & $9.931 \%$ & $5.963 \%$ & $4.451 \%$ \\
\hline 8 & $1.193 \%$ & $1.580 \%$ & $1.835 \%$ & $2.460 \%$ & $3.200 \%$ & $3.912 \%$ & $4.505 \%$ & $5.180 \%$ & $5.792 \%$ & $6.082 \%$ & $7.416 \%$ \\
\hline 9 & $0.856 \%$ & $1.152 \%$ & $1.552 \%$ & $2.175 \%$ & $2.674 \%$ & $3.242 \%$ & $3.883 \%$ & $4.571 \%$ & $5.332 \%$ & $6.318 \%$ & $7.306 \%$ \\
\hline $10^{*}$ & ---- & ---- & ---- & ---- & ---- & ---- & ---- & ---- & ---- & ---- & ---- \\
\hline 11 & $0.912 \%$ & $1.474 \%$ & $2.030 \%$ & $2.982 \%$ & $3.645 \%$ & $4.776 \%$ & $5.542 \%$ & $7.516 \%$ & $10.001 \%$ & $12.095 \%$ & $14.740 \%$ \\
\hline 12 & $1.351 \%$ & $2.445 \%$ & $2.667 \%$ & $3.699 \%$ & $3.573 \%$ & $4.259 \%$ & $5.132 \%$ & $6.184 \%$ & $7.582 \%$ & $6.591 \%$ & $10.042 \%$ \\
\hline 13 & $1.638 \%$ & $2.198 \%$ & $2.787 \%$ & $3.357 \%$ & $7.412 \%$ & $5.246 \%$ & $8.694 \%$ & $8.153 \%$ & $8.872 \%$ & $10.955 \%$ & $7.576 \%$ \\
\hline 14 & $0.742 \%$ & $1.128 \%$ & $1.301 \%$ & $1.722 \%$ & $2.089 \%$ & $2.390 \%$ & $2.584 \%$ & $2.863 \%$ & $3.335 \%$ & $3.803 \%$ & $4.292 \%$ \\
\hline 15 & $0.761 \%$ & $1.023 \%$ & $1.193 \%$ & $1.553 \%$ & $1.941 \%$ & $2.271 \%$ & $2.529 \%$ & $2.830 \%$ & $3.159 \%$ & $3.477 \%$ & $3.791 \%$ \\
\hline 16 & $0.701 \%$ & $0.966 \%$ & $1.340 \%$ & $2.077 \%$ & $2.559 \%$ & $2.858 \%$ & $3.046 \%$ & $3.209 \%$ & $3.633 \%$ & $4.481 \%$ & $4.656 \%$ \\
\hline 17 & $1.006 \%$ & $1.322 \%$ & $1.531 \%$ & $2.034 \%$ & $2.421 \%$ & $2.947 \%$ & $4.261 \%$ & $4.526 \%$ & $4.633 \%$ & $5.313 \%$ & $6.556 \%$ \\
\hline 18 & $1.424 \%$ & $2.385 \%$ & $3.043 \%$ & $3.364 \%$ & $3.677 \%$ & $4.555 \%$ & $6.027 \%$ & $5.803 \%$ & $6.048 \%$ & $7.670 \%$ & $9.080 \%$ \\
\hline 19 & $0.821 \%$ & $1.231 \%$ & $1.479 \%$ & $1.953 \%$ & $2.435 \%$ & $3.130 \%$ & $4.009 \%$ & $4.381 \%$ & $6.290 \%$ & $6.749 \%$ & $8.636 \%$ \\
\hline 20 & $1.363 \%$ & $2.152 \%$ & $2.503 \%$ & $2.697 \%$ & $3.195 \%$ & $3.815 \%$ & $5.318 \%$ & $7.416 \%$ & $9.050 \%$ & $11.253 \%$ & $15.716 \%$ \\
\hline 21 & $1.168 \%$ & $1.792 \%$ & $2.138 \%$ & $2.451 \%$ & $2.978 \%$ & $4.306 \%$ & $5.007 \%$ & $6.217 \%$ & $7.675 \%$ & $7.266 \%$ & $7.695 \%$ \\
\hline $22^{*}$ & ---- & ---- & ---- & ---- & ---- & ---- & ---- & ---- & ---- & ---- & ---- \\
\hline
\end{tabular}

\footnotetext{
*This ground motion was disqualified for reasons discussed in Appendix B.
} 
Table D.7: 9-Story drifts for load intensity only

\begin{tabular}{|c|c|c|c|c|c|c|c|c|c|c|c|}
\hline \multirow{2}{*}{$\begin{array}{l}\text { Ground } \\
\text { Motion }\end{array}$} & \multicolumn{11}{|l|}{ PSa (g) } \\
\hline & 0.1 & 0.15 & 0.2 & 0.3 & 0.4 & 0.5 & 0.6 & 0.7 & 0.8 & 0.9 & 1.0 \\
\hline 1 & $1.738 \%$ & $2.495 \%$ & $2.398 \%$ & $3.105 \%$ & $3.215 \%$ & $4.181 \%$ & $4.836 \%$ & $6.513 \%$ & $8.348 \%$ & $6.581 \%$ & $6.733 \%$ \\
\hline 2 & $0.847 \%$ & $1.095 \%$ & $1.383 \%$ & $2.218 \%$ & $3.032 \%$ & $3.416 \%$ & $3.514 \%$ & $3.456 \%$ & $3.968 \%$ & $4.563 \%$ & $4.926 \%$ \\
\hline 3 & $1.440 \%$ & $2.354 \%$ & $2.942 \%$ & $3.645 \%$ & $4.209 \%$ & $4.481 \%$ & $4.703 \%$ & $4.868 \%$ & $5.186 \%$ & $5.337 \%$ & $6.039 \%$ \\
\hline 4 & $1.049 \%$ & $1.543 \%$ & $1.770 \%$ & $2.002 \%$ & $2.636 \%$ & $3.039 \%$ & $3.230 \%$ & $3.808 \%$ & $4.315 \%$ & $4.697 \%$ & $5.224 \%$ \\
\hline 5 & $1.333 \%$ & $1.726 \%$ & $1.842 \%$ & $2.618 \%$ & $3.160 \%$ & $3.636 \%$ & $4.158 \%$ & $4.970 \%$ & $5.372 \%$ & $5.561 \%$ & $6.149 \%$ \\
\hline 6 & $0.787 \%$ & $1.087 \%$ & $1.402 \%$ & $2.142 \%$ & $2.643 \%$ & $3.156 \%$ & $3.544 \%$ & $3.733 \%$ & $4.026 \%$ & $4.556 \%$ & $5.159 \%$ \\
\hline 7 & $1.152 \%$ & $1.712 \%$ & $2.044 \%$ & $2.409 \%$ & $2.503 \%$ & $2.919 \%$ & $3.329 \%$ & $5.460 \%$ & $6.438 \%$ & $5.288 \%$ & $4.410 \%$ \\
\hline 8 & $1.161 \%$ & $1.531 \%$ & $1.776 \%$ & $2.423 \%$ & $3.143 \%$ & $3.918 \%$ & $4.535 \%$ & $5.126 \%$ & $5.771 \%$ & $6.205 \%$ & $6.753 \%$ \\
\hline 9 & $0.746 \%$ & $1.134 \%$ & $1.528 \%$ & $2.114 \%$ & $2.636 \%$ & $3.240 \%$ & $3.890 \%$ & $4.567 \%$ & $5.382 \%$ & $6.318 \%$ & $7.322 \%$ \\
\hline $10 *$ & ---- & ---- & ---- & ---- & ---- & ---- & ---- & ---- & ---- & ---- & ---- \\
\hline 11 & $0.970 \%$ & $1.460 \%$ & $2.010 \%$ & $2.963 \%$ & $3.651 \%$ & $4.645 \%$ & $5.485 \%$ & $7.422 \%$ & $9.839 \%$ & $12.139 \%$ & $14.796 \%$ \\
\hline 12 & $2.216 \%$ & $2.897 \%$ & $2.878 \%$ & $3.634 \%$ & $3.581 \%$ & $4.579 \%$ & $5.125 \%$ & $6.227 \%$ & $7.508 \%$ & $8.762 \%$ & $10.130 \%$ \\
\hline 13 & $1.652 \%$ & $2.184 \%$ & $2.799 \%$ & $3.564 \%$ & $4.854 \%$ & $5.189 \%$ & $7.144 \%$ & $8.593 \%$ & $7.829 \%$ & $9.183 \%$ & $7.880 \%$ \\
\hline 14 & $0.741 \%$ & $1.115 \%$ & $1.297 \%$ & $1.696 \%$ & $2.077 \%$ & $2.378 \%$ & $2.580 \%$ & $2.853 \%$ & $3.322 \%$ & $3.790 \%$ & $4.271 \%$ \\
\hline 15 & $0.715 \%$ & $0.974 \%$ & $1.167 \%$ & $1.561 \%$ & $1.940 \%$ & $2.239 \%$ & $2.530 \%$ & $2.844 \%$ & $3.152 \%$ & $3.463 \%$ & $3.781 \%$ \\
\hline 16 & $0.663 \%$ & $0.960 \%$ & $1.273 \%$ & $2.027 \%$ & $2.478 \%$ & $2.760 \%$ & $2.949 \%$ & $3.357 \%$ & $3.600 \%$ & $4.346 \%$ & $4.531 \%$ \\
\hline 17 & $1.015 \%$ & $1.261 \%$ & $1.507 \%$ & $1.973 \%$ & $2.417 \%$ & $2.926 \%$ & $3.418 \%$ & $3.920 \%$ & $4.391 \%$ & $4.959 \%$ & $5.667 \%$ \\
\hline 18 & $1.430 \%$ & $2.354 \%$ & $3.014 \%$ & $3.344 \%$ & $3.680 \%$ & $4.645 \%$ & $5.259 \%$ & $5.722 \%$ & $6.073 \%$ & $7.409 \%$ & $8.862 \%$ \\
\hline 19 & $0.811 \%$ & $1.216 \%$ & $1.484 \%$ & $1.941 \%$ & $2.469 \%$ & $3.120 \%$ & $4.012 \%$ & $4.580 \%$ & $6.286 \%$ & $7.086 \%$ & $8.460 \%$ \\
\hline 20 & $1.342 \%$ & $2.068 \%$ & $2.462 \%$ & $2.716 \%$ & $3.173 \%$ & $3.778 \%$ & $5.188 \%$ & $6.855 \%$ & $9.005 \%$ & $11.342 \%$ & $14.530 \%$ \\
\hline 21 & $1.132 \%$ & $1.735 \%$ & $2.138 \%$ & $2.493 \%$ & $2.953 \%$ & $3.453 \%$ & $4.485 \%$ & $5.932 \%$ & $6.781 \%$ & $7.099 \%$ & $7.618 \%$ \\
\hline $22^{*}$ & ---- & ---- & ---- & ---- & ---- & ---- & ---- & ---- & ---- & ---- & ---- \\
\hline
\end{tabular}

\footnotetext{
*This ground motion was disqualified for reasons discussed in Appendix B.
} 
Table D.8: 9-Story drifts for mean load

\begin{tabular}{|c|c|c|c|c|c|c|c|c|c|c|c|}
\hline $\begin{array}{l}\text { Ground } \\
\text { Motion }\end{array}$ & $\begin{array}{l}\text { PSa (g) } \\
0.1 \\
\end{array}$ & 0.15 & 0.2 & 0.3 & 0.4 & 0.5 & 0.6 & 0.7 & 0.8 & 0.9 & 1.0 \\
\hline 1 & $1.552 \%$ & $2.186 \%$ & $2.500 \%$ & $2.912 \%$ & $3.533 \%$ & $4.141 \%$ & $5.114 \%$ & $5.940 \%$ & $6.437 \%$ & $7.128 \%$ & $7.685 \%$ \\
\hline 2 & $0.893 \%$ & $1.126 \%$ & $1.386 \%$ & $2.259 \%$ & $3.096 \%$ & $3.472 \%$ & $3.575 \%$ & $3.647 \%$ & $3.948 \%$ & $4.456 \%$ & $4.958 \%$ \\
\hline 3 & $1.436 \%$ & $2.387 \%$ & $2.979 \%$ & $3.681 \%$ & $4.205 \%$ & $4.512 \%$ & $4.689 \%$ & $4.909 \%$ & $5.138 \%$ & $5.300 \%$ & $5.756 \%$ \\
\hline 4 & $1.039 \%$ & $1.616 \%$ & $1.876 \%$ & $1.891 \%$ & $2.645 \%$ & $3.085 \%$ & $3.291 \%$ & $3.783 \%$ & $4.365 \%$ & $4.708 \%$ & $5.154 \%$ \\
\hline 5 & $0.992 \%$ & $1.503 \%$ & $1.967 \%$ & $2.705 \%$ & $3.268 \%$ & $3.653 \%$ & $4.244 \%$ & $4.862 \%$ & $5.289 \%$ & $5.452 \%$ & $5.534 \%$ \\
\hline 6 & $0.786 \%$ & $1.063 \%$ & $1.428 \%$ & $2.159 \%$ & $2.728 \%$ & $3.119 \%$ & $3.535 \%$ & $3.717 \%$ & $3.860 \%$ & $4.325 \%$ & $4.743 \%$ \\
\hline 7 & $1.214 \%$ & $1.767 \%$ & $2.109 \%$ & $2.427 \%$ & $2.483 \%$ & $2.558 \%$ & $3.203 \%$ & $3.806 \%$ & $4.619 \%$ & $5.409 \%$ & $6.204 \%$ \\
\hline 8 & $1.198 \%$ & $1.585 \%$ & $1.841 \%$ & $2.463 \%$ & $3.203 \%$ & $3.910 \%$ & $4.558 \%$ & $5.108 \%$ & $5.738 \%$ & $6.366 \%$ & $6.887 \%$ \\
\hline 9 & $0.756 \%$ & $1.154 \%$ & $1.554 \%$ & $2.167 \%$ & $2.659 \%$ & $3.237 \%$ & $3.871 \%$ & $4.577 \%$ & $5.420 \%$ & $6.336 \%$ & $7.308 \%$ \\
\hline $10^{*}$ & ---- & ---- & ---- & ---- & ---- & ---- & ---- & ---- & ---- & ---- & ---- \\
\hline 11 & $0.887 \%$ & $1.470 \%$ & $2.030 \%$ & $2.985 \%$ & $3.643 \%$ & $4.783 \%$ & $5.554 \%$ & $7.526 \%$ & $9.688 \%$ & $12.175 \%$ & $15.021 \%$ \\
\hline 12 & $1.312 \%$ & $2.309 \%$ & $2.641 \%$ & $3.624 \%$ & $4.384 \%$ & $4.376 \%$ & $5.195 \%$ & $6.163 \%$ & $7.247 \%$ & $8.606 \%$ & $9.914 \%$ \\
\hline 13 & $1.637 \%$ & $2.206 \%$ & $2.799 \%$ & $3.415 \%$ & $4.159 \%$ & $5.187 \%$ & $6.854 \%$ & $7.451 \%$ & $8.118 \%$ & $8.300 \%$ & $8.657 \%$ \\
\hline 14 & $0.735 \%$ & $1.130 \%$ & $1.307 \%$ & $1.723 \%$ & $2.093 \%$ & $2.393 \%$ & $2.589 \%$ & $2.863 \%$ & $3.334 \%$ & $3.805 \%$ & $4.291 \%$ \\
\hline 15 & $0.746 \%$ & $0.960 \%$ & $1.199 \%$ & $1.553 \%$ & $1.942 \%$ & $2.278 \%$ & $2.529 \%$ & $2.820 \%$ & $3.153 \%$ & $3.479 \%$ & $3.785 \%$ \\
\hline 16 & $0.677 \%$ & $0.969 \%$ & $1.287 \%$ & $2.081 \%$ & $2.567 \%$ & $2.869 \%$ & $3.060 \%$ & $3.218 \%$ & $3.641 \%$ & $4.154 \%$ & $4.651 \%$ \\
\hline 17 & $1.032 \%$ & $1.233 \%$ & $1.535 \%$ & $2.036 \%$ & $2.426 \%$ & $2.951 \%$ & $3.428 \%$ & $3.911 \%$ & $4.377 \%$ & $4.876 \%$ & $5.301 \%$ \\
\hline 18 & $1.425 \%$ & $2.387 \%$ & $3.045 \%$ & $3.362 \%$ & $3.676 \%$ & $4.518 \%$ & $5.303 \%$ & $5.857 \%$ & $6.161 \%$ & $7.395 \%$ & $8.914 \%$ \\
\hline 19 & $0.821 \%$ & $1.232 \%$ & $1.471 \%$ & $1.955 \%$ & $2.429 \%$ & $3.129 \%$ & $4.003 \%$ & $5.127 \%$ & $6.221 \%$ & $7.344 \%$ & $8.313 \%$ \\
\hline 20 & $1.354 \%$ & $2.151 \%$ & $2.518 \%$ & $2.717 \%$ & $3.300 \%$ & $3.947 \%$ & $5.243 \%$ & $6.834 \%$ & $8.960 \%$ & $11.289 \%$ & $13.829 \%$ \\
\hline 21 & $1.180 \%$ & $1.805 \%$ & $2.100 \%$ & $2.464 \%$ & $2.958 \%$ & $3.534 \%$ & $4.592 \%$ & $5.512 \%$ & $6.325 \%$ & $6.935 \%$ & $7.531 \%$ \\
\hline $22 *$ & ---- & ---- & ---- & ---- & ---- & ---- & ---- & ---- & ---- & ---- & ---- \\
\hline
\end{tabular}

*This ground motion was disqualified for reasons discussed in Appendix B. 
Table D.9: 20-Story drifts for load eccentricity and intensity

\begin{tabular}{|c|c|c|c|c|c|c|c|}
\hline \multirow{2}{*}{$\begin{array}{l}\text { Ground } \\
\text { Motion }\end{array}$} & \multicolumn{7}{|l|}{ PSa (g) } \\
\hline & 0.1 & 0.15 & 0.2 & 0.3 & 0.4 & 0.5 & 0.6 \\
\hline 1 & $1.199 \%$ & $1.426 \%$ & $2.282 \%$ & $3.641 \%$ & $4.709 \%$ & $5.601 \%$ & $6.282 \%$ \\
\hline 2 & $0.872 \%$ & $1.280 \%$ & $1.731 \%$ & $3.034 \%$ & $3.954 \%$ & $4.730 \%$ & $5.531 \%$ \\
\hline 3 & $1.016 \%$ & $1.255 \%$ & $1.318 \%$ & $2.108 \%$ & $2.668 \%$ & $3.361 \%$ & $4.022 \%$ \\
\hline 4 & $1.104 \%$ & $1.260 \%$ & $1.786 \%$ & $3.072 \%$ & $4.024 \%$ & $4.836 \%$ & $5.629 \%$ \\
\hline 5 & $0.658 \%$ & $0.909 \%$ & $1.169 \%$ & $1.754 \%$ & $2.172 \%$ & $2.627 \%$ & $3.077 \%$ \\
\hline 6 & $0.700 \%$ & $0.938 \%$ & $1.113 \%$ & $1.683 \%$ & $2.383 \%$ & $2.916 \%$ & $3.368 \%$ \\
\hline 7 & $1.075 \%$ & $1.300 \%$ & $1.614 \%$ & $2.663 \%$ & $3.697 \%$ & $4.794 \%$ & $5.640 \%$ \\
\hline 8 & $0.881 \%$ & $1.119 \%$ & $1.250 \%$ & $2.081 \%$ & $2.734 \%$ & $3.476 \%$ & $4.225 \%$ \\
\hline 9 & $1.209 \%$ & $2.036 \%$ & $2.630 \%$ & $3.667 \%$ & $5.169 \%$ & $7.033 \%$ & $8.743 \%$ \\
\hline $10 *$ & ---- & ---- & ---- & ---- & ---- & ---- & ---- \\
\hline 11 & $0.914 \%$ & $1.586 \%$ & $1.974 \%$ & $4.285 \%$ & $7.459 \%$ & $10.784 \%$ & $14.152 \%$ \\
\hline $12 *$ & ---- & ---- & ---- & ---- & ---- & ---- & ---- \\
\hline 13 & $1.366 \%$ & $1.731 \%$ & $2.767 \%$ & $4.343 \%$ & $5.081 \%$ & $6.032 \%$ & $6.911 \%$ \\
\hline 14 & $0.804 \%$ & $1.340 \%$ & $1.425 \%$ & $1.912 \%$ & $2.778 \%$ & $3.586 \%$ & $4.317 \%$ \\
\hline 15 & $0.640 \%$ & $0.971 \%$ & $1.260 \%$ & $1.749 \%$ & $2.717 \%$ & $2.864 \%$ & $3.181 \%$ \\
\hline 16 & $0.830 \%$ & $1.219 \%$ & $1.261 \%$ & $1.787 \%$ & $2.656 \%$ & $3.555 \%$ & $4.513 \%$ \\
\hline 17 & $0.856 \%$ & $1.324 \%$ & $1.495 \%$ & $2.693 \%$ & $3.858 \%$ & $4.895 \%$ & $5.724 \%$ \\
\hline $18^{*}$ & ---- & ---- & ---- & ---- & ---- & ---- & ---- \\
\hline $19 *$ & ---- & ---- & ---- & ---- & ---- & ---- & ---- \\
\hline $20 *$ & ---- & ---- & ---- & ---- & ---- & ---- & ---- \\
\hline 21 & $0.937 \%$ & $1.259 \%$ & $1.568 \%$ & $2.529 \%$ & $3.145 \%$ & $1.463 \%$ & $4.476 \%$ \\
\hline 22 & $1.547 \%$ & $2.384 \%$ & $3.286 \%$ & $5.285 \%$ & $7.334 \%$ & $8.931 \%$ & $12.261 \%$ \\
\hline
\end{tabular}

*This ground motion was disqualified for reasons discussed in Appendix B. 
Table D.10: 20-Story drifts for load eccentricity only

\begin{tabular}{|c|c|c|c|c|c|c|c|}
\hline Ground & PSa (g) & & & & & & \\
\hline Motion & 0.1 & 0.15 & 0.2 & 0.3 & 0.4 & 0.5 & 0.6 \\
\hline 1 & $1.200 \%$ & $1.443 \%$ & $2.263 \%$ & $3.634 \%$ & $4.708 \%$ & $5.603 \%$ & $6.263 \%$ \\
\hline 2 & $0.875 \%$ & $1.273 \%$ & $1.727 \%$ & $3.037 \%$ & $3.959 \%$ & $4.744 \%$ & $5.516 \%$ \\
\hline 3 & $1.023 \%$ & $1.268 \%$ & $1.320 \%$ & $2.106 \%$ & $2.681 \%$ & $3.352 \%$ & $4.047 \%$ \\
\hline 4 & $1.108 \%$ & $1.264 \%$ & $1.802 \%$ & $3.084 \%$ & $4.022 \%$ & $4.842 \%$ & $5.631 \%$ \\
\hline 5 & $0.637 \%$ & $0.917 \%$ & $1.174 \%$ & $1.753 \%$ & $2.168 \%$ & $2.585 \%$ & $3.114 \%$ \\
\hline 6 & $0.705 \%$ & 0.937\% & 1.099\% & $1.678 \%$ & $2.368 \%$ & $2.903 \%$ & $3.364 \%$ \\
\hline 7 & $1.100 \%$ & $1.299 \%$ & $1.612 \%$ & $2.656 \%$ & $3.711 \%$ & $4.789 \%$ & $5.648 \%$ \\
\hline 8 & $0.892 \%$ & $1.116 \%$ & $1.228 \%$ & $2.072 \%$ & $2.740 \%$ & $3.399 \%$ & $4.216 \%$ \\
\hline 9 & $1.209 \%$ & $2.039 \%$ & $2.621 \%$ & $3.667 \%$ & $5.172 \%$ & $7.028 \%$ & $8.709 \%$ \\
\hline $10 *$ & ---- & ---- & ---- & ---- & ---- & ---- & ---- \\
\hline 11 & $0.918 \%$ & $1.589 \%$ & $1.977 \%$ & $4.286 \%$ & $7.472 \%$ & $10.802 \%$ & $14.157 \%$ \\
\hline $12 *$ & ---- & --- & ---- & ---- & ---- & ---- & ---- \\
\hline 13 & $1.258 \%$ & $1.715 \%$ & $2.763 \%$ & $4.347 \%$ & $5.067 \%$ & $6.044 \%$ & $6.911 \%$ \\
\hline 14 & $0.791 \%$ & $1.334 \%$ & $1.433 \%$ & $1.914 \%$ & $2.771 \%$ & $3.589 \%$ & $4.324 \%$ \\
\hline 15 & $0.640 \%$ & $0.980 \%$ & $1.266 \%$ & $1.740 \%$ & $2.724 \%$ & $2.866 \%$ & $3.206 \%$ \\
\hline 16 & $0.832 \%$ & $1.223 \%$ & $1.259 \%$ & $1.759 \%$ & $2.639 \%$ & $3.559 \%$ & $4.521 \%$ \\
\hline 17 & $0.847 \%$ & $1.327 \%$ & $1.451 \%$ & $2.701 \%$ & $3.877 \%$ & $4.889 \%$ & $5.738 \%$ \\
\hline $18 *$ & ---- & ---- & ---- & ---- & ---- & ---- & ---- \\
\hline $19 *$ & ---- & ---- & ---- & ---- & ---- & ---- & ---- \\
\hline $20 *$ & ---- & ---- & ---- & ---- & ---- & ---- & ---- \\
\hline 21 & $0.959 \%$ & $1.297 \%$ & $1.557 \%$ & $2.496 \%$ & $3.137 \%$ & $3.819 \%$ & $4.371 \%$ \\
\hline 22 & $1.569 \%$ & $2.400 \%$ & $3.297 \%$ & $5.209 \%$ & $7.376 \%$ & $9.137 \%$ & $11.148 \%$ \\
\hline
\end{tabular}

*This ground motion was disqualified for reasons discussed in Appendix B. 
Table D.11: 20-Story drifts for load intensity only

\begin{tabular}{|c|c|c|c|c|c|c|c|}
\hline Ground & PSa (g) & & & & & & \\
\hline Motion & 0.1 & 0.15 & 0.2 & 0.3 & 0.4 & 0.5 & 0.6 \\
\hline 1 & $1.200 \%$ & $1.431 \%$ & $2.279 \%$ & $3.624 \%$ & $4.691 \%$ & $5.599 \%$ & $6.271 \%$ \\
\hline 2 & $0.838 \%$ & $1.267 \%$ & $1.731 \%$ & $3.032 \%$ & $3.954 \%$ & $4.730 \%$ & $5.503 \%$ \\
\hline 3 & $1.016 \%$ & $1.252 \%$ & $1.320 \%$ & $2.099 \%$ & $2.675 \%$ & $3.360 \%$ & $4.040 \%$ \\
\hline 4 & $1.107 \%$ & $1.260 \%$ & $1.793 \%$ & $3.075 \%$ & $4.020 \%$ & $4.847 \%$ & $5.623 \%$ \\
\hline 5 & $0.651 \%$ & $0.909 \%$ & $1.131 \%$ & $1.759 \%$ & $2.179 \%$ & $2.615 \%$ & $3.056 \%$ \\
\hline 6 & $0.700 \%$ & $0.938 \%$ & $1.110 \%$ & $1.686 \%$ & $2.384 \%$ & $2.894 \%$ & $3.362 \%$ \\
\hline 7 & $1.089 \%$ & $1.295 \%$ & $1.615 \%$ & $2.652 \%$ & $3.705 \%$ & $4.789 \%$ & $5.652 \%$ \\
\hline 8 & $0.868 \%$ & $1.045 \%$ & $1.237 \%$ & $2.059 \%$ & $2.744 \%$ & $3.497 \%$ & $4.209 \%$ \\
\hline 9 & $1.210 \%$ & $2.053 \%$ & $2.628 \%$ & $3.673 \%$ & $5.171 \%$ & $7.030 \%$ & $8.736 \%$ \\
\hline $10 *$ & ---- & ---- & ---- & ---- & ---- & ---- & ---- \\
\hline 11 & $0.912 \%$ & $1.513 \%$ & $1.980 \%$ & $4.276 \%$ & $7.471 \%$ & $10.816 \%$ & $14.146 \%$ \\
\hline $12 *$ & ---- & ---- & ---- & ---- & ---- & ---- & ---- \\
\hline 13 & $1.320 \%$ & $1.683 \%$ & $2.759 \%$ & $4.343 \%$ & $5.085 \%$ & $6.017 \%$ & $6.908 \%$ \\
\hline 14 & $0.787 \%$ & $1.274 \%$ & $1.370 \%$ & $1.912 \%$ & $2.769 \%$ & $3.583 \%$ & $4.327 \%$ \\
\hline 15 & $0.640 \%$ & $0.972 \%$ & $1.261 \%$ & $1.754 \%$ & $2.711 \%$ & $2.863 \%$ & $3.204 \%$ \\
\hline 16 & $0.831 \%$ & $1.219 \%$ & $1.260 \%$ & $1.784 \%$ & $2.649 \%$ & $3.555 \%$ & $4.500 \%$ \\
\hline 17 & $0.837 \%$ & $1.325 \%$ & $1.496 \%$ & $2.695 \%$ & $3.872 \%$ & $4.891 \%$ & $5.741 \%$ \\
\hline $18^{*}$ & ---- & ---- & ---- & ---- & ---- & ---- & ---- \\
\hline $19 *$ & ---- & ---- & ---- & ---- & ---- & ---- & ---- \\
\hline $20 *$ & ---- & ---- & ---- & ---- & ---- & ---- & ---- \\
\hline 21 & $0.940 \%$ & $1.261 \%$ & $1.569 \%$ & $2.520 \%$ & $3.157 \%$ & $1.458 \%$ & $4.482 \%$ \\
\hline 22 & $1.510 \%$ & $2.398 \%$ & $3.293 \%$ & $5.288 \%$ & $7.348 \%$ & $9.016 \%$ & $12.310 \%$ \\
\hline
\end{tabular}


Table D.12: 20-Story drifts for mean load

\begin{tabular}{|c|c|c|c|c|c|c|c|}
\hline \multirow{2}{*}{$\begin{array}{l}\text { Ground } \\
\text { Motion }\end{array}$} & \multicolumn{7}{|l|}{ PSa (g) } \\
\hline & 0.1 & 0.15 & 0.2 & 0.3 & 0.4 & 0.5 & 0.6 \\
\hline 1 & $1.201 \%$ & $1.448 \%$ & $2.259 \%$ & $3.617 \%$ & $4.690 \%$ & $5.601 \%$ & $6.252 \%$ \\
\hline 2 & $0.843 \%$ & $1.283 \%$ & $1.727 \%$ & $3.034 \%$ & $3.960 \%$ & $4.744 \%$ & $5.488 \%$ \\
\hline 3 & $1.022 \%$ & $1.265 \%$ & $1.322 \%$ & $2.098 \%$ & $2.688 \%$ & $3.352 \%$ & $4.066 \%$ \\
\hline 4 & $1.112 \%$ & $1.264 \%$ & $1.809 \%$ & $3.087 \%$ & $4.018 \%$ & $4.853 \%$ & $5.624 \%$ \\
\hline 5 & $0.630 \%$ & $0.918 \%$ & $1.136 \%$ & $1.758 \%$ & $2.174 \%$ & $2.574 \%$ & $3.093 \%$ \\
\hline 6 & $0.705 \%$ & $0.937 \%$ & $1.096 \%$ & $1.681 \%$ & $2.369 \%$ & $2.881 \%$ & $3.358 \%$ \\
\hline 7 & $1.114 \%$ & $1.294 \%$ & $1.612 \%$ & $2.645 \%$ & $3.719 \%$ & $4.785 \%$ & $5.659 \%$ \\
\hline 8 & $0.879 \%$ & $1.041 \%$ & $1.162 \%$ & $2.050 \%$ & $2.750 \%$ & $3.419 \%$ & $4.200 \%$ \\
\hline 9 & $1.209 \%$ & $2.056 \%$ & $2.618 \%$ & $3.673 \%$ & $5.174 \%$ & $7.026 \%$ & $8.703 \%$ \\
\hline $10 *$ & ---- & ---- & ---- & ---- & ---- & ---- & ---- \\
\hline 11 & $0.916 \%$ & $1.515 \%$ & $1.982 \%$ & $4.277 \%$ & $7.484 \%$ & $10.834 \%$ & $14.151 \%$ \\
\hline $12 *$ & ---- & ---- & ---- & ---- & ---- & ---- & ---- \\
\hline 13 & $1.257 \%$ & $1.667 \%$ & $2.754 \%$ & $4.347 \%$ & $5.071 \%$ & $6.028 \%$ & $6.908 \%$ \\
\hline 14 & $0.783 \%$ & $1.268 \%$ & $1.379 \%$ & $1.913 \%$ & $2.762 \%$ & $3.587 \%$ & $4.334 \%$ \\
\hline 15 & $0.640 \%$ & $0.981 \%$ & $1.267 \%$ & $1.744 \%$ & $2.718 \%$ & $2.866 \%$ & $3.230 \%$ \\
\hline 16 & $0.833 \%$ & $1.224 \%$ & $1.263 \%$ & $1.756 \%$ & $2.632 \%$ & $3.560 \%$ & $4.508 \%$ \\
\hline 17 & $0.828 \%$ & $1.327 \%$ & $1.451 \%$ & $2.703 \%$ & $3.891 \%$ & $4.885 \%$ & $5.754 \%$ \\
\hline $18^{*}$ & ---- & ---- & ---- & ---- & ---- & ---- & ---- \\
\hline $19 *$ & ---- & ---- & ---- & ---- & ---- & ---- & ---- \\
\hline $20 *$ & ---- & ---- & ---- & ---- & ---- & ---- & ---- \\
\hline 21 & $0.962 \%$ & $1.299 \%$ & $1.558 \%$ & $2.488 \%$ & $3.149 \%$ & $3.806 \%$ & $4.376 \%$ \\
\hline 22 & $1.531 \%$ & $2.414 \%$ & $3.304 \%$ & $5.212 \%$ & $7.390 \%$ & $9.224 \%$ & $11.192 \%$ \\
\hline
\end{tabular}




\begin{tabular}{|c|c|c|c|c|c|c|}
\hline \multirow[b]{2}{*}{$\begin{array}{l}\text { Ground } \\
\text { Motion }\end{array}$} & \multicolumn{2}{|l|}{ 3-Story } & \multicolumn{2}{|l|}{ 9-Story } & \multicolumn{2}{|l|}{ 20-Story } \\
\hline & $\begin{array}{l}\text { DBE } \\
0.825 \\
\text { (g) }\end{array}$ & $\begin{array}{l}\text { MCE } \\
1.244 \\
\text { (g) }\end{array}$ & $\begin{array}{l}\text { DBE } \\
0.365 \\
\text { (g) }\end{array}$ & $\begin{array}{l}\text { MCE } \\
0.547 \\
\text { (g) }\end{array}$ & $\begin{array}{l}\text { DBE } \\
0.240 \\
\text { (g) }\end{array}$ & $\begin{array}{l}\text { MCE } \\
0.316 \\
\text { (g) }\end{array}$ \\
\hline 1 & $2.453 \%$ & $3.638 \%$ & $3.824 \%$ & $5.597 \%$ & $4.230 \%$ & $5.371 \%$ \\
\hline 2 & $2.740 \%$ & $3.625 \%$ & $2.655 \%$ & $3.390 \%$ & $3.613 \%$ & $4.499 \%$ \\
\hline 3 & $2.641 \%$ & $3.182 \%$ & $4.248 \%$ & $4.681 \%$ & $2.229 \%$ & $2.877 \%$ \\
\hline 4 & $3.508 \%$ & $4.793 \%$ & $2.507 \%$ & $3.338 \%$ & $3.203 \%$ & $4.089 \%$ \\
\hline 5 & $3.224 \%$ & $5.033 \%$ & $3.262 \%$ & $4.236 \%$ & $2.147 \%$ & $2.634 \%$ \\
\hline 6 & $4.294 \%$ & $7.617 \%$ & $2.592 \%$ & $4.136 \%$ & $1.848 \%$ & $2.443 \%$ \\
\hline 7 & $4.180 \%$ & $5.469 \%$ & $2.280 \%$ & $3.099 \%$ & $2.982 \%$ & $4.235 \%$ \\
\hline 8 & $3.374 \%$ & $5.962 \%$ & $2.938 \%$ & $4.205 \%$ & $2.629 \%$ & $3.366 \%$ \\
\hline 9 & $3.618 \%$ & $5.511 \%$ & $2.476 \%$ & $3.618 \%$ & $2.597 \%$ & $3.306 \%$ \\
\hline $10 *$ & ---- & ---- & ---- & ---- & ---- & ---- \\
\hline 11 & $2.294 \%$ & $5.215 \%$ & $3.695 \%$ & $5.552 \%$ & $3.489 \%$ & $6.131 \%$ \\
\hline $12 *$ & $5.624 \%$ & $7.179 \%$ & $4.937 \%$ & $4.624 \%$ & ---- & ---- \\
\hline 13 & $3.226 \%$ & $4.750 \%$ & $4.618 \%$ & $6.577 \%$ & $4.649 \%$ & $5.431 \%$ \\
\hline 14 & $3.929 \%$ & $6.617 \%$ & $1.966 \%$ & $2.693 \%$ & $2.193 \%$ & $3.091 \%$ \\
\hline 15 & $2.452 \%$ & $3.602 \%$ & $1.787 \%$ & $2.393 \%$ & $2.545 \%$ & $2.808 \%$ \\
\hline 16 & $5.147 \%$ & $6.781 \%$ & $2.463 \%$ & $3.031 \%$ & $2.124 \%$ & $3.105 \%$ \\
\hline 17 & $2.878 \%$ & $4.818 \%$ & $2.387 \%$ & $3.215 \%$ & $2.802 \%$ & $3.971 \%$ \\
\hline $18 *$ & $3.463 \%$ & $4.469 \%$ & $4.202 \%$ & $5.821 \%$ & ---- & ---- \\
\hline $19 *$ & $2.894 \%$ & $4.082 \%$ & $2.486 \%$ & $3.937 \%$ & ---- & ---- \\
\hline $20 *$ & $2.908 \%$ & $4.726 \%$ & $3.354 \%$ & $4.753 \%$ & ---- & ---- \\
\hline 21 & $2.768 \%$ & $4.432 \%$ & $3.032 \%$ & $4.414 \%$ & $1.894 \%$ & $2.512 \%$ \\
\hline $22 *$ & $3.553 \%$ & $5.303 \%$ & ---- & ---- & $3.666 \%$ & $4.964 \%$ \\
\hline
\end{tabular}




\section{APPENDIX E: Fragility Curves}

All of the figures in Appendix E are of fragility curves for the three models. Starting with the three-story curves there are first smooth/regression and rough/non-regression curves are shown independently and then compared together. Next comparisons are shown between the smooth/regression curves and the FEMA 350 results. Finally the original FEMA 350 results are shown alone. This same sequence of fragility curves is repeated for the nine-story and twentystory models. Within each of the three different types of fragility curves produced from the different analysis procedures there are comparisons for the various loading scenarios. These different loading scenarios are abbreviated as such:

Ecc: refers to only the consideration of eccentric loading.

LI: refers to only the consideration of load intensity.

Ecc \& LI: refers to the consideration of both load eccentricity and intensity. Mean: refers to the consideration of neither load eccentricity or intensity. 


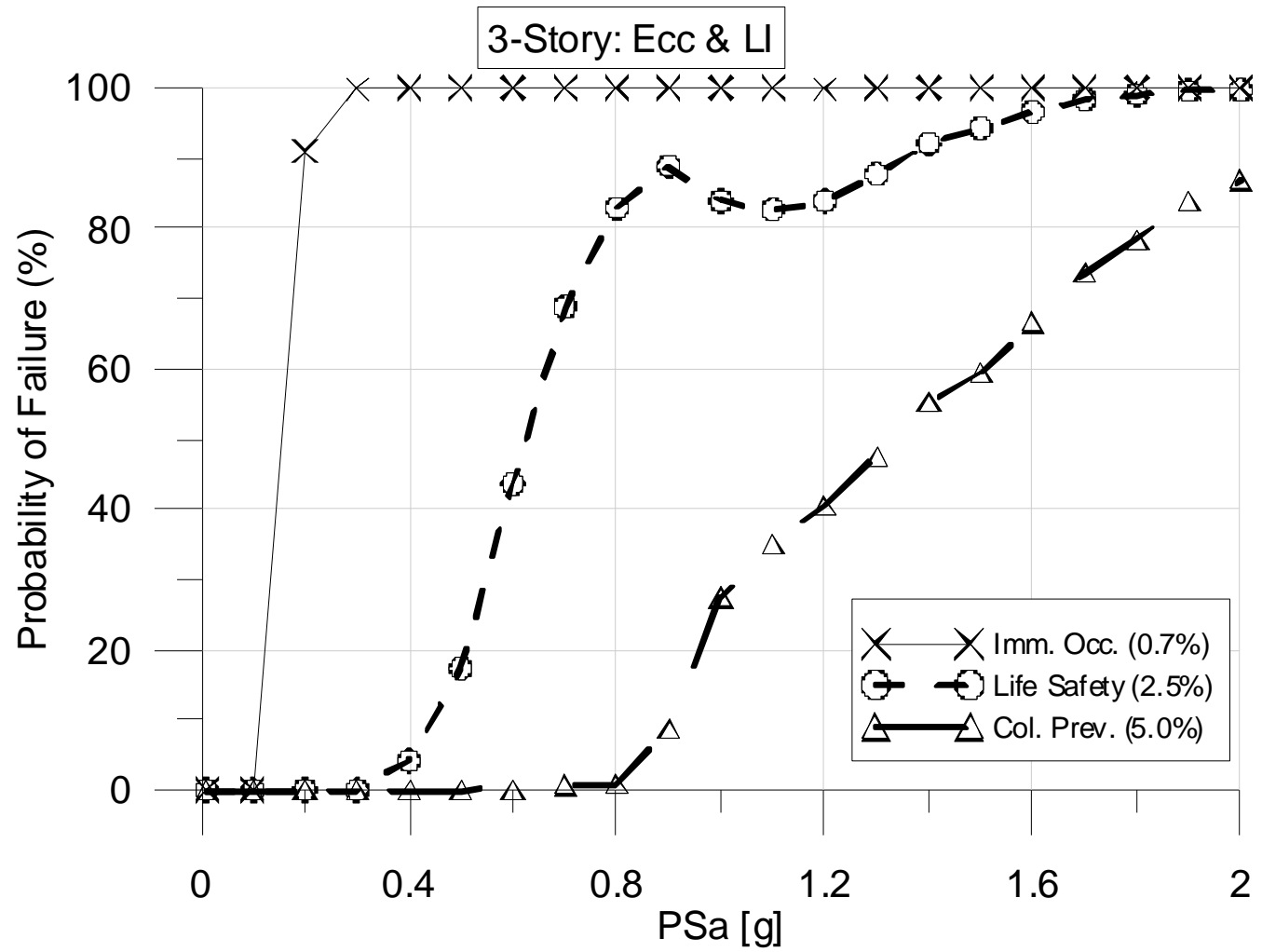

Figure E.1: Non-regression fragility curves for load eccentricity and intensity, 3-story

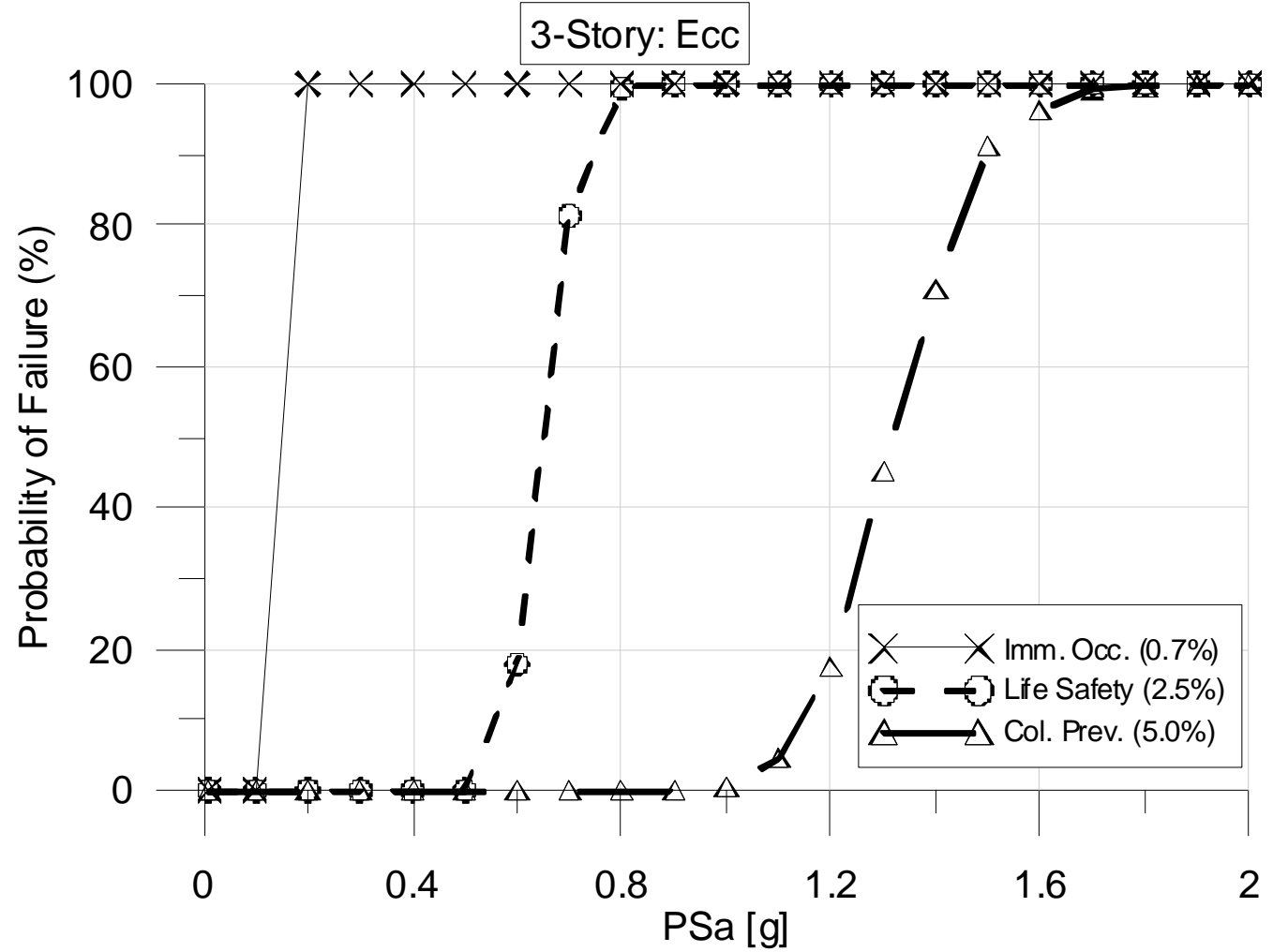

Figure E.2: Non-regression fragility curves for load eccentricity, 3-story 


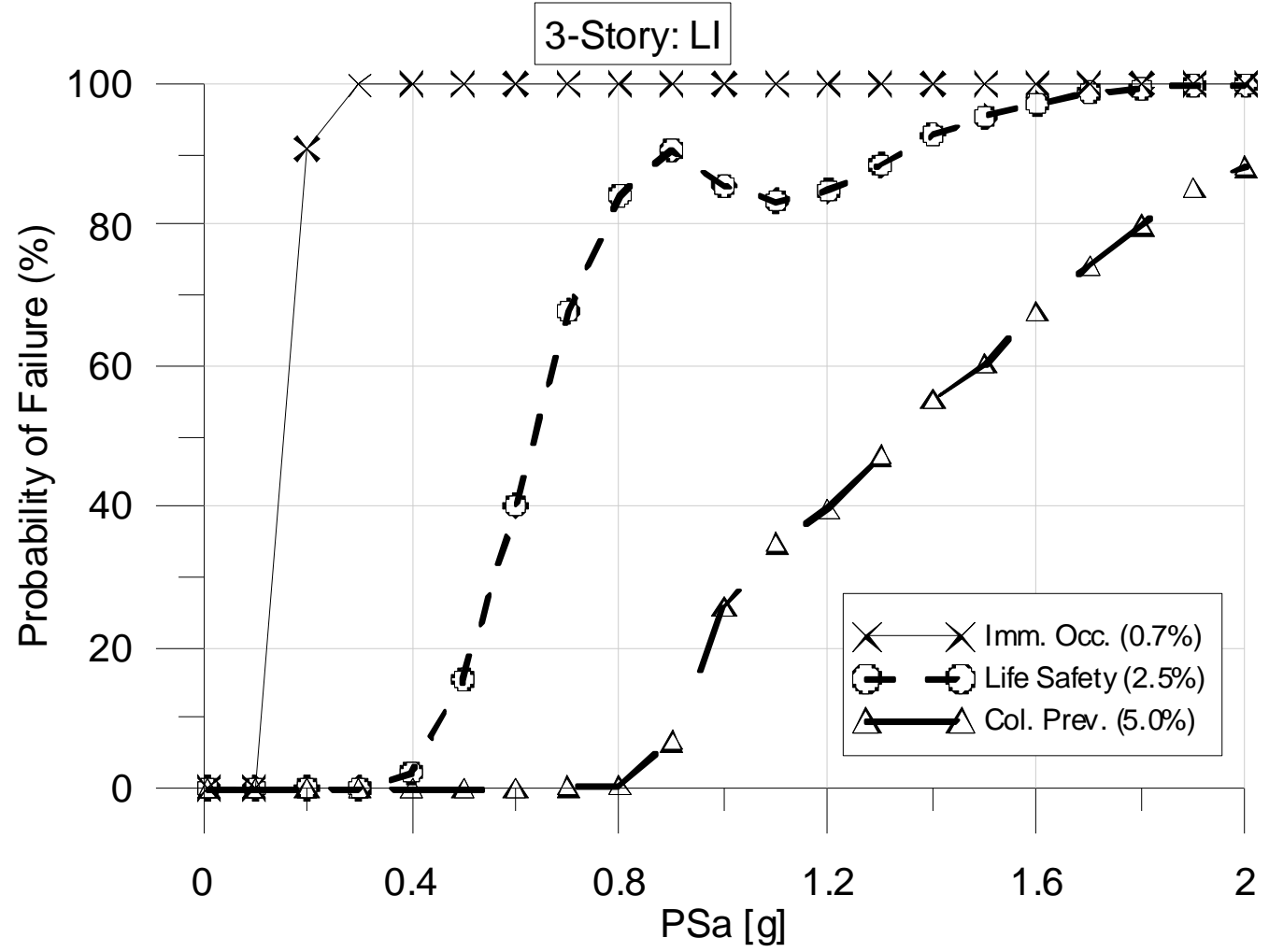

Figure E.3: Non-regression fragility curves for load intensity, 3-story

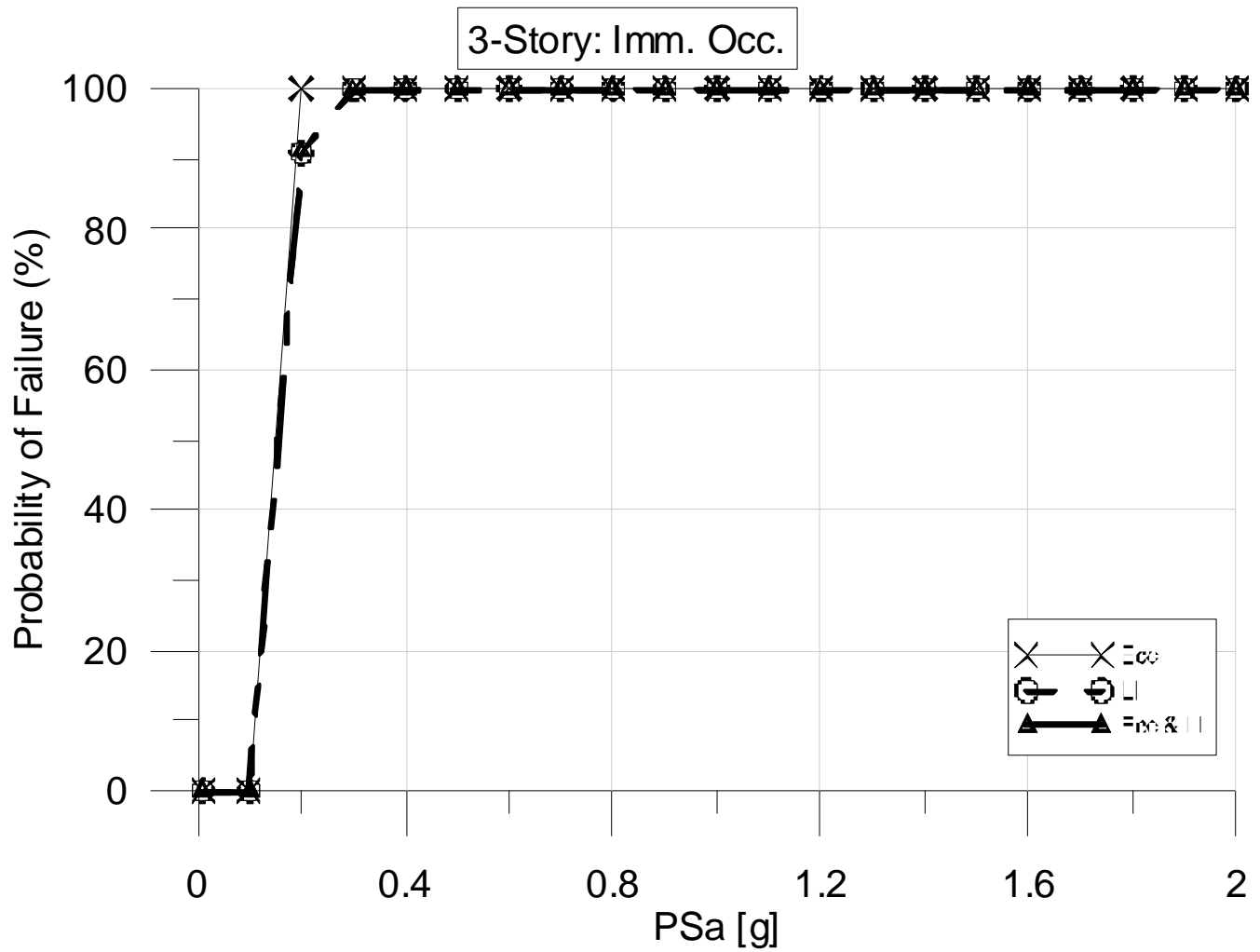

Figure E.4: Non-regression fragility curves immediate occupancy comparison, 3-story 


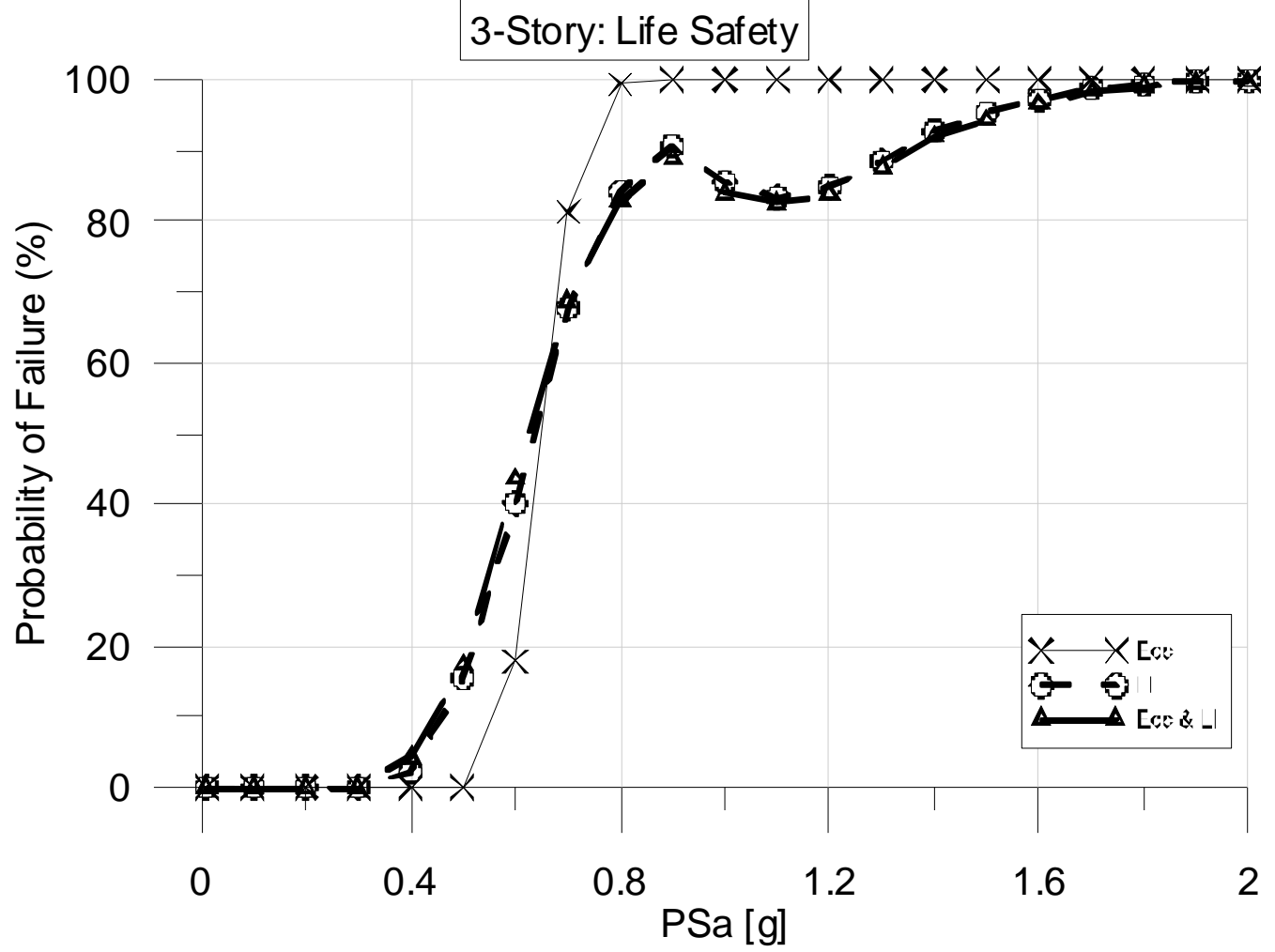

Figure E.5: Non-regression fragility curves life safety comparison, 3-story

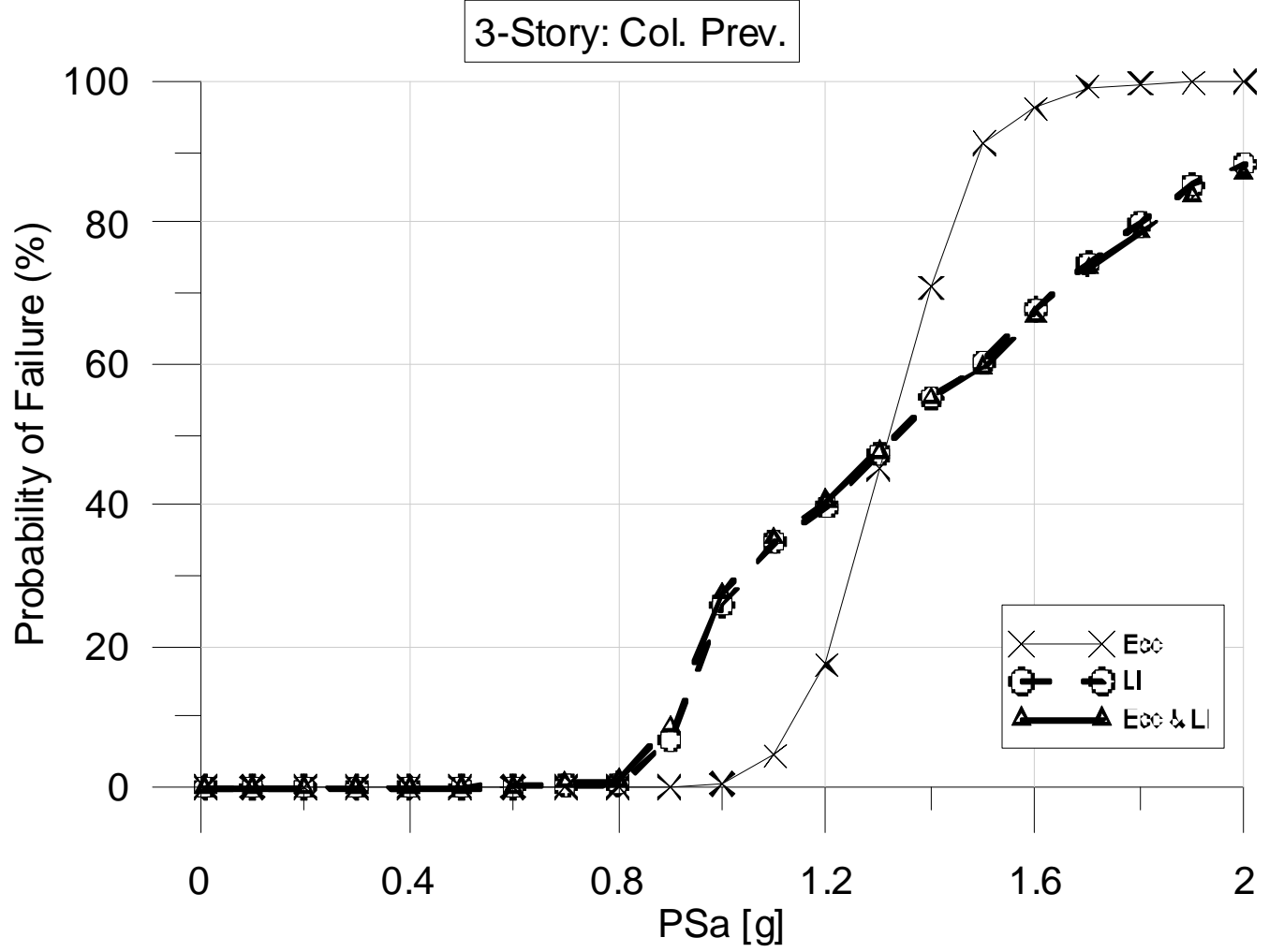

Figure E.6: Non-regression fragility curves collapse prevention comparison, 3-story 


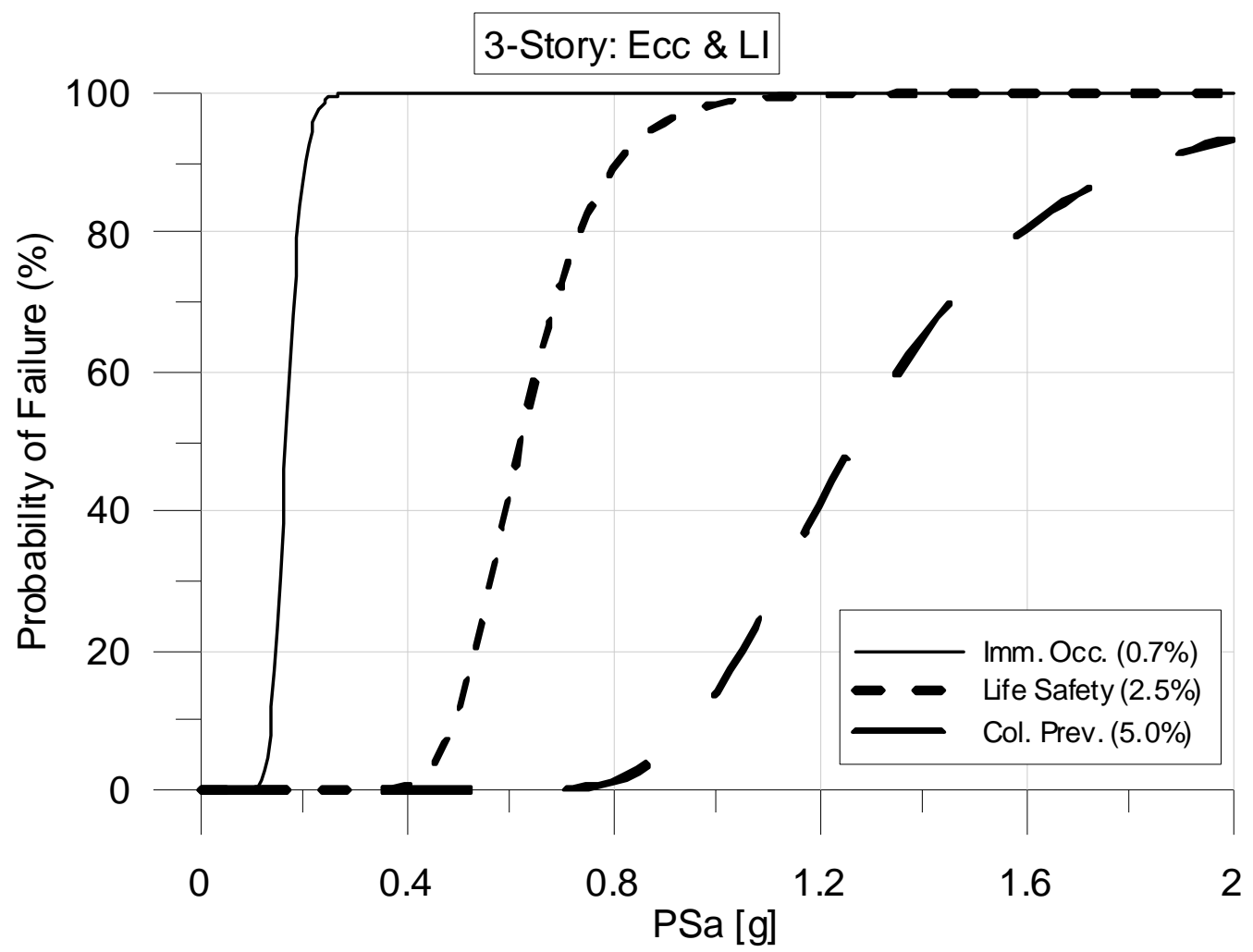

Figure E.7: Regression fragility curves for load eccentricity and intensity, 3-story

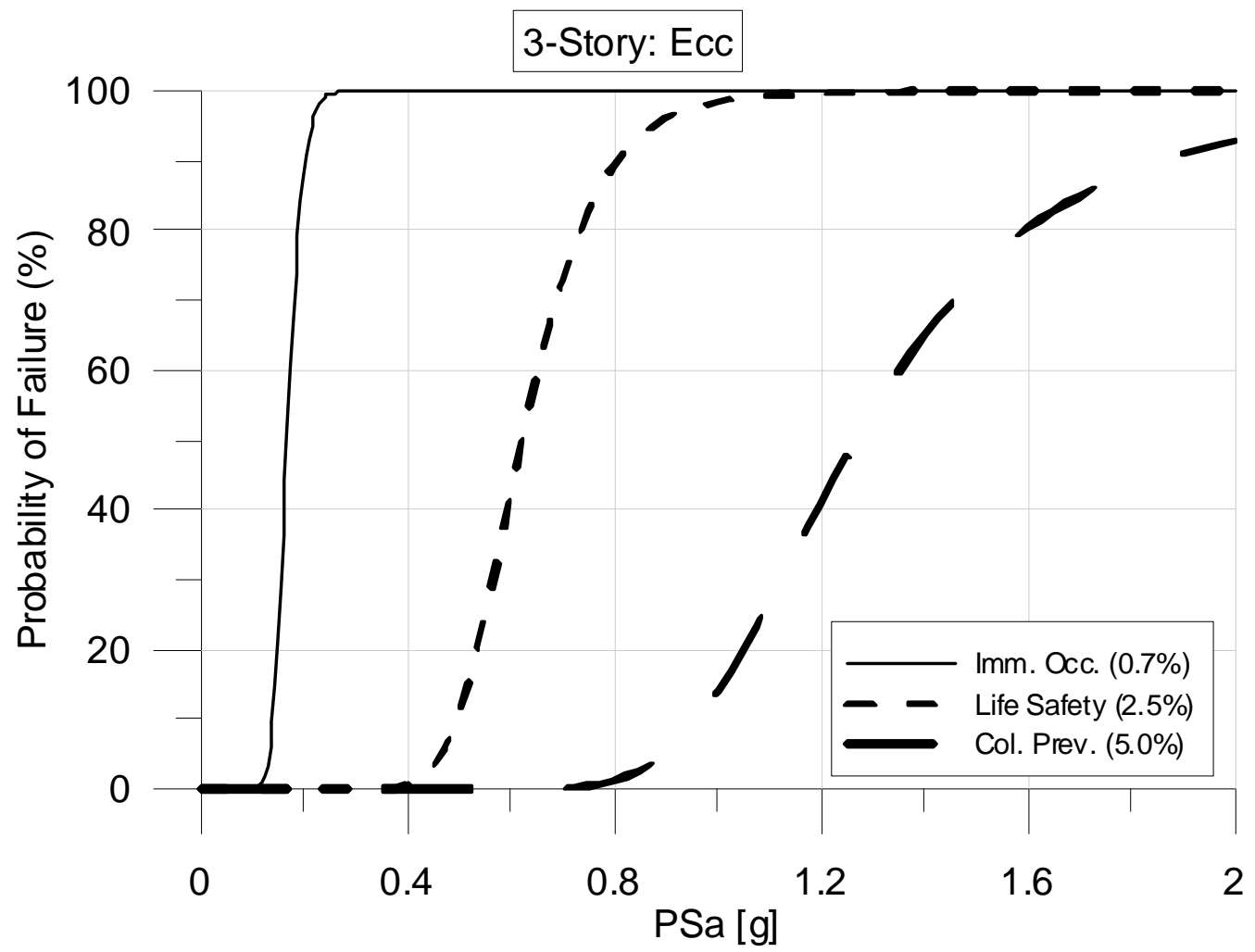

Figure E.8: Regression fragility curves for load eccentricity, 3-story 


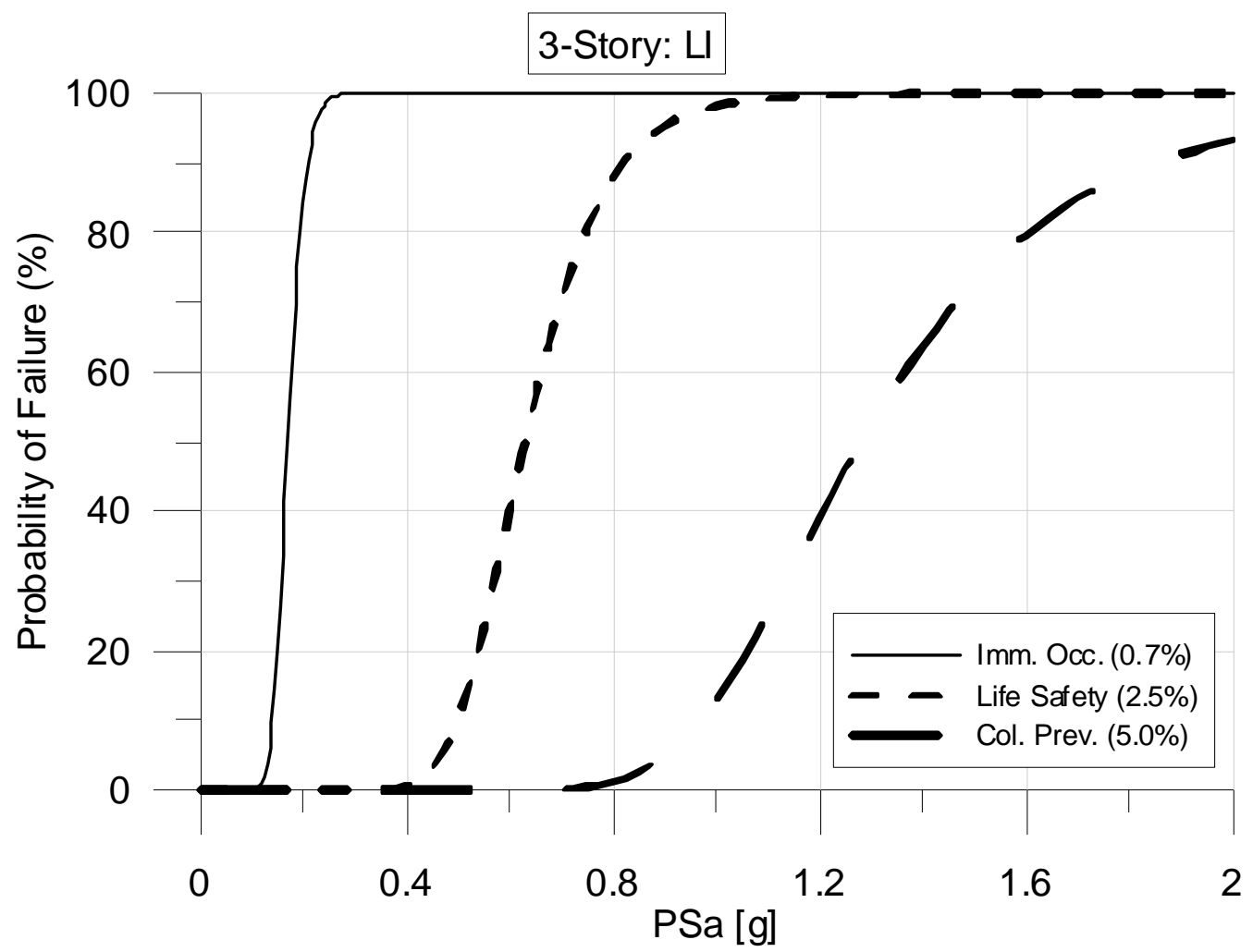

Figure E.9: Regression fragility curves for load intensity, 3-story

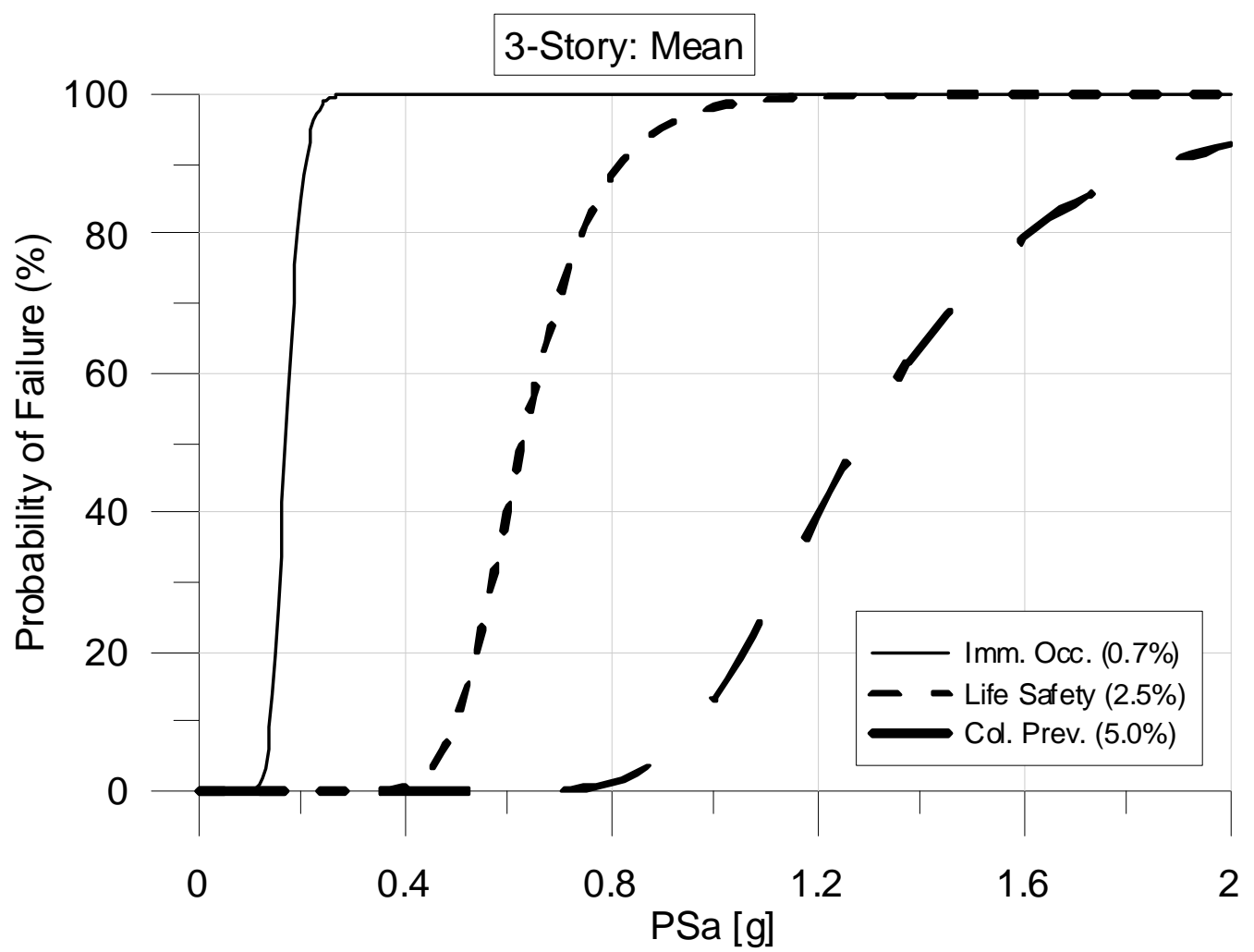

Figure E.10: Regression fragility curves for mean values of eccentricity and intensity, 3-story 


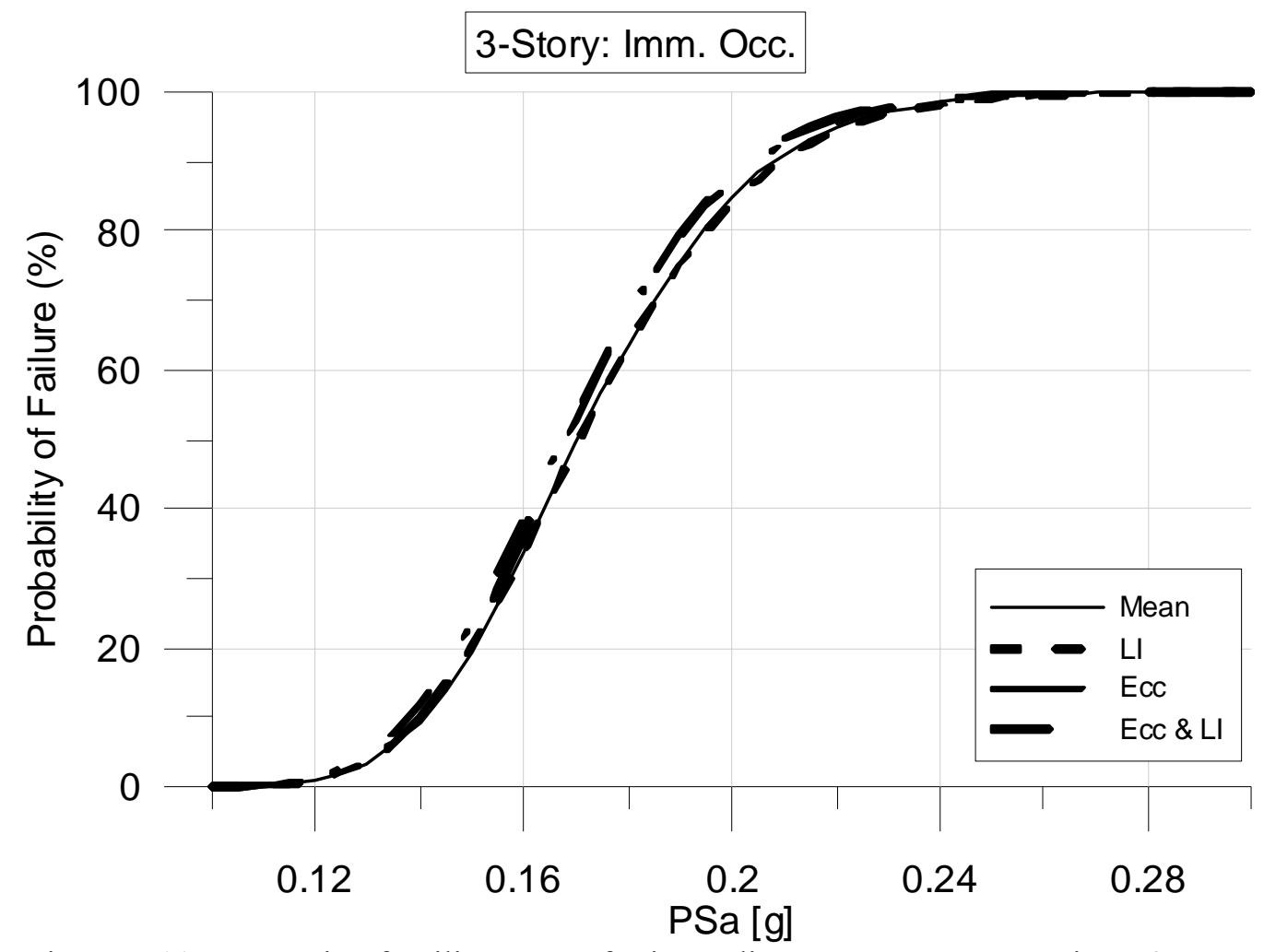

Figure E.11: Regression fragility curves for immediate occupancy comparison, 3-story

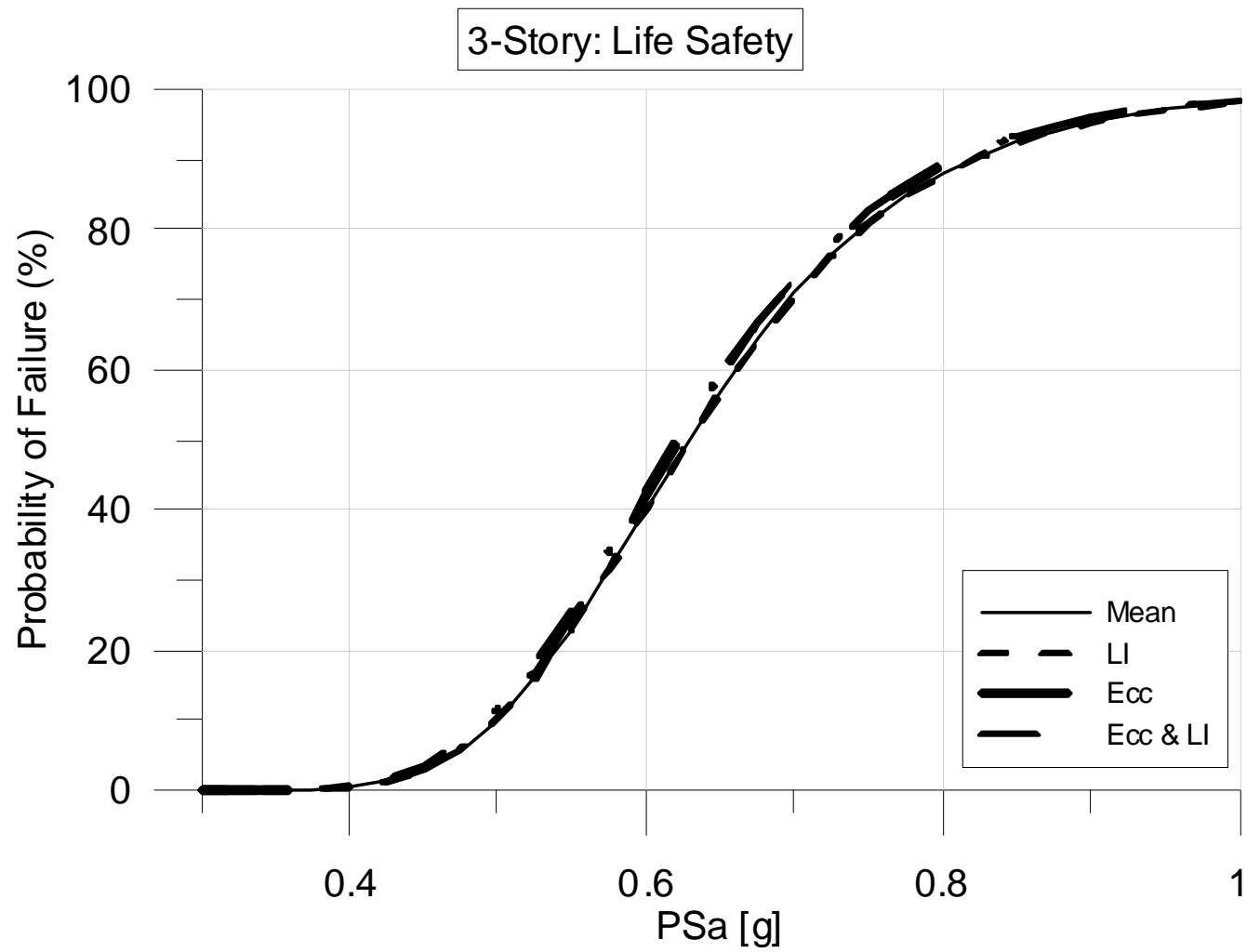

Figure E.12: Regression fragility curves for life safety comparison, 3-story 


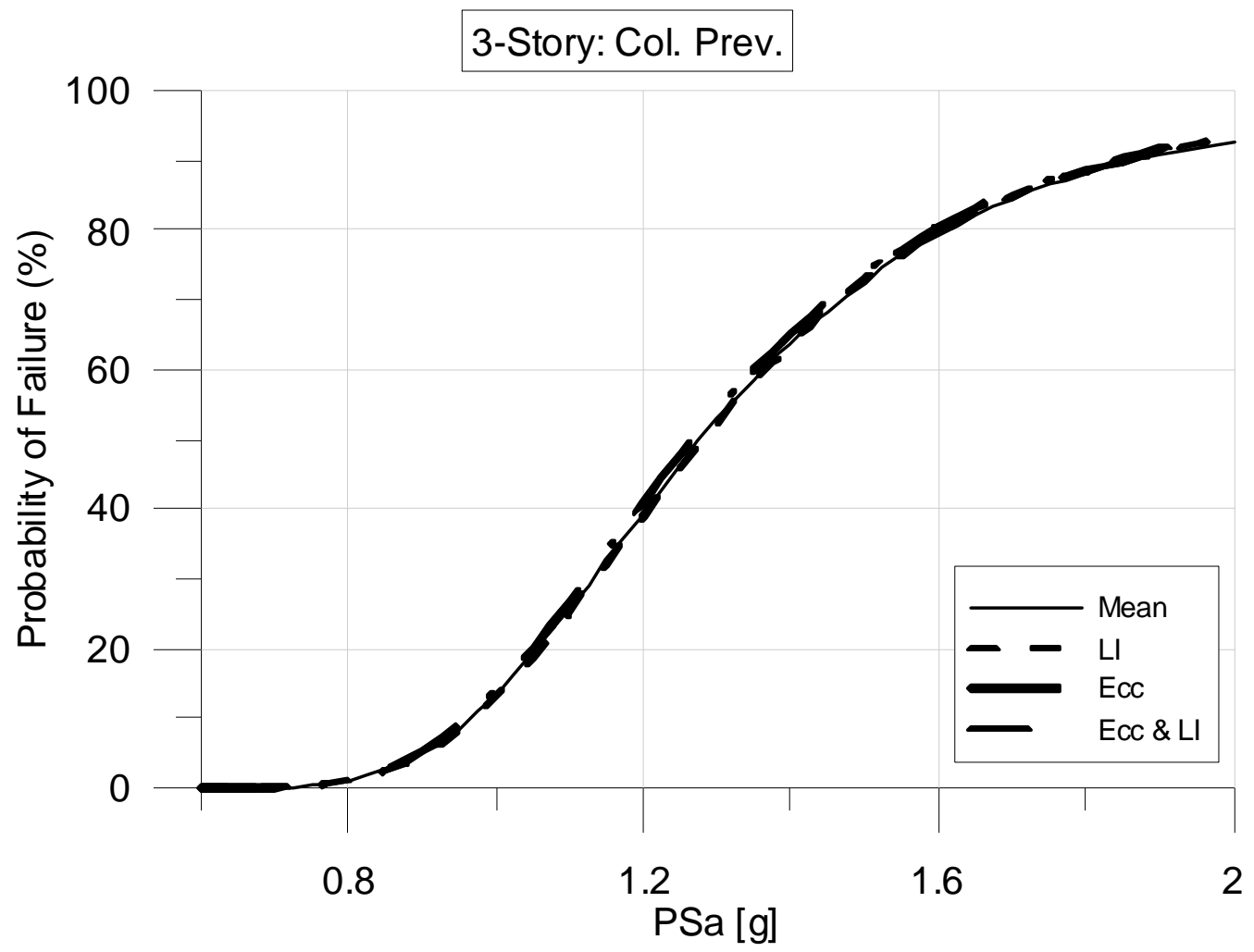

Figure E.13: Regression fragility curves for collapse prevention comparison, 3-story

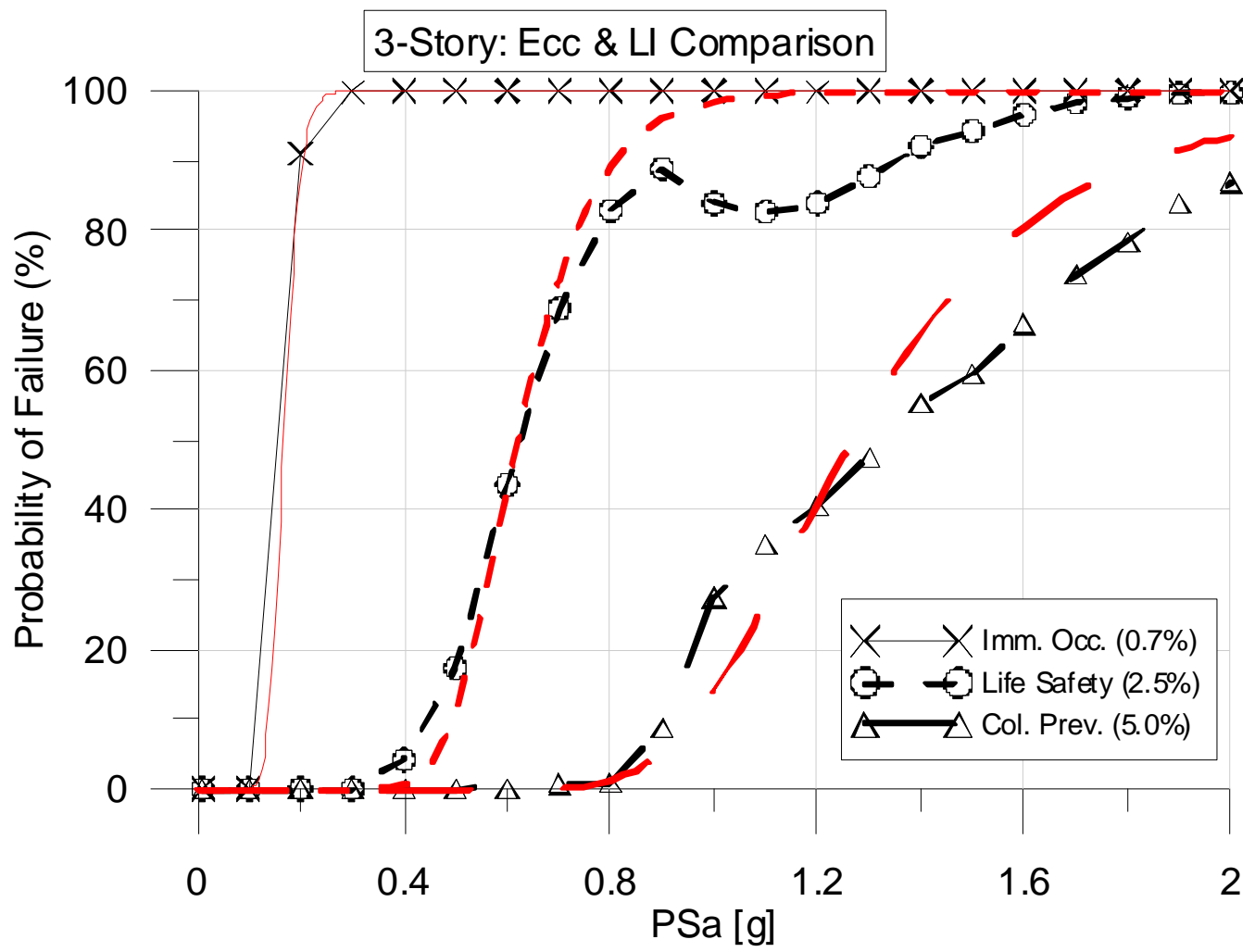

Figure E.14: Regression and non-regression fragility curve comparison for load eccentricity and intensity, 3-story 


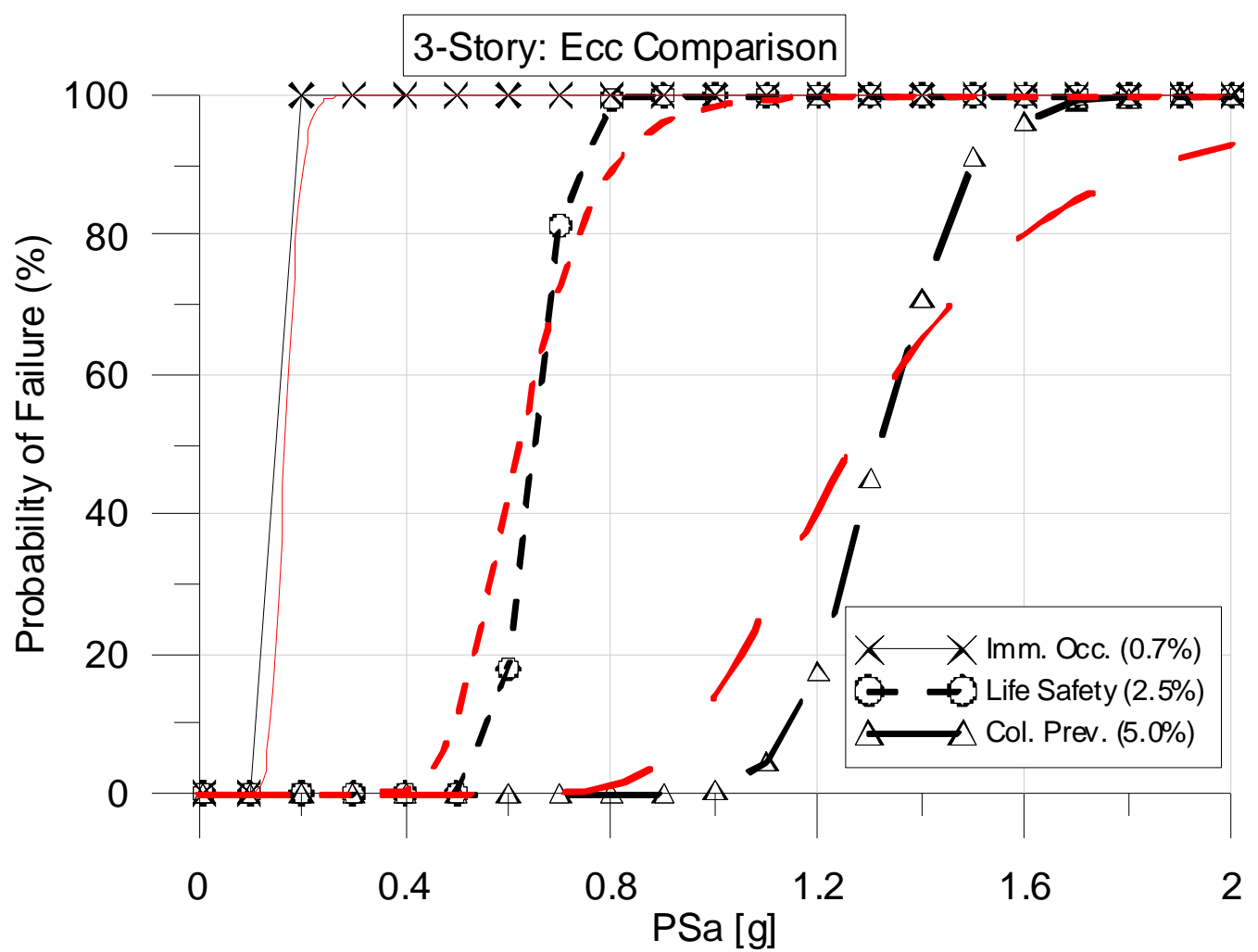

Figure E.15: Regression and non-regression fragility curve comparison for load eccentricity, 3-story

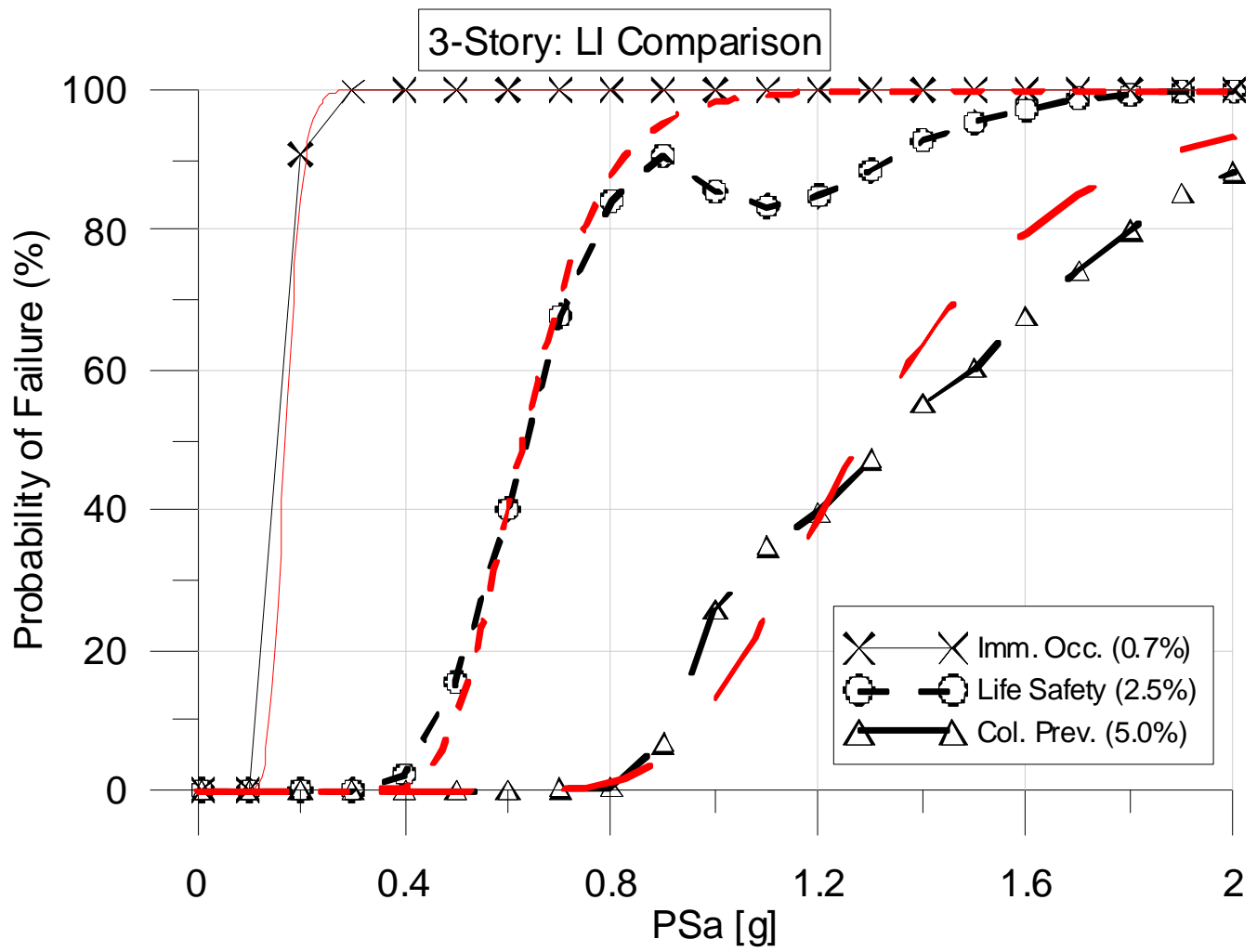

Figure E.16: Regression and non-regression fragility curve comparison for load intensity, 3 -story 


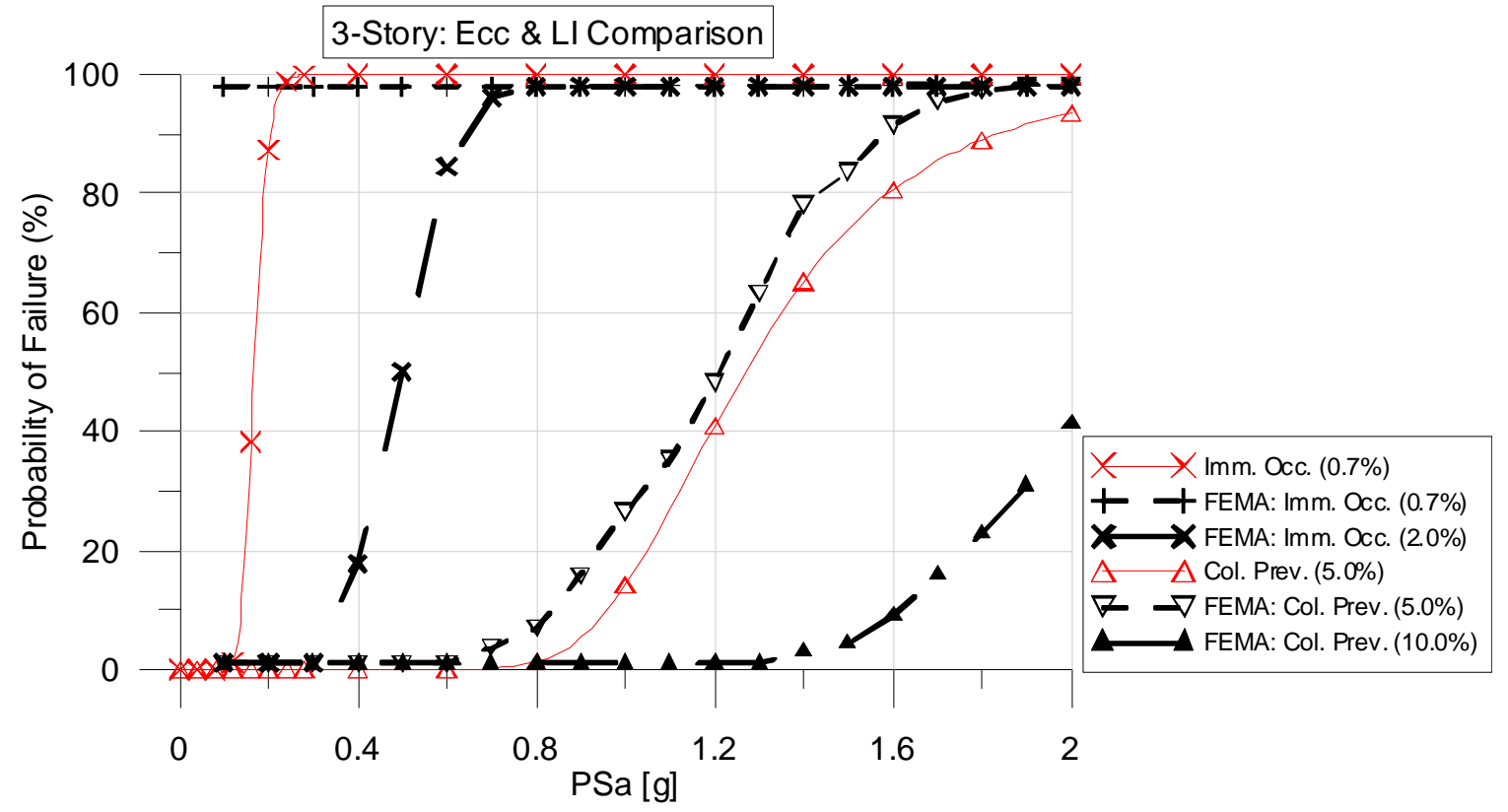

Figure E.17: Regression and FEMA comparison for load eccentricity and intensity, 3-story

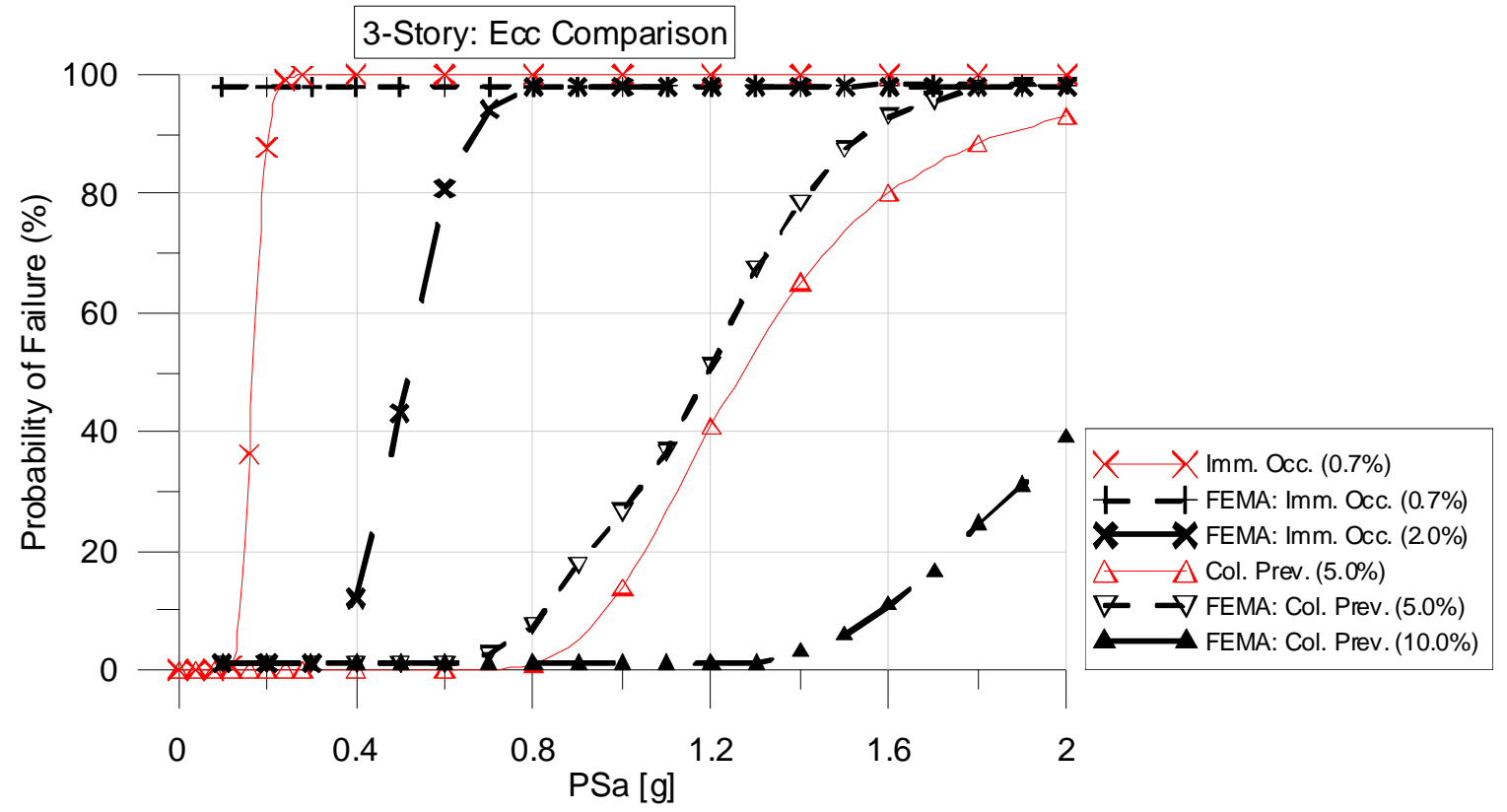

Figure E.18: Regression and FEMA comparison for load eccentricity, 3-story 


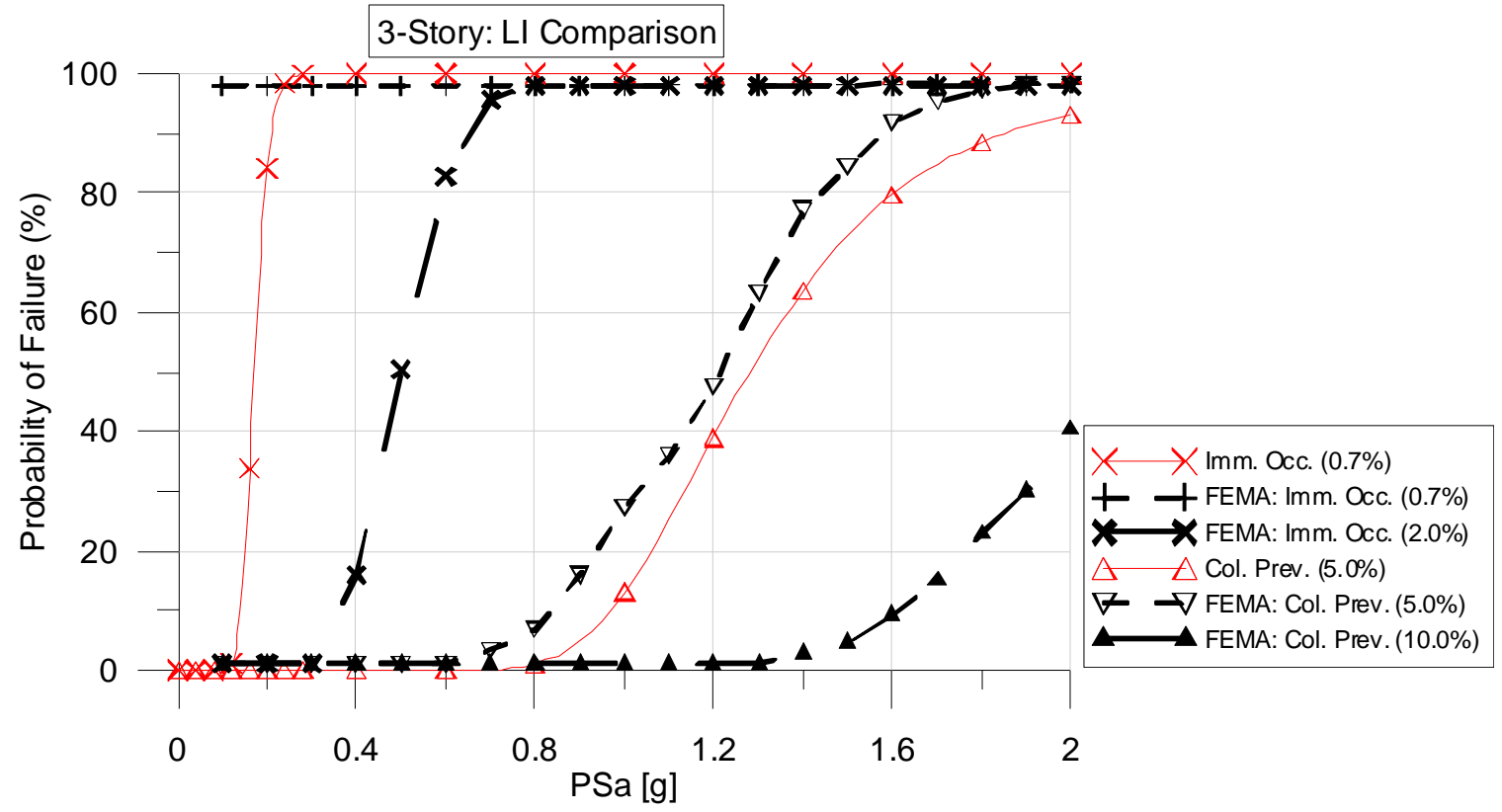

Figure E.19: Regression and FEMA comparison for load intensity, 3-story 


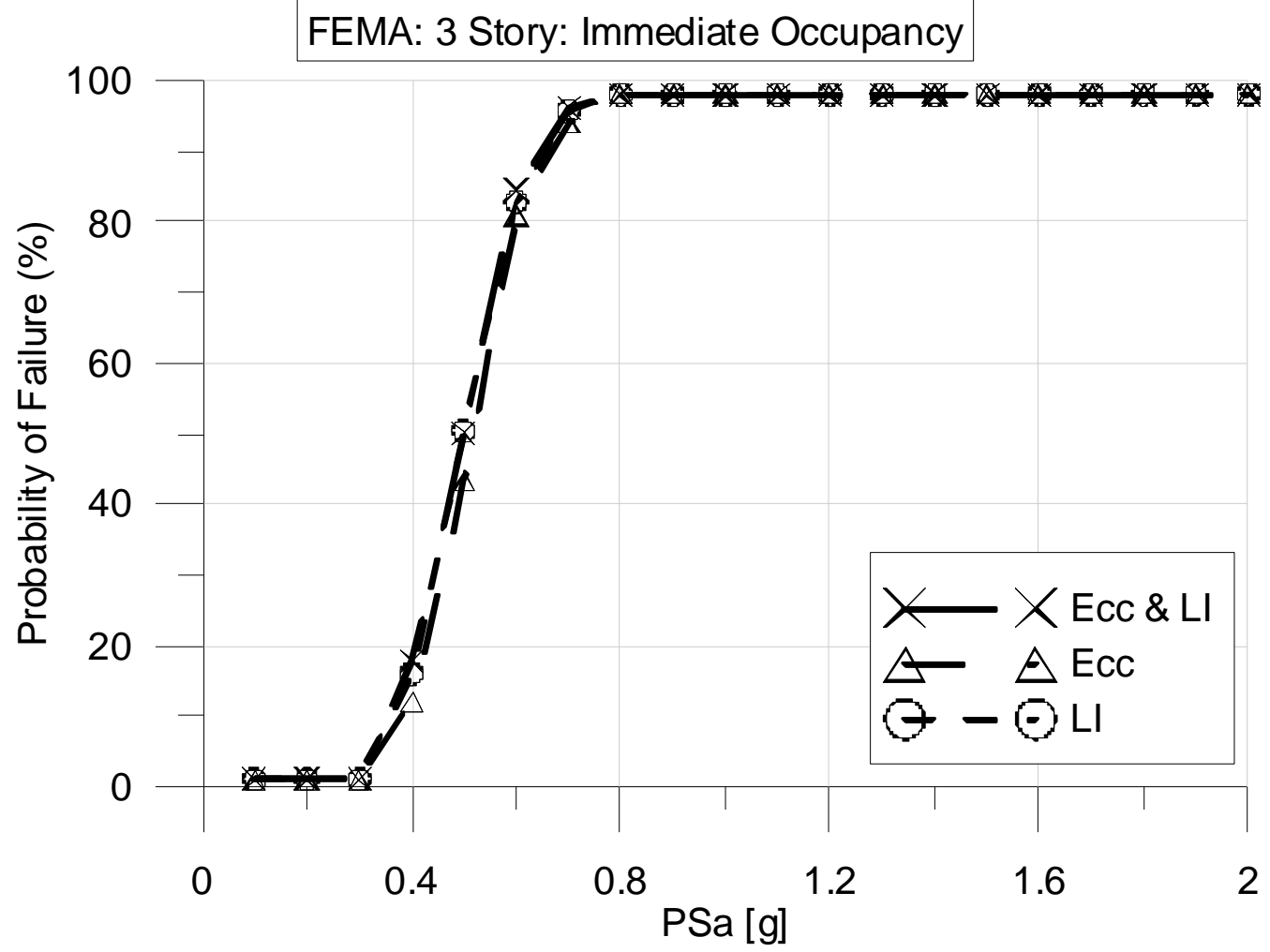

Figure E.20: FEMA 350 fragility curves for immediate occupancy comparison, 3-story

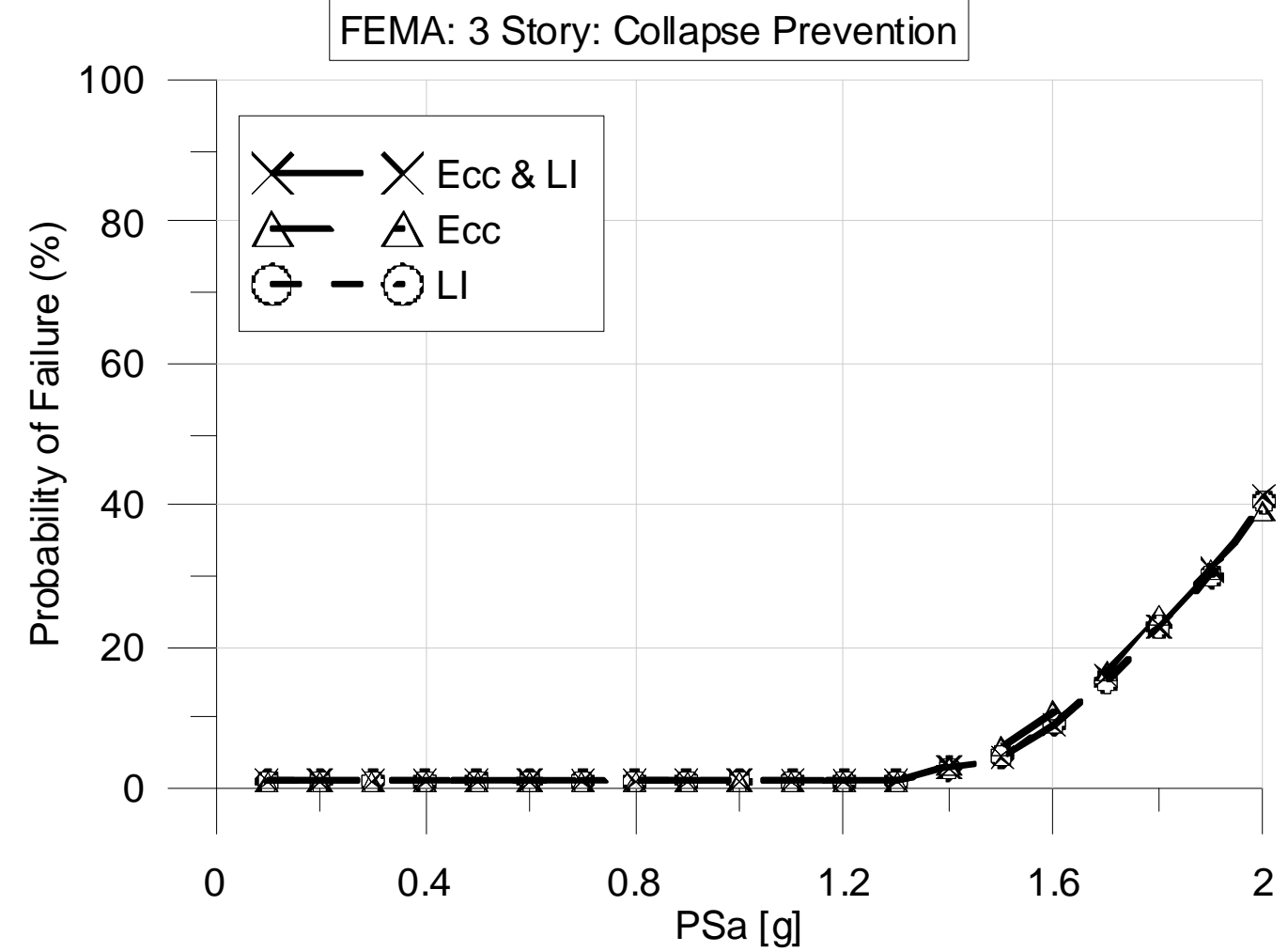

Figure E.21: FEMA 350 fragility curves for collapse prevention comparison, 3-story 


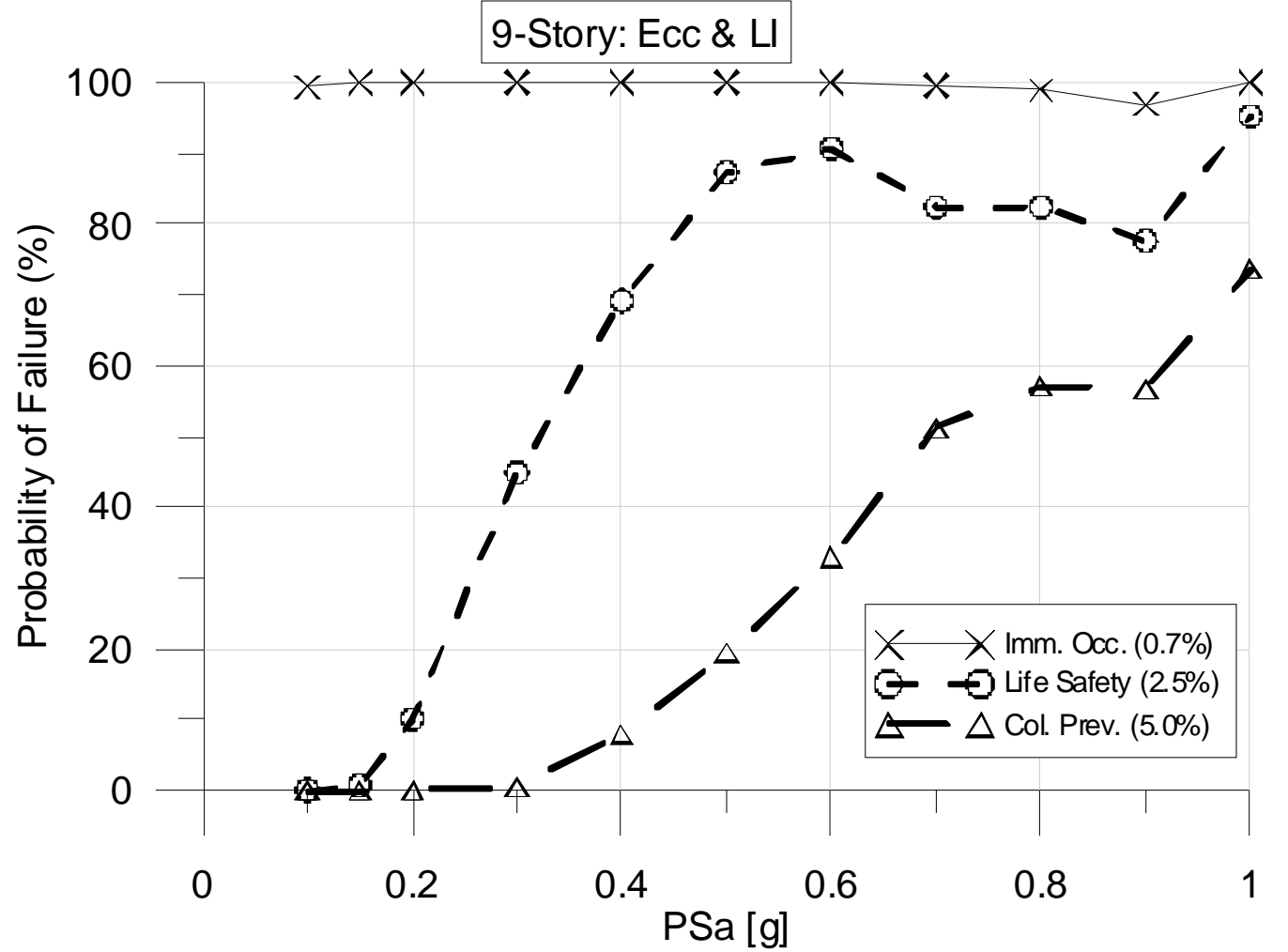

Figure E.22: Non-regression fragility curves for load eccentricity and intensity, 9-story

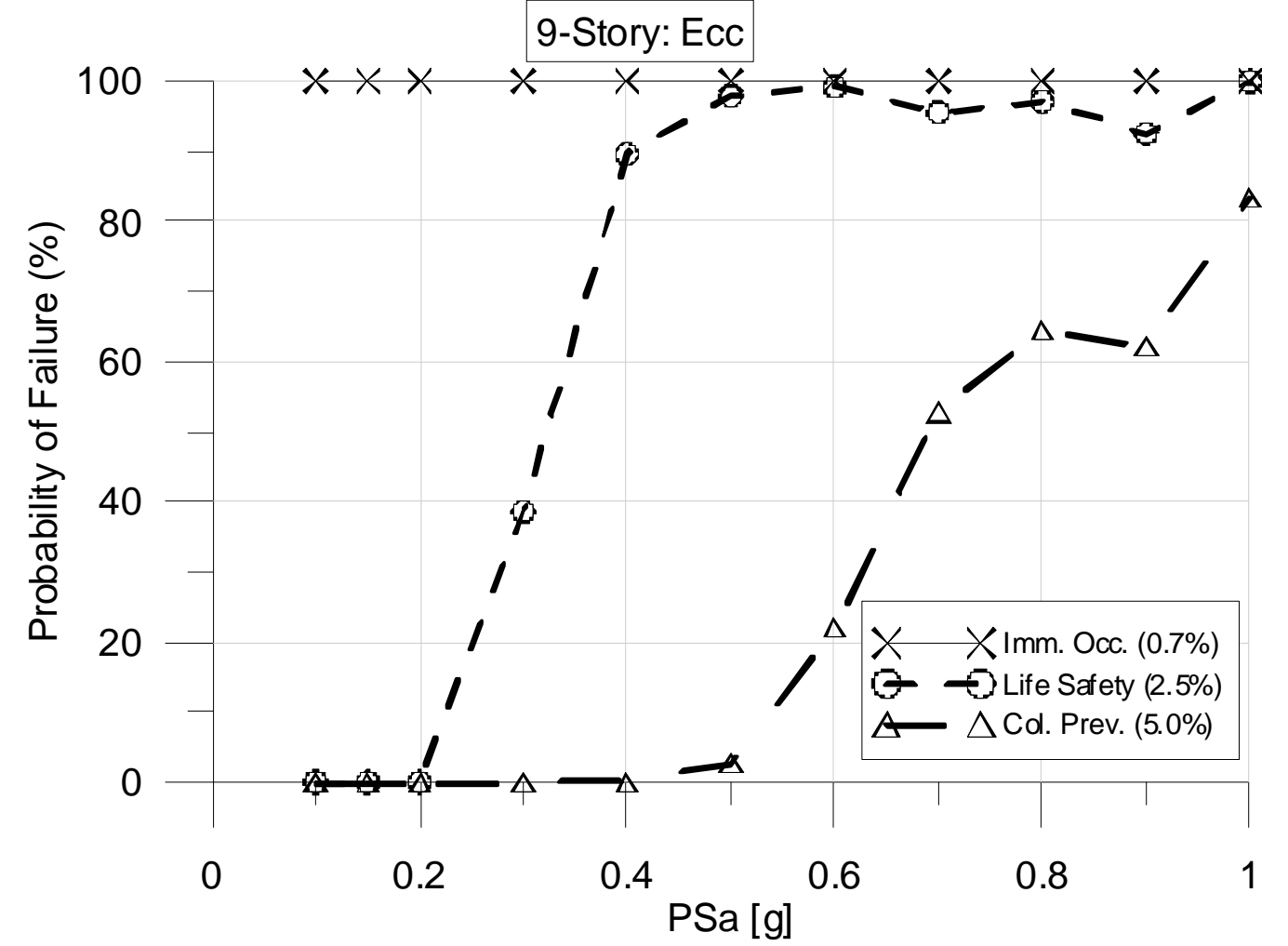

Figure E.23: Non-regression fragility curves for load eccentricity, 9-story 


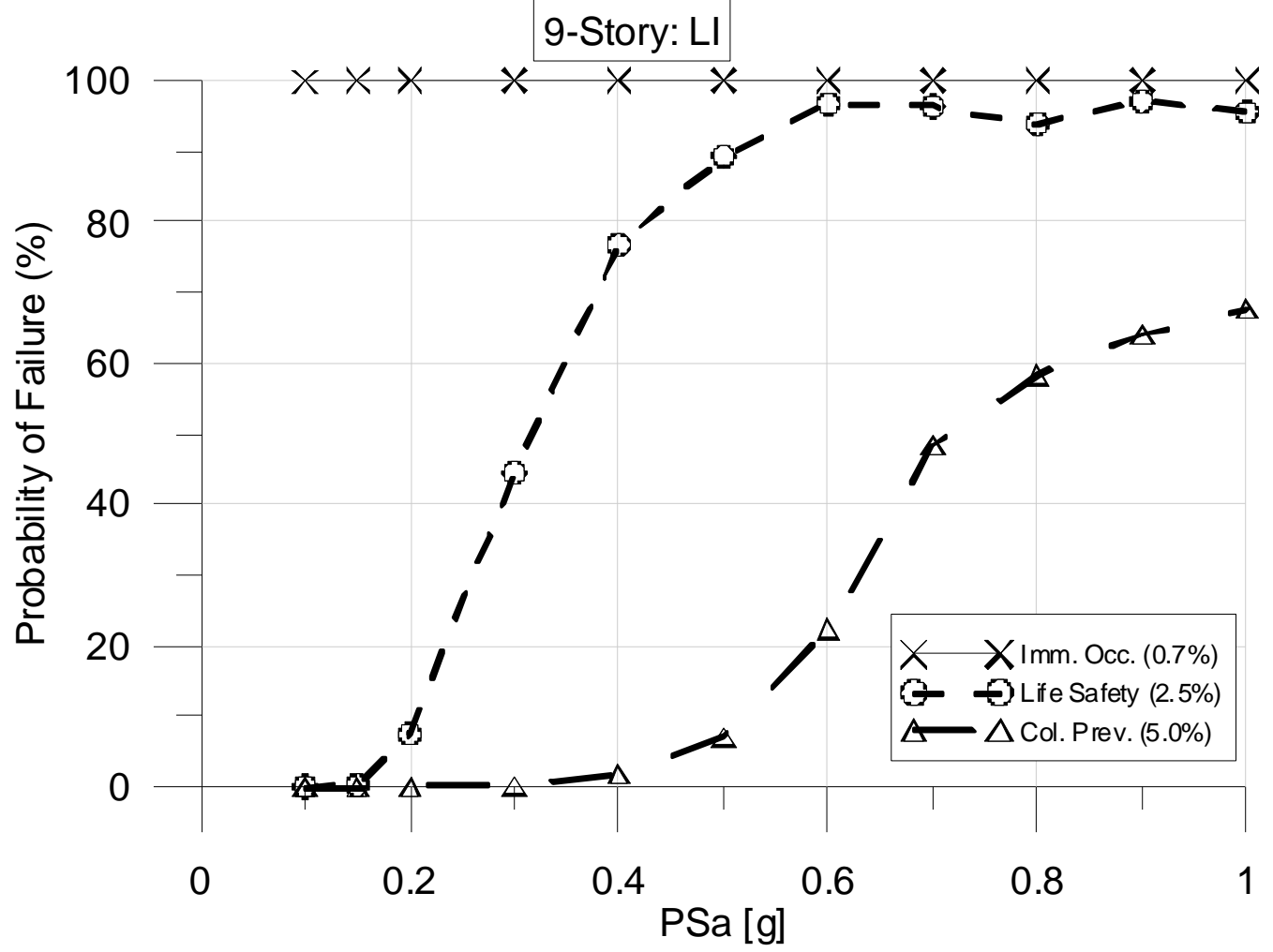

Figure E.24: Non-regression fragility curves for load intensity, 9-story

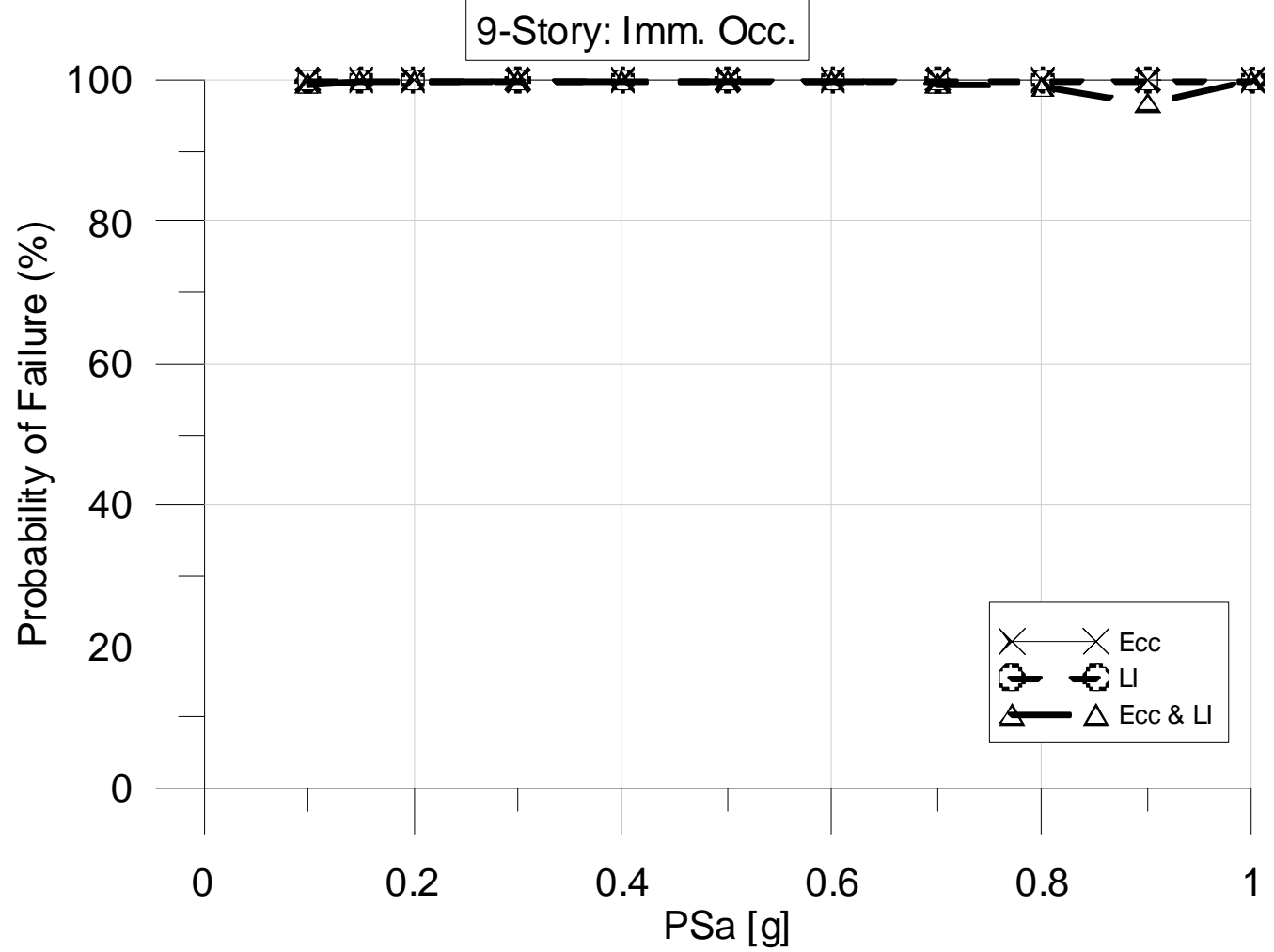

Figure E.25: Non-regression fragility curves immediate occupancy comparison, 9-story 


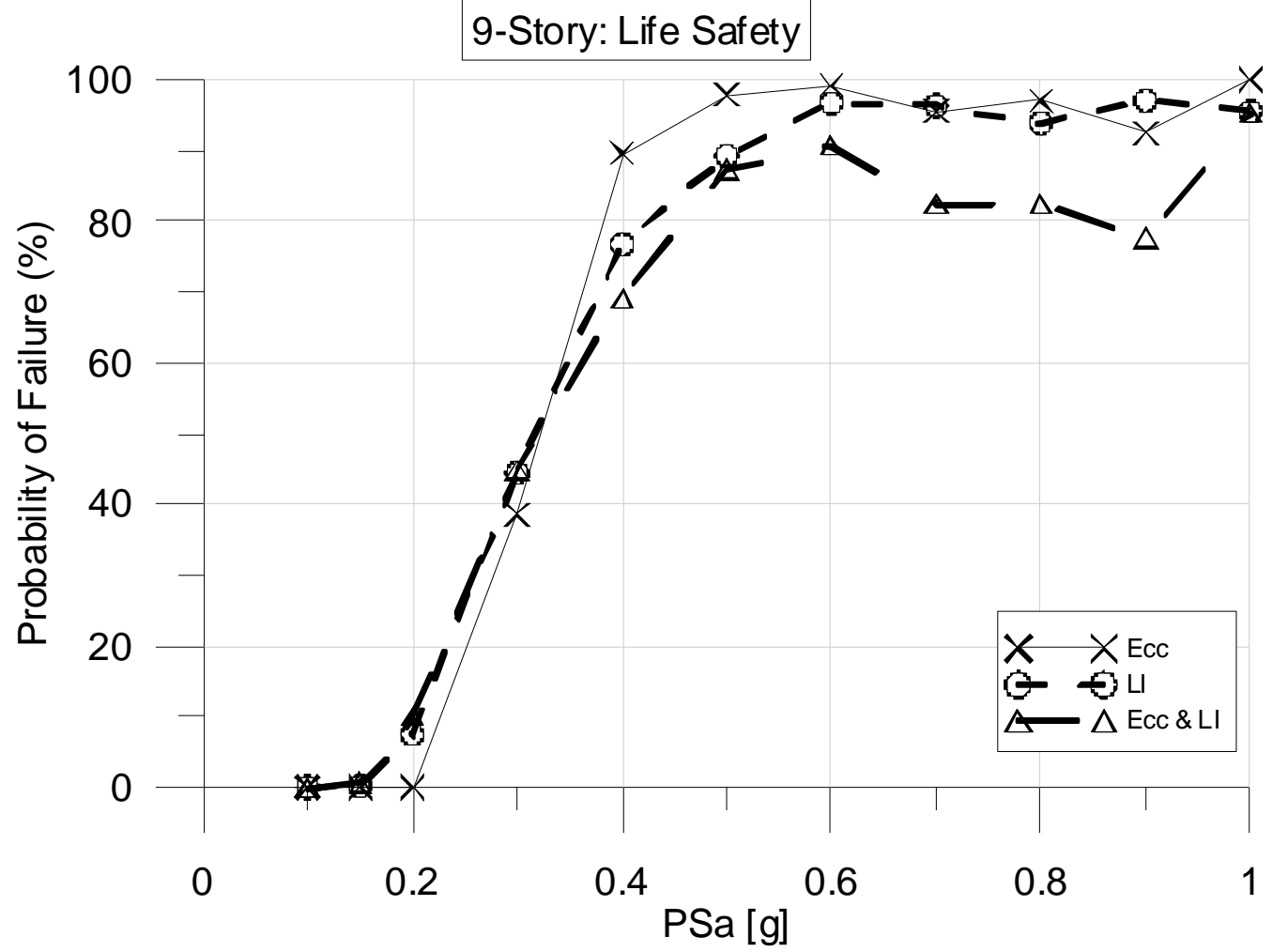

Figure E.26: Non-regression fragility curves life safety comparison, 9-story

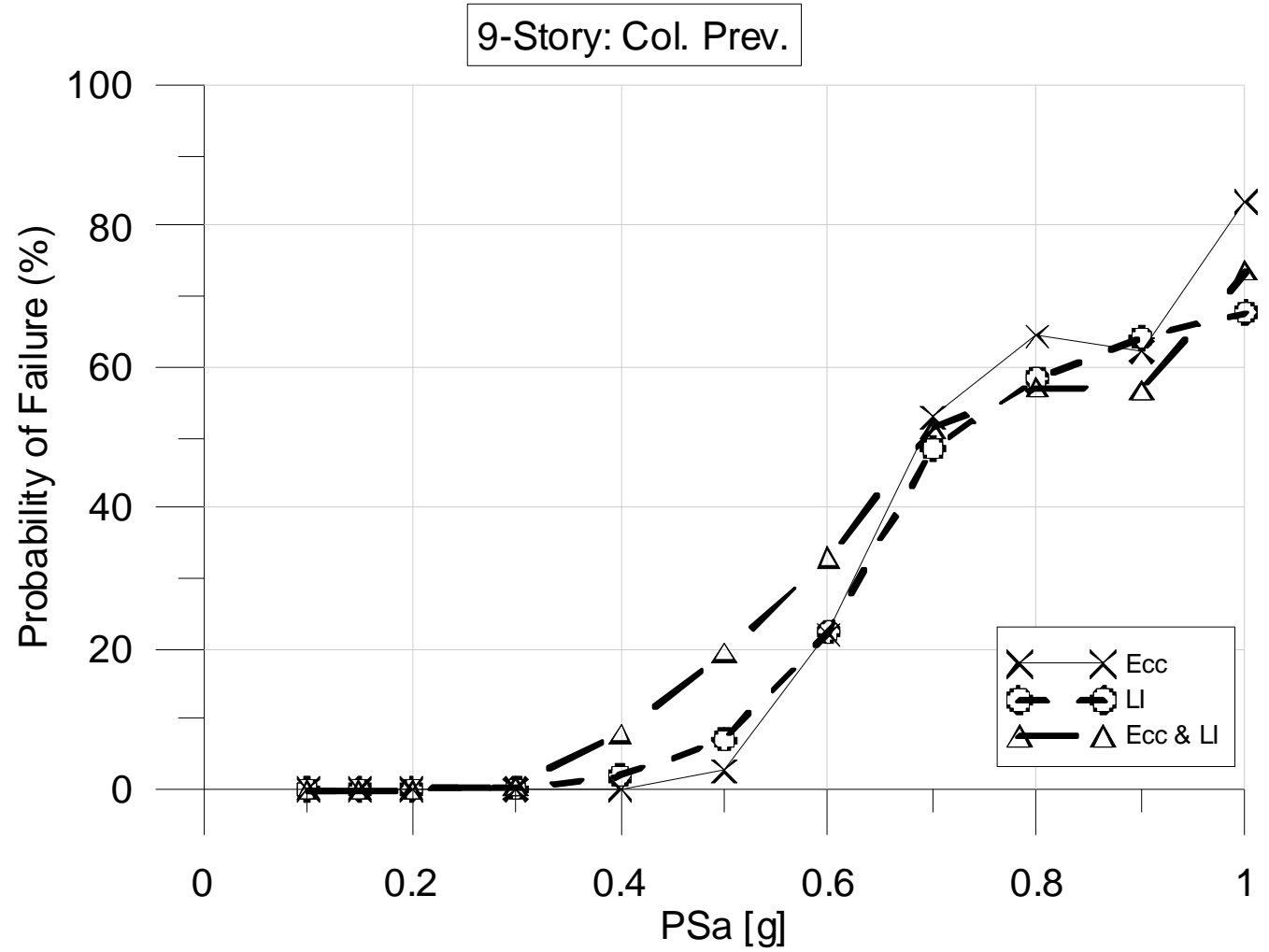

Figure E.27: Non-regression fragility curves collapse prevention comparison, 9-story 


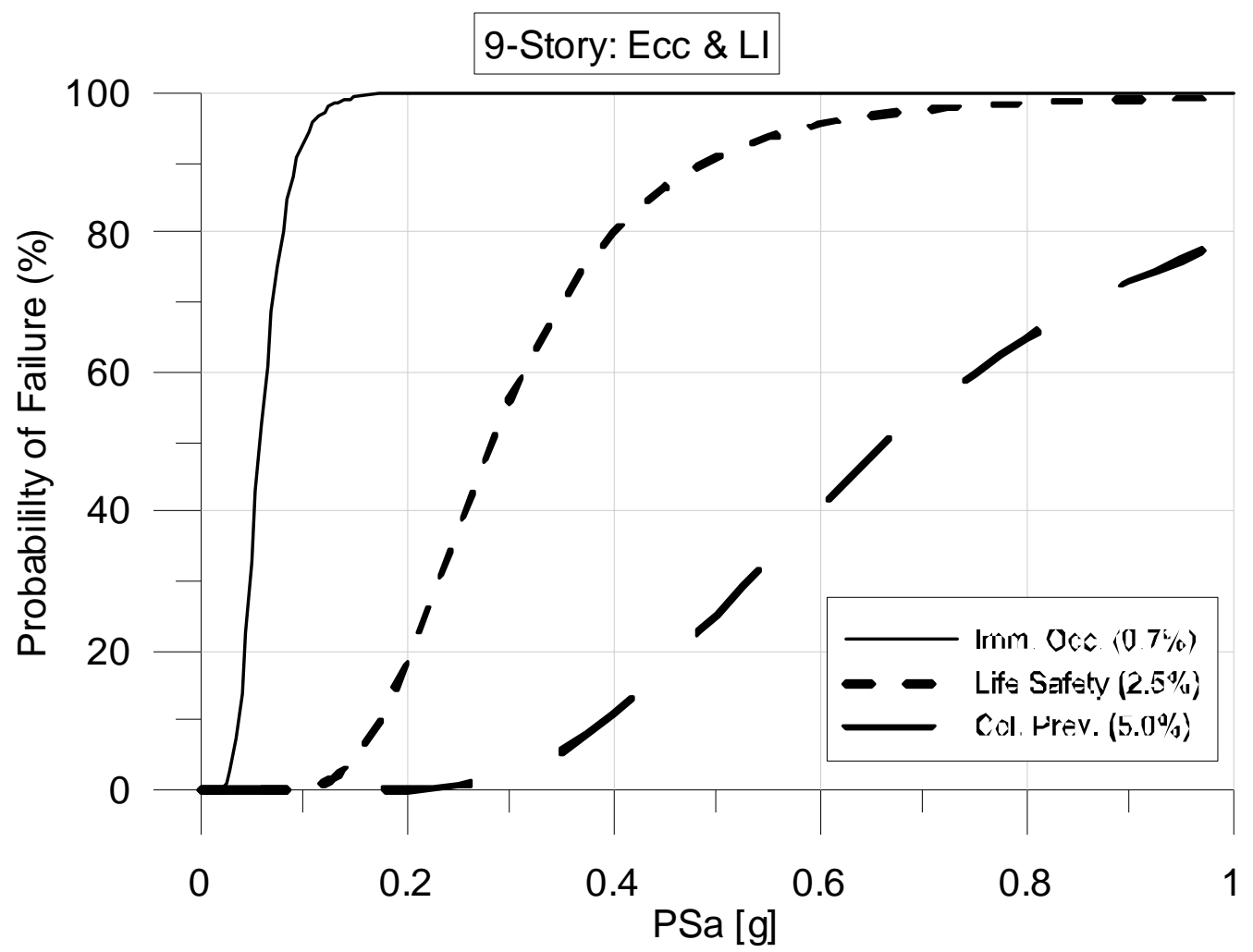

Figure E.28: Regression fragility curves for load eccentricity and intensity, 9-story

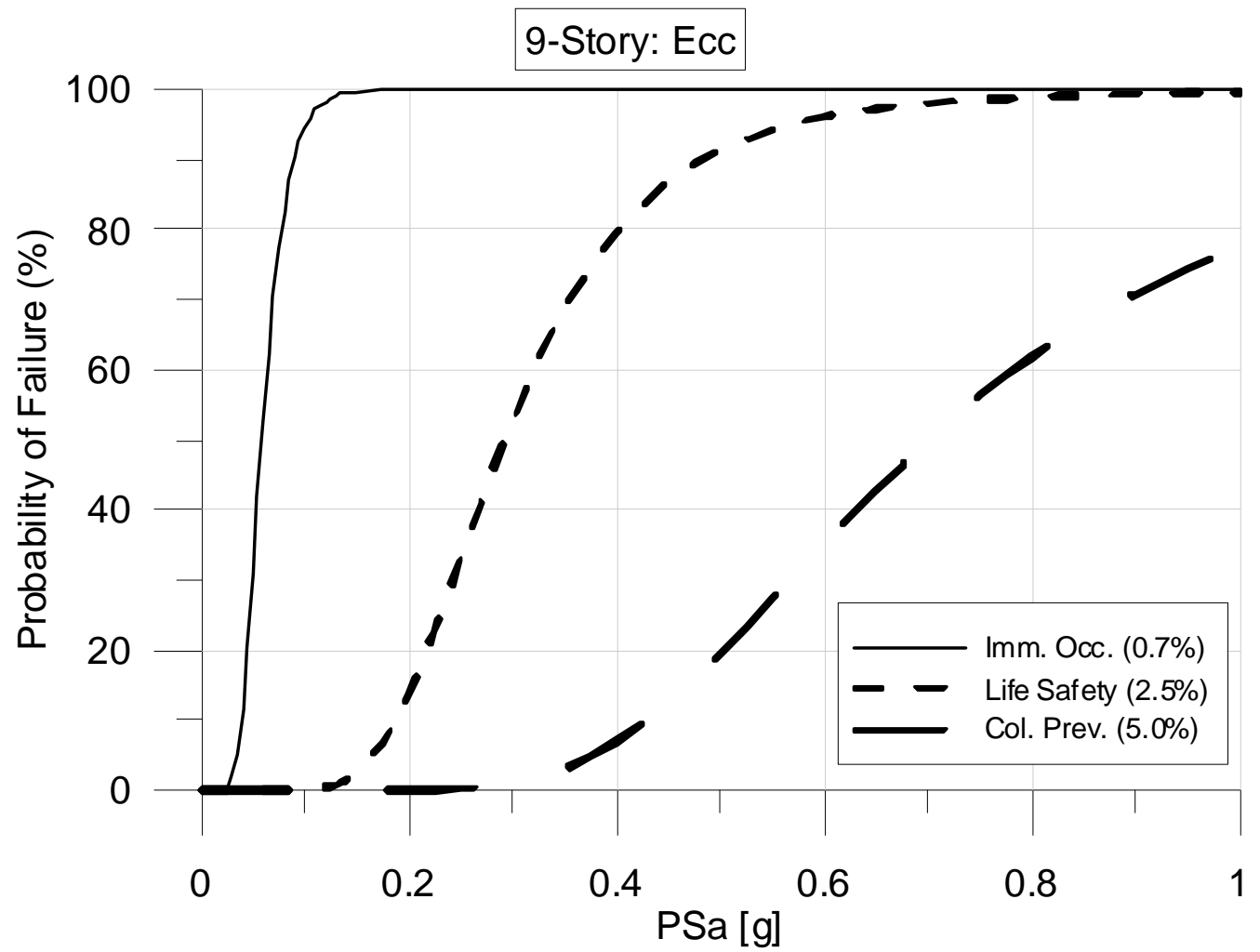

Figure E.29: Regression fragility curves for load eccentricity, 9-story 


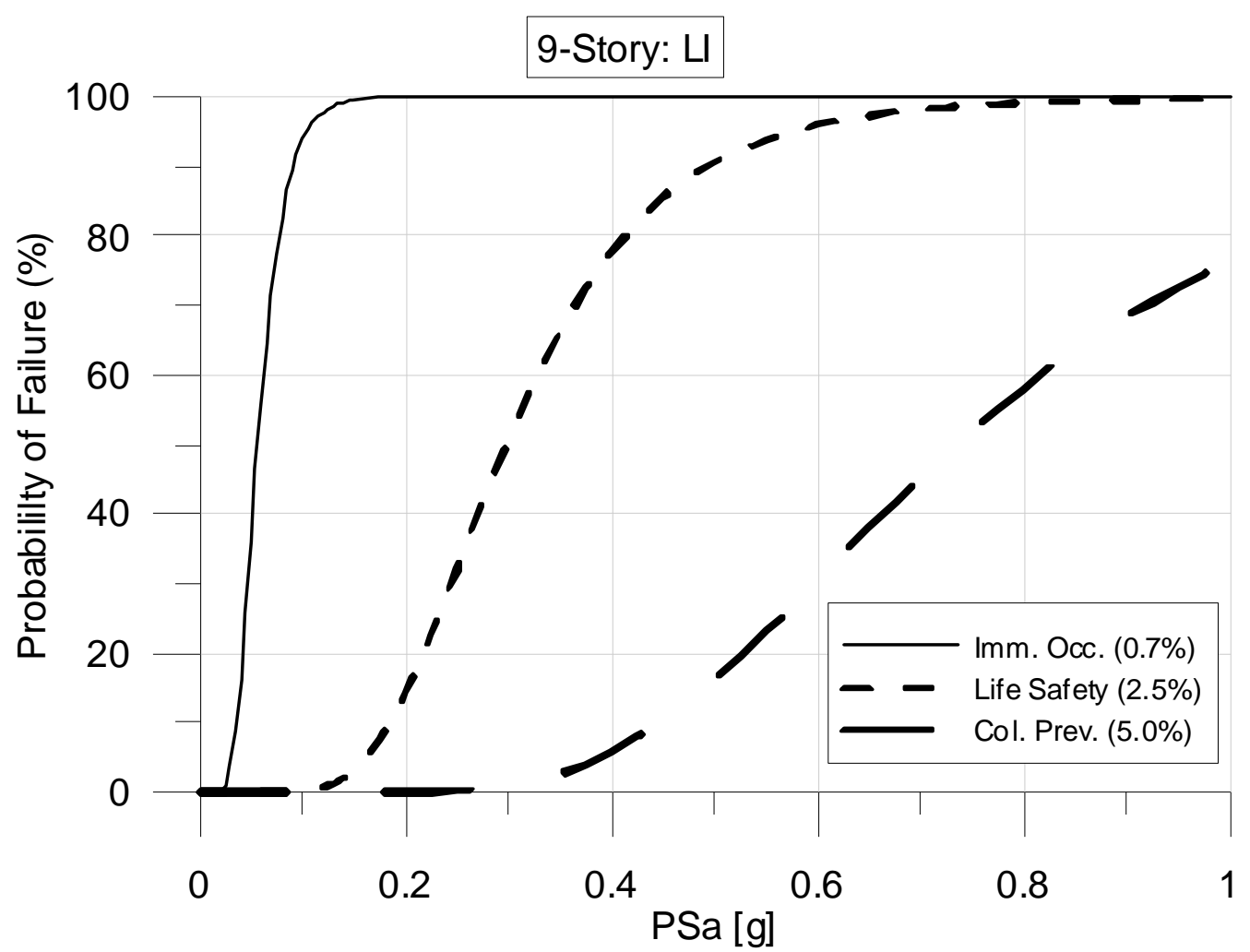

Figure E.30: Regression fragility curves for load intensity, 9-story

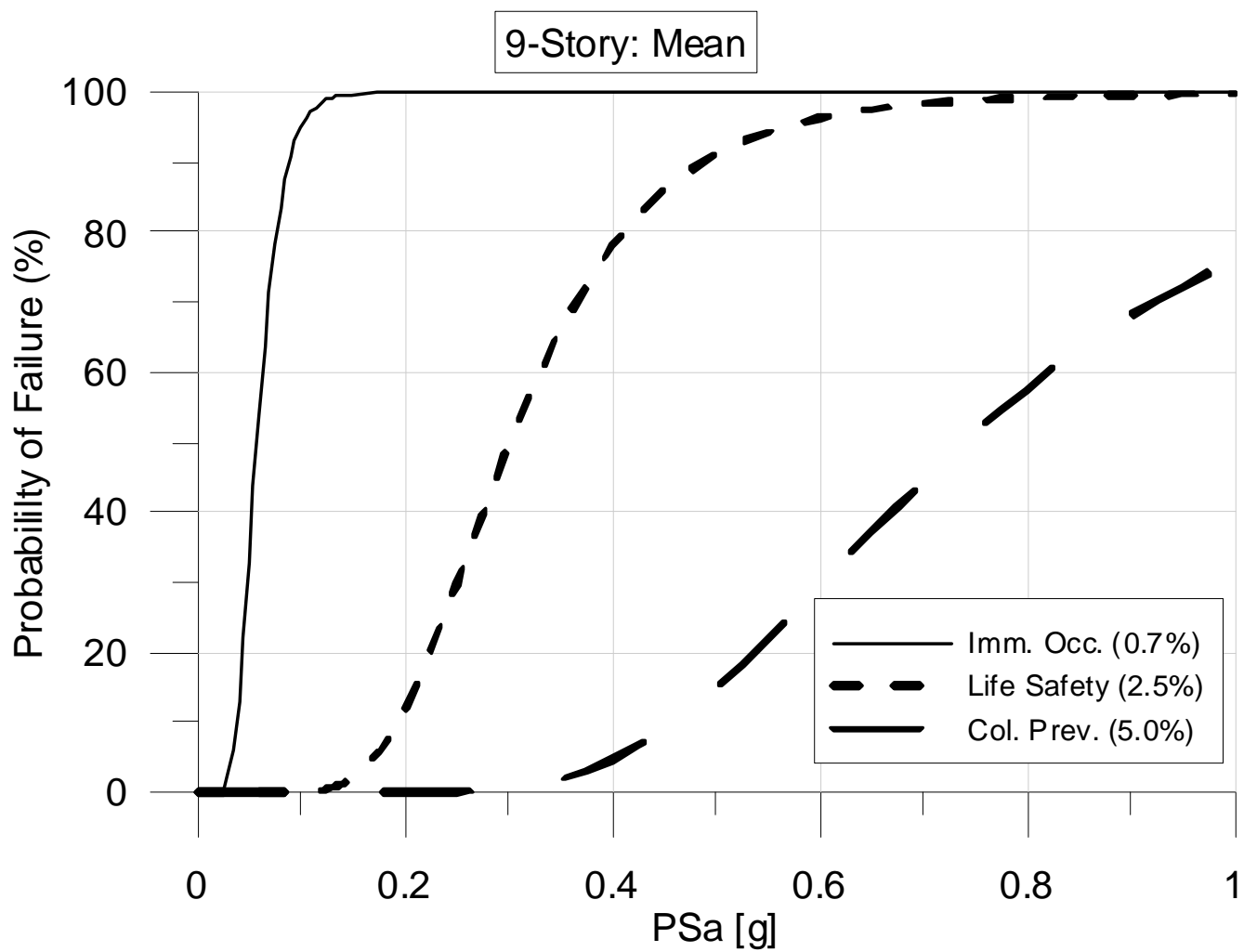

Figure E.31: Regression fragility curves for mean values of eccentricity and intensity, 9-story 


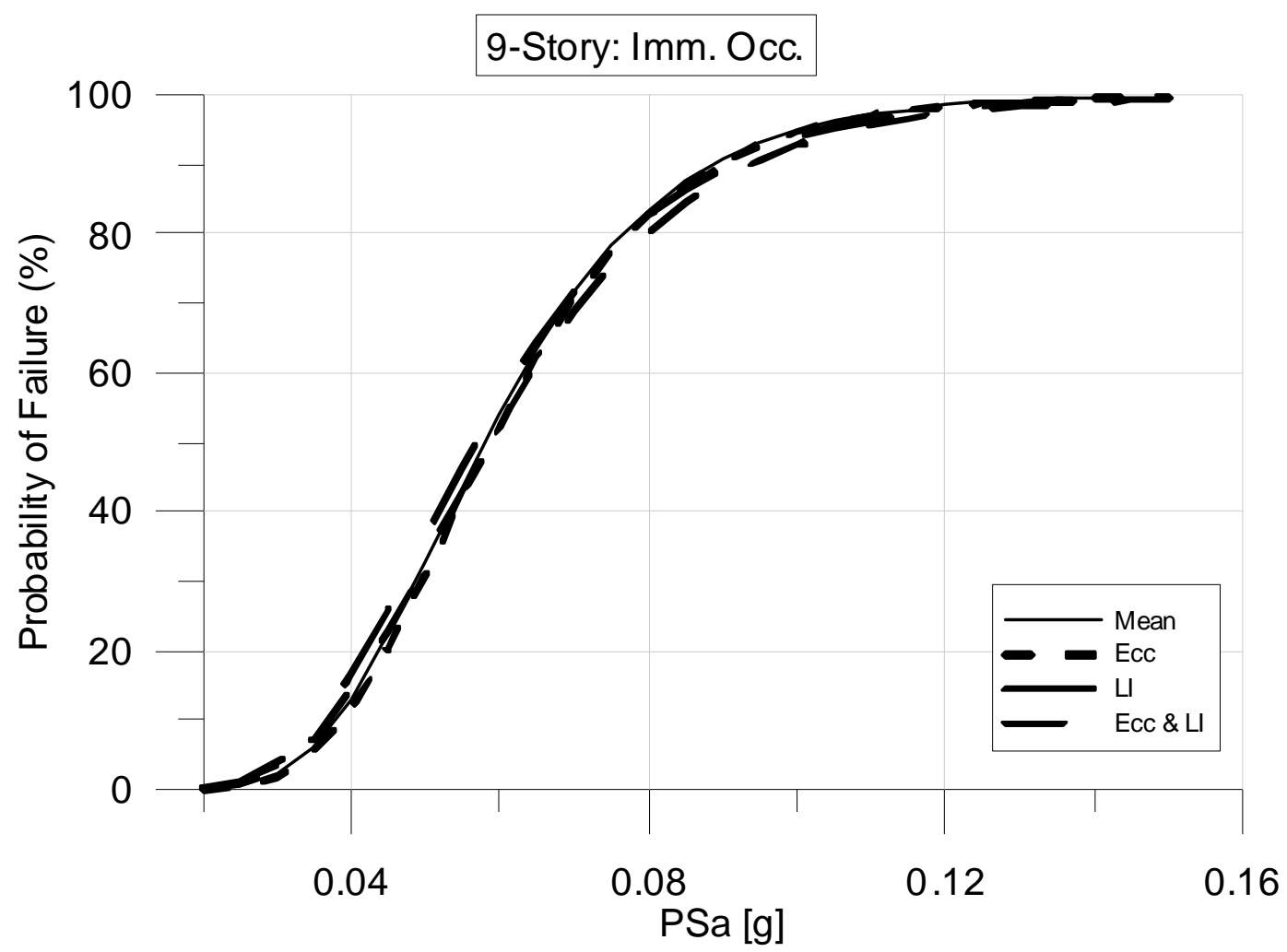

Figure E.32: Regression fragility curves for immediate occupancy comparison, 9-story

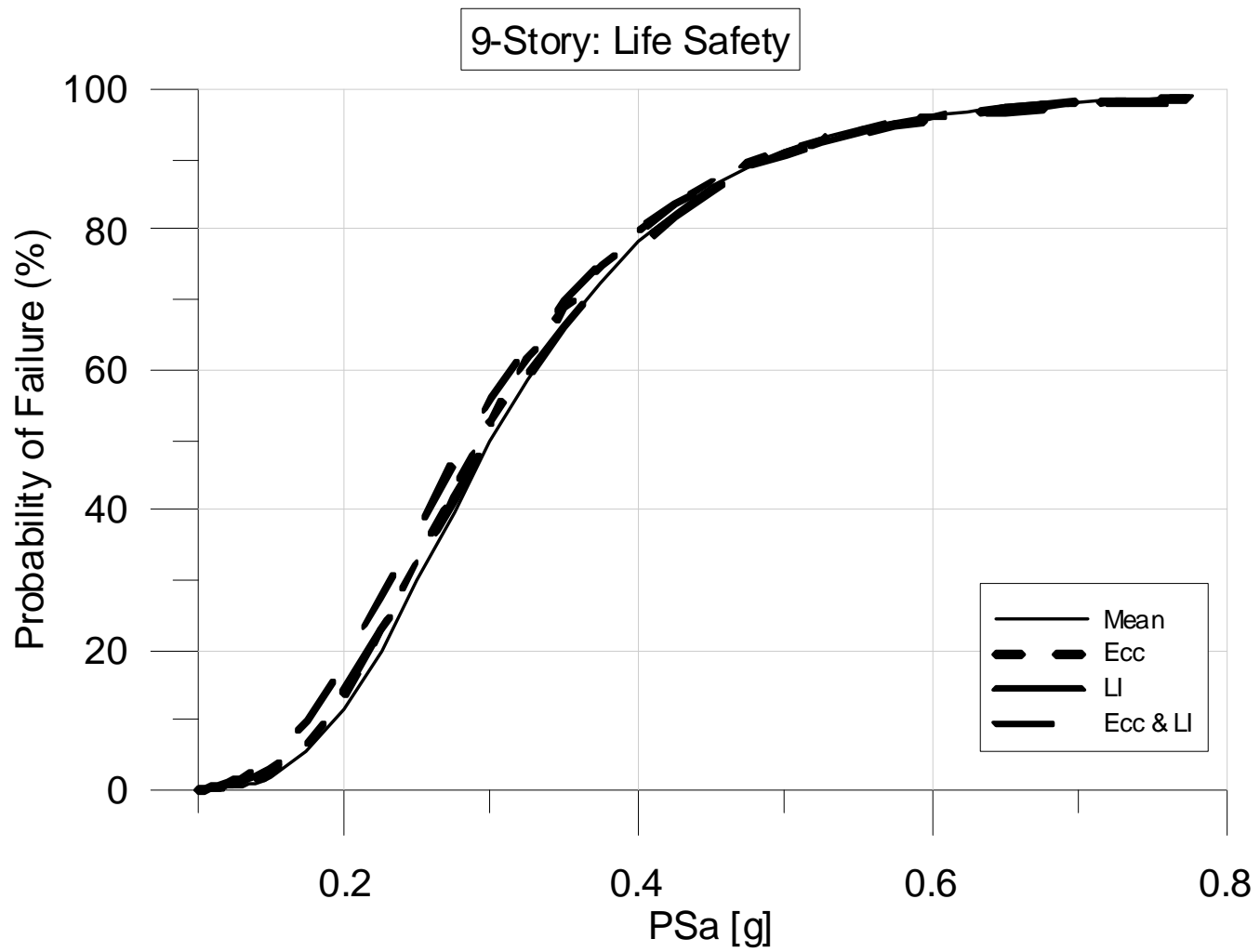

Figure E.33: Regression fragility curves for life safety comparison, 9-story 


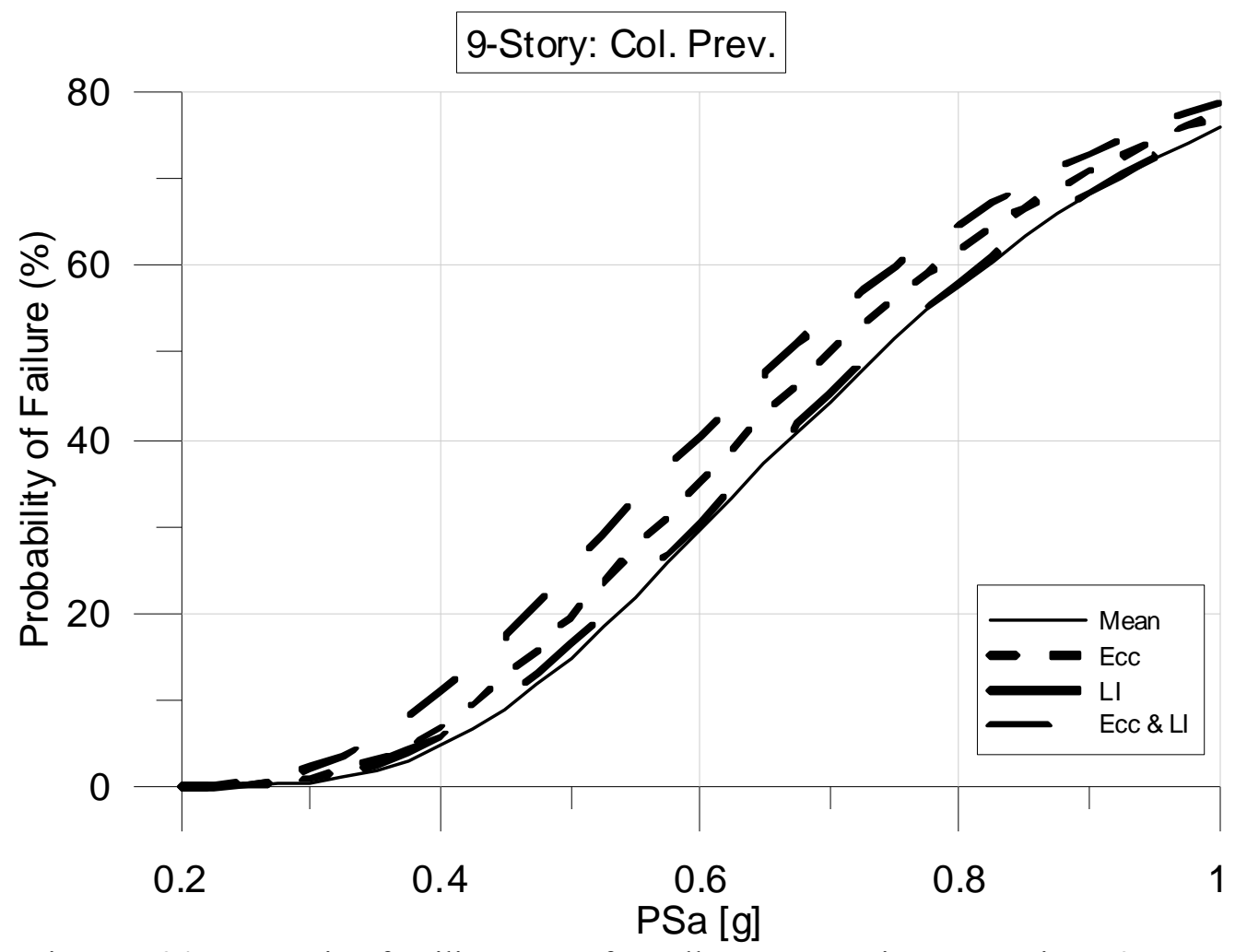

Figure E.34: Regression fragility curves for collapse prevention comparison, 9-story

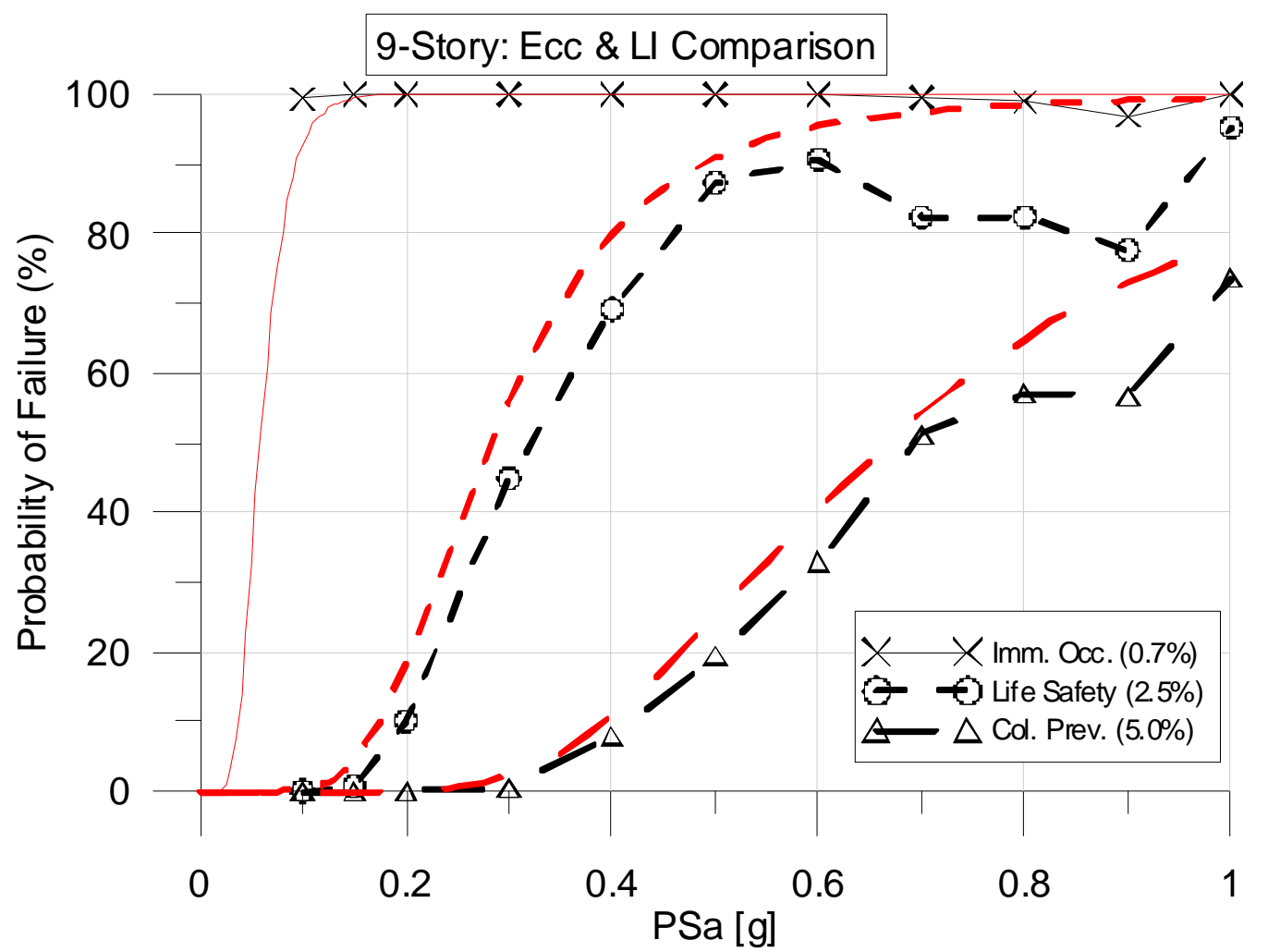

Figure E.35: Regression and non-regression fragility curve comparison for load eccentricity and intensity, 9-story 


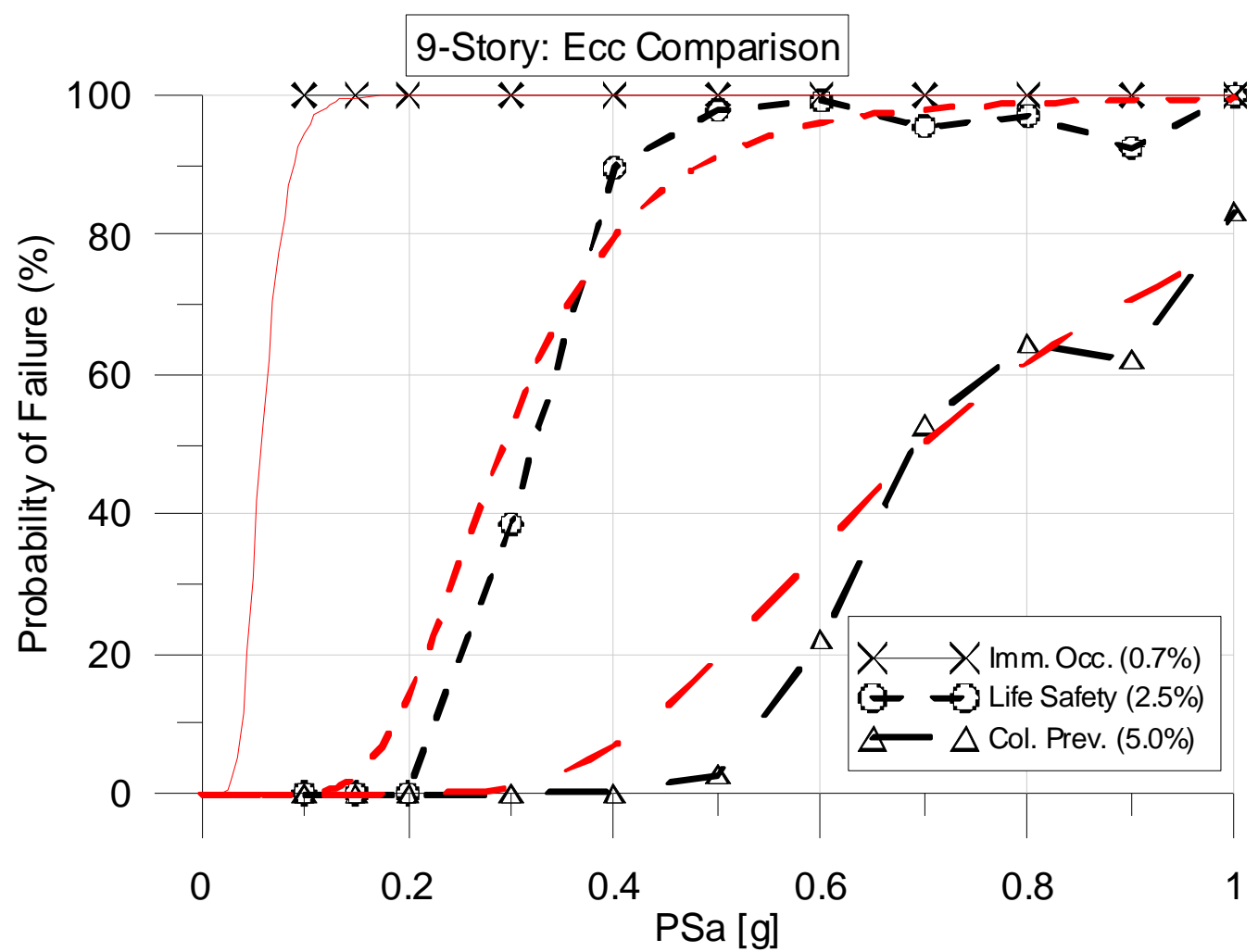

Figure E.36: Regression and non-regression fragility curve comparison for load eccentricity, 9-story

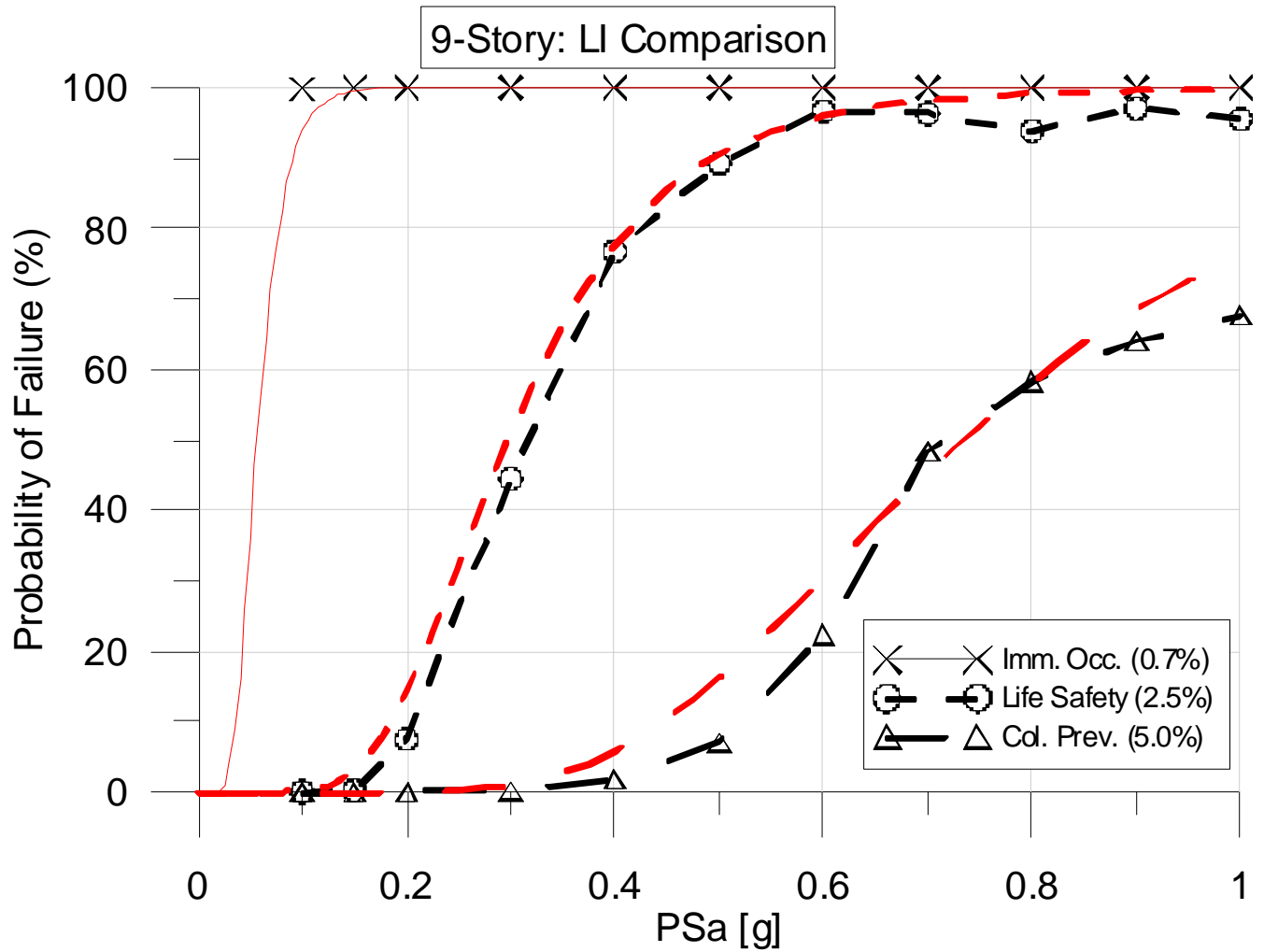

Figure E.37: Regression and non-regression fragility curve comparison for load intensity, 9-story 


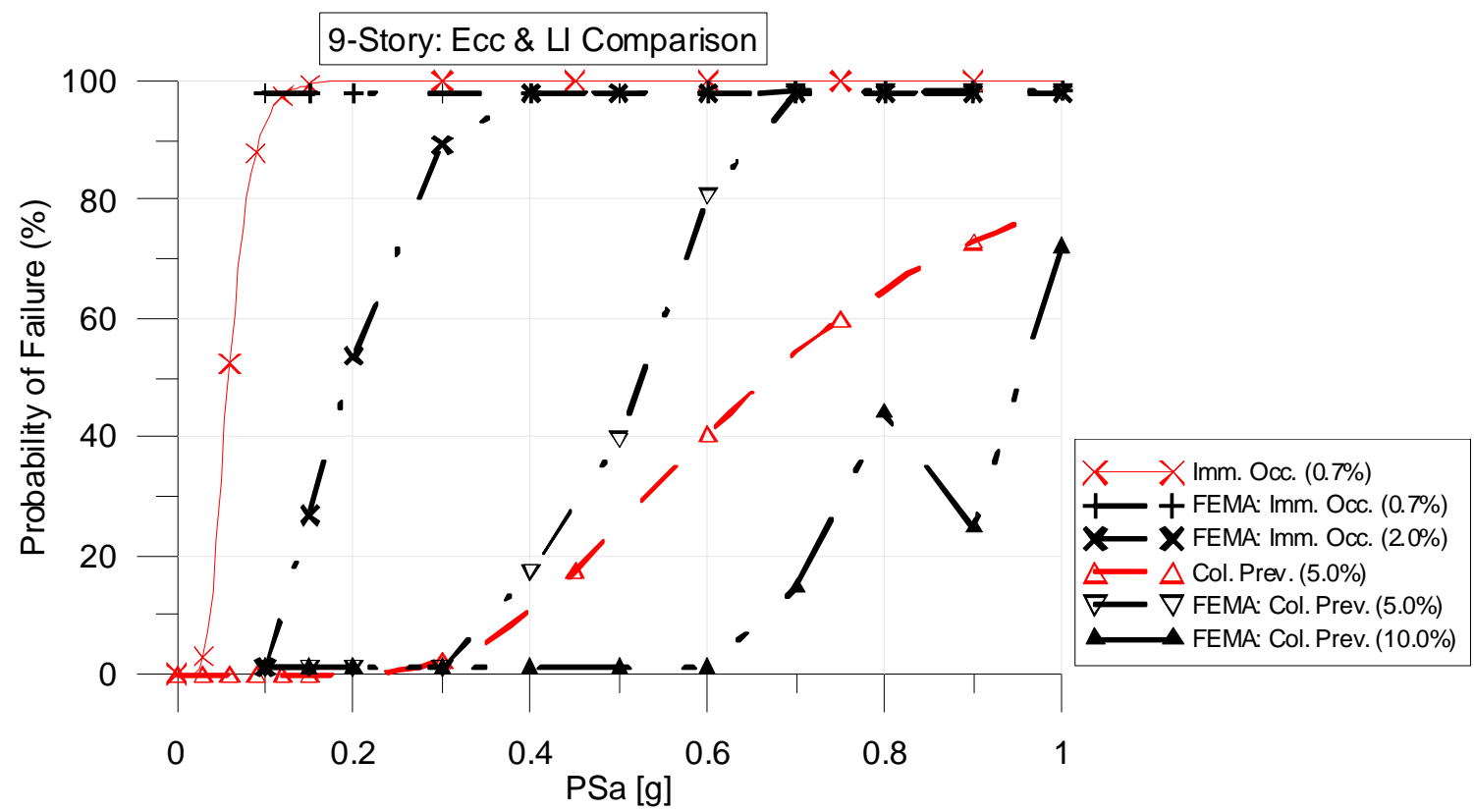

Figure E.38: Regression and FEMA comparison for load eccentricity and intensity, 9-story

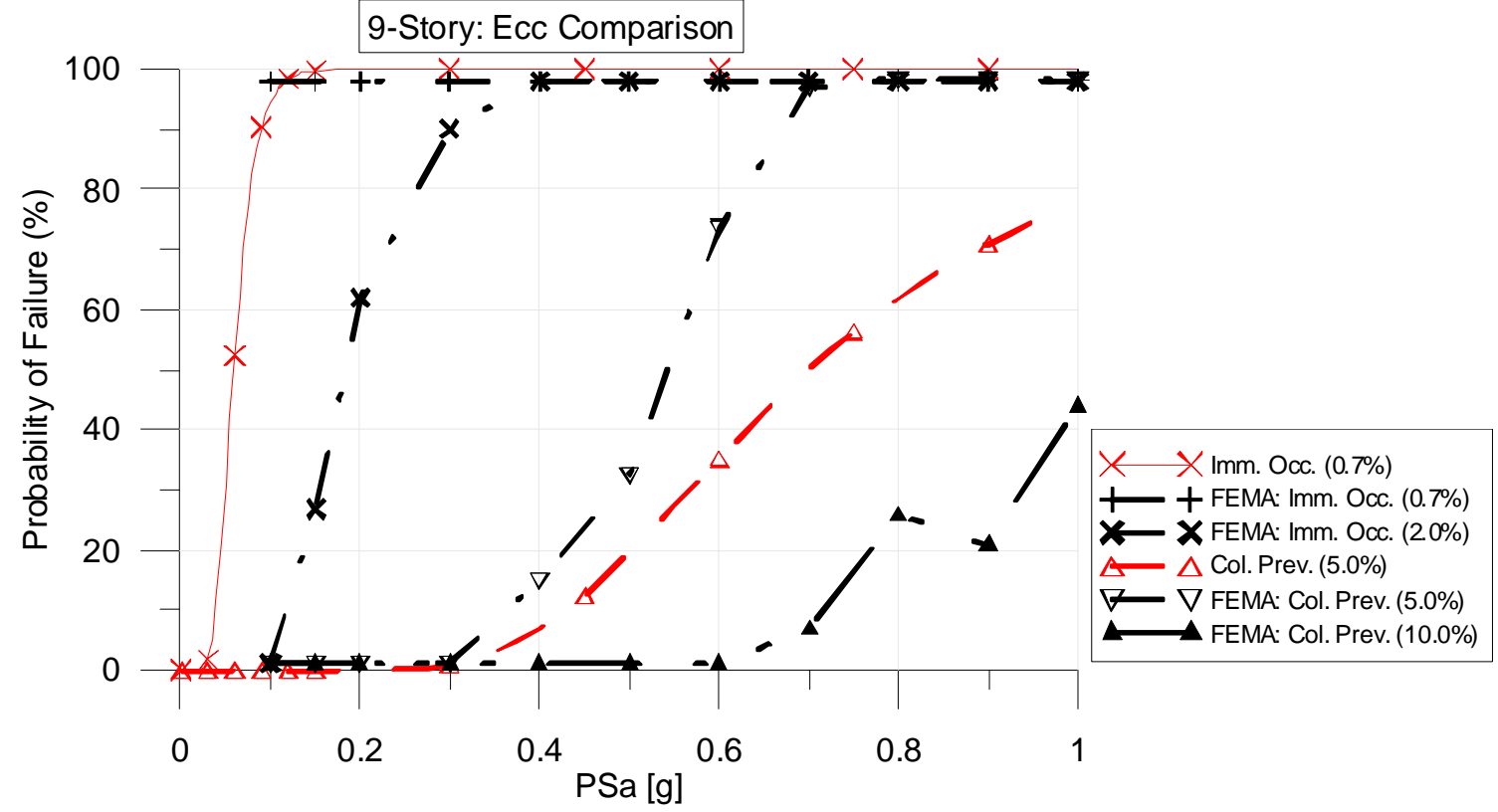

Figure E.39: Regression and FEMA comparison for load eccentricity, 9-story 


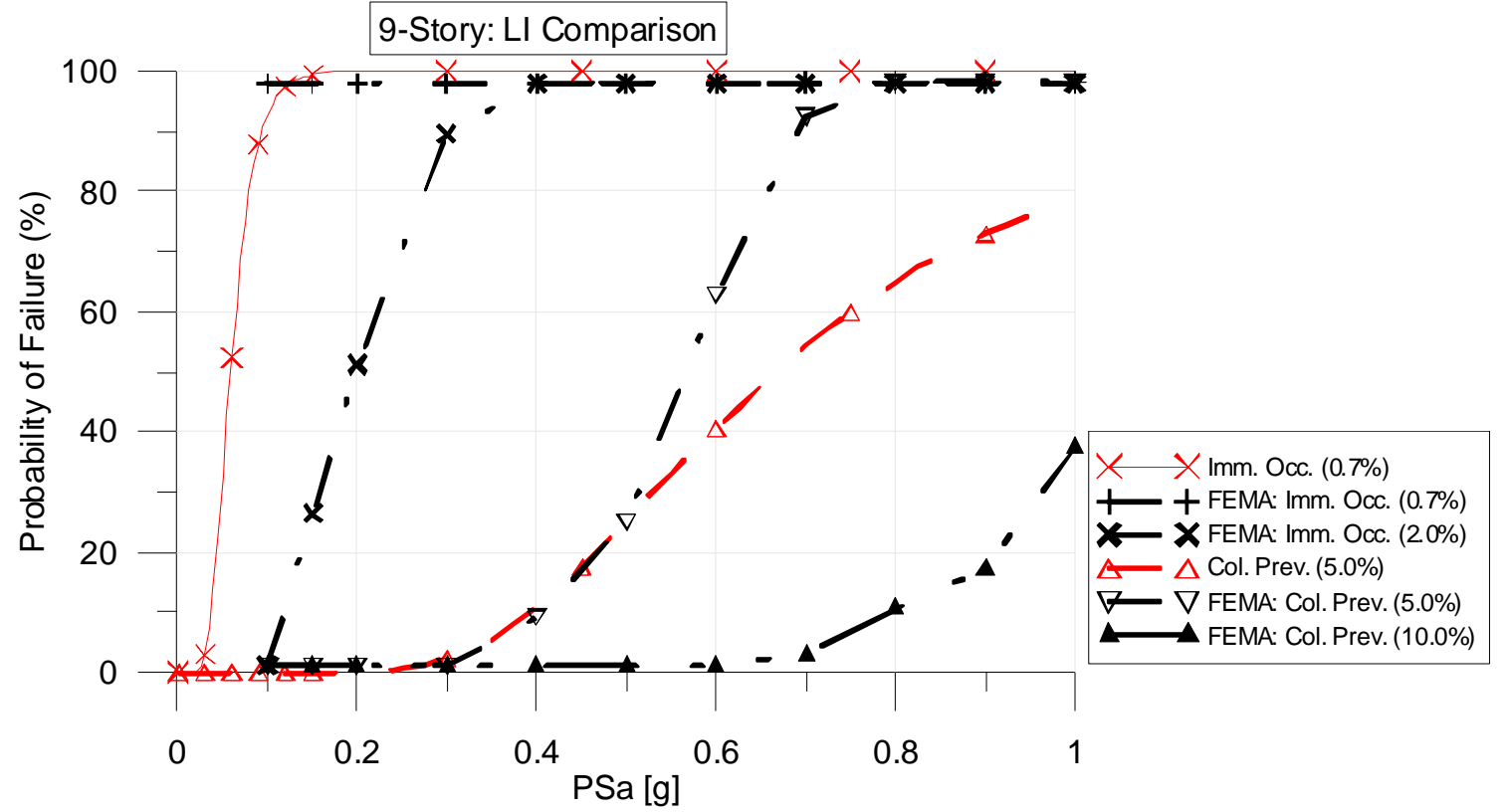

Figure E.40: Regression and FEMA comparison for load intensity, 9-story 


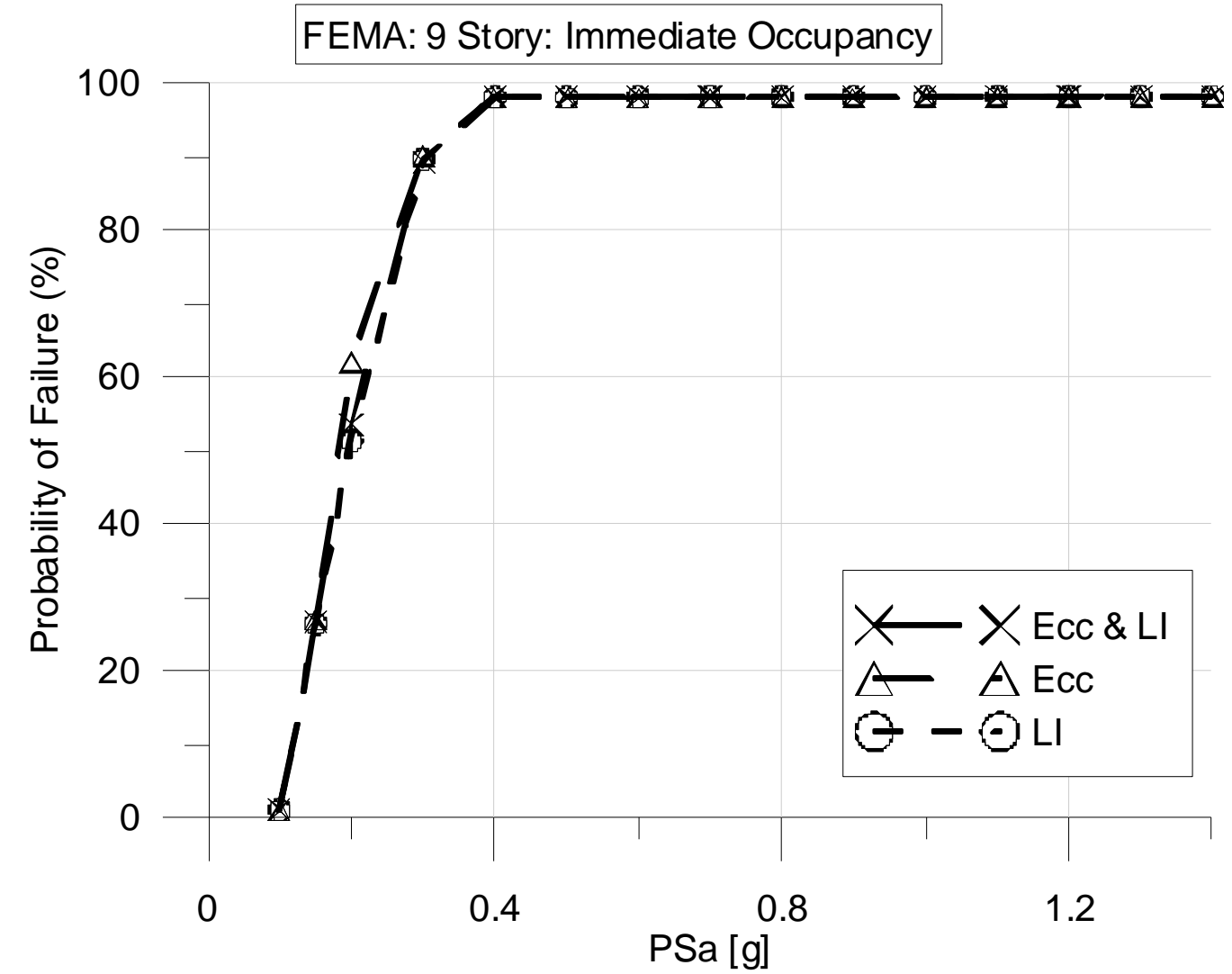

Figure E.41: FEMA 350 fragility curves for immediate occupancy comparison, 9-story

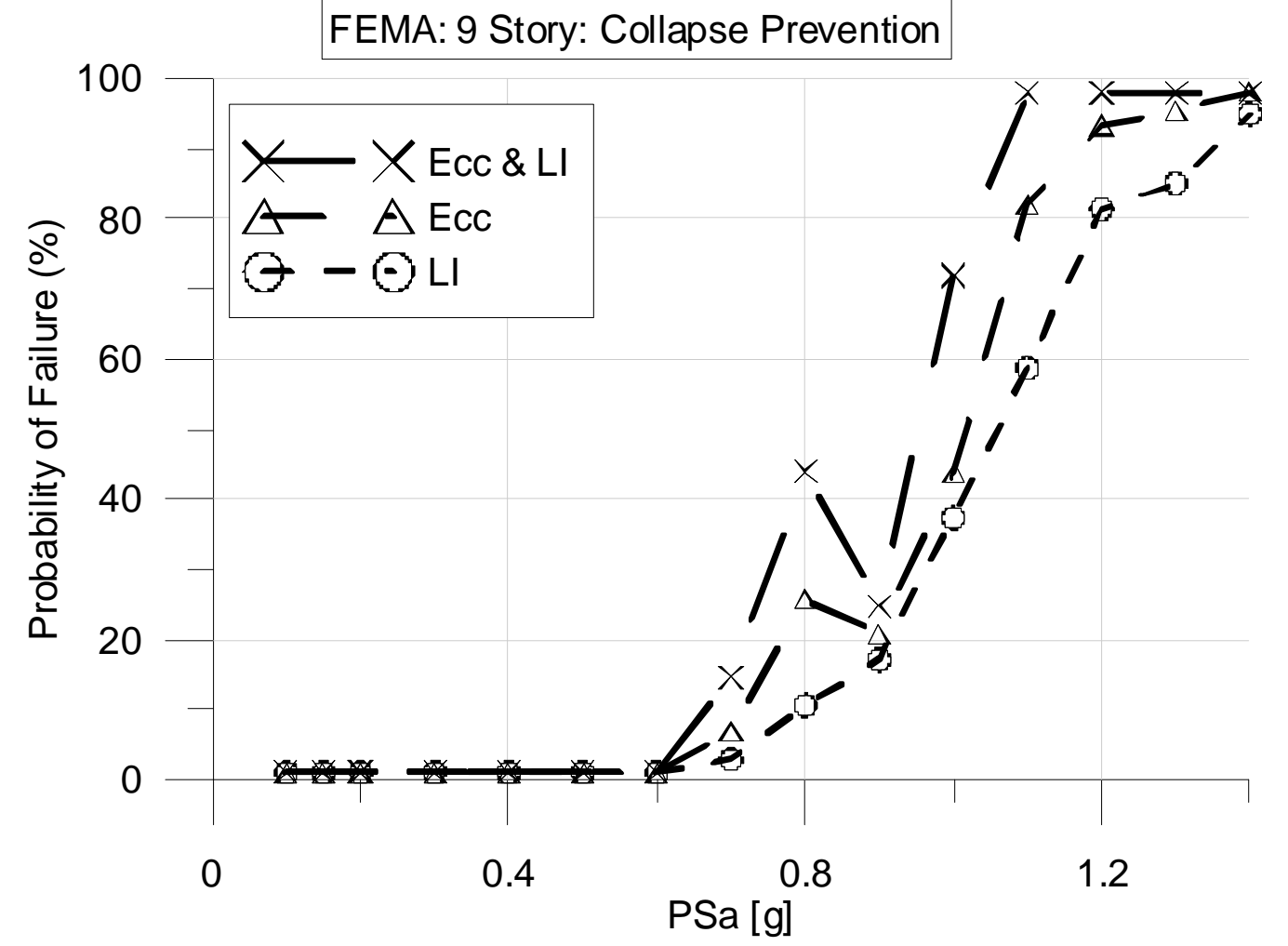

Figure E.42: FEMA 350 fragility curves for collapse prevention comparison, 9-story 


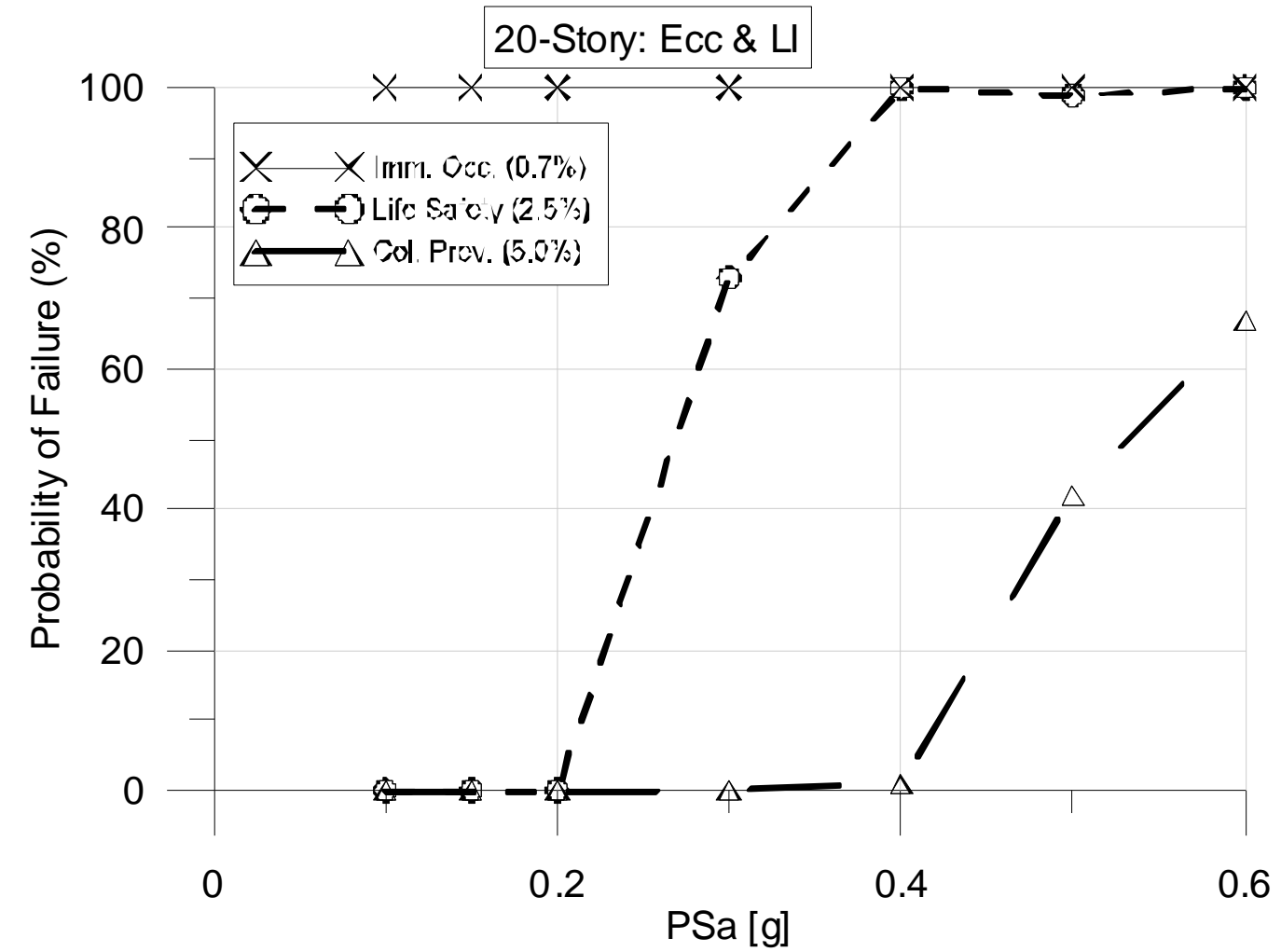

Figure E.43: Non-regression fragility curves for load eccentricity and intensity, 20-story

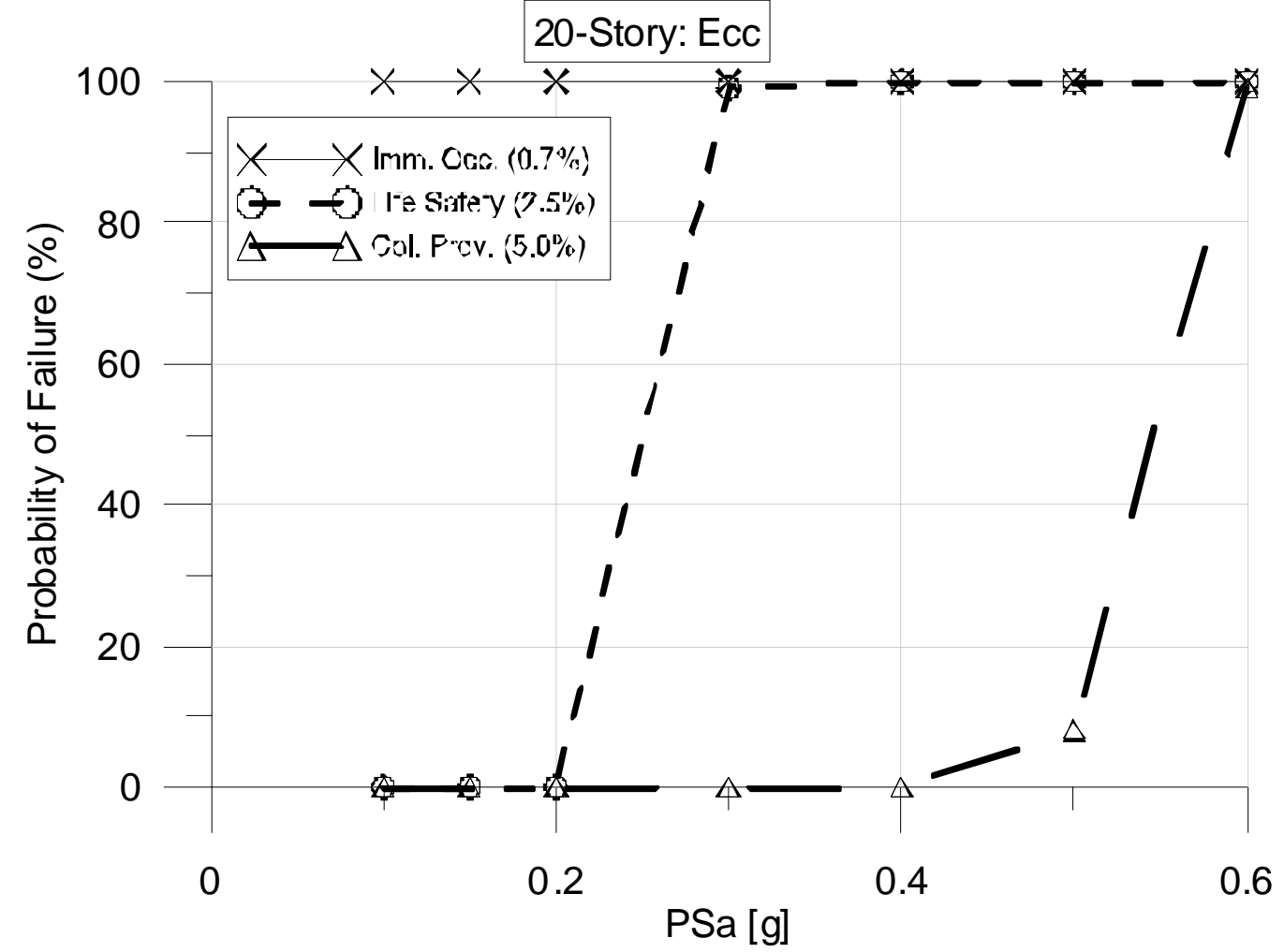

Figure E.44: Non-regression fragility curves for load eccentricity, 20-story 


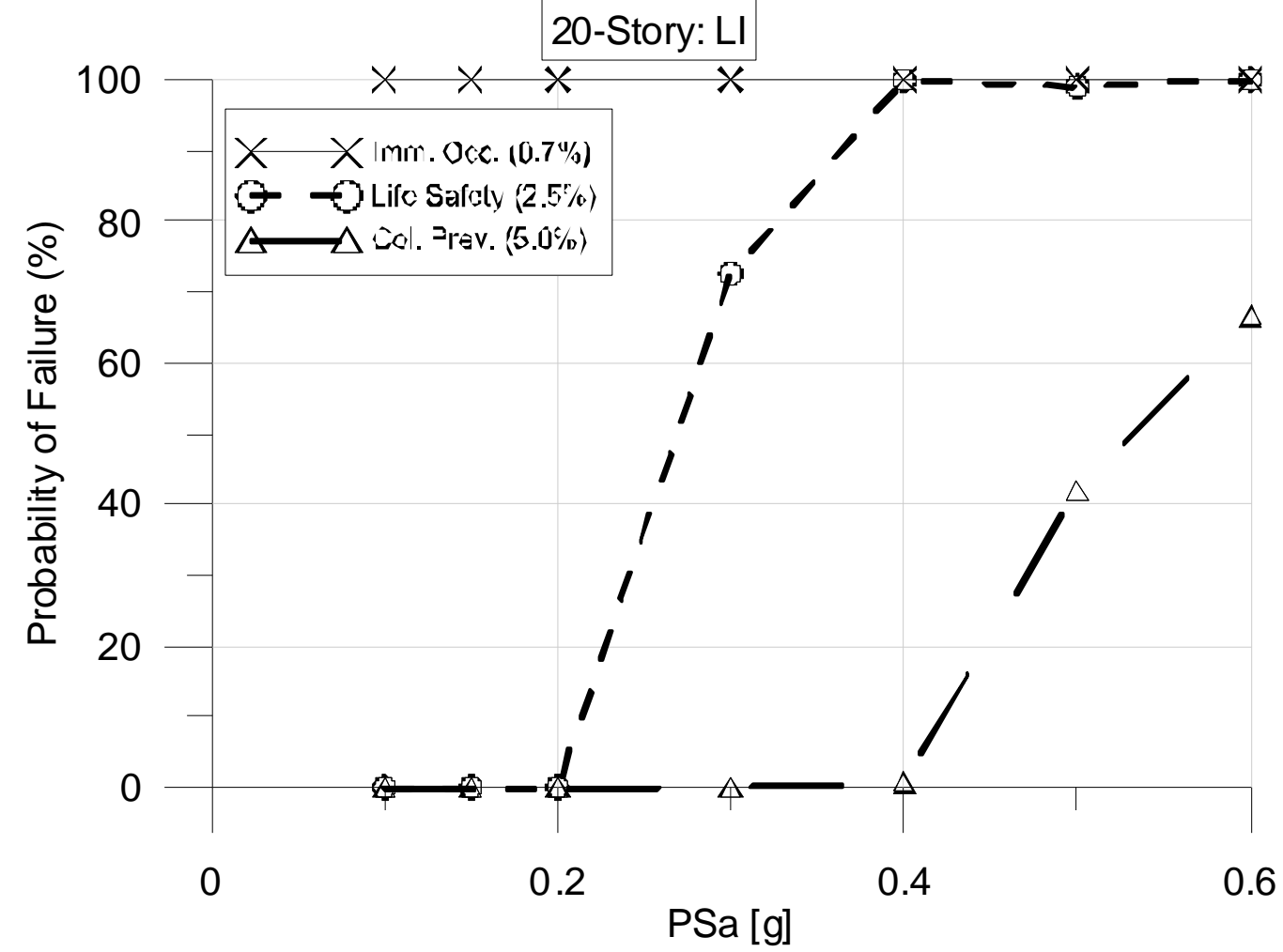

Figure E.45: Non-regression fragility curves for load intensity, 20-story

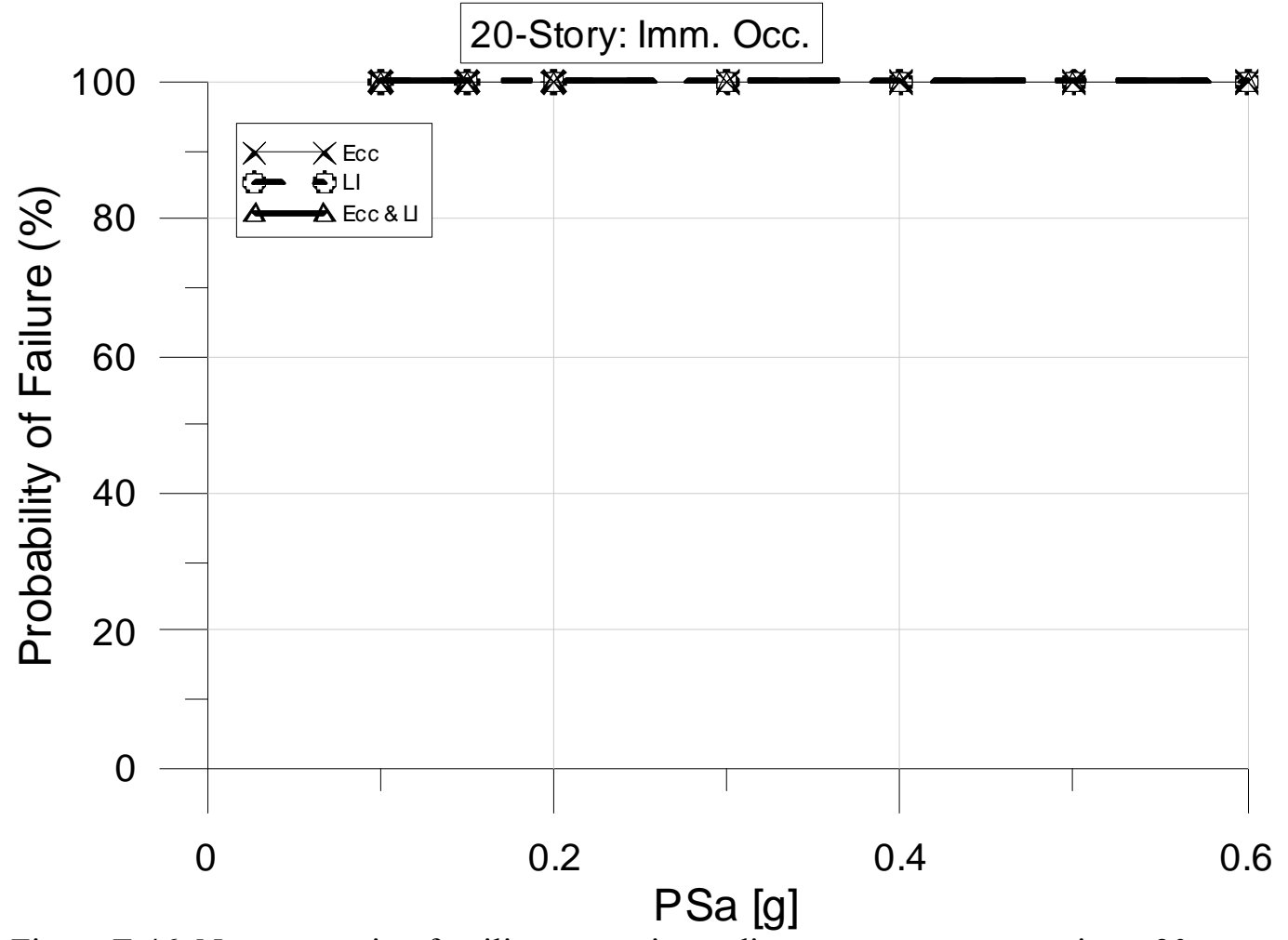

Figure E.46: Non-regression fragility curves immediate occupancy comparison, 20-story 


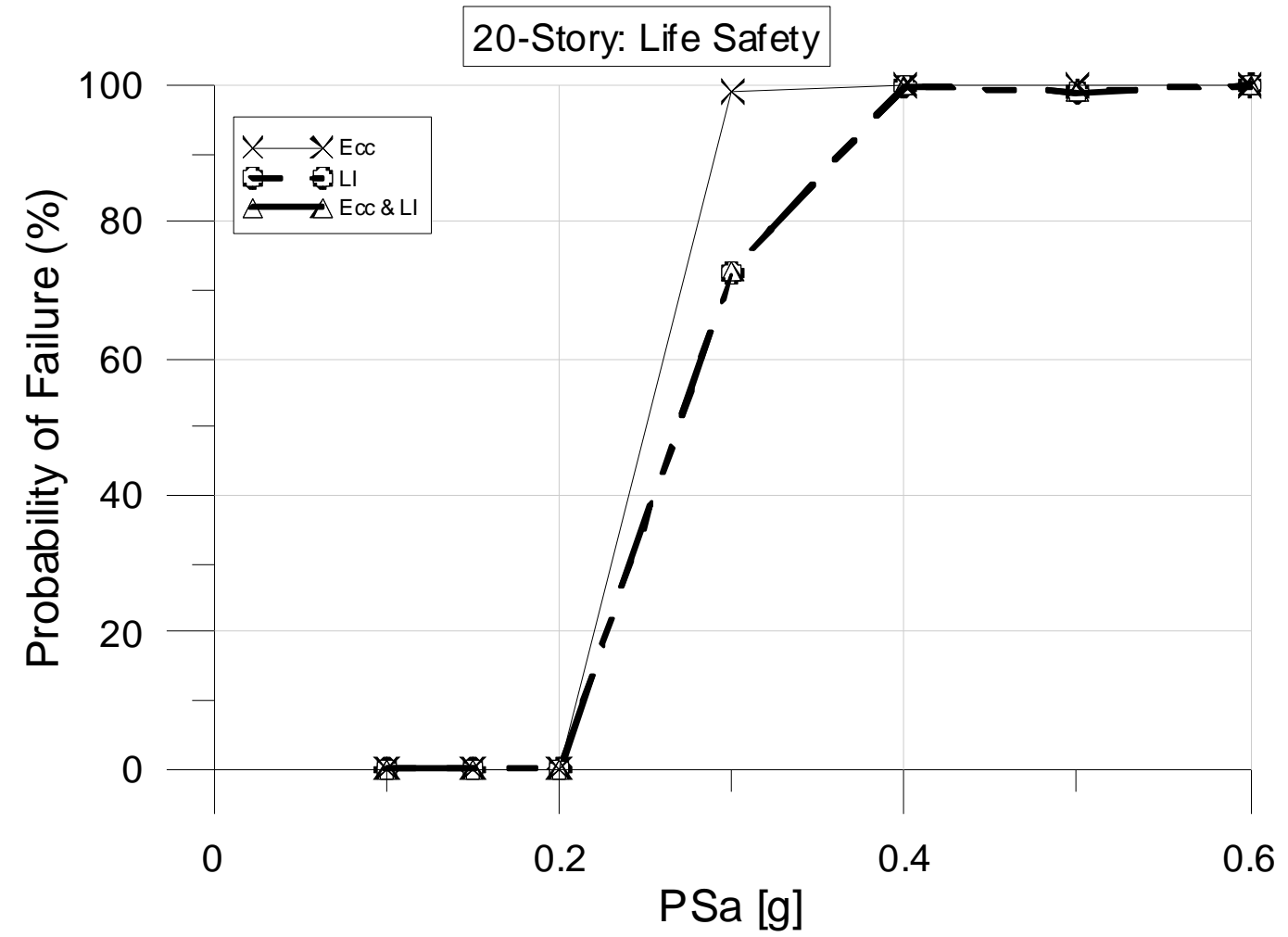

Figure E.47: Non-regression fragility curves life safety comparison, 20-story

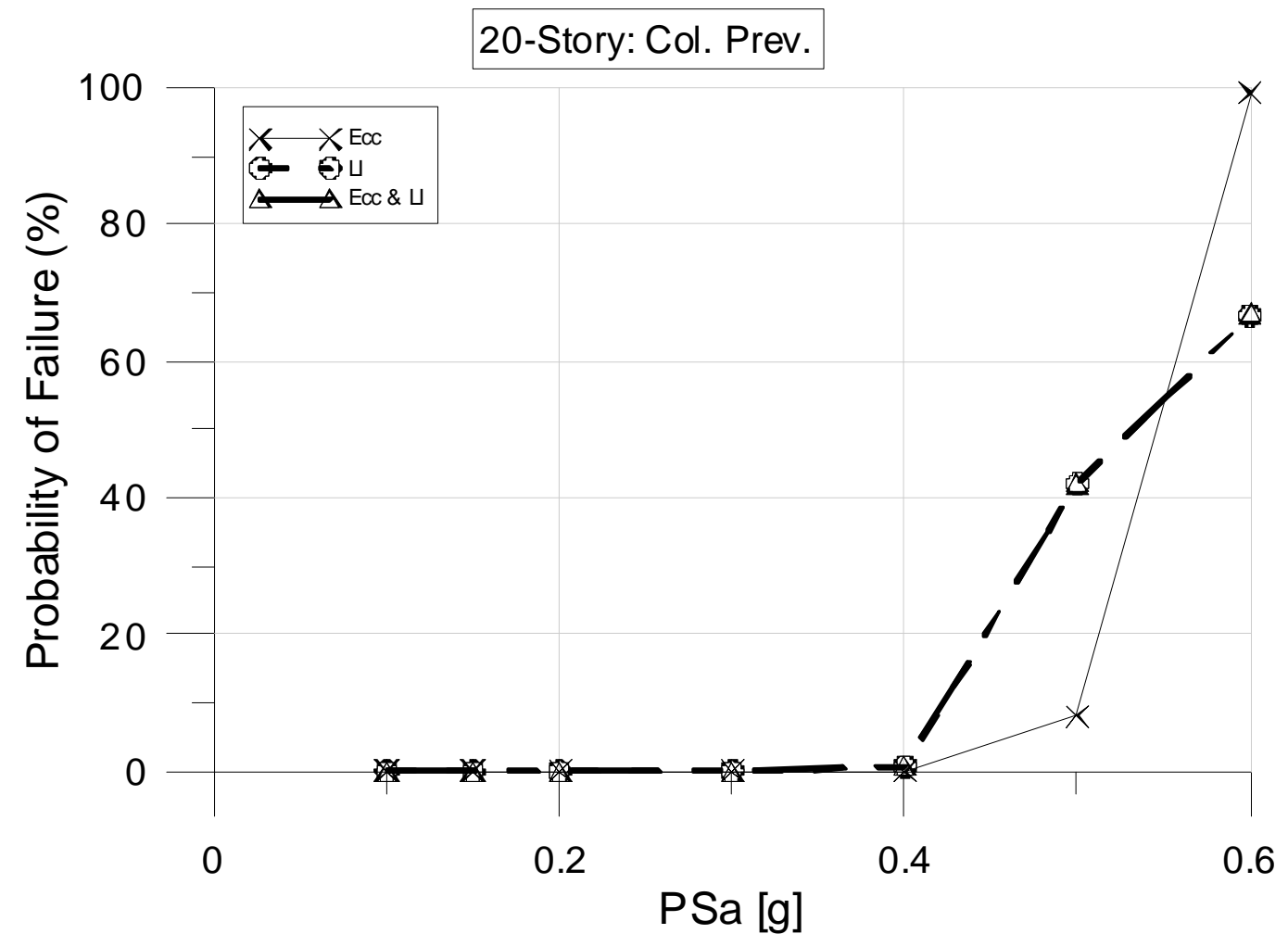

Figure E.48: Non-regression fragility curves collapse prevention comparison, 20-story 


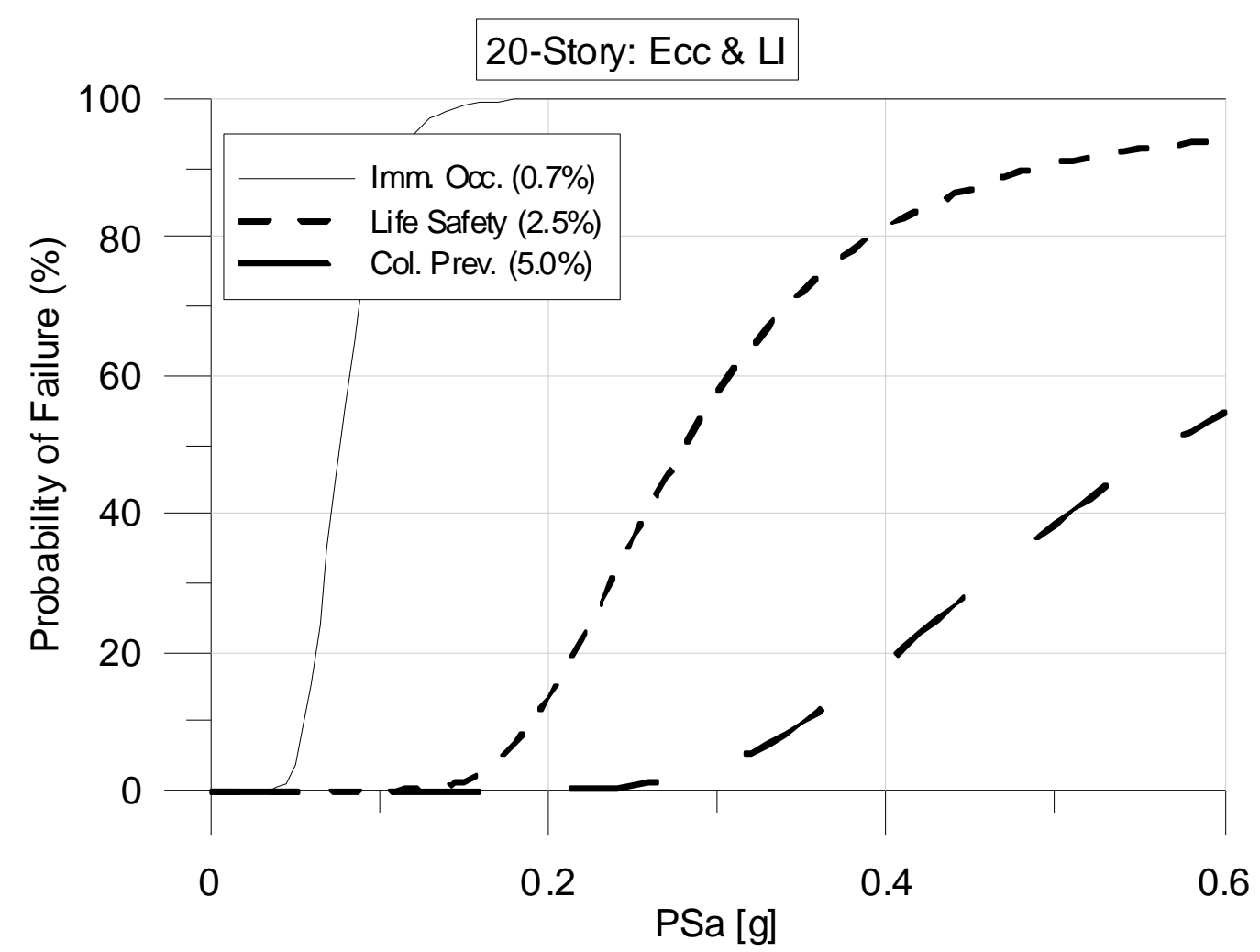

Figure E.49: Regression fragility curves for load eccentricity and intensity, 20-story

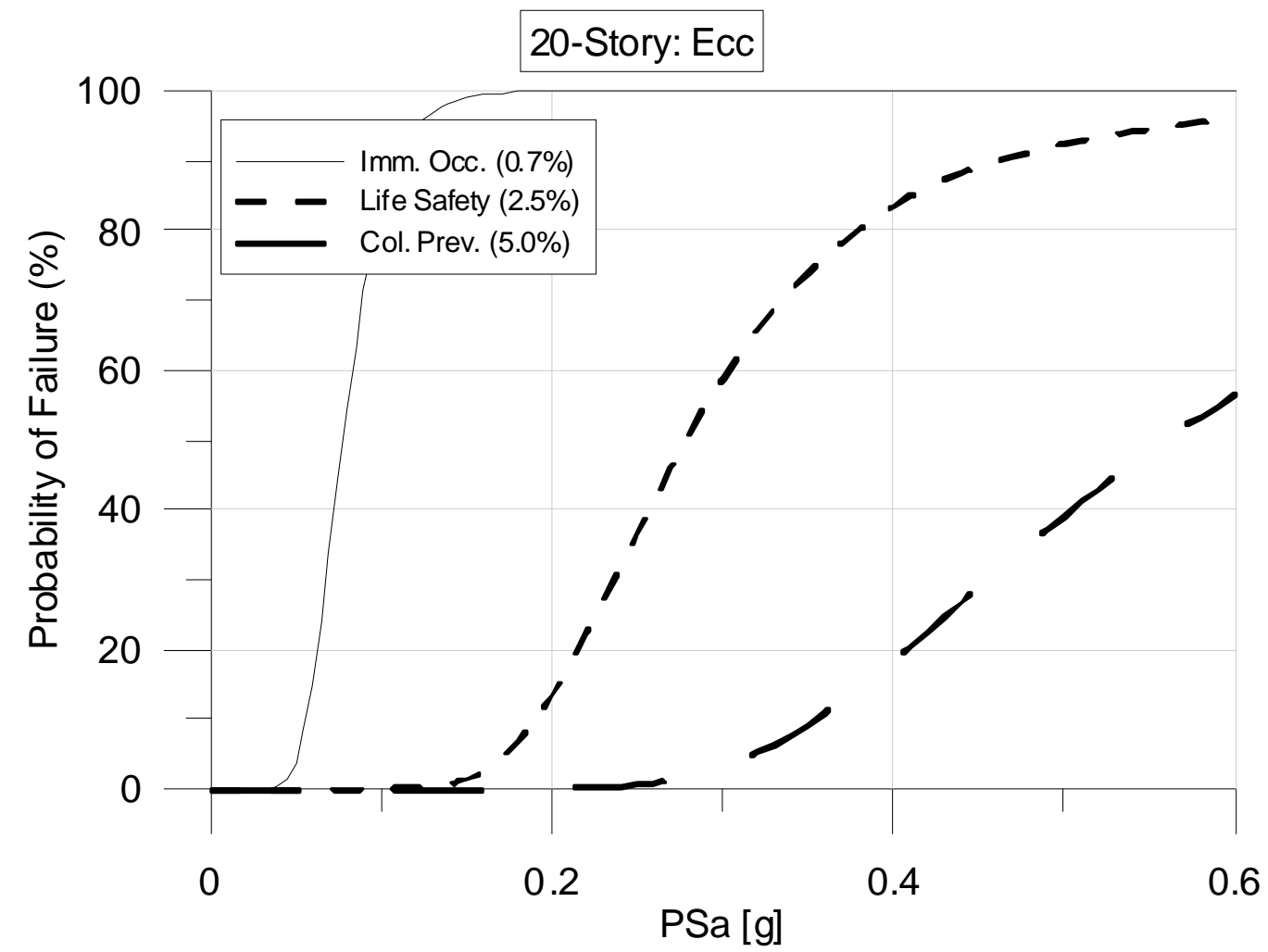

Figure E.50: Regression fragility curves for load eccentricity, 20-story 


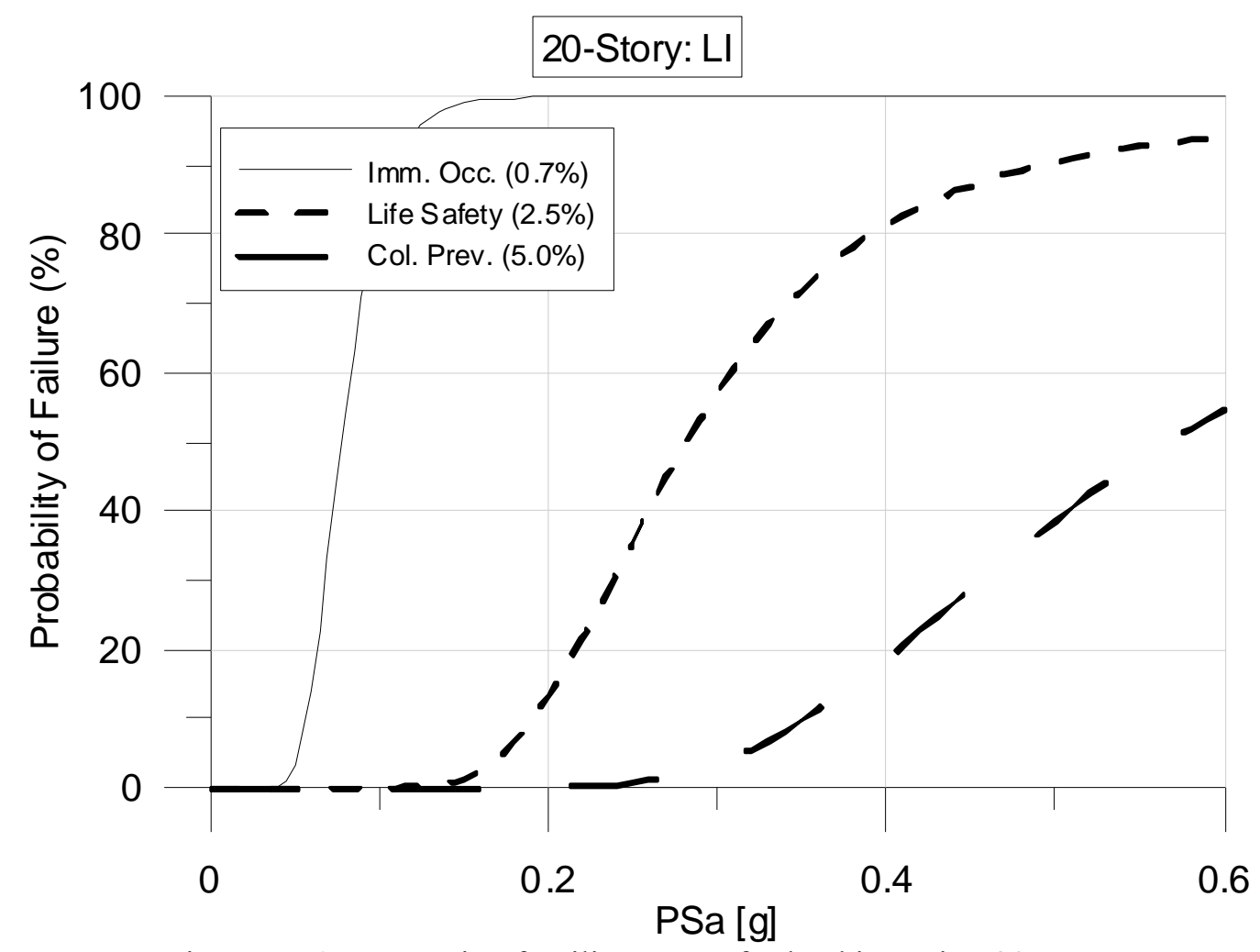

Figure E.51: Regression fragility curves for load intensity, 20-story

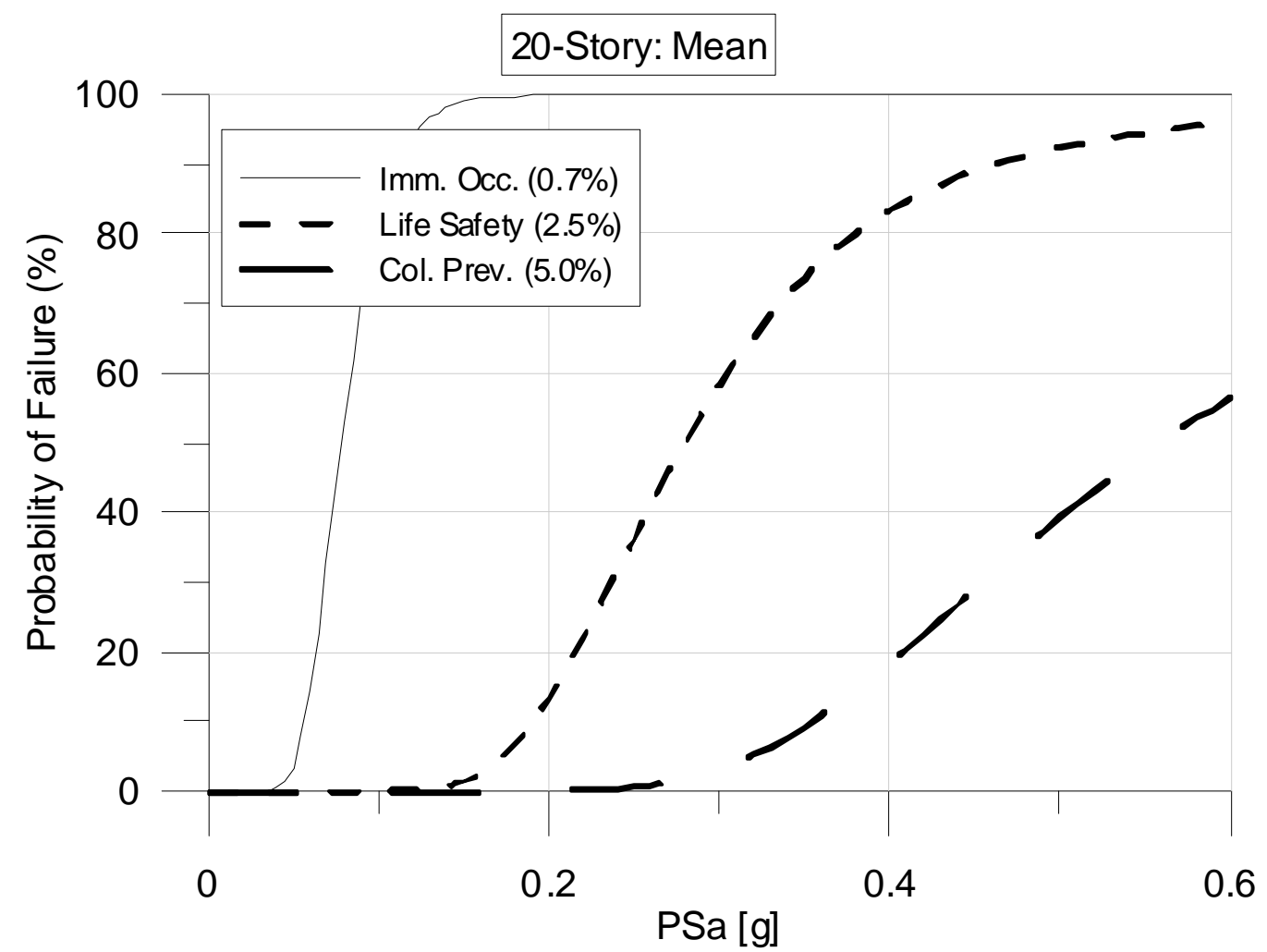

Figure E.52: Regression fragility curves for mean values of eccentricity and intensity, 20-story 


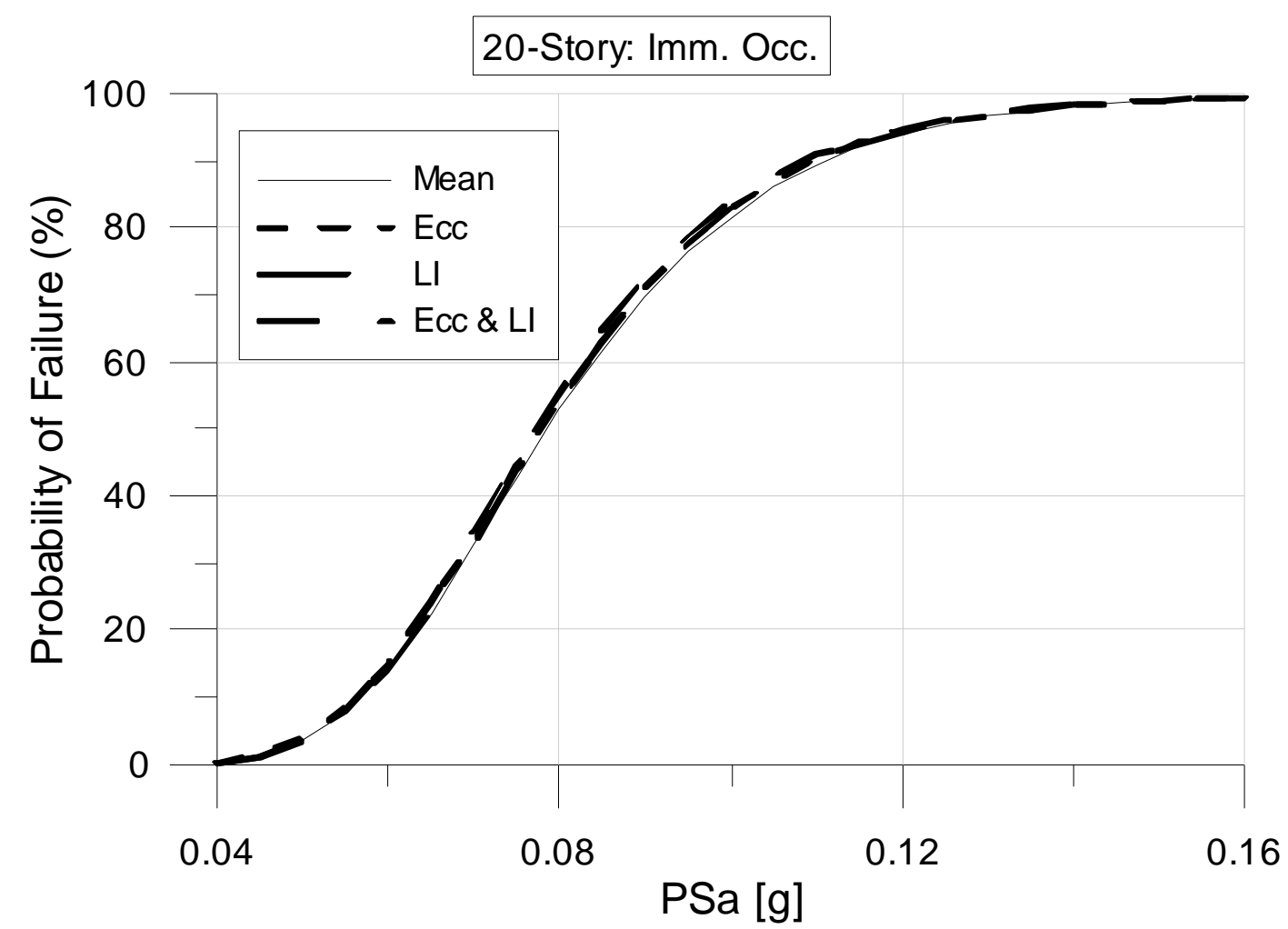

Figure E.53: Regression fragility curves for immediate occupancy comparison, 20-story

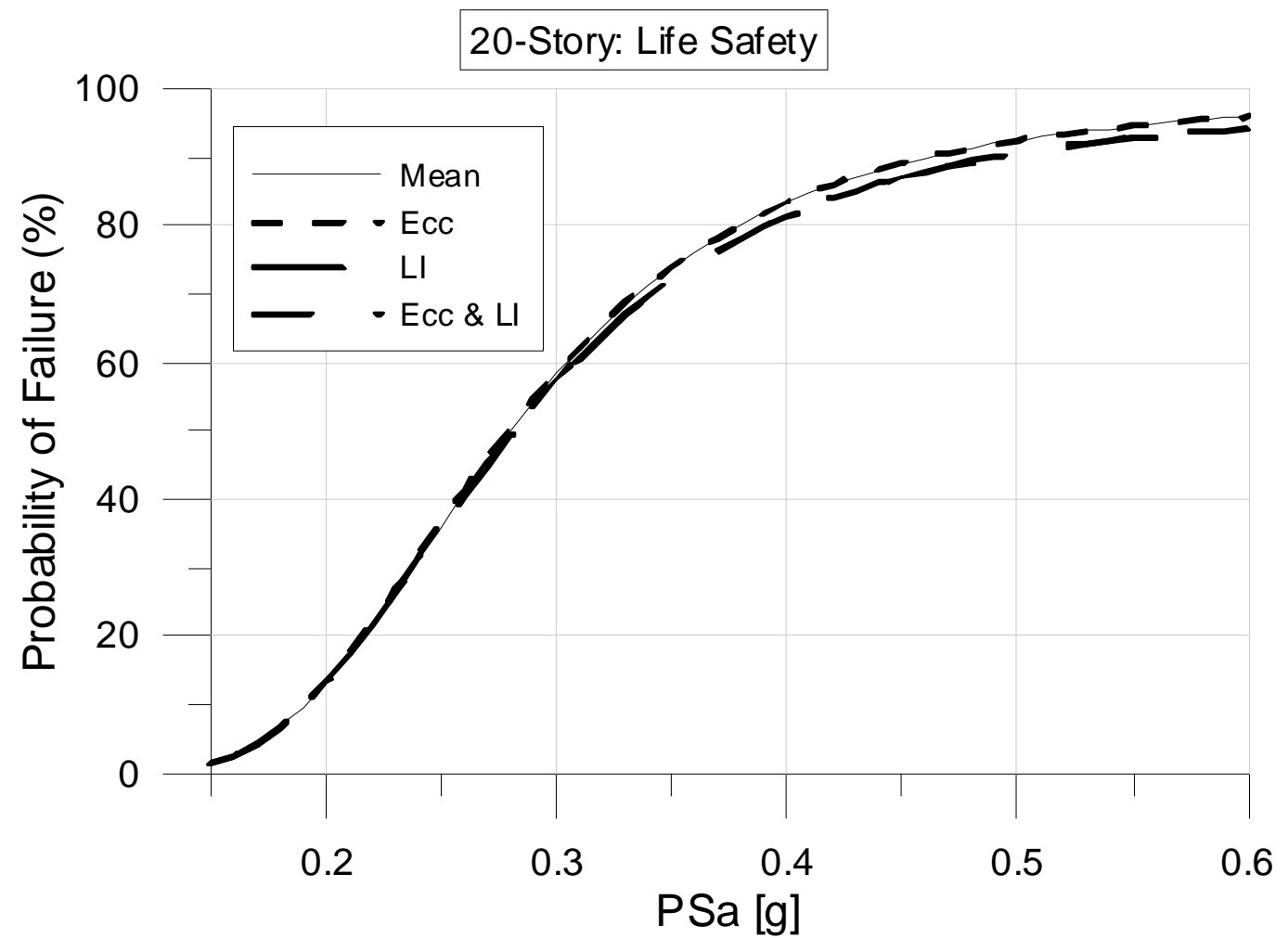

Figure E.54: Regression fragility curves for life safety comparison, 20-story 


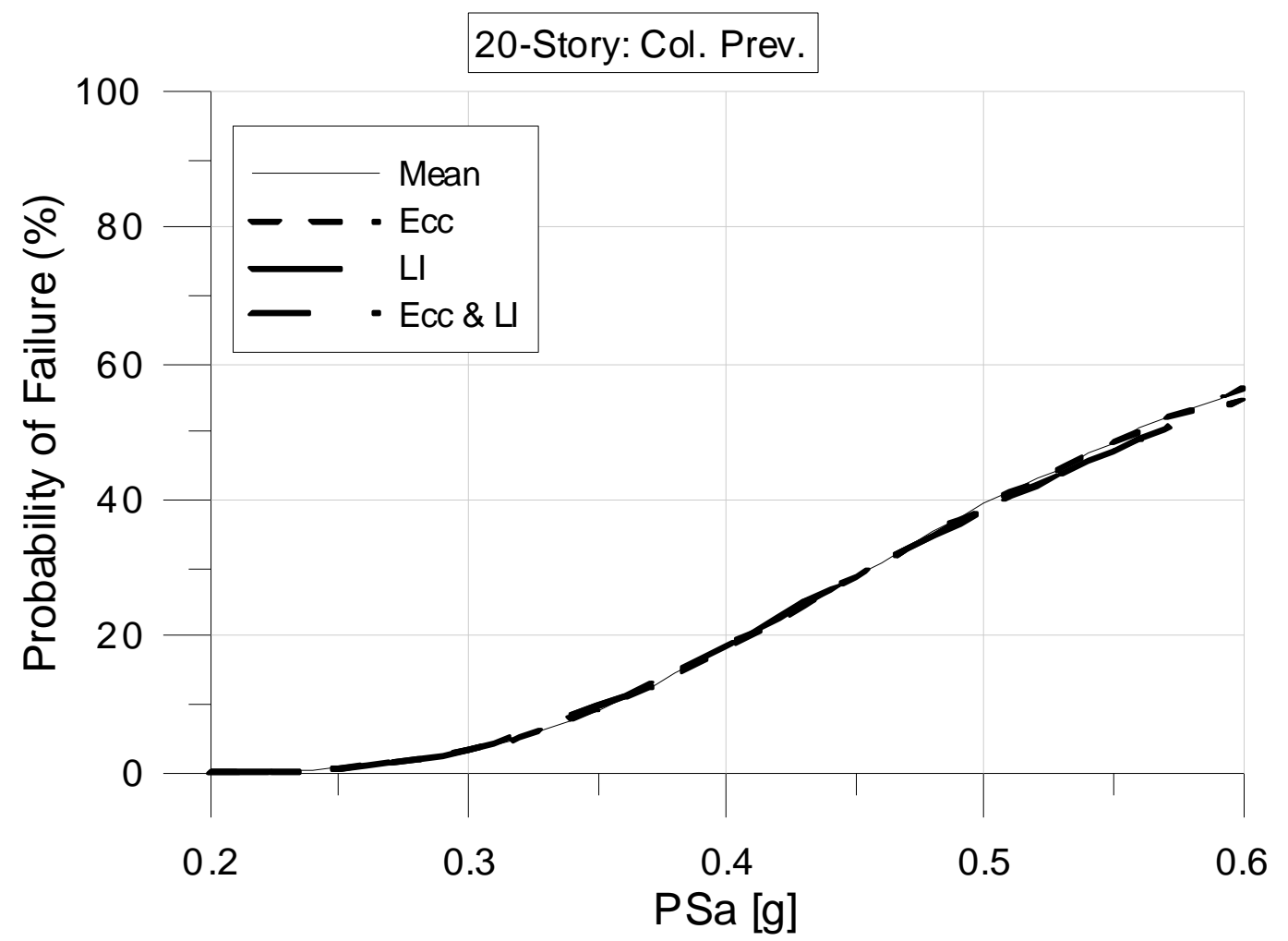

Figure E.55: Regression fragility curves for collapse prevention comparison, 20-story

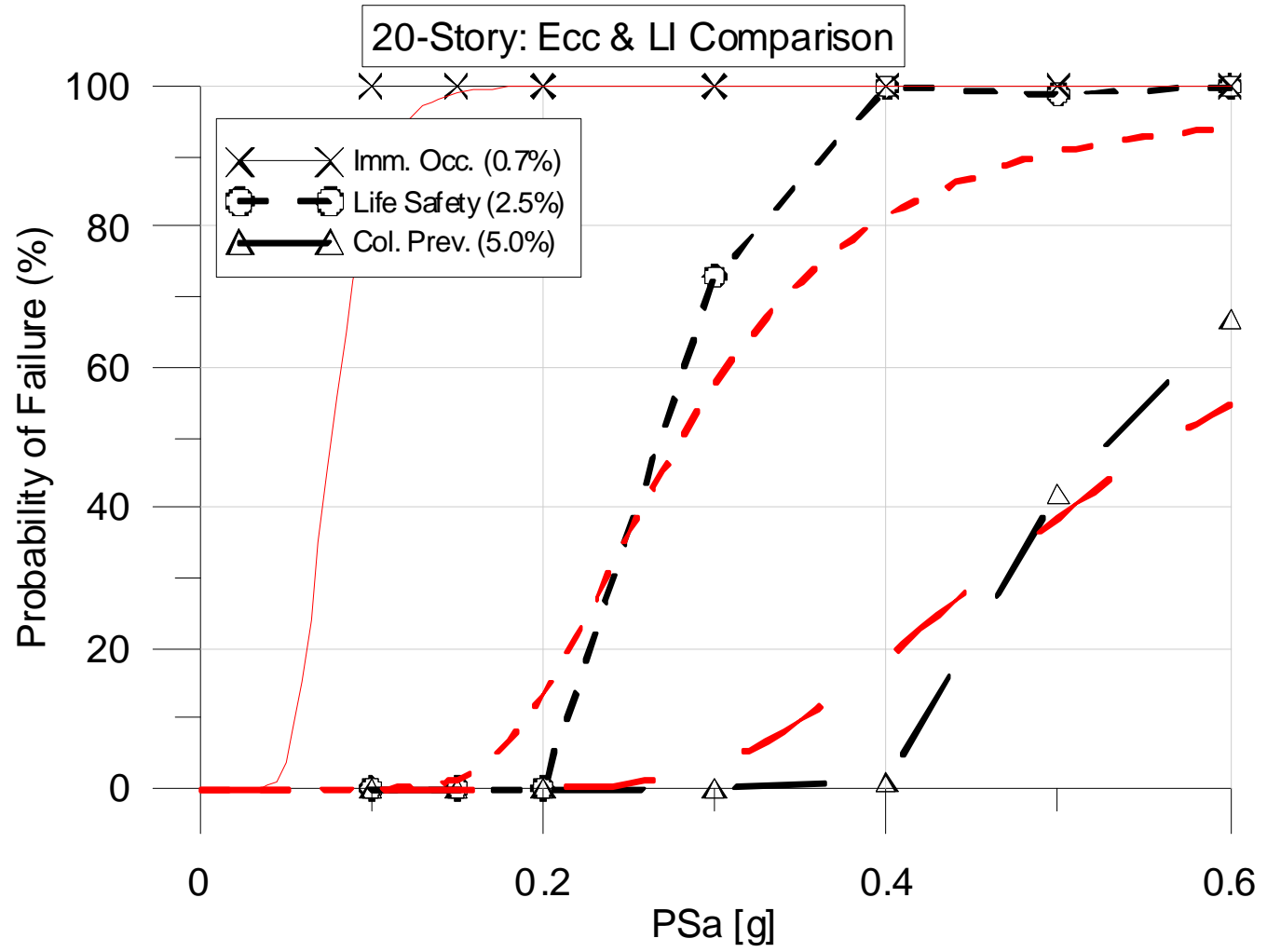

Figure E.56: Regression and non-regression fragility curve comparison for load eccentricity and intensity, 20-story 


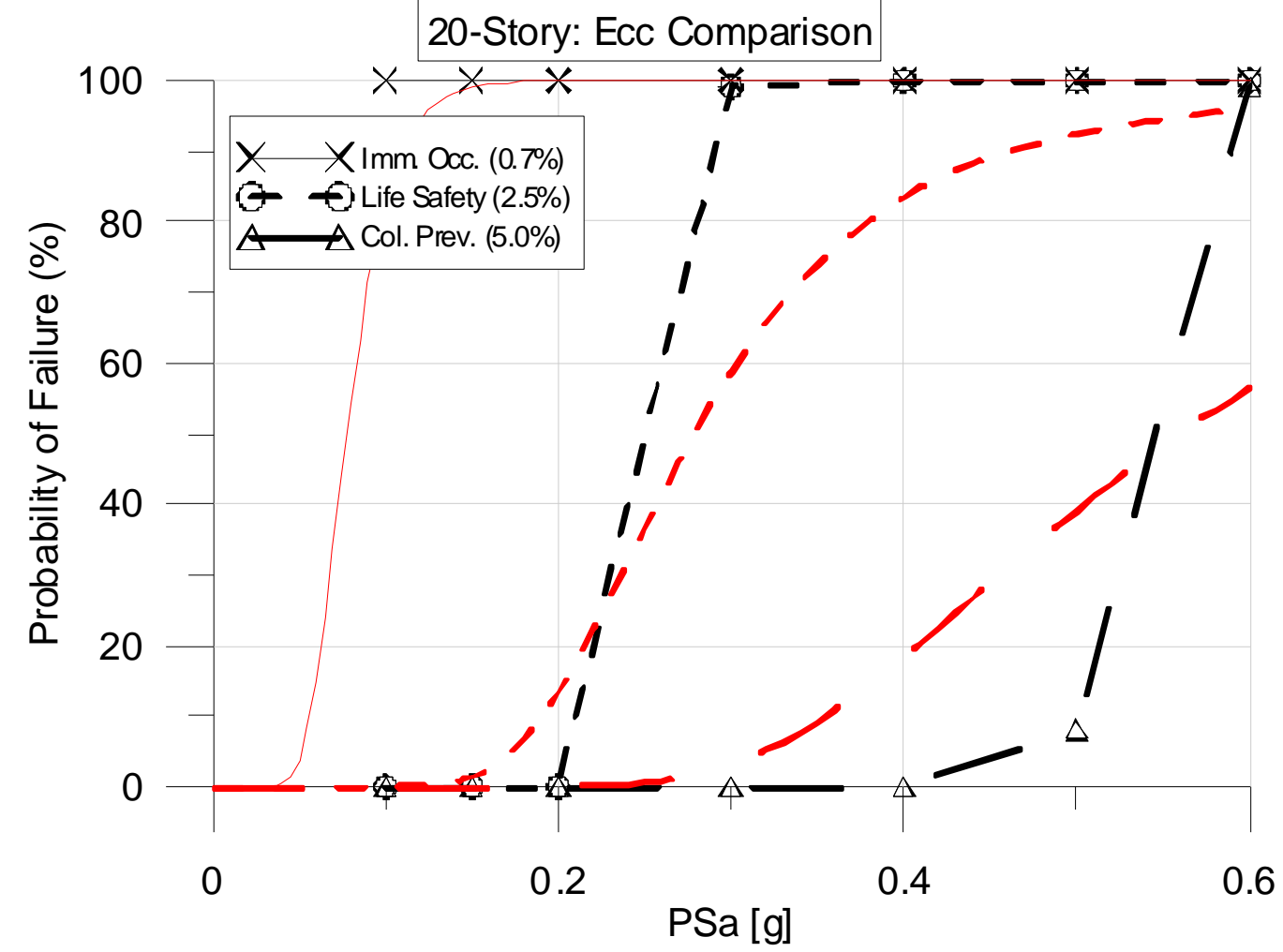

Figure E.57: Regression and non-regression fragility curve comparison for load eccentricity, 20-story

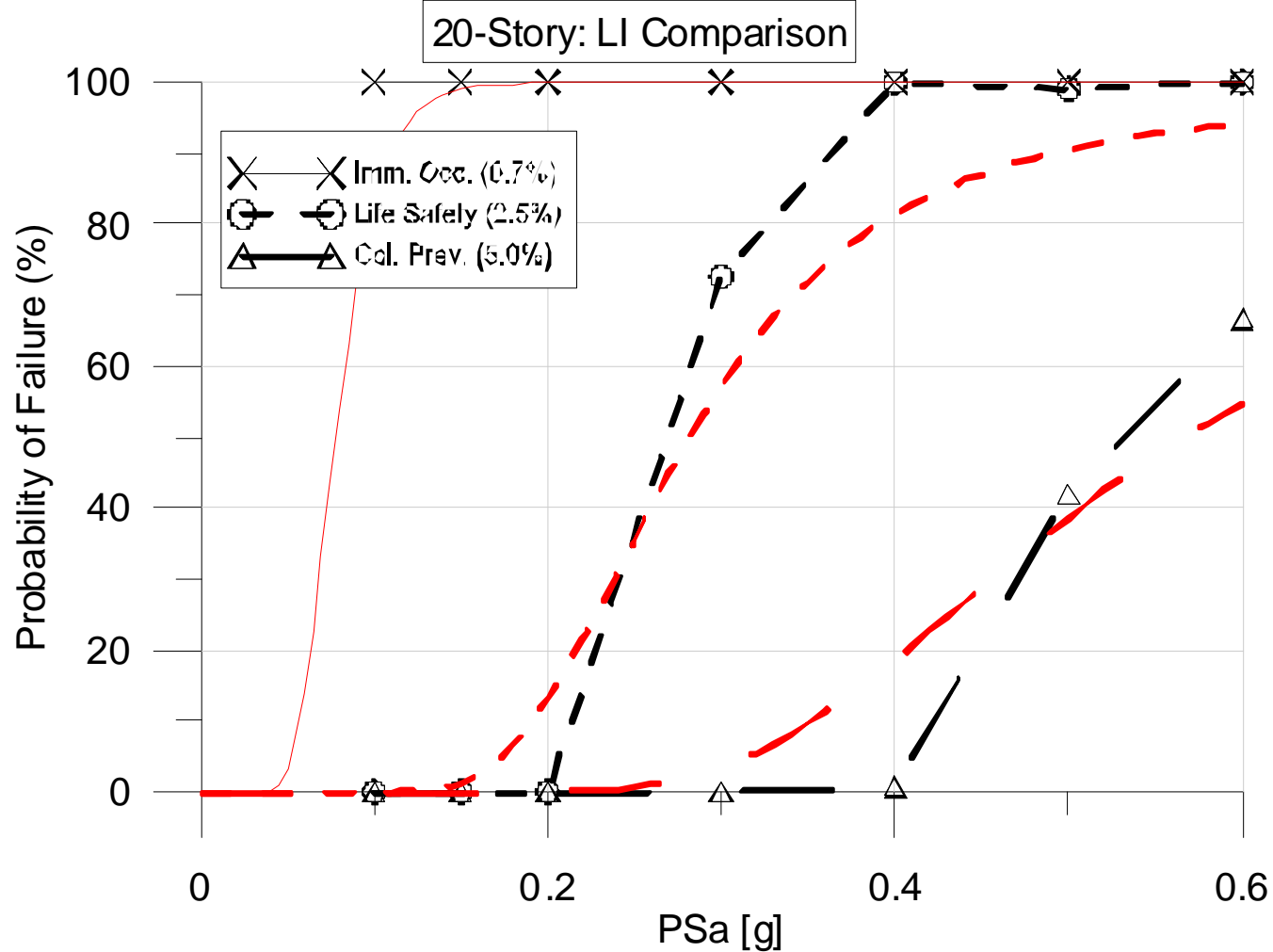

Figure E.58: Regression and non-regression fragility curve comparison for load intensity, 20-story 


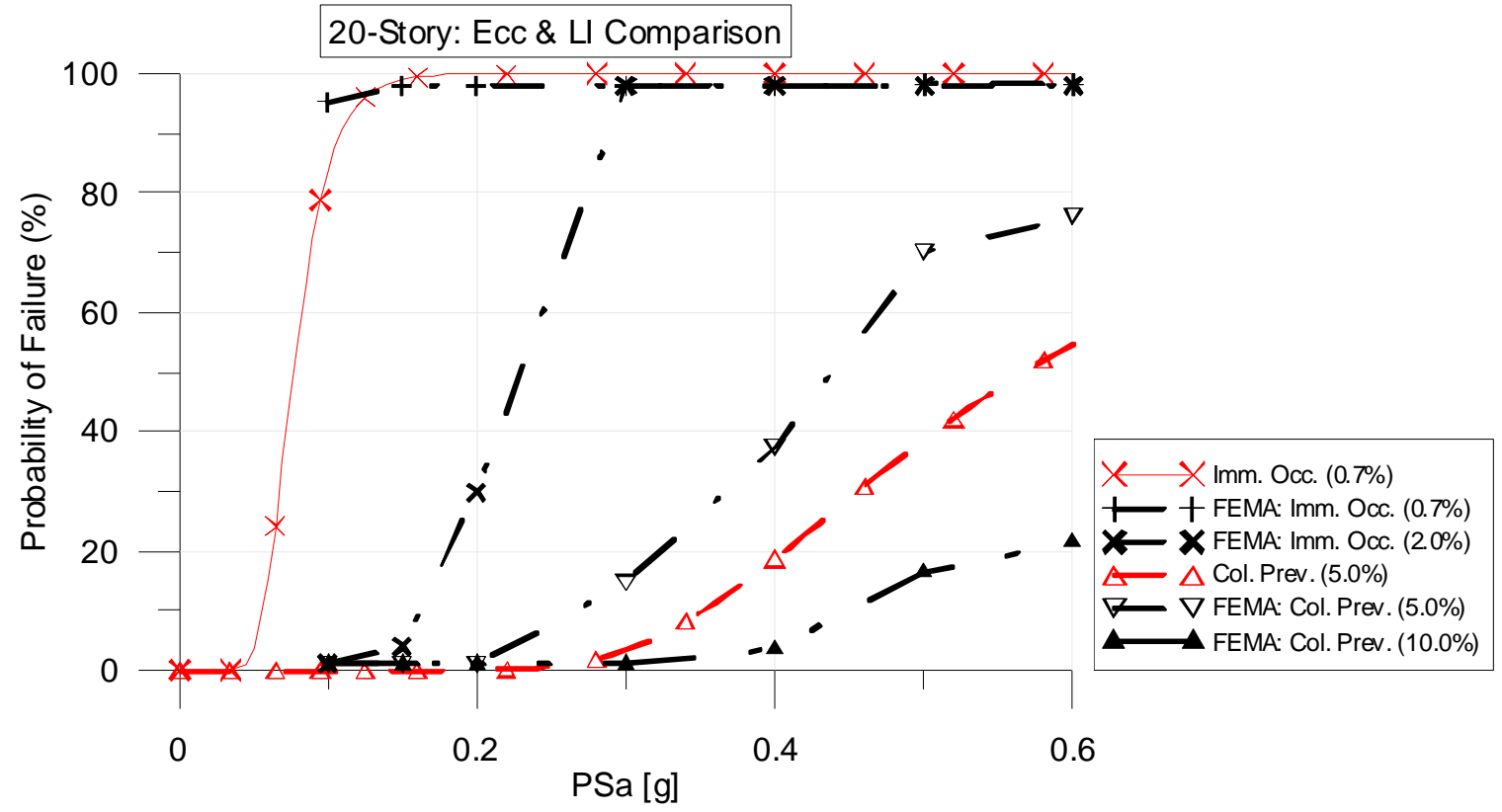

Figure E.59: Regression and FEMA comparison for load eccentricity and intensity, 20-story

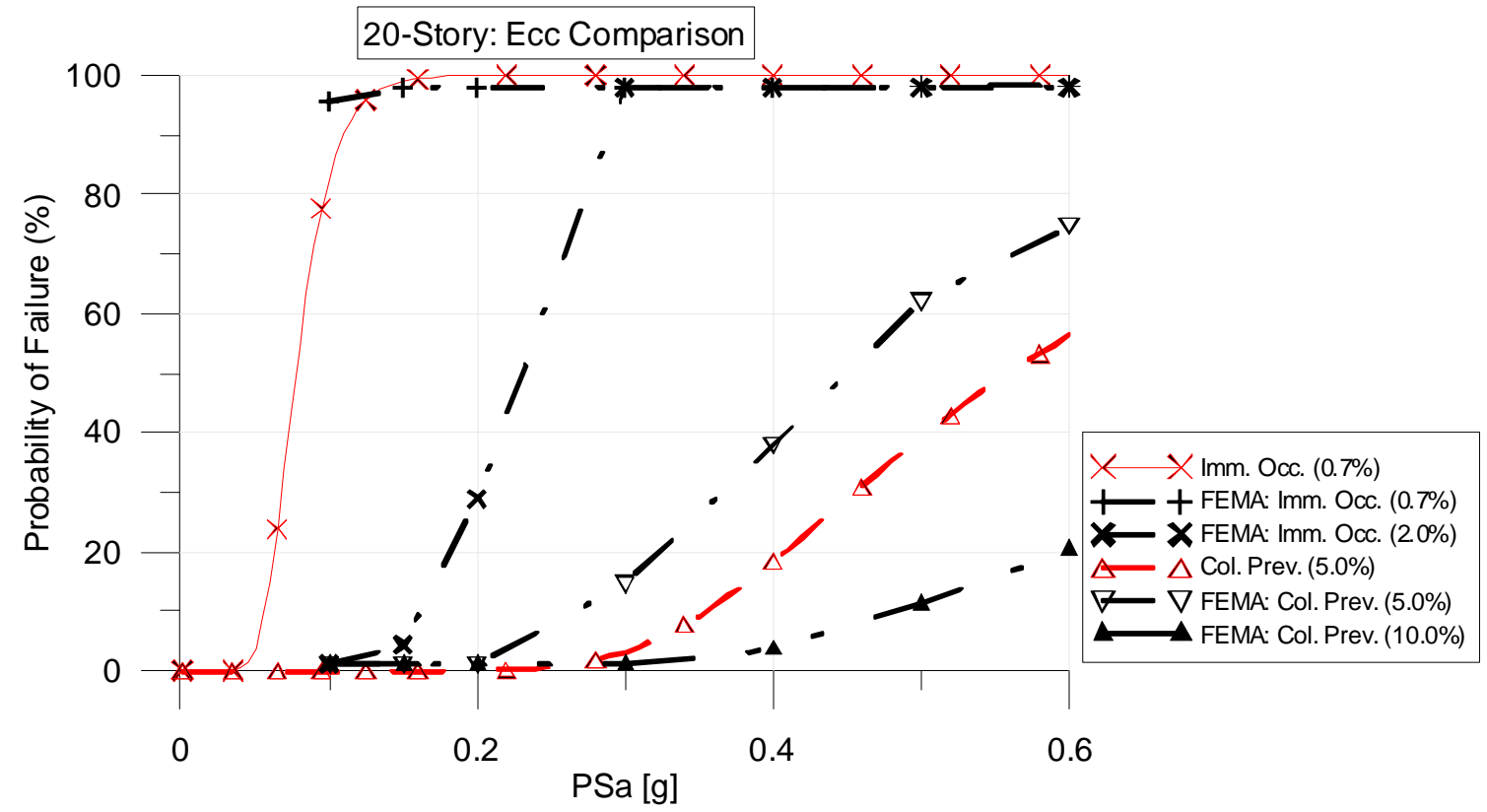

Figure E.60 Regression and FEMA comparison for load eccentricity, 20-story 


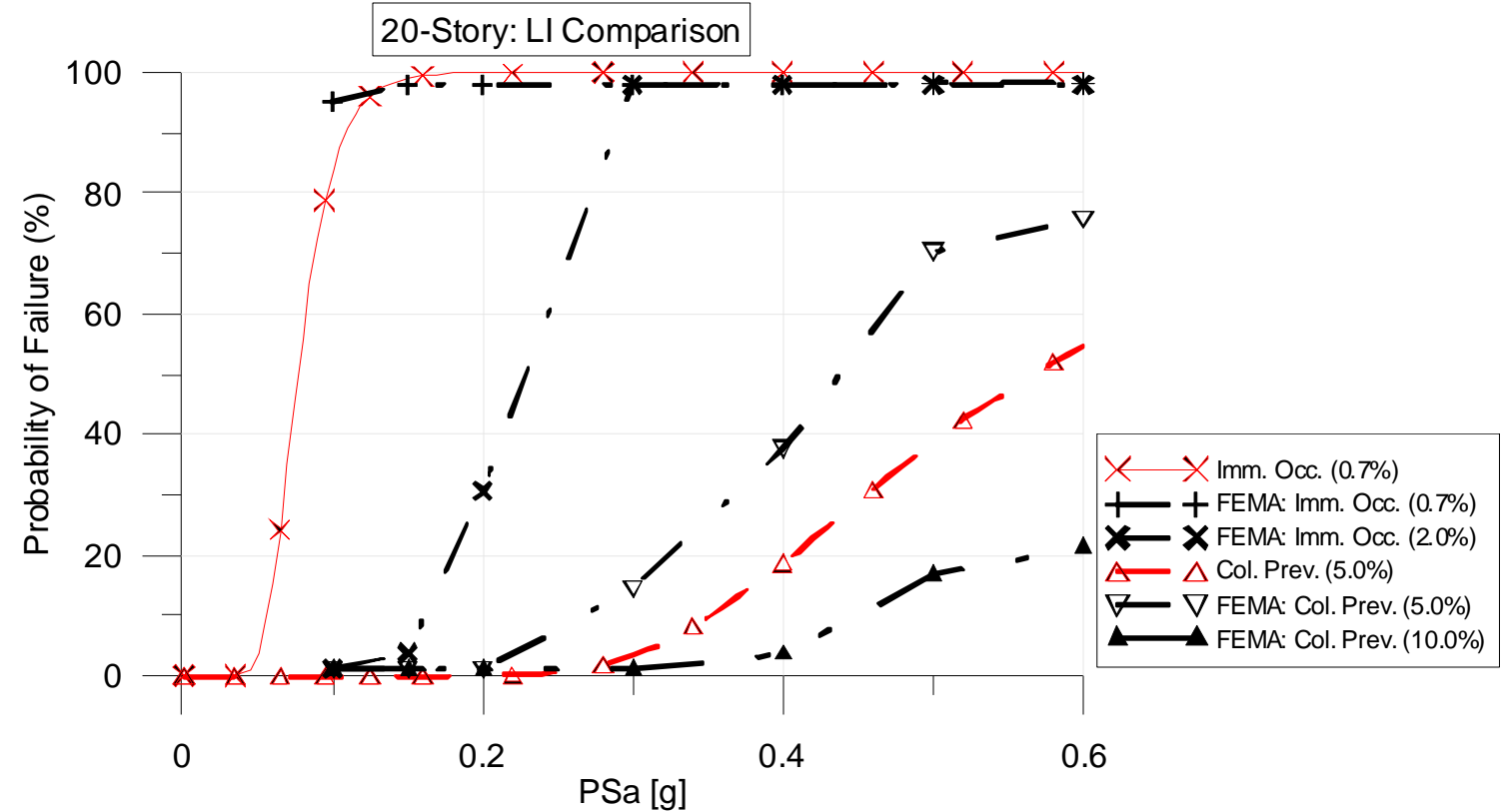

Figure E.61: Regression and FEMA comparison for load intensity, 20-story 


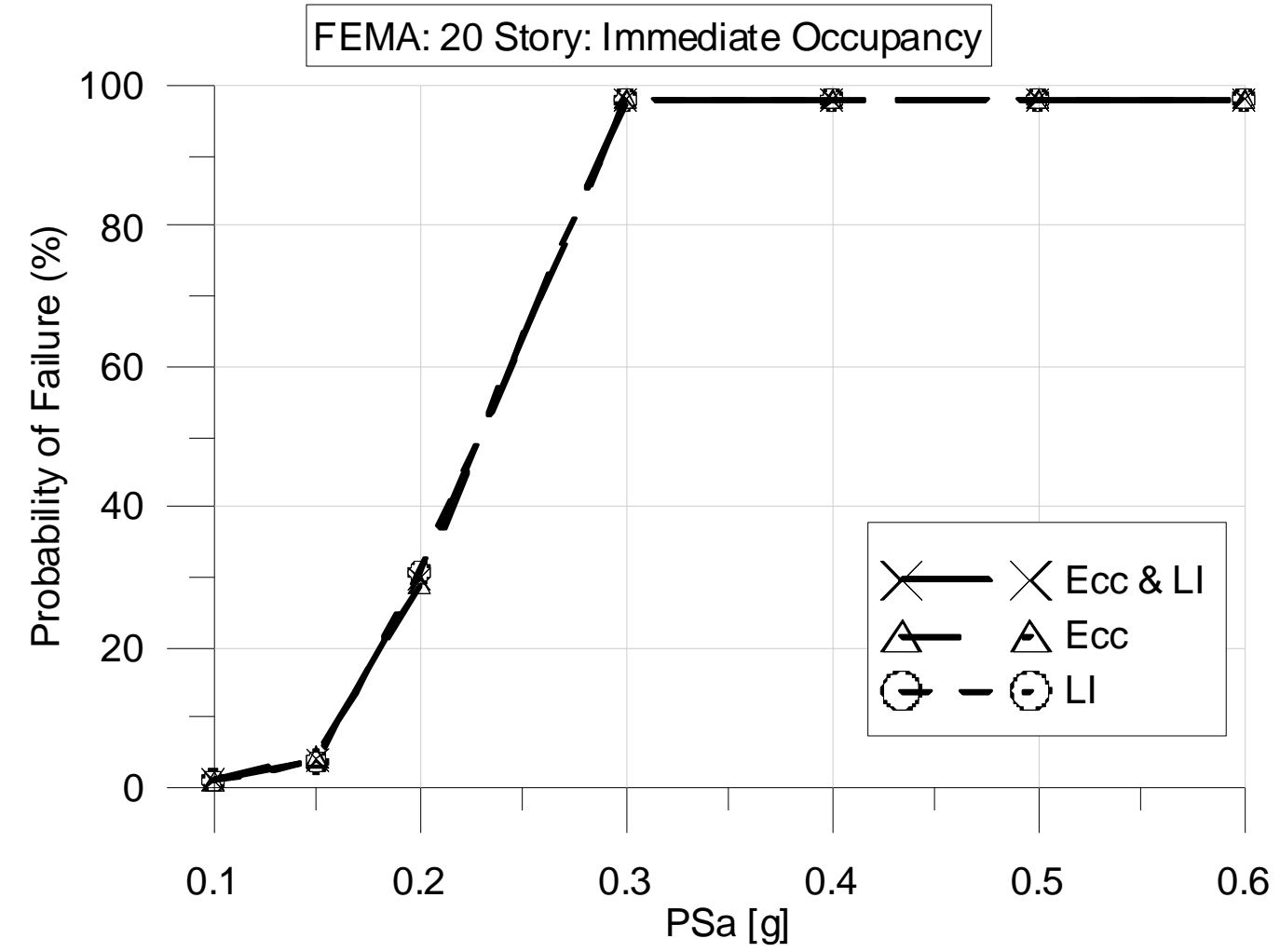

Figure E.62: FEMA 350 fragility curves for immediate occupancy comparison, 20-story

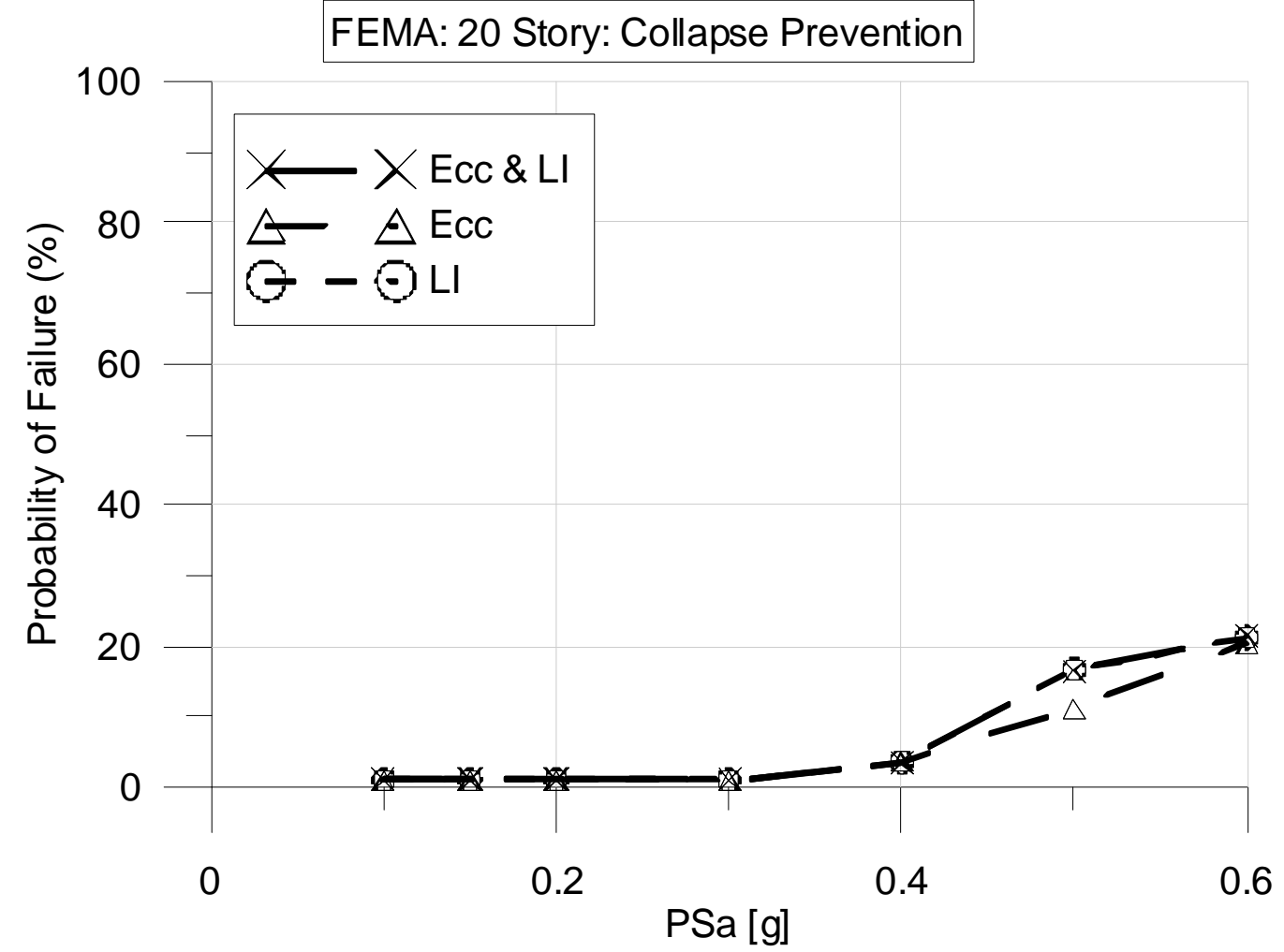

Figure E.63: FEMA 350 fragility curves for collapse prevention comparison, 20-story 